\title{
QUASI-REFLECTION ALGEBRAS AND CYCLOTOMIC ASSOCIATORS
}

\author{
BENJAMIN ENRIQUEZ
}

\begin{abstract}
We develop a cyclotomic analogue of the theory of associators. Using a trigonometric version of the universal KZ equations, we prove the formality of a morphism $B_{n}^{1} \rightarrow$ $(\mathbb{Z} / N \mathbb{Z})^{n} \rtimes \mathfrak{S}_{n}$, where $B_{n}^{1}$ is a braid group of type $B$. The formality isomorphism depends algebraically on a series $\Psi_{\mathrm{KZ}}$, the "KZ pseudotwist". We study the scheme of pseudotwists and show that it is a torsor under a group $\operatorname{GTM}(N, \mathbf{k})$, mapping to Drinfeld's group $\operatorname{GT}(\mathbf{k})$, and whose Lie algebra is isomorphic to its associated graded $\operatorname{grtm}(N, \mathbf{k})$. We prove that Ihara's subgroup GTK of the Grothendieck-Teichmüller group, defined using distribution relations, in fact coincides with it. We show that the subscheme of pseudotwists satisfying distribution relations is a subtorsor. We study the corresponding analogue $\mathfrak{g r t m o}(N, \mathbf{k})$ of $\mathfrak{g r t m}(N, \mathbf{k})$; it is a graded Lie algebra with an action of $(\mathbb{Z} / N \mathbb{Z})^{\times}$, and we give a lower bound for the character of its space of generators.
\end{abstract}

\section{INTRODUCTION}

0.1. The purpose of this paper is to develop a cyclotomic analogue of Drinfeld's theory of associators. This theory can be described as follows. In [Ko], Kohno proves that the pure braid group of order $n$ is formal, i.e., its Malcev Lie algebra is isomorphic to its associated graded Lie algebra, which has an explicit presentation; this result relies on the universal KnizhnikZamolodchikov (KZ) equations, but it can also be proved in the context of minimal model theory $([\mathrm{Su}])$. Kohno's isomorphisms involve the periods of $\mathbb{P}^{1}-\{0,1, \infty\}$ and are therefore defined over $\mathbb{C}$. One of the consequences of [Dr2] is that these isomorphisms are defined over $\mathbb{Q}$. To prove this, Drinfeld shows that the isomorphisms algebraically depend on a particular element in a prounipotent group $F_{2}(\mathbb{C})$, the $\mathrm{KZ}$ associator $\Phi_{\mathrm{KZ}}$. He then defines the scheme of associators over a field $\mathbf{k}$ with $\operatorname{char}(\mathbf{k})=0$ as the set of elements of $\mathbf{k} \times F_{2}(\mathbf{k})$ sharing some properties of ${ }^{1}\left(2 \pi \mathrm{i}, \Phi_{\mathrm{KZ}}\right)$ and proves that a rational associator exists. The main point of this proof is the introduction of a group $\mathrm{GT}(\mathbf{k})$, over which the scheme of associators is a torsor. Its definition relies on the categorical meaning of associators: associators allow to construct quasitriangular quasibialgebras (QTQBA's) and therefore $\mathbf{k}[[\hbar]]$-linear braided monoidal categories, in which the image of the pure braid groups are close to $1 ; \mathrm{GT}(\mathbf{k})$ may be viewed as the group of automorphisms of such structures. This group admits profinite and pro- $l$ variants $\widehat{\mathrm{GT}}$ and $\mathrm{GT}_{l}$, where these categories are replaced by braided monoidal categories, in which the image of the pure braid groups are finite or $l$-groups. These variants play a role in arithmetics; GT(k) may be viewed as an explicit approximation of the motivic fundamental group of $\mathbb{P}^{1}-\{0,1, \infty\}$.

0.2. In this paper, we use universal versions of the "cyclotomic" KZ system introduced in [EE] (see also [GL] for $N=2$ ) to prove (Section 1) the formality of the morphism $B_{n}^{1} \rightarrow$ $(\mathbb{Z} / N \mathbb{Z})^{n} \rtimes \mathfrak{S}_{n}\left(n, N \geq 1 ; B_{n}^{1}\right.$ is a braid group of type $\left.B\right)$. We show (Section 2$)$ that the formality isomorphisms algebraically depend on a pair $\left(\Phi_{\mathrm{KZ}}, \Psi_{\mathrm{KZ}}\right)$, where $\Psi_{\mathrm{KZ}}$ is a generating series for periods of $\mathbb{C}^{\times}-\mu_{N}(\mathbb{C})$. We then introduce a scheme $\underline{\text { Pseudo }}(N, \mathbf{k})$ of all quadruples $(a, \lambda, \Phi, \Psi) \in \mathbb{Z} / N \mathbb{Z} \times \mathbf{k} \times F_{2}(\mathbf{k}) \times F_{N+1}(\mathbf{k})$ sharing some properties of $\left(-\overline{1}, 2 \pi \mathrm{i}, \Phi_{\mathrm{KZ}}, \Psi_{\mathrm{KZ}}\right)$; these are called pseudotwists. A pseudotwist may be viewed as a universal object allowing to

\footnotetext{
${ }^{1}$ We set $\mathrm{i}:=\sqrt{-1}$.
} 
construct quasi-reflection algebras over quasitriangular quasi-bialgebras (Sections 3, 4), and the elements of the preimage $\operatorname{Pseudo}(N, \mathbf{k})$ of $(\mathbb{Z} / N \mathbb{Z})^{\times} \times \mathbf{k}^{\times}$by $\underline{\operatorname{Pseudo}}(N, \mathbf{k}) \rightarrow(\mathbb{Z} / N \mathbb{Z}) \times \mathbf{k}$ give rise to formality isomorphisms for $B_{n}^{1} \rightarrow(\mathbb{Z} / N \mathbb{Z})^{n} \rtimes \mathfrak{S}_{n}$. To show that a $\operatorname{Pseudo}(N, \mathbb{Q})$ is nonempty, we show (Section 7$)$ that $\operatorname{Pseudo}(N, \mathbf{k})$ is a torsor over a group $\operatorname{GTM}(N, \mathbf{k})$, which can be defined as the group of automorphisms of braided module categories over $\mathbf{k}[[\hbar]]$-linear braided monoidal categories, in which the image of $K_{n, N}:=\operatorname{Ker}\left(B_{n}^{1} \rightarrow(\mathbb{Z} / N \mathbb{Z})^{n} \rtimes \mathfrak{S}_{n}\right)$ is close to 1 (Section 5); this group is defined by imposing the defining conditions of a module version GTM of the group GT in products of partially completed groups. The group $\operatorname{GTM}(N, \mathbf{k})$ admits a pro- $l$ variant $\operatorname{GTM}(N)_{l}$ of automorphisms of braided module categories over braided monoidal categories, in which the image of $K_{n, N}$ is a l-group. We have natural morphisms $\pi_{N N^{\prime}}: \operatorname{GTM}(N)_{l} \rightarrow \operatorname{GTM}\left(N^{\prime}\right)_{l}$ for $N^{\prime} \mid N$ (this is an isomorphism if $N^{\prime}=N / l^{\alpha}$, where $\alpha$ is the $l$-adic valuation of $N)$, and morphisms $\operatorname{GTM}(N)_{l} \rightarrow \operatorname{GTM}\left(N, \mathbb{Q}_{l}\right)$ and $\widehat{\operatorname{GT}} \rightarrow \operatorname{GTM}(N)_{l}$ (Section 6). The map $\operatorname{Gal}(\overline{\mathbb{Q}} / \mathbb{Q}) \rightarrow \mathrm{GT}_{l}$ studied by Ihara [Ih1] factors through a sequence of maps $\operatorname{Gal}(\overline{\mathbb{Q}} / \mathbb{Q}) \hookrightarrow \widehat{\mathrm{GT}} \rightarrow \operatorname{GTM}(N)_{l} \rightarrow \mathrm{GT}_{l}$. One can show that the natural morphism $\operatorname{Gal}(\overline{\mathbb{Q}} / \mathbb{Q}) \rightarrow \operatorname{Out}\left(\pi_{1}^{\text {orb }}\left(\left(\mathbb{G}_{m}-\mu_{N}\right) / D_{N}\right)^{(l)}\right)$ factors through $\operatorname{Gal}(\overline{\mathbb{Q}} / \mathbb{Q}) \rightarrow \operatorname{GTM}(N)_{l}(\operatorname{Section}$ $13)$.

We study the group $\operatorname{GRTM}(N, \mathbf{k})$ of $\operatorname{GTM}(N, \mathbf{k})$-automorphisms of $\operatorname{Pseudo}(N, \mathbf{k})$; it is non-canonically isomorphic to $\operatorname{GTM}(N, \mathbf{k})$ and is equipped with a morphism $\operatorname{GRTM}(N, \mathbf{k}) \rightarrow$ $(\mathbb{Z} / N \mathbb{Z})^{\times} \times \mathbf{k}^{\times}$. The Lie algebra $\mathfrak{g r t m}_{(\overline{1}, 1)}(N, \mathbf{k})$ of the preimage of $(\overline{1}, 1)$ is graded, with a $(\mathbb{Z} / N \mathbb{Z})^{\times}$-action.

We then study distribution relations. In Section 8 (which can be read independently), we prove that the inclusion GTK $\subset \widehat{\mathrm{GT}}$ ([Ih2]) is in fact an equality. We then construct distribution morphisms $\delta_{N N^{\prime}}: \operatorname{GTM}(N)_{l} \rightarrow \operatorname{GTM}\left(N^{\prime}\right)_{l}$, for $N^{\prime} \mid N$. Using $\pi_{N N^{\prime}}$ and $\delta_{N N^{\prime}}$, one then constructs a subgroup $\operatorname{GTMD}(N)_{l} \subset \operatorname{GTM}(N)_{l}$. A consequence of Section 8 is that we have a factorization $\widehat{\mathrm{GT}} \rightarrow \operatorname{GTMD}(N)_{l} \subset \operatorname{GTM}(N)_{l}$. We also construct a field version $\operatorname{GTMD}(N, \mathbf{k}) \subset \operatorname{GTM}(N, \mathbf{k})$.

We then show that the level $N$ version $\Psi_{\mathrm{KZ}}^{N}$ of $\Psi_{\mathrm{KZ}}$ satisfies distribution relations. This means that as in [Go, Rac], the pseudotwists $\Psi_{\mathrm{KZ}}^{N}$ and $\Psi_{\mathrm{KZ}}^{N^{\prime}}$ are related by two transformations for $N^{\prime} \mid N$ related to $\pi_{N N^{\prime}}$ and $\delta_{N N^{\prime}}$; this implies a system of equations satisfied by $\Psi_{\mathrm{KZ}}^{N}$. Adjoining these conditions to the defining equations of $\underline{\mathbf{P s e u d o}}(N, \mathbf{k})$, we obtain a diagram $\mathbf{k}^{\nu} \leftarrow \underline{\operatorname{Psdist}}(N, \mathbf{k}) \hookrightarrow \underline{\operatorname{Pseudo}}(N, \mathbf{k})$ (here $\nu$ is the number of distinct prime factors of $N$ ). We show that $\operatorname{Psdist}(N, \mathbf{k}):=\underline{\operatorname{Psdist}}(N, \mathbf{k}) \cap \operatorname{Pseudo}(N, \mathbf{k})$ is a torsor under $\operatorname{GTMD}(N, \mathbf{k}) \subset$ $\operatorname{GTM}(N, \mathbf{k})$ and $\operatorname{GRTMD}(N, \mathbf{k}) \subset \operatorname{GRTM}(N, \mathbf{k})$. We denote the Lie algebra of the kernel of the composed morphism $\operatorname{GRTMD}(N, \mathbf{k}) \subset \operatorname{GRTM}(N, \mathbf{k}) \rightarrow(\mathbb{Z} / N \mathbb{Z})^{\times} \times \mathbf{k}^{\times}$by $\mathfrak{g r t m}_{(\overline{1}, 1)}(N, \mathbf{k})$. This is a $\mathbb{Z}_{>0}$-graded Lie algebra, equipped with an action of $(\mathbb{Z} / N \mathbb{Z})^{\times}$and a morphism of graded Lie algebras $\mathfrak{g r t m}_{(\overline{1}, 1)}(N, \mathbf{k}) \rightarrow \mathfrak{g r t}_{1}(\mathbf{k})$. We use Drinfeld's method ([Dr2], Proposition $6.3)$ to construct elements of $\mathfrak{g r t m} \mathfrak{d}_{(\overline{1}, 1)}(N, \mathbb{C})$; using the nonvanishing of Dirichlet $L$-functions, and the proof of $[\mathrm{W}]$ of the theorem of Bass on cyclotomic units, we derive lower bounds for the character (as a $(\mathbb{Z} / N \mathbb{Z})^{\times}$-module) of the space of generators $\mathfrak{g} /[\mathfrak{g}, \mathfrak{g}]$ of $\mathfrak{g}:=\mathfrak{g r t m} \mathfrak{d}_{(\overline{1}, 1)}(N, \mathbb{C})$ (Section 11). We derive from this the surjectivity of a group morphism $\operatorname{GRTMD}(N, \mathbf{k}) \rightarrow$ $(\mathbb{Z} / N \mathbb{Z})^{\times} \times\left(\mathbf{k}^{\nu} \rtimes \mathbf{k}^{\times}\right)$and therefore of the map $\operatorname{Psdist}(N, \mathbf{k}) \rightarrow(\mathbb{Z} / N \mathbb{Z})^{\times} \times\left(\mathbf{k}^{\nu} \rtimes \mathbf{k}^{\times}\right)$(Section 10.6).

Finally, we relate the Lie algebras $\mathfrak{g r t m}_{(\overline{1}, 1)}(N, \mathbf{k})$ and $\mathfrak{g r t m} \mathfrak{d}_{(\overline{1}, 1)}(N, \mathbf{k})$ with a generalization of Ihara's algebra, defined using special derivations (Section 12).

0.3. This paper is related to the following works:

(a) in [Rac], Racinet introduces a torsor $\operatorname{DMRD}(N, \mathbf{k})$ over a prounipotent Lie group, based on the shuffle and distribution relations satisfied by special values of multiple polylogarithms (which coincide with the periods involved in $\Psi_{\mathrm{KZ}}$, see Appendix A). He conjectures that for 
$N=1$, there is a morphism of torsors $\operatorname{DMRD}(1, \mathbf{k}) \rightarrow \underline{\operatorname{Assoc}}(\mathbf{k})$ taking the analogue of $\Phi_{\mathrm{KZ}}$ in $\operatorname{DMRD}(1, \mathbb{C})$ to $\Phi_{\mathrm{KZ}}$, and that this is an isomorphism. A natural generalization of this conjecture would be to expect a morphism of torsors $\operatorname{DMRD}(N, \mathbf{k}) \rightarrow \underline{\operatorname{Psdist}}(N, \mathbf{k})$ when $N \neq 1$.

(b) in [DG], Section 5, Deligne and Goncharov studied the motivic fundamental group of $\mathbb{G}_{m}-\mu_{N}$, of which $\operatorname{GTMD}(N, \mathbf{k})$ should be an explicit approximation. The element $\Psi_{\mathrm{KZ}}$ appears in [DG], Proposition 5.17 and "motivic" conditions satisfied by $\Psi_{\mathrm{KZ}}$ are given in Section 5.19. The lower bound found in Section 11 for the character of the space of generators of $\mathfrak{g r t m} \mathfrak{d}_{(\overline{1}, 1)}(N, \mathbb{C})$ seems related to the upper bound of [DG], Cor. 5.25 for the dimension of the $\mathbb{Q}$-linear span of the coefficients of $\Psi_{\mathrm{KZ}}$ of fixed degree.

(c) in $[\mathrm{Ih} 1]$, Ihara studies the kernel of $\operatorname{Gal}\left(\overline{\mathbb{Q}} / \mathbb{Q}\left(\mu_{l^{\infty}}\right)\right) \rightarrow \mathrm{GT}_{l}$; this leads to a graded Lie algebra over $\mathbb{Z}_{l}$, in which he constructed analogues of the Drinfeld generators (using Soulé's cyclotomic characters) and conjectural integral versions of these generators. These constructions should generalize to the morphism $\operatorname{Gal}\left(\overline{\mathbb{Q}} / \mathbb{Q}\left(\mu_{N l^{\infty}}\right)\right) \rightarrow \operatorname{GTMD}(N)_{l}$.

(d) using twists of QRA's over QTQBA's, one can hope for a generalization of the KohnoDrinfeld theorem to representations of $B_{n}^{1}$ arising from specialized versions of the cyclotomic KZ equations. This study was started in [GL] for $N=2$.

Acknowledgements. This project was started in collaboration with P. Etingof in September 2003. I wish to thank him heartily for collaboration in [EE] and for many discussions at several stages of this work. I also would like to thank D. Calaque, F. Lecomte, V. Leksin, I. Marin and V. Toledano Laredo for comments.

\section{The Formality of $B_{n}^{1} \rightarrow(\mathbb{Z} / N \mathbb{Z})^{n} \rtimes \mathfrak{S}_{n}$}

We fix a base field $\mathbf{k}$ of characteristic 0 . If $\Gamma$ is a finitely generated group, we denote by $\Gamma(\mathbf{k})$ the corresponding Malcev pro-unipotent group and by $\operatorname{Lie}(\Gamma(\mathbf{k}))$ the Lie algebra of $\Gamma(\mathbf{k})$; if $\mathbf{k} / \mathbb{Q}$ is a finite field extension, then $\operatorname{Lie}(\Gamma(\mathbf{k}))=\operatorname{Lie}(\Gamma(\mathbb{Q})) \otimes \mathbf{k}$.

1.1. Partial prounipotent completions. Let $\varphi: \Gamma \rightarrow \Gamma_{0}$ be a surjective group morphism. Assume that $\Gamma_{1}:=\operatorname{Ker} \varphi$ is finitely generated. Then one can construct a non-connected proalgebraic group $\Gamma(\varphi, \mathbf{k})$, fitting in an exact sequence $1 \rightarrow \Gamma_{1}(\mathbf{k}) \rightarrow \Gamma(\varphi, \mathbf{k}) \rightarrow \Gamma_{0} \rightarrow 1$, and a group morphism $\Gamma \rightarrow \Gamma(\varphi, \mathbf{k})$, such that the diagram

$$
\begin{aligned}
& 1 \rightarrow \Gamma_{1} \quad \rightarrow \quad \Gamma \quad \rightarrow \quad \Gamma_{0} \rightarrow 1
\end{aligned}
$$

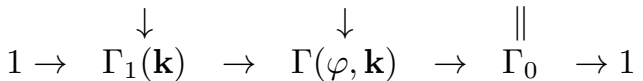

commutes, and with the following properties.

$$
\Gamma \rightarrow \Gamma(\varphi, \mathbf{k})
$$

The commuting triangle $\searrow \quad \swarrow \quad$ is an initial object in the category where objects are $\Gamma_{0}$

similar commuting triangles, where $\Gamma(\varphi, \mathbf{k})$ is replaced by a non-connected prounipotent group $U$, and morphisms are morphisms $U \rightarrow U^{\prime}$, such that the resulting tetrahedron commutes. The group $\Gamma(\varphi, \mathbf{k})$ is then called the partial prounipotent completion of $\Gamma \stackrel{\varphi}{\rightarrow} \Gamma_{0}$. A commuting

$$
\begin{array}{rccccc}
\Gamma & \rightarrow & \Gamma_{0} & \Gamma(\varphi, \mathbf{k}) & \rightarrow & \Gamma_{0} \\
\text { square } & \downarrow & & \downarrow \text { gives rise to a commuting square } \begin{array}{r}
\downarrow \\
\Gamma^{\prime}
\end{array} & & \downarrow \\
\Gamma^{\prime} & \stackrel{\varphi^{\prime}}{\rightarrow} & \Gamma_{0}^{\prime} & \Gamma^{\prime}\left(\varphi^{\prime}, \mathbf{k}\right) & \rightarrow & \Gamma_{0}^{\prime}
\end{array}
$$

1.2. Formality of a group morphism. Recall that the finitely generated group $\Gamma$ is called formal iff there exists a Lie algebra isomorphism $\operatorname{Lie}(\Gamma(\mathbf{k})) \rightarrow$ gr $\operatorname{Lie}(\Gamma(\mathbf{k}))$, whose associated graded morphism is the identity (here $\operatorname{gr}(\mathfrak{g})$ is the graded Lie algebra associated to a pronilpotent Lie algebra $\mathfrak{g}$ and $\hat{\operatorname{gr}}(\mathfrak{g})$ is the degree completion of $\operatorname{gr}(\mathfrak{g}))$. 
In the above situation, the choice of a section $\gamma_{0} \mapsto \tilde{\gamma}_{0}$ of $\Gamma(\mathbf{k}) \rightarrow \Gamma_{0}$ induces a map $\Gamma_{0} \rightarrow$ $\operatorname{Aut}\left(\operatorname{Lie}\left(\Gamma_{1}(\mathbf{k})\right)\right), \gamma_{0} \mapsto \theta_{\gamma_{0}}$, where $\theta_{\gamma_{0}}(x)=\tilde{\gamma}_{0} x \tilde{\gamma}_{0}^{-1}$; the map $\gamma_{0} \mapsto \theta_{\gamma_{0}}$ may fail to be a group morphism (by inner automorphisms). Each $\theta_{\gamma_{0}}$ induces a graded Lie algebra automorphism $\operatorname{gr} \theta_{\gamma_{0}} \in \operatorname{Aut}\left(\operatorname{gr} \operatorname{Lie}\left(\Gamma_{1}(\mathbf{k})\right)\right)$, which is independent of the choice of the lifting $\gamma_{0} \mapsto \tilde{\gamma}_{0}$, and $\gamma_{0} \mapsto$ $\operatorname{gr} \theta_{\gamma_{0}}$ is a group morphism. We can then form the semidirect product $\exp \left(\hat{\mathrm{gr}} \operatorname{Lie} \Gamma_{1}(\mathbf{k})\right) \rtimes \Gamma_{0}$.

We say that the group morphism $\varphi: \Gamma \rightarrow \Gamma_{0}$ is formal if there exists a group isomorphism $\Gamma(\mathbf{k}, \varphi) \simeq \exp \left(\operatorname{gr} \operatorname{Lie} \Gamma_{1}(\mathbf{k})\right) \rtimes \Gamma_{0}$, restricting to a formality isomorphism for $\Gamma_{1}$, and such that the diagram

$$
\begin{aligned}
& 1 \rightarrow \quad \Gamma_{1}(\mathbf{k}) \quad \rightarrow \quad \Gamma(\mathbf{k}, \varphi) \quad \rightarrow \quad \Gamma_{0} \rightarrow 1
\end{aligned}
$$

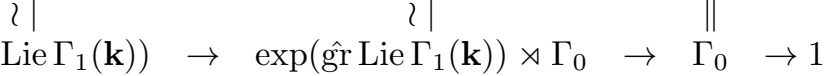

commutes.

For example, the morphism $B_{n} \rightarrow \mathfrak{S}_{n}$ is formal, where $B_{n}$ is the braid group of order $n$ (when $\mathbf{k}=\mathbb{C}$, this follows from $[\mathrm{Ko}]$, and for $\mathbf{k}=\mathbb{Q}$, from $[\mathrm{Dr} 2]$ ).

1.3. The morphism $\varphi_{n, N}: B_{n}^{1} \rightarrow(\mathbb{Z} / N \mathbb{Z})^{n} \rtimes S_{n}$. Let $B_{n}^{1}:=B_{n+1} \times_{\mathfrak{S}_{n+1}} \mathfrak{S}_{n}$, where $\mathfrak{S}_{n} \subset \mathfrak{S}_{n+1}$ is the subgroup of permutations of $[0, N]$ such that $\sigma(0)=0$. We have $B_{n}^{1}=$ $\pi_{1}\left(X_{n}, \mathfrak{S}_{n} b\right)$, where $X_{n}:=\left[\left(\mathbb{C}^{\times}\right)^{n}-\cup_{1<i<j<n} D_{i j}\right] / \mathfrak{S}_{n}, D_{i j}=\left\{\left(Z_{1}, \ldots, Z_{n}\right) \mid Z_{i}=Z_{j}\right\}$ and $b:=\left\{\left(Z_{1}, \ldots, Z_{n}\right) \mid 0<Z_{1}<\ldots<Z_{n}\right\}$.

Let $W_{n, N}:=\left(\mathbb{C}^{\times}\right)^{n}-\cup_{1 \leq i<j \leq n} \cup_{\zeta \in \mu_{N}(\mathbb{C})} D_{i j, \zeta}$, where $D_{i j, \zeta}=\left\{\left(z_{1}, \ldots, z_{n}\right) \mid z_{i}=\zeta z_{j}\right\}$. Then the map $W_{n, N} \rightarrow X_{n}$ induced by $\left(z_{1}, \ldots, z_{n}\right) \rightarrow\left[\left(z_{1}^{N}, \ldots, z_{n}^{N}\right)\right]$ is a covering with group $(\mathbb{Z} / N \mathbb{Z})^{n} \rtimes \mathfrak{S}_{n}$; it induces a group morphism $\varphi_{n, N}: B_{n}^{1} \rightarrow(\mathbb{Z} / N \mathbb{Z})^{n} \rtimes \mathfrak{S}_{n}$. We have then $K_{n, N}:=\operatorname{Ker} \varphi_{n, N} \simeq$ $\pi_{1}\left(W_{n, N}, b\right)$.

We now introduce the universal "cyclotomic" KZ system and use it to prove the formality of $\varphi_{n, N}$.

1.4. The universal cyclotomic $\mathbf{K Z}$ system. Let $\mathfrak{t}_{n, N}(\mathbf{k})\left(\right.$ or $\left.\mathfrak{t}_{n, N}\right)$ be the $\mathbf{k}$-Lie algebra with generators $t_{0}^{0 i},(i \in[1, n])$, and $t(a)^{i j},(i \neq j \in[1, n], a \in \mathbb{Z} / N \mathbb{Z})$, and relations:

$$
\begin{gathered}
t(a)^{i j}=t(-a)^{j i}, \quad\left[t(a)^{i j}, t(a+b)^{i k}+t(b)^{j k}\right]=0, \quad\left[t_{0}^{0 i}+t_{0}^{0 j}+\sum_{b \in \mathbb{Z} / N \mathbb{Z}} t(b)^{i j}, t(a)^{i j}\right]=0, \\
{\left[t_{0}^{0 i}, t_{0}^{0 j}+\sum_{b \in \mathbb{Z} / N \mathbb{Z}} t(b)^{i j}\right]=0, \quad\left[t_{0}^{0 i}, t(a)^{j k}\right]=0, \quad\left[t(a)^{i j}, t(b)^{k l}\right]=0}
\end{gathered}
$$

$(i, j, k, l \in[1, n]$ are all distinct and $a, b \in \mathbb{Z} / N \mathbb{Z})$. The group $(\mathbb{Z} / N \mathbb{Z})^{n} \rtimes \mathfrak{S}_{n}$ acts on $\mathfrak{t}_{n, N}$ as follows: let $s_{i}=(\overline{0}, \ldots, \overline{1}, \ldots, \overline{0})$ be the $i$ th generator of $(\mathbb{Z} / N \mathbb{Z})^{n}$, then $s_{i} \cdot t_{0}^{0 j}=t_{0}^{0 j}$ and $s_{i} \cdot t(a)^{j k}=t\left(a+\delta_{i j}-\delta_{i k}\right)^{j k} ;$ for $\sigma \in \mathfrak{S}_{n}, \sigma \cdot t_{0}^{0 i}=t_{0}^{0 \sigma(i)}, \sigma \cdot t(a)^{i j}=t(a)^{\sigma(i) \sigma(j)}$.

When $\mathbf{k}=\mathbb{C}$, we define $t[\zeta]^{i j}$ for $\zeta \in \mu_{N}(\mathbb{C})$ by $t\left[\zeta_{N}^{a}\right]^{i j}=t(a)^{i j}$, where $\zeta_{N}:=e^{2 \pi \mathrm{i} / N} \in \mu_{N}(\mathbb{C})$. Set

then the connection

$$
K_{i}\left(z_{1}, \ldots, z_{n}\right):=\frac{t_{0}^{0 i}}{z_{i}}+\sum_{j \in[1, n]-\{i\}} \sum_{\zeta \in \mu_{N}(\mathbb{C})} \frac{t[\zeta]^{i j}}{z_{i}-\zeta z_{j}},
$$

$$
\mathrm{d}-\sum_{i=1}^{n} K_{i}\left(z_{1}, \ldots, z_{n}\right) \mathrm{d} z_{i}
$$

on the trivial $\exp \left(\hat{\mathfrak{t}}_{n, N}(\mathbb{C})\right)$-bundle ${ }^{2}$ over $W_{n, N}$ is flat. This connection is also equivariant w.r.t. the action of $\Gamma_{0}:=(\mathbb{Z} / N \mathbb{Z})^{n} \rtimes \mathfrak{S}_{n}$, therefore its descends to a flat connection on the $\exp \left(\hat{\mathfrak{t}}_{n, N}(\mathbb{C})\right) \rtimes \Gamma_{0}$-bundle over $X_{N}$, such that the set of sections of $\mathcal{U} \subset X_{N}$ is the set of

\footnotetext{
$2 \hat{\mathfrak{g}}$ denotes the degree completion of a $\mathbb{N}$-graded Lie algebra; here $\operatorname{deg}\left(t_{0}^{0 i}\right)=\operatorname{deg}\left(t(a)^{i j}\right)=1$.
} 
functions $f: \pi^{-1}(\mathcal{U}) \rightarrow \exp \left(\hat{\mathfrak{t}}_{n, N}(\mathbb{C})\right) \rtimes \Gamma_{0}$, such that $f\left(\gamma_{0} \cdot x\right)=f(x) \gamma_{0}^{-1}$ for $\gamma_{0} \in \Gamma_{0}$ (here $\pi: W_{n, N} \rightarrow X_{n}$ is the canonical projection).

1.5. Monodromy morphisms. There exists a unique solution $F: \tilde{X}_{N} \rightarrow \exp \left(\hat{\mathfrak{t}}_{n, N}(\mathbb{C})\right)$ of the system $\partial F / \partial z_{i}=K_{i} F$, with asymptotic behavior $F\left(z_{1}, \ldots, z_{n}\right) \simeq z_{1}^{t_{0}^{0 i}} z_{2}^{t_{0}^{01,2}} \ldots z_{n}^{t_{0}^{0} \ldots n-1, n}$ when $z_{1} \ll z_{2} \ldots \ll z_{n}$; here $t_{0}^{0 \ldots r-1, r}=t_{0}^{0 r}+\sum_{i=1}^{r-1} \sum_{\zeta \in \mu_{N}(\mathbb{C})} t[\zeta]^{i r}$. Here $\tilde{X}_{N} \rightarrow X_{N}$ is the universal cover map.

If now $\gamma \in B_{n}^{1}$, the map $z \mapsto F(z)^{-1} \varphi_{n, N}(\gamma) F\left(\gamma^{-1} z\right) \in \exp \left(\hat{\mathfrak{t}}_{n, N}(\mathbb{C})\right) \rtimes \Gamma_{0}$ is a constant, called $\mu_{n}(\gamma)$. This defines a group morphism

$$
\mu_{n}: B_{n}^{1} \rightarrow \exp \left(\hat{\mathfrak{t}}_{n, N}(\mathbb{C})\right) \rtimes \Gamma_{0},
$$

which factors through a morphism $B_{n}^{1}\left(\varphi_{n, N}, \mathbb{C}\right) \rightarrow \exp \left(\hat{\mathfrak{t}}_{n, N}(\mathbb{C})\right) \rtimes \Gamma_{0}$. This morphism commutes with the projections to $\Gamma_{0}$, and therefore restricts to a morphism $\mu_{n}(\mathbb{C}): K_{n, N}(\mathbb{C}) \rightarrow$ $\exp \left(\hat{\mathfrak{t}}_{n, N}(\mathbb{C})\right)$.

1.6. Formality of $\varphi_{n, N}$. To prove that $\varphi_{n, N}$ is formal, it then suffices to prove that Lie $\mu_{n}(\mathbb{C})$ : $\operatorname{Lie}\left(K_{n, N}(\mathbb{C})\right) \rightarrow \hat{\mathfrak{t}}_{n, N}(\mathbb{C})$ is an isomorphism.

$K_{n, N}$ may be be viewed as the kernel of the morphism $P_{n+1} \rightarrow(\mathbb{Z} / N \mathbb{Z})^{n}$ given by $x_{i j} \mapsto 0$ if $i, j \neq 0, x_{0 i} \mapsto s_{i}=(\overline{0} \ldots \overline{1} \ldots \overline{0})$ if $i \neq 0$ (here the $x_{i j}, 0 \leq i<j \leq n$ are the Artin generators of the pure braid group $\left.P_{n+1}\right)$. Set $X_{0 i}:=x_{0 i}^{N}, x_{i j}(\alpha):=x_{0 i}^{\alpha} x_{i j} x_{0 i}^{-\alpha}$ for $\alpha \in \mathbb{Z}$.

One can show that the $X_{0 i}$ and $x_{i j}(\alpha)(i \neq j \in[1, n], \alpha \in[0, N-1])$ are generators of $K_{n, N}$. We have: (a) Lie $\mu_{n}(\mathbb{C})$ takes $\left[\log X_{0 i}\right]$ and $\left[\log x_{i j}(\alpha)\right]$ to multiples to $t_{0}^{0 i}$ and $t(\bar{\alpha})^{i j}$ (here $X_{0 i}, x_{i j}(\alpha)$ are identified with their images in $K_{n, N}(\mathbb{C}),[x]$ is the class of $x \in \operatorname{Lie}\left(K_{n, N}(\mathbb{C})\right)$ in $\left.\operatorname{gr}_{1} \operatorname{Lie}\left(K_{n, N}(\mathbb{C})\right)\right)$ and $\alpha \mapsto \bar{\alpha}$ is the canonical projection $\mathbb{Z} / \rightarrow \mathbb{Z} / N \mathbb{Z}$; (b) one can exhibit relations between these generators, whose logarithms have degree $\geq 2$, and whose degree 2 parts coincide with the defining relations of $\mathfrak{t}_{n, N}(\mathbb{C})$. These relations are explicitly given as follows (recall that $x_{i j}(\alpha+N)=X_{0 i} x_{i j}(\alpha) X_{0 i}^{-1}$, so $\left[\log x_{i j}(\alpha)\right]$ depends only on $\left.\bar{\alpha}\right)$ :

Proposition 1.1.

$$
\begin{gathered}
\left(X_{0 j} x_{i j}(0) \ldots x_{i j}(N-1) X_{0 i}, X_{0 j}\right)=\left(X_{0 j} x_{i j}(0) \ldots x_{i j}(N-1) X_{0 i}, x_{i j}(\alpha)\right)=1, \\
\left(x_{i j}(\alpha) x_{i k}(\alpha+\beta \mid \alpha) u_{i k}(\alpha, \beta) x_{j k}(\beta \mid 0) u_{i k}(\alpha, \beta)^{-1}, x_{i j}(\alpha)\right) \\
=\left(x_{i j}(\alpha) x_{i k}(\alpha+\beta \mid \alpha) u_{i k}(\alpha, \beta) x_{j k}(\beta \mid 0) u_{i k}(\alpha, \beta)^{-1}, x_{i k}(\alpha+\beta \mid \alpha)\right)=1, \\
\left(x_{i j}(\alpha), u_{i k}(\alpha,-\beta) x_{k l}(\beta) u_{i k}(\alpha,-\beta)^{-1}\right)=\left(u_{i j}(\alpha+1,-\beta)^{-1} x_{i l}(\alpha) u_{i j}(\alpha+1,-\beta), x_{j k}(\beta)\right) \\
=\left(\operatorname{Ad}\left[u_{i j}(\alpha+1,-\beta-1)^{-1}\right]\left[x_{i k}(\alpha)\right], \operatorname{Ad}\left[u_{i j}(\alpha,-\beta-1)^{-1} u_{i j}(\alpha,-\beta)\right]\left[x_{j l}(\beta)\right]\right)=1, \\
\left(u_{i j}(N,-\alpha)^{-1} X_{0 i}, x_{j k}(\alpha)\right)=\left(u_{i k}(\alpha,-N) X_{0 k}, x_{i j}(\alpha)\right)=\left(u_{i j}(\alpha+1,-N) X_{0 j}, x_{i k}(\alpha)\right)=1
\end{gathered}
$$

where $i<j<k<l, \alpha, \beta \in \mathbb{Z}, x_{i k}(\alpha+\beta \mid \alpha):=\operatorname{Ad}\left[x_{i k}(\alpha) x_{i k}(\alpha+1) \ldots x_{i k}(\alpha+\beta-1)\right]\left(x_{i k}(\alpha+\beta)\right)$ and $u_{i k}(\alpha, \beta)=x_{i k}(\alpha) \ldots x_{i k}(\alpha+\beta-1) x_{i k}(\beta-1)^{-1} \ldots x_{i k}(0)^{-1}$; when $\beta \leq 0, x_{i k}(\alpha) x_{i k}(\alpha+$ 1)... $x_{i k}(\alpha+\beta-1)$ means $x_{i k}(\alpha-1)^{-1} x_{i k}(\alpha-2)^{-1} \ldots x_{i k}(\alpha+\beta)^{-1}$ (this is 1 if $\left.\beta=0\right)$.

Proof. These relations are consequences of the pure braid group relations $\left(x_{i j} x_{i k} x_{j k}, x_{i j}\right)=$ $\left(x_{i j} x_{i k} x_{j k}, x_{j k}\right)=1$ for $i<j<k,\left(x_{i j}, x_{k l}\right)=\left(x_{i l}, x_{j k}\right)=\left(x_{i k}, x_{i j}^{-1} x_{j l} x_{i j}\right)=1$ for $i<j<k<l$ ([Art] $]$; for example, (1) is the conjugation of $\left(x_{i j} x_{i k} x_{j k}, x_{i j}\right)=\left(x_{i j} x_{i k} x_{j k}, x_{i k}\right)=1$ by $x_{0 i}^{\alpha} x_{0 k}^{-\beta}$, using the relations $\operatorname{Ad}\left(x_{0 i}^{\alpha} x_{0 k}^{-\beta}\right)\left(x_{i k}\right)=x_{i k}(\alpha+\beta \mid \beta)$ and $\left(x_{0 i}^{\alpha}, x_{0 k}^{-\beta}\right)=u_{i k}(\alpha, b) ;(2)$ is the conjugation of $\left(x_{i j}, x_{j k}\right)=1$ by $x_{0 i}^{\alpha} x_{0 j}^{\beta}$, of $\left(x_{i l}, x_{j k}\right)=1$ by $x_{0 j}^{\beta} x_{0 i}^{\alpha}$ and of $\left(x_{i k}, x_{j l}(-1)\right)=1$ by $x_{0 j}^{\beta+1} x_{0 i}^{\alpha}$; 
for example, in this last case, we have $\operatorname{Ad}\left[x_{0 j}^{\beta+1} x_{0 i}^{\alpha}\right]\left[x_{i k}\right]=\operatorname{Ad}\left[\left(x_{0 j}^{\beta+1}, x_{0 i}^{\alpha+1}\right) x_{0 i}^{\alpha+1} x_{0 j}^{\beta+1}\right]\left[x_{i k}(-1)\right]=$ $\operatorname{Ad}\left[u_{i j}(\alpha+1,-\beta-1)^{-1}\right]\left[x_{i k}(\alpha)\right]$ and $\operatorname{Ad}\left[x_{0 j}^{\beta+1} x_{0 i}^{\alpha}\right]\left[x_{j l}(-1)\right]=\operatorname{Ad}\left[\left(x_{0 j}^{\beta+1}, x_{0 i}^{\alpha}\right)\left(x_{0 i}^{\alpha}, x_{0 j}^{\beta}\right) x_{0 j}^{\beta} x_{0 i}^{\alpha}\right]\left[x_{j l}\right]=$ $\operatorname{Ad}\left[u_{i j}(\alpha,-\beta-1)^{-1} u_{i j}(\alpha,-\beta)\right]\left[x_{j l}(\beta)\right] ;(3)$ is the conjugation of $\left(x_{0 i}^{N}, x_{j k}\right)=1$ by $x_{0 j}^{\alpha}$, of $\left(x_{0 k}^{N}, x_{i j}\right)=1$ by $x_{0 i}^{\alpha}$, and of $\left(x_{0 j}^{N}, x_{i k}(-1)\right)=1$ by $x_{0 i}^{\alpha+1}$.

It then follows from (b) that we have a morphism $\alpha_{n}: \mathfrak{t}_{n, N}(\mathbb{C}) \rightarrow \operatorname{gr} \operatorname{Lie}\left(K_{n, N}(\mathbb{C})\right)$ given by $t_{0}^{0 i} \mapsto\left[\log X_{0 i}\right], t(a)^{i j} \mapsto\left[\log x_{i j}(\tilde{a})\right]$ ( $\tilde{a}$ is the lift of $a$ to $[0, N-1]$ ). This morphism is surjective, since gr $\operatorname{Lie}\left(K_{n, N}(\mathbb{C})\right)$ is generated in degree 1 , as the associated graded Lie algebra of the quotient of a free Lie algebra (recall that Lie $K_{n, N}(\mathbb{C})$ is the quotient of the topologically free Lie algebra generated by the $\log X_{0 i}, \log x_{i j}(\alpha)$ by the logarithm of their relations). It follows from (a) that gr $\mu_{n}(\mathbb{C}): \operatorname{gr} \operatorname{Lie}\left(K_{n, N}(\mathbb{C})\right) \rightarrow \mathfrak{t}_{n, N}(\mathbb{C})$ is surjective, and one checks that $\operatorname{gr} \mu_{n}(\mathbb{C}) \circ \alpha_{n}$ is bijective. So $\alpha_{n}$ is injective, hence an isomorphism, hence so it gr $\mu_{n}(\mathbb{C})$. So $\mu_{n}(\mathbb{C})$ is an isomorphism, as wanted.

\section{Algebraic Construction of formality isomorphisms}

\subsection{Insertion-coproduct morphisms and structure of the $\mathfrak{t}_{n, N}$.}

2.1.1. Let $\mathfrak{t}_{n}(\mathbf{k})$ ( or $\mathfrak{t}_{n}$ ) be the $\mathbf{k}$-Lie algebra of infinitesimal pure braids introduced in [Dr2]; it has generators $t^{i j}, i \neq j \in[1, n]$, and relations $t^{i j}=t^{j i},\left[t^{i j}, t^{i k}+t^{j k}\right]=0$ and $\left[t^{i j}, t^{k l}\right]=0$ for $i, j, k, l$ distinct.

Let $f:[0, m] \rightarrow[0, n]$ be a partially defined function such that $f(0)=0$, then there is a unique Lie algebra morphism $\mathfrak{t}_{n, N} \rightarrow \mathfrak{t}_{m, N}, x \mapsto x^{f}$, such that

$$
\begin{gathered}
\left(t(a)^{i j}\right)^{f}=\sum_{i^{\prime} \in f^{-1}(i), j^{\prime} \in f^{-1}(j)} t(a)^{i^{\prime} j^{\prime}} \quad(i \neq j \in[1, n]) \\
\left(t_{0}^{0 j}\right)^{f}=\sum_{j^{\prime} \in f^{-1}(j)} t_{0}^{0 j^{\prime}}+\sum_{j^{\prime}, j^{\prime \prime} \in f^{-1}(j), j^{\prime}<j^{\prime \prime}} \sum_{b \in \mathbb{Z} / N \mathbb{Z}} t(b)^{j^{\prime} j^{\prime \prime}}+\sum_{i^{\prime} \in f^{-1}(0) \backslash\{0\}, j^{\prime} \in f^{-1}(j)} \sum_{b \in \mathbb{Z} / N \mathbb{Z}} t(b)^{i^{\prime} j^{\prime}} \quad(j \neq 0) .
\end{gathered}
$$

Let $f:[1, m] \rightarrow[1, n]$ be a partially defined function. There is a unique Lie algebra morphism $\mathfrak{t}_{n} \rightarrow \mathfrak{t}_{m, N}, x \mapsto x^{f}$, such that

$$
\left(t^{i j}\right)^{f}=\sum_{i^{\prime} \in f^{-1}(i), j^{\prime} \in f^{-1}(j)} t(0)^{i^{\prime} j^{\prime}} \quad(i \neq j \in[1, n]) .
$$

We denote $x^{f}$ as $x^{f^{-1}(0), \ldots, f^{-1}(n)}\left(x^{f^{-1}(1), \ldots, f^{-1}(n)}\right.$ in the second case).

We also denote by $x \mapsto x^{f}$ the induced morphisms $U\left(\mathfrak{t}_{n, N}\right) \rightarrow U\left(\mathfrak{t}_{m, N}\right), U\left(\mathfrak{t}_{n}\right) \rightarrow U\left(\mathfrak{t}_{m, N}\right)$.

We denote by $T_{n, N}$ the semidirect product $U\left(\mathfrak{t}_{n, N}\right) \rtimes(\mathbb{Z} / N \mathbb{Z})^{n}$ and by $s_{i} \in T_{n, N}$ the image of the $i$ th generator of $(\mathbb{Z} / N \mathbb{Z})^{n}$.

If $f:[0, m] \rightarrow[0, n]$ is a partially defined function with $f(0)=0$, then the algebra morphism $U\left(\mathfrak{t}_{n, N}\right) \rightarrow U\left(\mathfrak{t}_{m, N}\right), x \mapsto x^{f}$ extends to an algebra morphism $T_{n, N} \rightarrow T_{m, N}$ by $s_{i} \mapsto \prod_{i^{\prime} \in f^{-1}(i)} s_{i^{\prime}}$.

2.1.2. Let us describe the structure of $\mathfrak{t}_{n, N}$. Let $\mathfrak{f}_{n, N} \subset \mathfrak{t}_{n, N}$ be its Lie subalgebra generated by $t_{0}^{0 n}$ and the $t(a)^{i n}(i \in[1, n-1], a \in \mathbb{Z} / N \mathbb{Z})$. These generators freely generate $\mathfrak{f}_{n, N}$, which is an ideal of $\mathfrak{t}_{n, N}$. The map $x \mapsto x^{0, \ldots, n-1}$ is an injection $\mathfrak{t}_{n-1, N} \hookrightarrow \mathfrak{t}_{n, N}$. This map induces an action of $\mathfrak{t}_{n-1, N}$ by derivations on $\mathfrak{f}_{n, N}$, and $\mathfrak{t}_{n, N}$ is the corresponding semidirect product.

When $n=2, \mathfrak{t}_{2, N}$ can be described more simply as the direct sum of its center, generated by $t_{0}^{01}+t_{0}^{02}+\sum_{a \in \mathbb{Z} / N \mathbb{Z}} t(a)^{12}$, with the free Lie algebra $\mathfrak{t}_{2, N}^{0}$ generated by $t_{0}^{01}$ and the $t(a)^{12}$ $(a \in \mathbb{Z} / N \mathbb{Z})$. We will use the following notation for generators of $\mathfrak{t}_{2, N}^{0}: A:=t_{0}^{01}, b(a):=t(a)^{12}$, $C:=$ the image $t_{0}^{02}$ by the projection $\mathfrak{t}_{2, N} \rightarrow \mathfrak{t}_{2, N}^{0}$, so $A+C+\sum_{a \in \mathbb{Z} / N \mathbb{Z}} b(a)=0$. 
In general, the element $\sum_{0<i<j<n} t_{0}^{i j}$ is central in $\mathfrak{t}_{n, N}$, where $t_{0}^{i j}:=\sum_{a \in \mathbb{Z} / N \mathbb{Z}} t(a)^{i j}$ for $j>i \geq 1$. If $\mathfrak{t}_{n, N}^{0}$ is the Lie algebra with the same generators (except $t_{0}^{0 n}$ ) and relations as $\mathfrak{t}_{n, N}$ (except those involving $\left.t_{0}^{0 n}\right)$, then $\mathfrak{t}_{n, N}^{0} \hookrightarrow \mathfrak{t}_{n, N}$ and $\mathfrak{t}_{n, N}=\mathfrak{t}_{n, N}^{0} \oplus \mathbf{k}\left(\sum_{0 \leq i<j \leq n} t_{0}^{i j}\right)$.

2.2. Definition and properties of $\Psi_{\mathrm{KZ}}$. We define $\Psi_{\mathrm{KZ}}$ as the renormalized holonomy from 0 to 1 of the differential equation

$$
\frac{\mathrm{d}}{\mathrm{d} z} H(z)=\left(\frac{t_{0}^{01}}{z}+\sum_{\zeta \in \mu_{N}(\mathbb{C})} \frac{t[\zeta]^{12}}{z-\zeta}\right) H(z),
$$

i.e., $\Psi_{\mathrm{KZ}}=H_{1^{-}}^{-1} H_{0^{+}}$, where $H_{0^{+}}, H_{1^{-}}$are the solutions such that $H_{0^{+}}(z) \sim z^{t_{0}^{01}}$ when $z \rightarrow 0^{+}$ and $H_{1^{-}}(z) \sim z^{t[1]^{12}}$ when $z \rightarrow 1^{-}$. Then $\Psi_{\mathrm{KZ}}$ belongs to $\exp \left(\hat{\mathfrak{t}}_{2, N}^{0}(\mathbb{C})\right)$.

Recall that $\Phi_{\mathrm{KZ}} \in \exp \left(\hat{\mathfrak{t}}_{3}(\mathbb{C})\right)$ is the renormalized holonomy from 0 to 1 of $G^{\prime}(z)=\left(\frac{t^{12}}{z}+\right.$ $\left.\frac{t^{12}}{z-1}\right) G(z)$, i.e., $\Phi_{\mathrm{KZ}}=G_{0^{+}}^{-1} G_{1^{-}}$, where $G_{0^{+}}, G_{1^{-}}$are the solutions such that $G_{0^{+}}(z) \sim z^{t^{12}}$ when $z \rightarrow 0^{+}$and $G_{1^{-}}(z) \sim(1-z)^{t^{23}}$ when $z \rightarrow 1^{-}$. Recall that

$$
\Phi_{\mathrm{KZ}}(V, U)=\Phi_{\mathrm{KZ}}(U, V)^{-1}, e^{\pi \mathrm{i} U} \Phi_{\mathrm{KZ}}(W, U) e^{\pi \mathrm{i} W} \Phi_{\mathrm{KZ}}(V, W) e^{\pi \mathrm{i} V} \Phi_{\mathrm{KZ}}(U, V)=1,
$$

where $U=\overline{t^{12}} \in \mathfrak{t}_{3}^{0}:=\mathfrak{t}_{3} /\left(t^{12}+t^{13}+t^{23}\right), V=\overline{t^{23}} \in \mathfrak{t}_{3}^{0}$ and $U+V+W=0$, and

$$
\Phi_{\mathrm{KZ}}^{12,3,4} \Phi_{\mathrm{KZ}}^{1,2,34}=\Phi_{\mathrm{KZ}}^{2,3,4} \Phi_{\mathrm{KZ}}^{1,23,4} \Phi_{\mathrm{KZ}}^{1,2,3}
$$

(relation in $\left.\exp \left(\hat{\mathfrak{t}}_{4}(\mathbb{C})\right)\right)$.

Proposition 2.1. The pair $\left(\Phi_{\mathrm{KZ}}, \Psi_{\mathrm{KZ}}\right)$ satisfies the mixed pentagon relation

$$
\Psi_{\mathrm{KZ}}^{12,3,4} \Psi_{\mathrm{KZ}}^{1,2,34}=\Phi_{\mathrm{KZ}}^{2,3,4} \Psi_{\mathrm{KZ}}^{1,23,4} \Psi_{\mathrm{KZ}}^{1,2,3} .
$$

in $\exp \left(\hat{\mathfrak{t}}_{3, N}^{0}(\mathbb{C})\right)$ and the octogon relation

$$
\begin{aligned}
& \Psi_{\mathrm{KZ}}(A \mid b(0), b(1), \ldots)^{-1} e^{\pi \mathrm{i} b(0)} \Psi_{\mathrm{KZ}}(C \mid b(0), b(-1), \ldots) e^{(2 \pi \mathrm{i} / N) C} \\
& \Psi_{\mathrm{KZ}}(C \mid b(1), b(0), b(-1), \ldots)^{-1} e^{\pi \mathrm{i} b(1)} \Psi_{\mathrm{KZ}}(A \mid b(1), b(2), \ldots) e^{(2 \pi \mathrm{i} / N) A}=1
\end{aligned}
$$

in $\exp \left(\hat{\mathfrak{t}}_{2, N}^{0}(\mathbb{C})\right)$.

Proof. The first equation follows as in [EE] from the the flatness of the system

$$
\begin{aligned}
\frac{\partial}{\partial z} H & =\left(\frac{t_{0}^{12}}{z}+\sum_{\zeta \in \mu_{N}(\mathbb{C})} \frac{t[\zeta]^{23}}{z-\zeta w}+\sum_{\zeta \in \mu_{N}(\mathbb{C})} \frac{t[\zeta]^{24}}{z-\zeta}\right) H, \\
\frac{\partial}{\partial w} H & =\left(\frac{t_{0}^{13}}{w}+\sum_{\zeta \in \mu_{N}(\mathbb{C})} \frac{t[\zeta]^{32}}{w-\zeta z}+\sum_{\zeta \in \mu_{N}(\mathbb{C})} \frac{t[\zeta]^{34}}{w-\zeta}\right) H .
\end{aligned}
$$

Let us prove the second equation. Let us denote by $\mathbf{D}$ the domain of $\mathbb{C}$ of all complex numbers $z$, such that $0 \leq \arg (z) \leq 2 \pi / N$, and $z \neq 0,1, e^{2 \pi \mathrm{i} / N}$. We set $\zeta_{N}=e^{2 \pi \mathrm{i} / N}$.

Recall that (4) may be rewritten $H^{\prime}(z)=\left(\frac{A}{z}+\sum_{a \in \mathbb{Z} / N \mathbb{Z}} \frac{b(a)}{z-\zeta_{N}^{a}}\right) H(z)$. There are unique solutions $H_{0^{+}}, H_{1^{-}}, H_{1^{+}}, H_{\infty}, H_{\zeta_{N} \infty}, H_{\zeta_{N}^{+}}, H_{\zeta_{N}^{-}}, H_{\zeta_{N} 0^{+}}$of $(4)$ in $\mathbf{D}$, with the following asymptotic behaviors: $H_{0^{+}}(z) \sim z^{A}$ when $z \rightarrow 0^{+}, H_{1^{-}}(z) \sim(1-z)^{b(0)}$ when $z \rightarrow 1^{-}$, $H_{1^{+}}(z) \sim(z-1)^{b(0)}$ when $z \rightarrow 1^{+}, H_{\infty}(z) \sim z^{-C}$ when $z \rightarrow+\infty$ on the real axis, $H_{\zeta_{N} \infty}(z) \sim$ $\left(z / \zeta_{N}\right)^{-C}$ when $z / \zeta \rightarrow+\infty$ on the real axis, $H_{\zeta_{N}^{+}}(z) \sim\left(\frac{z}{\zeta_{N}}-1\right)^{b(1)}$ when $z \rightarrow \zeta_{N}$ in such a way that $z / \zeta_{N}>1, H_{\zeta_{N}^{-}}(z) \sim\left(1-\frac{z}{\zeta_{N}}\right)^{b(1)}$ when $z \rightarrow \zeta_{N}$ in such a way that $z / \zeta_{N}<1$, $H_{\zeta_{N} 0^{+}}(z) \sim\left(z / \zeta_{N}\right)^{A}$ as $z \rightarrow 0$ in such a way that $z / \zeta_{N}>0$.

We have:

$$
\forall z \in] 0,1\left[, H_{0^{+}}(z)=H_{0}(z), \quad H_{1^{-}}(z)=H_{1}(z)\right.
$$




$$
\begin{gathered}
\forall z \in] 1,+\infty\left[, H_{1^{+}}(z)=H_{1}(1 / z)^{1,3,2}, \quad H_{\infty}(z)=H_{0}(1 / z)^{1,3,2},\right. \\
\forall z \in] \zeta_{N}, \zeta_{N} \infty\left[, H_{\zeta_{N} \infty}(z)=\operatorname{Ad}\left(s_{1}\right)\left(H_{0}\left(\zeta_{N} / z\right)^{1,3,2}\right), \quad H_{\zeta_{N}^{+}}(z)=\operatorname{Ad}\left(s_{1}\right)\left(H_{1}\left(\zeta_{N} / z\right)^{1,3,2}\right),\right. \\
\forall z \in] 0, \zeta_{N}\left[, H_{\zeta_{N}^{-}}(z)=\operatorname{Ad}\left(s_{1}\right)\left(H_{1}\left(z / \zeta_{N}\right)\right), \quad H_{\zeta_{N} 0^{+}}(z)=\operatorname{Ad}\left(s_{1}\right)\left(H_{0}\left(z / \zeta_{N}\right)\right) .\right.
\end{gathered}
$$

where $x \mapsto x^{1,3,2}$ and $\operatorname{Ad}\left(s_{1}\right)$ are the automorphisms of $\hat{\mathfrak{t}}_{2, N}^{0}$ given by $(A, C, b(a)) \mapsto(C, A, b(-a))$ and $(A, C, b(0), b(1), \ldots) \mapsto(A, C, b(1), b(2), \ldots)$.

It follows that $H_{1^{-}}=H_{0^{+}} \Psi_{\mathrm{KZ}}^{-1}, H_{\infty}=H_{1^{+}} \Psi_{\mathrm{KZ}}^{1,3,2}, H_{\zeta_{N}^{+}}=H_{\zeta_{N} \infty} \operatorname{Ad}\left(s_{1}\right)\left(\left(\Psi_{\mathrm{KZ}}^{-1}\right)^{1,3,2}\right), H_{\zeta_{N} 0^{+}}=$ $H_{\zeta_{N}^{-}} \operatorname{Ad}\left(s_{1}\right)\left(\Psi_{\mathrm{KZ}}\right)$.

Moreover, local study at the points $0,1, \infty, \zeta_{N}$ implies that $H_{1^{+}}=H_{1^{-}} e^{\pi \mathrm{i} b(0)}, H_{\zeta_{N} \infty}=$ $H_{\infty} e^{(2 \pi \mathrm{i} / N) C}, H_{\zeta_{N}^{-}}=H_{\zeta_{N}^{+}} e^{\pi \mathrm{i} b(1)}, H_{0^{+}}=H_{\zeta_{N} 0^{+}} e^{(2 \pi \mathrm{i} / N) A}$.

Therefore

$$
H_{0^{+}}=H_{0^{+}} \cdot \Psi_{\mathrm{KZ}}^{-1} e^{\pi \mathrm{i} b(0)} \Psi_{\mathrm{KZ}}^{1,3,2} e^{(2 \pi \mathrm{i} / N) C} \operatorname{Ad}\left(s_{1}\right)\left(\left(\Psi_{\mathrm{KZ}}^{-1}\right)^{1,3,2}\right) e^{\pi \mathrm{i} b(1)} \operatorname{Ad}\left(s_{1}\right)\left(\Psi_{\mathrm{KZ}}\right) e^{(2 \pi \mathrm{i} / N) A}=1 .
$$

Since $H_{0^{+}}$is invertible, this implies that the right factor of $H_{0^{+}}$in this expression in 1, i.e., the second relation.

Moreover, the formality isomorphism of Section 1 may be expressed algebraically in terms of $\left(\Phi_{\mathrm{KZ}}, \Psi_{\mathrm{KZ}}\right)$ as follows. The group $B_{n}^{1}$ has the following presentation: generators are $\tau, \sigma_{1}, \ldots, \sigma_{n-1}$ and relations are

$$
\begin{gathered}
\sigma_{i} \sigma_{j}=\sigma_{j} \sigma_{i}(|i-j| \geq 2), \quad \sigma_{i} \sigma_{i+1} \sigma_{i}=\sigma_{i+1} \sigma_{i} \sigma_{i+1}(i \in[1, n-1]), \\
\tau \sigma_{i}=\sigma_{i} \tau(i \in[2, n]), \quad\left(\tau \sigma_{1}\right)^{2}=\left(\sigma_{1} \tau\right)^{2} .
\end{gathered}
$$

The inclusion $B_{n}^{1} \subset B_{n+1}$ is then induced by $\sigma_{i} \mapsto \sigma_{i}, \tau \mapsto \sigma_{0}^{2}$ (the generators of $B_{n+1}$ are called $\left.\sigma_{0}, \ldots, \sigma_{n-1}\right)$.

The monodromy of the solution $F\left(z_{1}, \ldots, z_{n}\right)$ at order $n$ can be related to to that of the solution at order 2. It follows that the monodromy morphism $\mu_{n}: B_{n}^{1} \rightarrow \exp \left(\hat{\mathfrak{t}}_{n, N}(\mathbb{C})\right) \rtimes$ $(\mathbb{Z} / N \mathbb{Z})^{n} \rtimes \mathfrak{S}_{n}$ is given by

$$
\tau \mapsto e^{(2 \pi \mathrm{i} / N) t_{0}^{01}} s_{1}^{-1}, \quad \sigma_{i} \mapsto\left(\Psi_{\mathrm{KZ}}^{-1}\right)^{0 \ldots i-1, i, i+1}(i, i+1) e^{\pi \mathrm{i} t(0)^{i, i+1}} \Psi_{\mathrm{KZ}}^{0 \ldots i-1, i, i+1}
$$

for $i=1, \ldots, n-1$.

2.3. The schemes Pseudo $(N, \mathbf{k}) \subset \underline{\operatorname{Pseudo}}(N, \mathbf{k})$ and formality isomorphisms. Let $\mathbf{k}$ be a ring over $\mathbb{Q}$. Recall that $\underline{\operatorname{Assoc}}(\mathbf{k})$ is defined as the set of pairs $(\lambda, \Phi)$, where $\lambda \in \mathbf{k}$ and $\Phi \in \exp \left(\hat{\mathfrak{t}}_{3}^{0}(\mathbf{k})\right)$ satisfies

$$
\Phi(V, U)=\Phi(U, V)^{-1}, \quad e^{\lambda U / 2} \Phi(W, U) e^{\lambda W / 2} \Phi(V, W) e^{\lambda V / 2} \Phi(U, V)=1
$$

where $U, V$ generate a free Lie algebra and $U+V+W=0$, and

$$
\Phi^{1,2,34} \Phi^{12,3,4}=\Phi^{2,3,4} \Phi^{1,23,4} \Phi^{1,2,3} .
$$

We then define the set of cyclotomic associators $\underline{\operatorname{Pseudo}}(N, \mathbf{k})$ as the set of quadruples $(a, \lambda, \Phi, \Psi) \in(\mathbb{Z} / N \mathbb{Z} \times \mathbf{k}) \times \exp \left(\hat{\mathfrak{t}}_{3}^{0}(\mathbf{k})\right) \times \exp \left(\hat{\mathfrak{t}}_{2, N}^{0}(\mathbf{k})\right)$, such that $(\lambda, \Phi) \in \underline{\mathbf{A s s o c}}(\mathbf{k})$ and $\Psi$ satisfies

$$
\begin{gathered}
\Psi(A \mid b(a), b(a+1), \ldots, b(a+N-1))^{-1} e^{(\lambda / 2) b(a)} \Psi(C \mid b(a), b(a-1), \ldots, b(a+1-N)) e^{(\lambda / N) C} \\
\Psi(C \mid b(0), b(-1), \ldots, b(1-N))^{-1} e^{(\lambda / 2) b(0)} \Psi(A \mid b(0), b(1), \ldots, b(N-1)) e^{(\lambda / N) A}=1, \\
\Psi^{1,2,34} \Psi^{12,3,4}=\Phi^{2,3,4} \Psi^{1,23,4} \Psi^{1,2,3} .
\end{gathered}
$$

We also set $\boldsymbol{A s s o c}(\mathbf{k}):=\left\{(\lambda, \Phi) \in \underline{\operatorname{Assoc}}(\mathbf{k}) \mid \lambda \in \mathbf{k}^{\times}\right\}$, and $\operatorname{Pseudo}(N, \mathbf{k}):=\{(a, \lambda, \Phi, \Psi) \in$ $\left.\underline{\text { Pseudo }}(N, \mathbf{k}) \mid(a, \lambda) \in(\mathbb{Z} / N \mathbb{Z})^{\times} \times \mathbf{k}^{\times}\right\}$. If $(a, \lambda) \in(\mathbb{Z} / N \mathbb{Z}) \times \mathbf{k}$, we also set $\underline{\text { Pseudo }}_{(a, \lambda)}(N, \mathbf{k}):=$ 
$\{(\Phi, \Psi) \mid(a, \lambda, \Phi, \Psi) \in \underline{\mathbf{P s e u d o}}(N, \mathbf{k})\}$ (we write $\mathbf{P s e u d o}(a, \lambda)(N, \mathbf{k})$ instead of $\underline{\mathbf{P s e u d o}}_{(a, \lambda)}(N, \mathbf{k})$ if $\left.(a, \lambda) \in(\mathbb{Z} / N \mathbb{Z})^{\times} \times \mathbf{k}^{\times}\right)$.

Then $\Phi_{\mathrm{KZ}} \in \operatorname{Assoc}_{2 \pi \mathrm{i}}(\mathbb{C})$, and $\left(\Phi_{\mathrm{KZ}}, \Psi_{\mathrm{KZ}}\right) \in \mathbf{P s e u d o}_{(-\overline{1}, 2 \pi \mathrm{i})}(N, \mathbb{C})$.

Proposition 2.2. There is a unique map

$$
\underline{\operatorname{Pseudo}}(N, \mathbf{k}) \rightarrow \operatorname{Mor}\left(B_{n}^{1}, \exp \left(\hat{\mathfrak{t}}_{n, N}(\mathbf{k})\right) \rtimes(\mathbb{Z} / N \mathbb{Z})^{n} \rtimes \mathfrak{S}_{n}\right),
$$

taking $(a, \lambda, \Phi, \Psi)$ to the morphism

$$
\tau \mapsto e^{(\lambda / N) t_{0}^{01}} s_{1}^{a}, \quad \sigma_{i} \mapsto\left(\Psi^{-1}\right)^{0 \ldots i-1, i, i+1}(i, i+1) e^{(\lambda / 2) t(0)^{i, i+1}} \Psi_{\mathrm{KZ}}^{0 \ldots i-1, i, i+1}
$$

for $i=1, \ldots, n-1$. When $(a, \lambda) \in(\mathbb{Z} / N \mathbb{Z})^{\times} \times \mathbf{k}^{\times}$, this morphism induces an isomorphism $B_{n}^{1}\left(\mathbf{k}, \varphi_{N}\right) \rightarrow \exp \left(\hat{\mathfrak{t}}_{n, N}(\mathbf{k})\right) \rtimes(\mathbb{Z} / N \mathbb{Z})^{n} \rtimes \mathfrak{S}_{n}$, hence we get a map

$$
\operatorname{Pseudo}(N, \mathbf{k}) \rightarrow \operatorname{Iso}\left(B_{n}^{1}\left(\varphi_{N}, \mathbf{k}\right), \exp \left(\hat{\mathfrak{t}}_{n, N}(\mathbf{k})\right) \rtimes(\mathbb{Z} / N \mathbb{Z})^{n} \rtimes \mathfrak{S}_{n}\right) .
$$

Proof. The proof of the fact that the above formulas define a group morphism follows the construction of representations of $B_{n}^{1}$ in Section 4. One studies the image of the generators $X_{0 i}$, $x_{i j}(a)$ to prove that we get an isomorphism when $(a, \lambda) \in(\mathbb{Z} / N \mathbb{Z})^{\times} \times \mathbf{k}^{\times}$.

We will see later that $\operatorname{Pseudo}(N, \mathbf{k})$ is a torsor; one can prove that the above map is a morphism of torsors. To prove that a formality isomorphism $B_{n}^{1}\left(\varphi_{N}, \mathbb{Q}\right) \simeq \exp \left(\hat{\mathfrak{t}}_{n, N}(\mathbb{Q})\right) \rtimes$ $(\mathbb{Z} / N \mathbb{Z})^{n} \rtimes \mathfrak{S}_{n}$ defined over $\mathbb{Q}$ exists, we will show that $\operatorname{Pseudo}_{(a, \lambda)}(N, \mathbb{Q}) \neq \emptyset$ for any $(a, \lambda) \in$ $(\mathbb{Z} / N \mathbb{Z})^{\times} \times \mathbf{k}^{\times}$, which will be based on the action of a group $\operatorname{GTM}(N, \mathbf{k})$. The two next sections serve as a motivation for the definition of $\operatorname{GTM}(N, \mathbf{k})$, which is the group of automorphisms of certain braided module categories.

\section{Pseudotwists over quasibialgebras and module categories}

In this section, we recall the notion of a pseudotwist over a quasi-bialgebra ${ }^{3}$ and relate it to the notion of module category over a monoidal category.

3.1. Pseudotwists. Let $\left(A, \Delta_{A}, \Phi_{A}\right)$ be a quasibialgebra (QBA). Recall that this means that $A$ is an algebra, $\Delta_{A}: A \rightarrow A^{\otimes 2}$ is a morphism, $\Phi_{A} \in A^{\otimes 3}$ is invertible, and

$$
\begin{gathered}
\forall a \in A,\left(\operatorname{id}_{A} \otimes \Delta_{A}\right)\left(\Delta_{A}(a)\right)=\Phi_{A}\left(\Delta_{A} \otimes \operatorname{id}_{A}\right)\left(\Delta_{A}(a)\right) \Phi_{A}^{-1} \\
\Phi_{A}^{1,2,34} \Phi_{A}^{12,3,4}=\Phi_{A}^{2,3,4} \Phi_{A}^{1,23,4} \Phi_{A}^{1,2,3} .
\end{gathered}
$$

The counit $\varepsilon_{A}$ is also required to satisfy $\left(\varepsilon_{A} \otimes \mathrm{id}_{A}\right) \circ \Delta_{A}=\left(\mathrm{id}_{A} \otimes \varepsilon_{A}\right) \circ \Delta_{A}=\mathrm{id}_{A}, \varepsilon_{A}^{i}\left(\Phi_{A}\right)=1^{\otimes 2}$ if $i=1,2,3$ (here $\varepsilon_{A}^{1}=\varepsilon_{A} \otimes \mathrm{id}_{A}^{\otimes 2}$, etc.; see [Dr1]).

Definition 3.1. A pseudotwist over $A$ is a triple $\left(B, \Delta_{B}, \Psi_{B}\right)$ of an algebra $B$, an algebra morphism $\Delta_{B}: B \rightarrow B \otimes A$ and an invertible $\Psi_{B} \in B \otimes A^{\otimes 2}$, such that $\left(\mathrm{id}_{B} \otimes \varepsilon_{A}\right) \circ \Delta_{B}=\mathrm{id}_{B}$ and

$$
\begin{gathered}
\forall b \in B,\left(\operatorname{id}_{B} \otimes \Delta_{A}\right)\left(\Delta_{B}(b)\right)=\Psi_{B}\left(\Delta_{B} \otimes \operatorname{id}_{A}\right)\left(\Delta_{B}(b)\right) \Psi_{B}^{-1}, \\
\Psi_{B}^{1,2,34} \Psi_{B}^{12,3,4}=\Phi_{A}^{2,3,4} \Psi_{B}^{1,23,4} \Psi_{B}^{1,2,3} .
\end{gathered}
$$

Here $\Psi_{B}^{12,3,4}=\left(\Delta_{B} \otimes \operatorname{id}_{A}^{\otimes 2}\right)\left(\Psi_{B}\right), \Psi_{B}^{1,23,4}=\left(\operatorname{id}_{B} \otimes \Delta_{A} \otimes \operatorname{id}_{A}\right)\left(\Psi_{B}\right)$, etc.

So the notion of pseudotwist is the "quasi" version of that of comodule-algebra over a bialgebra $A$ (which corresponds to $\Phi_{A}=1, \Psi_{B}=1$ ).

\footnotetext{
${ }^{3}$ All (co)algebras are understood to be with (co)unit. We use the standard notation for multiple coproducts in coalgebras, so $x^{12,34}=(\Delta \otimes \Delta)(x)$, etc. If $A$ is an algebra, we denote by $A^{\times}$the group of invertible elements of $A$.
} 
BENJAMIN ENRIQUEZ

Remark 3.2. Similarly to [EN], Propositions 2.4 and 2.6, one proves that if $B \subset A$ is a subalgebra such that $\Delta_{A}(B) \subset B \otimes A$, there is a bijection between the following sets: (a) invertible elements $\Psi_{B} \in B \otimes A^{\otimes 2}$, satisfying (12) and (13), where $\Delta_{B}:=\left(\Delta_{A}\right)_{\mid B}$; and (b) invertible elements $\Upsilon \in A^{\otimes 2}$, such that $\forall b \in B, \Upsilon \Delta_{A}(b)=\Delta_{A}(b) \Upsilon$, and $\Upsilon * \Phi_{A}:=\left(\Upsilon^{1,23}\right)^{-1} \Phi_{A} \Upsilon^{12,3} \Upsilon^{1,2} \in$ $B \otimes A^{\otimes 2}$. This bijection takes $\Psi_{B}$ to $\Upsilon:=\left(\varepsilon_{A} \otimes \mathrm{id}_{A}^{\otimes 2}\right)\left(\Psi_{B}\right)$, and its inverse takes $\Upsilon$ to $\Upsilon * \Phi_{A}$.

3.2. Module categories over monoidal categories. Let $\left(\mathcal{C}, \otimes, \Phi_{\mathcal{C}}, \mathbf{1}, l, r\right)$ be a monoidal category. Recall that this means that $\mathcal{C}$ is k-linear, $\otimes: \mathcal{C}^{2} \rightarrow \mathcal{C}$ is a bifunctor, $\Phi_{\mathcal{C}}$ is a functorial and additive assignment ${ }^{4} \Phi_{X, Y, Z} \in \operatorname{Iso}_{\mathcal{C}}(X \otimes(Y \otimes Z),(X \otimes Y) \otimes Z)$, satisfying the pentagon identity, $\mathbf{1} \in \mathrm{Ob}(\mathcal{C})$ and $l, r$ are additive and functorial isomorphisms $l_{X} \in \operatorname{Iso}_{\mathcal{C}}(\mathbf{1} \otimes X, X)$, $r_{X} \in \operatorname{Iso}_{\mathcal{C}}(X \otimes \mathbf{1}, X)$, compatible with $\Phi_{\mathcal{C}}$.

Let $\left(\mathcal{M}, \otimes, \Psi_{\mathcal{M}}, l^{\prime}\right)$ be a right module category over $\mathcal{C}([\mathrm{O}, \mathrm{CF}])$. This means that $\mathcal{M}$ is k-linear, $\otimes: \mathcal{M} \times \mathcal{C} \rightarrow \mathcal{M}$ is a bifunctor, $\Psi_{\mathcal{M}}$ is a functorial and additive assignment $\Psi_{M, X, Y} \in$ $\operatorname{Iso}_{\mathcal{M}}(M \otimes(X \otimes Y),(M \otimes X) \otimes Y)$ for $M \in \mathrm{Ob}(M), X, Y \in \mathrm{Ob}(\mathcal{C})$, and $r^{\prime}$ is a functorial and additive assignment $r_{M}^{\prime} \in \operatorname{Iso}_{\mathcal{M}}(M \otimes \mathbf{1}, M)$, such that $\Psi_{M \otimes X, Y, Z} \circ \Psi_{M, X, Y \otimes Z}=\left(\Psi_{M, X, Y} \otimes\right.$ $\left.\operatorname{id}_{Z}\right) \circ \Psi_{M, X \otimes Y, Z} \circ\left(\operatorname{id}_{M} \otimes \Phi_{X, Y, Z}\right)$ (mixed pentagon), and $\left(r_{M \otimes X} \otimes \operatorname{id}_{\mathbf{1}}\right) \circ \Psi_{M, X, \mathbf{1}}=\operatorname{id}_{M} \otimes r_{X}$, $\left(r_{M}^{\prime} \otimes \operatorname{id}_{X}\right) \circ \Psi_{M, \mathbf{1}, X}=\operatorname{id}_{M} \otimes l_{X}$.

If $\left(B, \Delta_{B}, \Psi_{B}\right)$ is a pseudotwist over the QBA $\left(A, \Delta_{A}, \Phi_{A}\right)$, then $\mathcal{M}:=\operatorname{Rep}(B)$ is a right module category over $\mathcal{C}:=\operatorname{Rep}(A)$.

3.3. A coherence theorem for module categories. Let us define the groupoid $\mathbf{P a} \mathbf{a}_{n}$ of parenthesizations of $n$ letters as follows (here $n \geq 1$ ). We set $\mathrm{Ob}\left(\mathbf{P} \mathbf{a}_{n}\right):=\{$ parenthesizations of the word $\left.x_{1} \ldots x_{n}\right\}$. Let us define the morphisms of $\mathbf{P} \mathbf{a}_{n}$. If $w^{\prime}, w^{\prime \prime}, w^{\prime \prime \prime}$ are parenthesizations of $x_{a} \ldots x_{b}, x_{b+1} \ldots x_{c}$ and $x_{c+1} \ldots x_{d}$, if $w_{0}$ contains $w^{\prime}\left(w^{\prime \prime} w^{\prime \prime \prime}\right)$ and $w_{1}$ is obtained from $w_{0}$ by the replacement $w^{\prime}\left(w^{\prime \prime} w^{\prime \prime \prime}\right) \rightarrow\left(w^{\prime} w^{\prime \prime}\right) w^{\prime \prime}$, then there is an invertible morphism $a\left(w^{\prime}, w^{\prime \prime}, w^{\prime \prime \prime}\right) \in$ $\mathbf{P a}_{n}\left(w_{0}, w_{1}\right)$; then $\mathbf{P} \mathbf{a}_{n}$ is the free groupoid generated by these morphisms and their inverses.

Let $\mathcal{C}$ be a monoidal category. To $X_{1}, \ldots, X_{n} \in \mathrm{Ob}(\mathcal{C})$, we attach a representation of $\mathbf{P a} \mathbf{a}_{n}$; the space corresponding to $w \in \mathrm{Ob}\left(\mathbf{P a}_{n}\right)$ is $\otimes_{1<i<n}^{w} X_{i}$ (meaning that the order in which tensor products are taken follows $w)$. The image of $\bar{a}\left(w^{\prime}, w^{\prime \prime}, w^{\prime \prime \prime}\right)$ is $\mathrm{id} \ldots \otimes \Phi_{X^{\prime}, X^{\prime \prime}, X^{\prime \prime \prime}} \otimes \mathrm{id} \ldots$, where $X^{\prime}:=\otimes_{1 \leq i \leq a}^{w^{\prime}} X_{i}, X^{\prime \prime}:=\otimes_{a+1 \leq i \leq b}^{w^{\prime \prime}} X_{i}, X^{\prime \prime \prime}:=\otimes_{b+1 \leq i \leq n}^{w^{\prime \prime \prime}} X_{i}$. Then McLane's theorem says that this representation is trivial (i.e., its restriction to each $\mathbf{P a}_{n}(w, w)$ is trivial).

Let now $\mathcal{M}$ be a module category over $\mathcal{C}$. To $M \in \mathrm{Ob}(\mathcal{M})$ and $X_{1}, \ldots, X_{n} \in \mathrm{Ob}(\mathcal{C})$, we attach a representation of $\mathbf{P} \mathbf{a}_{n+1}$ as above (the $\Phi_{\ldots}$ are replaced by $\Psi \ldots$ when $X^{\prime}$ contains $M$ ). One can check following the proof of McLane's theorem that this representation is trivial.

\section{QuAsi-REFlection ALGEBRAs AND BRAided MODUle CATEGORIES}

In this section, we introduce the notion of quasi-reflection algebras (QRA's) over a quasitriangular quasi-bialgebra (QTQBA). The corresponding notion is that of braided module category over a monoidal category. We show that such objects give rise to representations of $B_{n}^{1}$.

4.1. QRA's over QTQBA's. Let $\left(A, \Delta_{A}, R, \Phi_{A}, \varepsilon_{A}\right)$ be a quasitriangular quasibialgebra. This means that $A$ is an algebra, $\Delta_{A}: A \rightarrow A^{\otimes 2}$ is a morphism, $R \in A^{\otimes 2}$ and $\Phi_{A} \in A^{\otimes 3}$ are invertible, and

$$
\begin{gathered}
\forall a \in A, \Delta_{A}^{2,1}(a)=R \Delta_{A}(a) R^{-1},\left(\operatorname{id}_{A} \otimes \Delta_{A}\right) \circ \Delta_{A}(a)=\Phi_{A}\left(\Delta_{A} \otimes \operatorname{id}_{A}\right) \circ \Delta_{A}(a) \Phi_{A}^{-1} \\
\Phi_{A}^{1,2,34} \Phi_{A}^{12,3,4}=\Phi_{A}^{2,3,4} \Phi_{A}^{1,23,4} \Phi_{A}^{1,2,3} \\
R^{12,3}=\Phi_{A}^{3,1,2} R^{1,3}\left(\Phi_{A}^{1,3,2}\right)^{-1} R^{2,3} \Phi_{A}, R^{1,23}=\left(\Phi_{A}^{2,3,1}\right)^{-1} R^{1,3} \Phi_{A}^{2,1,3} R^{1,2} \Phi_{A}^{-1} .
\end{gathered}
$$

\footnotetext{
${ }^{4}$ If $\mathcal{C}$ is a category, we denote by $\mathcal{C}(X, Y)$ the set of morphisms from $X$ to $Y$, by $\operatorname{Iso}_{\mathcal{C}}(X, Y) \subset \mathcal{C}(X, Y)$ the subset of isomorphisms and by $\operatorname{Aut}_{\mathcal{C}}(X)=\operatorname{Iso}_{\mathcal{C}}(X, X)$ the set of automorphisms of $X$.
} 
These conditions are the pentagon and the two hexagon equations. The counit $\varepsilon_{A}$ satisfies $\left(\varepsilon_{A} \otimes \operatorname{id}_{A}\right) \circ \Delta_{A}=\left(\operatorname{id}_{A} \otimes \varepsilon_{A}\right) \circ \Delta_{A}=\operatorname{id}_{A}, \varepsilon_{A}^{i}(R)=1$ for $i=1,2, \varepsilon_{A}^{i}\left(\Phi_{A}\right)=1^{\otimes 2}$ if $i=1,2,3$.

Definition 4.1. A quasi-reflection algebra (QRA) over $A$ is a quadruple $\left(B, \Delta_{B}, E, \Psi_{B}\right)$, where $\left(B, \Delta_{B}, \Psi_{B}\right)$ is a pseudotwist over $A$, and $E$ satisfies

$$
\left(\Delta_{B} \otimes \operatorname{id}_{A}\right)(E)=\Psi_{B}^{-1} R^{3,2} \Psi_{B}^{1,3,2} E^{1,3}\left(\Psi_{B}^{1,3,2}\right)^{-1} R^{2,3} \Psi_{B} .
$$

We will call the relations (13) and (14) the mixed pentagon and octogon relations.

When $\Phi_{A}=1, A$ is a quasitriangular bialgebra, and when $\Psi_{B}=1$, we say that $B$ is a reflection algebra (RA) over $A$.

Remark 4.2. If $A$ is a QTQBA and $B \subset A$ be a subalgebra such that $\Delta_{A}(B) \subset B \otimes A$, and set $\Delta_{B}:=\left(\Delta_{A}\right)_{\mid B}$. One can show that there is a bijection between the following sets: (a) pairs $\left(E, \Psi_{B}\right)$, where $E \in B \otimes A$ and $\Psi_{B} \in B \otimes A^{\otimes 2}$ are invertible, s.t. $\left(B, \Delta_{B}, E, \Psi_{B}\right)$ is a QRA over $A$; (b) pairs $(\Sigma, \Upsilon)$, where $\Sigma \in A$ and $\Upsilon \in A^{\otimes 2}$ are invertible, such that $\forall b \in B, \Sigma b=b \Sigma$, $\Upsilon \Delta_{A}(b)=\Delta_{A}(b) \Upsilon,\left(\Upsilon^{1,23}\right)^{-1} \Phi_{A} \Upsilon^{12,3} \Upsilon^{1,2} \in B \otimes A^{\otimes 2}$ and $\Upsilon^{-1} R^{2,1} \Upsilon^{2,1} \Sigma^{2}\left(\Upsilon^{2,1}\right)^{-1} R \Upsilon \in B \otimes A$. This bijection takes $\left(E, \Psi_{B}\right)$ to $\left(\Sigma(E), \Upsilon\left(\Psi_{B}\right)\right)$, where $\Sigma(E):=\left(\varepsilon_{A} \otimes \operatorname{id}_{A}\right)(E)$ and $\Upsilon\left(\Psi_{B}\right):=$ $\left(\varepsilon_{A} \otimes \mathrm{id}_{A}^{\otimes 2}\right)\left(\Psi_{B}\right)$. The inverse bijection takes $(\Sigma, \Upsilon)$ to $(E(\Sigma, \Upsilon), \Psi(\Upsilon))$, where

$$
E(\Sigma, \Upsilon):=\Upsilon^{-1} R^{2,1} \Upsilon^{2,1} \Sigma^{2}\left(\Upsilon^{2,1}\right)^{-1} R \Upsilon, \Psi(\Upsilon):=\left(\Upsilon^{1,23}\right)^{-1} \Phi_{A} \Upsilon^{12,3} \Upsilon^{1,2}
$$

When $\Phi_{A}=1$, this restricts to a bijection between the sets of pairs $\left(E, \Psi_{B}\right)$ and $(\Sigma, \Upsilon)$ as above, where $\Psi_{B}=1$ and $\Upsilon=1$.

Remark 4.3. If $B$ is a RA over the QTBA $A$, then $(E, R)$ satisfy the reflection equation

$$
E^{1,2} R^{3,2} E^{1,3} R^{2,3}=R^{3,2} E^{1,3} R^{2,3} E^{1,2} .
$$

In particular, if $(\rho, V)$ is a $B$-module, then $K:=(\rho \otimes$ id $)(E)$ satisfies the reflection equation $R^{2,1} K^{2} R^{1,2} K^{1}=K^{1} R^{2,1} K^{2} R^{1,2}$. Reflection equation algebras (REA) are defined using this equation ([FRT]), $R$ being fixed; REA's yield representations of $B_{n}^{1}$. Applications of REA's include the topology of handlebodies $([\mathrm{KS}])$ and scattering theory with reflection $([\mathrm{Ch}])$. The analogy between both situations is that winding around the first fixed strand is analogous to the reflection of a particle on a wall. In [DKM], universal versions of REA's were studied; they rely on the datum of a "semiuniversal" object $K \in \operatorname{End}(V) \otimes B$, where $V$ is a $A$-module, $A$ is a Hopf algebra and $B$ is an algebra. The data of a RA $B$ over a QTBA $A$ and a $A$-module $V$ yield such a $K$.

Example 4.4. If we set $B:=A, E:=R^{2,1} R, \Psi_{B}:=\Phi_{A}$, then $\left(B, E, \Psi_{B}\right)$ is a QRA over $A$. It corresponds to $\Sigma=1, \Upsilon=1^{\otimes 2}$.

Example 4.5. Let $\mathfrak{g}$ be a Lie algebra, let $\Gamma$ be a group acting on $\mathfrak{g}$ by automorphisms. Set $A:=U(\mathfrak{g}) \rtimes \Gamma$. Define $\Delta_{A}: A \rightarrow A^{\otimes 2}$ as the unique algebra morphism such that $\Delta_{A}(x)=$ $x \otimes 1+1 \otimes x$ for $x \in \mathfrak{g}, \Delta_{A}(\gamma)=\gamma \otimes \gamma$ for $\gamma \in \Gamma$. Set $R:=1^{\otimes 2}$, then $\left(A, \Delta_{A}, R\right)$ is a QTBA. Fix $\sigma \in \Gamma$ and a Lie subalgebra $\mathfrak{l} \subset \mathfrak{g}^{\sigma}$. Set $B:=U(\mathfrak{l}), E:=1 \otimes \sigma$. Then $(B, E)$ is a RA over $A$. We will later introduce a deformation of this example, where $\Gamma=\mathbb{Z} / N \mathbb{Z}$.

4.2. Twists. Let $\left(A, \Delta_{A}, R, \Phi_{A}\right)$ be a QTQBA. To an invertible $F \in A^{\otimes 2}$, one associates the twisted QTQBA ${ }^{F} A:=\left(A,{ }^{F} \Delta,{ }^{F} R,{ }^{F} \Phi_{A}\right)$, where

$$
{ }^{F_{\Delta}} \Delta_{A}(a)=F \Delta_{A}(a) F^{-1},{ }^{F} R=F^{2,1} R F^{-1},{ }^{F} \Phi_{A}=F^{2,3} F^{1,23} \Phi_{A}\left(F^{1,2} F^{12,3}\right)^{-1} .
$$

If now $\left(B, E, \Psi_{B}\right)$ is a QRA over $A$ and $G \in B \otimes A$ is invertible, set

${ }^{G} \Delta_{B}(b)=G \Delta_{B}(b) G^{-1},{ }^{G} E:=G E G^{-1},{ }^{F, G} \Psi_{B}:=F^{2,3}\left(\operatorname{id}_{B} \otimes \Delta_{A}\right)(G) \Psi_{B}\left(G^{1,2}\left(\Delta_{B} \otimes \operatorname{id}_{A}\right)(G)\right)^{-1}$.

Then $\left(B,{ }^{G} \Delta_{B},{ }^{G} E,{ }^{F, G} \Psi_{B}\right)$ is a QRA over $\left(A,{ }^{F} \Delta_{A},{ }^{F} R,{ }^{F} \Phi_{A}\right)$; we call it the twist of $B$ by $(F, G)$. 
4.3. Braided module categories over braided monoidal categories. Let $\mathcal{C}$ be a braided monoidal category. This means that $\mathcal{C}$ is a monoidal category, equipped with a braiding $\beta_{X, Y} \in$ $\operatorname{Iso}_{\mathcal{C}}(X \otimes Y, Y \otimes X)($ where $X, Y \in \mathrm{Ob}(\mathcal{C})), \beta_{X \otimes Y, Z}=\left(\beta_{X, Z} \otimes \operatorname{id}_{Y}\right) \circ\left(\operatorname{id}_{X} \otimes \beta_{Y, Z}\right), \beta_{X, Y \otimes Z}=$ $\left(\operatorname{id}_{Y} \otimes \beta_{X, Z}\right) \circ\left(\beta_{X, Y} \otimes \operatorname{id}_{Z}\right), \beta_{\mathbf{1}, X}=\beta_{X, \mathbf{1}}=\operatorname{id}_{X}$ (we omitted the associativity constraints).

We define a braided module category over $\mathcal{C}$ to be a module category $\mathcal{M}$ over $\mathcal{C}$, equipped with an additive and functorial $\varepsilon_{M, X} \in \operatorname{Aut}_{\mathcal{M}}(M \otimes X)$, such that

$\varepsilon_{M \otimes X, Y}=\Psi_{M, X, Y}^{-1} \circ\left(\operatorname{id}_{M} \otimes \beta_{Y, X}\right) \circ \Psi_{M, Y, X} \circ\left(\varepsilon_{M, X} \otimes \operatorname{id}_{Y}\right) \circ \Psi_{M, Y, X}^{-1} \circ\left(\operatorname{id}_{M} \otimes \beta_{X, Y}\right) \circ \Psi_{M, X, Y}$.

Then if $A$ is a QTQBA and $B$ is a QRA over $A$, then $\mathcal{M}:=\operatorname{Rep}(B)$ is a braided module category over $\mathcal{C}:=\operatorname{Rep}(A)$; the braided monoidal structure on $\mathcal{C}$ is $\beta_{X, Y}:=\sigma_{X, Y} \circ\left(\rho_{X} \otimes \rho_{Y}\right)(R)$ and $\Phi_{X, Y, Z}:=\left(\rho_{X} \otimes \rho_{Y} \otimes \rho_{Z}\right)\left(\Phi_{A}\right)$ for $\left(X, \rho_{X}\right), \ldots \in \operatorname{Ob}(\operatorname{Rep}(A))\left(\right.$ here $\sigma_{X, Y} \in \operatorname{Hom}_{\mathbf{k}}(X \otimes Y, Y \otimes X)$ is the exchange operator) and the braided module structure on $\mathcal{M}$ is $\varepsilon_{M, X}:=\left(\rho_{M} \otimes \rho_{X}\right)(E)$, $\Psi_{M, X, Y}:=\left(\rho_{M} \otimes \rho_{X} \otimes \rho_{Y}\right)\left(\Psi_{B}\right)$ for $\left(M, \rho_{M}\right) \in \operatorname{Ob}(\operatorname{Rep}(B))$.

4.4. Braided module categories and representations of $B_{n}^{1}$. Recall that if $\mathcal{C}$ is a strict braided tensor category, and $X \in \mathrm{Ob}(\mathcal{C})$, then $\beta$ gives rise to a group morphism $B_{n} \rightarrow$ $\operatorname{Aut}_{\mathcal{C}}\left(X^{\otimes n}\right)$ for any $X \in \mathrm{Ob}(\mathcal{C})$. If $\sigma_{1}, \ldots, \sigma_{n-1}$ are the standard generators of $B_{n}$, this morphism is $\sigma_{i} \mapsto \mathrm{id}_{X \otimes i-1} \otimes \beta_{X, X} \otimes \mathrm{id}_{X \otimes n-i-1}$.

If now $\mathcal{M}$ is a strict braided module category over the strict braided monoidal category $\mathcal{C}$ (i.e., the two natural bifunctors $\mathcal{C}^{3} \rightarrow \mathcal{C}$ coincides, as well as the two bifunctors $\mathcal{M} \times \mathcal{C}^{2} \rightarrow \mathcal{M}$, and the constraints $\Phi_{\mathcal{C}}$ and $\Psi_{\mathcal{M}}$ are the identity), if $M \in \mathrm{Ob}(\mathcal{M})$ and $X \in \mathrm{Ob}(\mathcal{C})$, then $\varepsilon, \beta$ give rise to a compatible group morphism $B_{n}^{1} \rightarrow \operatorname{Aut}_{\mathcal{M}}\left(M \otimes X^{\otimes n}\right)$ for $M \in \mathrm{Ob}(\mathcal{M}), X \in \operatorname{Ob}(\mathcal{C})$, by $\tau \mapsto \varepsilon_{M, X} \otimes \operatorname{id}_{X \otimes n-1}, \sigma_{i} \mapsto \operatorname{id}_{M \otimes X \otimes i-1} \otimes \beta_{X, X} \otimes \operatorname{id}_{X \otimes n-i-1}$.

The fact that $\left(\tau \sigma_{1}\right)^{2}=\left(\sigma_{1} \tau\right)^{2}$ is preserved follows from the identity $\left(\varepsilon_{M, X} \otimes \operatorname{id}_{X}\right) \circ \varepsilon_{M \otimes X, X}=$ $\varepsilon_{M \otimes X, X} \circ\left(\varepsilon_{M, X} \otimes \operatorname{id}_{X}\right)$, which follows from the functoriality of $\varepsilon$.

In [BN], the groupoid $\mathbf{P a B}_{n}$ was defined by $\mathrm{Ob}\left(\mathbf{P a B}_{n}\right)=\left\{(\sigma, w) \mid \sigma \in \mathfrak{S}_{n}, w\right.$ is a parenthesization of $\left.x_{\sigma(1)} \ldots x_{\sigma(n)}\right\}$ and $\mathbf{P a B}_{n}\left((\sigma, w),\left(\sigma^{\prime}, w^{\prime}\right)\right)=$ fbraids relating $x_{\sigma(1)} \ldots x_{\sigma(n)}$ and

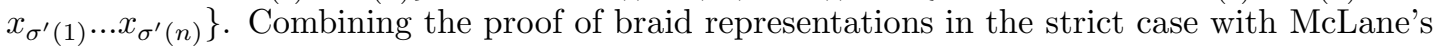
theorem, one shows that to any braided monoidal category $\mathcal{C}$ and any $X_{1}, \ldots, X_{n} \in \operatorname{Ob}(\mathcal{C})$, there corresponds a representation of $\mathbf{P a B}_{n}$, where the object corresponding to $(\sigma, w)$ is $\otimes_{1 \leq i \leq n}^{w} X_{i}$. In particular, we get a morphism $B_{n} \rightarrow \operatorname{Aut}_{\mathcal{C}}\left(X^{\otimes n}\right)$, where $X^{\otimes n}=((X \otimes X) \otimes \ldots) \otimes X$. When $\mathcal{C}=\operatorname{Rep}(A)$, where $A$ is a QTQBA, this morphism is induced by a morphism $B_{n} \rightarrow$ $\left(A^{\otimes n}\right)^{\times} \rtimes \mathfrak{S}_{n}$, such that

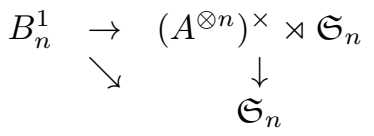

commutes; it is given by

$$
\sigma_{i} \mapsto\left(\Phi_{A}^{((12) . .) . i-1, i, i+1}\right)^{-1} \cdot(i, i+1) R^{i, i+1} \cdot \Phi_{A}^{((12) \ldots) i-1, i, i+1}
$$

(the generators of $B_{n}$ are $\left.\sigma_{1}, \ldots, \sigma_{n-1}\right)$; here $\Phi_{A}^{((12) . .) . i-1, i, i+1}=\left(\Delta_{A}^{((12) \ldots) i-1} \otimes \mathrm{id}_{A}^{\otimes 2}\right)\left(\Phi_{A}\right)$, and $\Delta_{A}^{((12) \ldots) i}=\left(\Delta_{A} \otimes \mathrm{id}_{A}^{\otimes i-2}\right) \circ \ldots \circ \Delta_{A}$.

We define similarly the groupoid $\mathbf{P a B} \mathbf{B}_{n}^{1}$ by $\mathrm{Ob}\left(\mathbf{P a B}_{n}\right)=\left\{(\sigma, w) \mid \sigma \in \mathfrak{S}_{n}, w\right.$ is a parenthesization of $\left.x_{0} x_{\sigma(1)} \ldots x_{\sigma(n)}\right\}$ and $\mathbf{P a B}_{n}\left((\sigma, w),\left(\sigma^{\prime}, w^{\prime}\right)\right)=\left\{\right.$ braids relating $x_{0} x_{\sigma(1)} \ldots x_{\sigma(n)}$ and $\left.x_{0} x_{\sigma^{\prime}(1)} \ldots x_{\sigma^{\prime}(n)}\right\}$. As above, to the data of a braided module category $\mathcal{M}$ over the braided monoidal category $\mathcal{C}$, and to $M \in \mathrm{Ob}(\mathcal{M}), X_{1}, \ldots, X_{n} \in \mathrm{Ob}(\mathcal{C})$, there corresponds a representation of $\mathbf{P a B}_{n}^{1}$, where to $(\sigma, w)$, we attach $\otimes_{0 \leq i \leq n}^{w} X_{\sigma(i)}$, where $X_{0}:=M$ and $\sigma(0)=0$. In particular, we get a morphism $B_{n}^{1} \rightarrow \operatorname{Aut}_{\mathcal{M}}\left(M \otimes X^{\otimes n}\right)$, where $M \otimes X^{\otimes n}=((M \otimes X) \otimes \ldots) \otimes X$. When $\mathcal{C}=\operatorname{Rep}(A), \mathcal{M}=\operatorname{Rep}(B), A$ is a QTQBA and $B$ is a QRA over $A$, this morphism is 
induced by a morphism $B_{n}^{1} \rightarrow(B \otimes A)^{\otimes n} \rtimes \mathfrak{S}_{n}$, such that

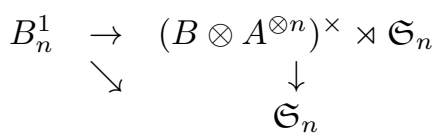

commutes; this morphism is given by

$$
\tau \mapsto E^{0,1}, \quad \sigma_{i} \mapsto\left(\Psi_{B}^{((12) \ldots) i-1, i, i+1}\right)^{-1} \cdot(i, i+1) R^{i, i+1} \cdot\left(\Psi_{B}^{((12) \ldots) i-1, i, i+1}\right)^{-1},
$$

where $\Psi_{B}^{((12) \ldots) i-1, i, i+1}=\left(\Delta_{B}^{((12) \ldots) i-1} \otimes \operatorname{id}_{A}^{\otimes 2}\right)\left(\Psi_{B}\right)$, and $\Delta_{B}^{((12) \ldots) i}=\left(\Delta_{B} \otimes \operatorname{id}_{A}^{\otimes i-2}\right) \circ \ldots \circ \Delta_{B}$.

Remark 4.6. If $g \geq 1$, let $B_{n}^{g}:=B_{g+n} \times \mathfrak{S}_{g+n} \mathfrak{S}_{n}$, where $\mathfrak{S}_{n} \subset \mathfrak{S}_{g+n}$ is the subgroup of permutations of the last $n$ elements. A presentation of $B_{n}^{g}$ can be found in [Sos, V]; generators are $\tau_{1}, \ldots, \tau_{g}, \sigma_{1}, \ldots, \sigma_{g}$ and relations are $(5), \tau_{k} \sigma_{i}=\sigma_{i} \tau_{k}(k \in[1, g], i \in[2, n]), \tau_{k} \sigma_{1} \tau_{k} \sigma_{1}=$ $\sigma_{1} \tau_{k} \sigma_{1} \tau_{k}(k \in[1, g]), \sigma_{1} \tau_{\ell} \sigma_{1}^{-1} \tau_{k}=\tau_{k} \sigma_{1} \tau_{\ell} \sigma_{1}^{-1}(1 \leq k<l \leq g)$. The inclusion $B_{n}^{g} \subset B_{n+g}$ is induced by $\sigma_{i} \mapsto \sigma_{g+i}, \tau_{i} \mapsto \sigma_{g}^{-1} \cdots \sigma_{i+1}^{-1} \sigma_{i}^{2} \sigma_{i+1} \cdots \sigma_{g}$.

If $\mathcal{C}$ is a strict braided monoidal category, $\mathcal{M}$ is a strict monoidal category and $F: \mathcal{C} \rightarrow \mathcal{M}$ is a monoidal functor, then $\mathcal{M}$ is a right strict module category over $\mathcal{C}$ (the bifunctor $\otimes$ : $\mathcal{M} \times \mathcal{C} \rightarrow \mathcal{M}$ is $(M, X) \mapsto M \otimes F(X))$. Then a braided module category structure of $\mathcal{M}$ over $\mathcal{C}$ gives rise to group morphisms $B_{n}^{g} \rightarrow \operatorname{Aut}_{\mathcal{M}}\left(\otimes_{i=1}^{g} M_{i} \otimes X^{\otimes n}\right)$. We describe these morphisms when $\mathcal{C}=\operatorname{Rep}(A), \mathcal{M}=\operatorname{Rep}(B), A$ is a QTBA, $B \subset A$ is a subbialgebra. The morphism $B_{n}^{g} \rightarrow\left(B^{\otimes g} \otimes A^{\otimes n}\right)^{\times} \rtimes \mathfrak{S}_{n}$ is given by $\sigma_{i} \mapsto(\bar{i}, \overline{i+1}) \circ R^{\bar{i}, \overline{i+1}}, \tau_{i} \mapsto\left(E^{i+1 \ldots g, \overline{1}}\right)^{-1} E^{i \ldots g, \overline{1}}$ (we set $E^{\emptyset, \overline{1}}=1$ and $\left.\bar{i}=i+g\right)$.

4.5. Specializations of the $t_{n, N}$ and examples for QRA's over QTQBA's. If $\mathfrak{g}$ is a Lie algebra over $\mathbf{k}, t_{\mathfrak{g}} \in S^{2}(\mathfrak{g})^{\mathfrak{g}}$, and $\sigma \in \operatorname{Aut}\left(\mathfrak{g}, t_{\mathfrak{g}}\right)$ is such that $\sigma^{N}=\mathrm{id}$, then $\left(\mathfrak{g}, t_{\mathfrak{g}}, \sigma\right)$ gives rise to a representation $T_{n, N} \rightarrow U(\mathfrak{l}) \otimes(U(\mathfrak{g}) \rtimes \mathbb{Z} / N \mathbb{Z})^{\otimes n}$ (here $\mathfrak{l}=\mathfrak{g}^{\sigma}$ ), as follows. Set $\mathfrak{u}=\operatorname{Im}(\sigma-\mathrm{id})$, then $\mathfrak{g}=\mathfrak{l} \oplus \mathfrak{u}$ and $t_{\mathfrak{g}}=t_{\mathfrak{l}} \oplus t_{\mathfrak{u}}$, where $t_{\mathfrak{l}} \in S^{2}(\mathfrak{l})$ and $t_{\mathfrak{u}} \in S^{2}(\mathfrak{u})$. The representation is $t_{0}^{0 i} \mapsto \hbar N\left(t_{\mathfrak{l}}^{0 i}+\frac{1}{2} t_{\mathfrak{l}}^{i i}\right), t(a)^{i j} \mapsto \hbar\left(\sigma^{a} \otimes \mathrm{id}\right)\left(t_{\mathfrak{g}}\right)^{i j}$, and $s_{i} \mapsto \underline{\sigma}^{i}$ (here $\hbar$ is a formal parameter and $\underline{\sigma}$ is the generator of $\mathbb{Z} / N \mathbb{Z} \subset U(\mathfrak{g}) \rtimes \mathbb{Z} / N \mathbb{Z})$. $\left(\mathfrak{g}, t_{\mathfrak{g}}, \sigma\right)$ also gives rise to a representation $\mathfrak{t}_{n} \rightarrow U(\mathfrak{g})^{\otimes n}, t^{i j} \mapsto \hbar t_{\mathfrak{g}}^{i j}$. This collection of representations is compatible with (a) the insertion-coproduct maps $U(\mathfrak{l}) \otimes(U(\mathfrak{g}) \rtimes \mathbb{Z} / N \mathbb{Z})^{\otimes n} \rightarrow U(\mathfrak{l}) \otimes(U(\mathfrak{g}) \rtimes \mathbb{Z} / N \mathbb{Z})^{\otimes m}$ induced by a partially defined function $f:[0, m] \rightarrow[0, n]$ with $f(0)=0$, and (b) the insertion-coproduct maps $U(\mathfrak{g})^{\otimes n} \rightarrow U(\mathfrak{l}) \otimes U(\mathfrak{g})^{\otimes m}$ induced by a partially defined function $f:[1, m] \rightarrow[1, n]$.

If $(a, \lambda, \Phi, \Psi) \in \underline{\operatorname{Pseudo}}(N, \mathbf{k})$, then $\left(U(\mathfrak{g}) \rtimes \mathbb{Z} / N \mathbb{Z}[[\hbar]], R=e^{\hbar(\lambda / 2) t_{\mathfrak{g}}}, \Phi\left(\hbar t_{\mathfrak{g}}^{12}, \hbar t_{\mathfrak{g}}^{23}\right)\right)$ is a QTQBA and

$$
\left(U(\mathfrak{l})[[\hbar]], E=e^{\hbar(\lambda / N)\left(t_{\mathfrak{l}}^{12}+\frac{1}{2} t_{\mathfrak{l}}^{22}\right)}\left(1 \otimes \underline{\sigma}^{a}\right), \Psi\left(\hbar\left(t_{\mathfrak{l}}^{12}+\frac{1}{2} t_{\mathfrak{l}}^{22}\right), \hbar t_{\mathfrak{g}}^{23}, \ldots, \hbar\left(1 \otimes \sigma^{N-1}\right)\left(t_{\mathfrak{g}}\right)^{23}\right)\right)
$$

is a QRA over it.

\section{The SEMIGRoups GTM AND $\underline{\widehat{\mathrm{GTM}}}$}

5.1. The semigroup GTM. Let $\left(A, \Delta_{A}, R, \Phi_{A}\right)$ be a QTQBA and let $\left(B, \Delta_{B}, E, \Psi_{B}\right)$ be a QRA over $A$. Let $P_{n} \subset B_{n}$ be the pure braid group. We have group morphisms $P_{n} \rightarrow\left(A^{\otimes n}\right)^{\times}$ and $P_{n} \rightarrow\left(B \otimes A^{\otimes n-1}\right)^{\times}$. Let us describe them explicitly when $n=3$.

We have an isomorphism $P_{3} \simeq P_{3}^{0} \times \mathbb{Z} \simeq F_{2} \times \mathbb{Z}$, where the center $\mathbb{Z}$ is generated by $\left(\sigma_{1} \sigma_{2}\right)^{3}=$ $\left(\sigma_{1}^{2} \sigma_{2}\right)^{2}=x_{12} x_{13} x_{23}\left(x_{12}=\sigma_{1}^{2}, x_{23}=\sigma_{2}^{2}, x_{13}=\sigma_{2} \sigma_{1}^{2} \sigma_{2}^{-1}\right)$ and $P_{3}^{0}=\operatorname{Ker}\left(P_{3} \rightarrow P_{2} \simeq \mathbb{Z}\right)$ where $P_{3} \rightarrow P_{2}$ is the erasing of the strand 2, i.e., $x_{12}, x_{23} \mapsto 0, x_{13} \mapsto 1 ; P_{3}^{0} \simeq F_{2}$ is freely generated by $x:=x_{12}$ and $y:=x_{23}$.

The morphism $\pi_{A}: P_{3} \rightarrow\left(A^{\otimes 3}\right)^{\times}$is given by

$$
\sigma_{1}^{2} \mapsto R^{2,1} R^{1,2}, \quad \sigma_{2}^{2} \mapsto \Phi_{A}^{-1} R^{3,2} R^{2,3} \Phi_{A}, \quad\left(\sigma_{1} \sigma_{2}\right)^{3} \mapsto R^{2,1} R^{1,2} \Phi_{A} R^{3,2}\left(\Phi_{A}^{1,3,2}\right)^{-1} R^{3,1} R^{1,3} \Phi_{A}^{1,3,2} R^{2,3} \Phi_{A}^{-1}
$$


The morphism $\pi_{B A}: P_{3} \rightarrow\left(B \otimes A^{\otimes 2}\right)^{\times}$is given by

$\sigma_{1}^{2} \mapsto E^{1,2}, \quad \sigma_{2}^{2} \mapsto \Psi_{B}^{-1} R^{3,2} R^{2,3} \Psi_{B}, \quad\left(\sigma_{1} \sigma_{2}\right)^{3} \mapsto E^{1,2} \Psi_{B}^{1,2,3} R^{3,2}\left(\Psi_{B}^{1,3,2}\right)^{-1} E^{1,3} \Psi_{B}^{1,3,2} R^{2,3}\left(\Psi_{B}^{1,2,3}\right)^{-1}$.

Let $\lambda \in 2 \mathbb{Z}+1, \mu \in \mathbb{Z}$ and $f:=f\left(\sigma_{1}^{2}, \sigma_{2}^{2}\right)\left(\sigma_{1} \sigma_{2}\right)^{3 a}, g:=g\left(\sigma_{1}^{2}, \sigma_{2}^{2}\right)\left(\sigma_{1} \sigma_{2}\right)^{3 a^{\prime}}$ be elements of $P_{3}$. Set

$$
\tilde{R}:=R\left(R^{2,1} R\right)^{(\lambda-1) / 2}, \quad \tilde{\Phi}_{A}:=\Phi_{A} \pi_{A}(f), \quad \tilde{E}:=E^{\mu}, \quad \tilde{\Psi}_{B}:=\Psi_{B} \pi_{B A}(g) .
$$

Let us write under which conditions $\left(A, \Delta_{A}, \tilde{R}, \tilde{\Phi}_{A}\right)$ is a QTQBA and $\left(B, \Delta_{B}, \tilde{E}, \tilde{\Psi}_{B}\right)$ is a QRA over it. According to [Dr2], $\left(A, \Delta_{A}, \tilde{R}, \tilde{\Phi}_{A}\right)$ is a QTQBA if $a=0$ and

$$
f(y, x)=f(x, y)^{-1}, \quad f\left(x_{3}, x_{1}\right) x_{3}^{(\lambda-1) / 2} f\left(x_{2}, x_{3}\right) x_{2}^{(\lambda-1) / 2} f\left(x_{1}, x_{2}\right) x_{1}^{(\lambda-1) / 2}=1
$$

where $x_{1}, x_{2}, x_{3}$ are three variables subject to the only relation $x_{1} x_{2} x_{3}=1$, and

$$
f\left(x_{12}, x_{23} x_{24}\right) f\left(x_{13} x_{23}, x_{34}\right)=f\left(x_{23}, x_{34}\right) f\left(x_{12} x_{13}, x_{24} x_{34}\right) f\left(x_{12}, x_{23}\right)
$$

holds in $P_{4}$, where $x_{i j}$ are the Artin generators

$$
x_{i j}=\left(\sigma_{j-2} \cdots \sigma_{i}\right)^{-1} \sigma_{j-1}^{2}\left(\sigma_{j-2} \cdots \sigma_{i}\right)=\left(\sigma_{j-1} \cdots \sigma_{i+1}\right) \sigma_{i}^{2}\left(\sigma_{j-1} \cdots \sigma_{i+1}\right)^{-1} \quad(1 \leq i<j \leq 4) .
$$

Lemma 5.1. If these conditions are met, $\left(B, \Delta_{B}, \tilde{E}, \tilde{\Psi}_{B}\right)$ is a $Q R A$ over $\left(A, \Delta_{A}, \tilde{R}, \tilde{\Phi}_{A}\right)$ if $a^{\prime}=0$,

$$
g(x, y)^{-1} y^{\frac{\lambda-1}{2}} g\left(x^{-1} y^{-1}, y\right)\left(x^{-1} y^{-1}\right)^{\mu} g\left(x^{-1} y^{-1}\right)^{-1} y^{\frac{\lambda+1}{2}} g(x, y) x^{\mu}=1
$$

(identity in the free group generated by $x, y$ ), and

$$
g\left(x_{01}, x_{12} x_{13}\right) g\left(x_{02} x_{12}, x_{23}\right)=f\left(x_{12}, x_{23}\right) g\left(x_{01} x_{02}, x_{13} x_{23}\right) g\left(x_{01}, x_{12}\right)
$$

holds in $P_{4}$ (with Artin generators $x_{i j}, 0 \leq i<j \leq 3$ ).

Proof. The identity implying that $\left(\tilde{\Phi}_{A}, \tilde{\Psi}_{B}\right)$ satisfies (13) is

$$
\begin{aligned}
& g\left(x_{12}, x_{23} x_{24}\right) z_{1,2,34}^{a^{\prime}} g\left(x_{13} x_{23}, x_{34}\right) z_{12,3,4}^{a^{\prime}} \\
& =f\left(x_{23}, x_{24}\right) g\left(x_{12} x_{13}, x_{24} x_{34}\right) z_{1,23,4}^{a^{\prime}} g\left(x_{12}, x_{13}\right) z_{1,2,3}^{a^{\prime}},
\end{aligned}
$$

where $z_{1,2,3}=\left(\sigma_{1} \sigma_{2}\right)^{3}=x_{23} x_{12} x_{13}, z_{2,3,4}=\left(\sigma_{2} \sigma_{3}\right)^{3}=x_{24} x_{34} x_{23}, z_{12,3,4}=x_{12}^{-1} z, x_{1,23,4}=$ $x_{23}^{-1} z, x_{1,2,34}=x_{34}^{-1} z$, where $z:=\left(\sigma_{3} \sigma_{2} \sigma_{1}\right)^{3}=x_{14} x_{24} x_{34} x_{13} x_{23} x_{12}$ generates the center of $P_{4}$. There is a unique morphism $P_{4} \rightarrow \mathbb{Z}$ with $x_{14} \mapsto 1, x_{i j} \mapsto 0$ for $1 \leq i<j \leq 4,(i, j) \neq(1,4)$ (it corresponds to the removal of strands 2,3$)$. The image of (20) by this morphism yields $2 a^{\prime}=a^{\prime}$, hence $a^{\prime}=0$. Then (20) implies (19). (18) is then the condition for $\left(\tilde{E}, \tilde{\Psi}_{B}\right)$ to satisfy (14).

Remark 5.2. (18) implies that $\theta_{(\lambda, \mu, g)} \in \operatorname{End}\left(F_{2}\right)$ ( $F_{2}$ is the free group generated by $x, y$ ) defined by $x \mapsto g(x, y) x^{\mu} g(x, y)^{-1}, y \mapsto y^{\lambda}$ also takes $x^{-1} y^{-1}$ to a conjugate of a power of this element, namely $x^{-1} y^{-1} \mapsto y^{\frac{\lambda-1}{2}} g\left(x^{-1} y^{-1}, y\right) \cdot\left(x^{-1} y^{-1}\right)^{\mu} \cdot\left(y^{\frac{\lambda-1}{2}} g\left(x^{-1} y^{-1}, y\right)\right)^{-1}$.

Define $\underline{\mathrm{GTM}}$ as the set of $(\lambda, \mu, f, g) \in(2 \mathbb{Z}+1) \times \mathbb{Z} \times F_{2} \times F_{2}$, satisfying (16), (17), (18) and (19). Then GTM has a semigroup structure, defined by

$$
\left(\lambda_{1}, \mu_{1}, f_{1}, g_{1}\right) *\left(\lambda_{2}, \mu_{2}, f_{2}, g_{2}\right)=(\lambda, \mu, f, g),
$$

where

$$
\begin{aligned}
& \lambda=\lambda_{1} \lambda_{2}, \quad f(x, y)=f_{1}\left(f_{2}(x, y) x^{\lambda_{2}} f_{2}(x, y)^{-1}, y^{\lambda_{2}}\right) f_{2}(x, y), \\
& \mu=\mu_{1} \mu_{2}, \quad g(x, y)=g_{1}\left(g_{2}(x, y) x^{\mu_{2}} g_{2}(x, y)^{-1}, y^{\lambda_{2}}\right) g_{2}(x, y) .
\end{aligned}
$$

Recall that $\underline{\text { GT }}$ was defined in [Dr2] as the semigroup of all pairs $(f, \lambda)$ satisfying (16), (17). 
We have a natural morphism $\underline{\mathrm{GTM}} \rightarrow \underline{\mathrm{GT}}$, taking $(\lambda, \mu, f, g)$ to $(\lambda, f)$. The semigroup $\underline{\mathrm{GTM}}$ acts on the set of pairs $(\mathcal{C}, \mathcal{M})$ of a braided monoidal category $\mathcal{C}$ and a braided module category $\mathcal{M}$ over $\mathcal{C}$ (GTM stands for "module category version of GT").

According to [Dr2], Proposition 4.1, GT consists of the pairs $(\lambda, f)$, where $\lambda= \pm 1$ and $f(x, y)=1 ;$ so $\underline{\mathrm{GT}} \simeq \mathbb{Z} / 2 \mathbb{Z}$, where $\mathbb{Z} / 2 \mathbb{Z}$ acts by $\left(A, \Delta_{A}, R, \Phi_{A}\right) \mapsto\left(A, \Delta_{A},\left(R^{2,1}\right)^{-1}, \Phi_{A}\right)$. In the same way, we have:

Proposition 5.3. GTM consists of the $(\lambda, \mu, f, g)$, where $\lambda=\mu= \pm 1, f(x, y)=1, g(x, y)=$ $y^{s}(s \in \mathbb{Z}$ ), so $\underline{\mathrm{GTM}} \simeq \mathbb{Z} \rtimes \mathbb{Z} / 2 \mathbb{Z}$ (the action of $\mathbb{Z} / 2 \mathbb{Z}$ on $\mathbb{Z}$ is nontrivial); $\mathbb{Z} / 2 \mathbb{Z}$ acts by $\left(A, \Delta_{A}, R, \Phi_{A} ; B, \Delta_{B}, E, \Psi_{B}\right) \mapsto\left(A, \Delta_{A},\left(R^{2,1}\right)^{-1}, \Phi_{A} ; B, \Delta_{B}, E^{-1}, \Psi_{B}\right)$.

Proof. We already have $\lambda= \pm 1$ and $f(x, y)=1$. Apply the morphism $P_{4} \rightarrow P_{3}, x_{i j} \mapsto x_{i j}$ if $i \geq 1, x_{0 j} \mapsto 1$ corresponding to removing the first strand, to (19). We get $g\left(x_{12}, x_{23}\right)=$ $\left(x_{12} x_{13}\right)^{-s} f\left(x_{12}, x_{23}\right)\left(x_{13} x_{23}\right)^{s} x_{12}^{s}$, where $s \in \mathbb{Z}$ is such that $g(1, y)=y^{s}$. Since $x_{12} x_{13} x_{23}$ is central in $P_{3}$, we get $g(x, y)=y^{s} f(x, y)=y^{s}$. Then $g(x, y)$ satisfies (19) and (18) is satisfied iff

$$
\left(x y x^{-1}\right)^{(\lambda-1) / 2}\left(y^{-1} x^{-1}\right)^{\mu-1} y^{(\lambda-1) / 2} x^{\mu-1}=1,
$$

which implies (as the degree in $y$ of the l.h.s. should vanish) $\lambda=\mu$, and since $\lambda= \pm 1$, we have necessarily $\lambda=\mu= \pm 1$. Conversely, (22) is satisfied when $\lambda=\mu= \pm 1$.

While the solutions of (16), (17), (18), (19) act on (general) braided module categories, solutions of these equations in completions of the pure braid groups act on braided module categories with additional properties.

5.2. The semigroup $\widehat{\text { GTM. }}$. If $G$ is a group (other that GT or GTM), we denote by $\widehat{G}$ (or $G^{\wedge}$ ) the profinite completion of $G$; if $\phi: G \rightarrow H$ is a group morphism, we denote by $\widehat{\phi}: \widehat{G} \rightarrow \widehat{H}$ the induced morphism.

Recall the definition of $\underline{\widehat{\mathrm{GT}}}([\mathrm{Dr} 2])$. The conditions expressing that $(\lambda, f) \in \underline{\mathrm{GT}}$ are expressed via group morphisms, namely $\kappa_{21}(f) f=1, \kappa_{31}(f) \kappa_{3}\left(\frac{\lambda-1}{2}\right) \kappa_{23}(f) \kappa_{2}\left(\frac{\lambda-1}{2}\right) f \kappa_{1}\left(\frac{\lambda-1}{2}\right)=1$, $\partial_{3}(f) \partial_{1}(f)=\partial_{0}(f) \partial_{2}(f) \partial_{4}(f)$, where $\kappa_{i j}: F_{2} \rightarrow F_{2}$ is $f\left(x_{1}, x_{2}\right) \rightarrow f\left(x_{i}, x_{j}\right), \kappa_{i}: \mathbb{Z} \rightarrow F_{2}$ is $\mu \mapsto y_{i}^{\mu}$, and $\partial_{i}: F_{2} \subset P_{3} \rightarrow P_{4}$ are the morphisms from [Dr2]; explicitly, $\partial_{0}: x, y \mapsto x_{12}, x_{23}$, $\partial_{4}: x, y \mapsto x_{01}, x_{12}, \partial_{1}: x, y \mapsto x_{02} x_{12}, x_{23}$, and $\partial_{2}: x, y \mapsto x_{01} x_{02}, x_{13} x_{23}$. On the other hand, the composition is $f:=\theta_{\left(\lambda_{2}, f_{2}\right)}\left(f_{1}\right) f_{2}$, where $\theta_{(\lambda, f)}: F_{2} \rightarrow F_{2}$ is $(x, y) \mapsto\left(f x^{\lambda} f^{-1}, y^{\lambda}\right)$. $\widehat{\text { GT }}$ is then defined as the set of $(\lambda, f) \in(2 \widehat{\mathbb{Z}}+1) \times \widehat{F}_{2}$, satisfying the profinite analogues of the above conditions. Any $(\lambda, f) \in \widehat{\mathbb{Z}} \times \widehat{F}_{2}$ gives rise to $\theta_{(\lambda, f)} \in \operatorname{End}\left(\widehat{F}_{2}\right)$, which serves to define the composition in $\widehat{\mathrm{GT}}$.

In the same way, we define $\underline{\widehat{\mathrm{GTM}}}$ as the set of all $(\lambda, \mu, f, g) \in(2 \widehat{\mathbb{Z}}+1) \times \widehat{\mathbb{Z}} \times \widehat{F}_{2} \times \widehat{F}_{2}$ satisfying the profinite analogues of the defining conditions of GTM. The composition formulas for GTM extend to $\widehat{\widehat{G T M}}$ and define a semigroup structure. $\widehat{\widehat{G T M}}$ acts on the pairs $(\mathcal{C}, \mathcal{M})$, such that the image of the braid groups are finite. We have a semigroup morphism $\underline{\widehat{\mathrm{GTM}}} \rightarrow \underline{\widehat{\mathrm{GT}}}$, compatible with the action of $\widehat{\underline{\mathrm{GT}}}$ on similar categories $\mathcal{C}$.

Proposition 5.4. $\underline{\widehat{\mathrm{GTM}}}=\left\{\left(\lambda, \lambda, f, y^{s} f\right) \mid(\lambda, f) \in \underline{\widehat{\mathrm{GT}}}\right.$ and $\left.s \in \widehat{\widehat{\mathbb{Z}}}\right\}$. So $\underline{\widehat{\mathrm{GTM}}} \simeq \underline{\widehat{\mathrm{GT}}} \times \widehat{\mathbb{Z}}$, where $\widehat{\mathbb{Z}}$ is equipped with its additive structure and the action of $\widehat{\mathrm{GT}}$ on it is via the semigroup character $\chi: \underline{\widehat{G T}} \rightarrow \widehat{\mathbb{Z}},(f, \lambda) \mapsto \lambda$ (where $\widehat{\mathbb{Z}}$ is equipped with its mutiplicative semigroup structure).

Proof. Let $(f, \lambda) \in \underline{\widehat{G T}}$ and let us find the set of all $(g, \mu)$ such that $(\lambda, \mu, f, g) \in \widehat{\text { GTM}}$. Let

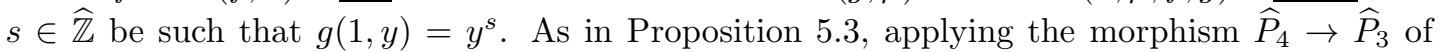
removal of the first strand to (19), we get $g(x, y)=y^{s} f(x, y)$. Abelianizing the profinite version of (18), we find that $\mu=\lambda$. Conversely, one shows that for any $s \in \widehat{\mathbb{Z}},\left(\lambda, \lambda, f, y^{s} f\right) \in \widehat{\mathrm{GTM}}$. 
So we have a bijection $\underline{\widehat{G T M}} \simeq \underline{\widehat{G T}} \times \widehat{\mathbb{Z}}$. One then checks that the semigroup structure is the semidirect product $\left(\underline{f}_{1}, s_{1}\right)\left(\underline{f}_{2}, s_{2}\right)=\left(\underline{f}_{1} \underline{f}_{2}, s_{1} \chi\left(\underline{f}_{2}\right)+s_{2}\right)$.

We now identify $\left(\lambda, \lambda, f, y^{s} f\right) \in \underline{\widehat{\mathrm{GTM}}}$ with $((f, \lambda), s) \in \underline{\widehat{\mathrm{GT}}} \times \widehat{\mathbb{Z}}$. If $G$ is a semigroup, we denote by $G^{i n v}$ the group of its invertible elements. Recall that $\widehat{\widehat{G T}}^{i n v}$ is denoted $\widehat{\mathrm{GT}}$ and that $\widehat{\mathrm{GT}} \subset \underline{\widehat{\mathrm{GT}}} \times \widehat{\mathbb{Z}}^{\times}$. If we set $\widehat{\mathrm{GTM}}:=\widehat{\widehat{\mathrm{GTM}}}^{i n v}$, then $\widehat{\mathrm{GTM}}=\widehat{\mathrm{GT}} \times \widehat{\mathbb{Z}}=\{((f, \lambda), s) \mid(f, \lambda) \in \widehat{\mathrm{GT}}\}$.

\section{The Groups $\operatorname{GTM}(N)_{l} \operatorname{AND} \operatorname{GTM}(N, \mathbf{k})$}

If $G$ is a group and $l$ is a prime number, we denote by $G_{l}$ its pro- $l$ completion.

6.1. Partial profinite and pro- $l$ completions. Let $\varphi: \Gamma \rightarrow \Gamma_{0}$ be a surjective group morphism. There exists a group $\Gamma(\varphi, l)$, fitting in an exact sequence $1 \rightarrow\left(\Gamma_{1}\right)_{l} \rightarrow \Gamma(\varphi, l) \rightarrow$ $\Gamma_{0} \rightarrow 1$, and a group morphism $\Gamma \rightarrow \Gamma(\varphi, l)$, such that the diagram

$$
\begin{aligned}
& 1 \rightarrow \Gamma_{1} \quad \rightarrow \quad \Gamma \quad \rightarrow \quad \Gamma_{0} \rightarrow 1
\end{aligned}
$$

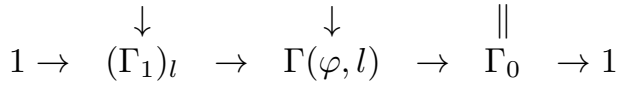

commutes and with universal properties similar to those of partial prounipotent completions (see Section 1.1).

We also have a group $\widehat{\Gamma}(\varphi)$ with the same properties, where $\left(\Gamma_{1}\right)_{l}$ is replaced by $\widehat{\Gamma}_{1}$; we then have a morphism $\widehat{\Gamma}(\varphi) \rightarrow \Gamma(\varphi, l)$, fitting in a commutative diagram $\Gamma$

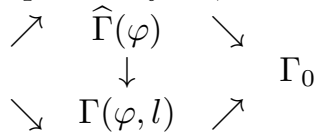

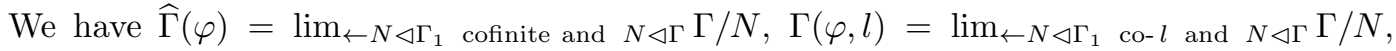
where " $N \triangleleft \Gamma_{1}$ cofinite" (resp., co-l) means that $\Gamma_{1} / N$ is finite (resp., a l-group).

By universal properties, the construction of $\Gamma(\varphi, l)$ and $\widehat{\Gamma}(\varphi)$ is functorial w.r.t. commuting $\begin{array}{rlc}\Gamma & \rightarrow & \Gamma_{0} \\ \text { squares } \downarrow & & \downarrow \\ \Gamma^{\prime} & \rightarrow & \Gamma_{0}^{\prime}\end{array}$

Lemma 6.1. 1) If $\varphi: \Gamma \rightarrow \Gamma_{0}$ and $\pi: \Gamma_{0} \rightarrow \Gamma_{0}^{\prime}$ are surjective group morphisms, such that $\operatorname{Ker} \pi$ is a l-group, then we have an isomorphism $\Gamma(\varphi, l) \simeq \Gamma(\pi \circ \varphi, l)$, such that the diagram $\Gamma(\varphi, l) \simeq \Gamma(\pi \circ \varphi, l)$

$\downarrow \quad \downarrow \quad$ commutes.

$\Gamma_{0} \quad \stackrel{\pi}{\rightarrow} \quad \Gamma_{0}^{\prime}$

2) Same statements replacing "l-group" by "finite" and $\Gamma(\psi, l)$ by $\widehat{\Gamma}(\psi)(\psi=\varphi, \pi \circ \varphi)$.

Proof. Let us prove 1) (2) is proved in the same way). By universal properties, we have a morphism $\Gamma(\varphi, l) \rightarrow \Gamma(\pi \circ \varphi, l)$ commuting with the inclusions of $\Gamma$ and the morphisms to $\Gamma_{0}^{\prime}$. To show that this is an isomorphism, reinterpret this map as follows. We have $\Gamma(\varphi, l)=$ $\lim _{\leftarrow N \in \mathcal{S}} \Gamma / N$ and $\Gamma(\pi \circ \varphi, l)=\lim _{\leftarrow N^{\prime} \in \mathcal{S}^{\prime}} \Gamma / N^{\prime}$, where $\mathcal{S}=\left\{N \mid N \triangleleft \Gamma_{1}\right.$ co-l, $\left.N \triangleleft \Gamma\right\}, \mathcal{S}^{\prime}=$ $\left\{N^{\prime} \mid N^{\prime} \triangleleft \Gamma_{1}^{\prime} \operatorname{co}-l, N^{\prime} \triangleleft \Gamma\right\}$ (recall that $\Gamma_{1}=\operatorname{Ker} \varphi$; we set $\Gamma_{1}^{\prime}:=\operatorname{Ker} \pi \circ \varphi$ ). The morphism is then induced by the map $\mathcal{S}^{\prime} \rightarrow \mathcal{S}, N^{\prime} \mapsto N^{\prime}$ (which is contained in $N^{\prime}$ ). A morphism $\Gamma(\pi \circ \varphi, l) \rightarrow \Gamma(\varphi, l)$ is then induced by $\mathcal{S} \rightarrow \mathcal{S}^{\prime}, N \mapsto \cap_{\gamma \in \Gamma_{1} / \Gamma_{1}^{\prime}} \tilde{\gamma} N^{-1}$ (which is contained in $N)$, where $\gamma \mapsto \tilde{\gamma}$ is a section of the projection $\Gamma_{1} \rightarrow \Gamma_{1} / \Gamma_{1}^{\prime}$.

If $\widehat{\Gamma}$ is the profinite completion of $\Gamma$ and $\Gamma_{0}$ is finite, then we have a commuting triangle $\widehat{\Gamma} \rightarrow \Gamma(\varphi, l)$

$\searrow \quad \swarrow$. The morphism $\widehat{\Gamma} \rightarrow \Gamma(\varphi, l)$ is the composition $\widehat{\Gamma} \simeq \widehat{\Gamma}(\varphi) \rightarrow \Gamma(\varphi, l)$. 
Recall that if $F_{n}$ is the free group with $n$ generators, we have an injection $\left(F_{n}\right)_{l} \hookrightarrow F_{n}\left(\mathbb{Q}_{l}\right)$. This implies that if $G$ is finitely generated, we have a functorial morphism $G_{l} \rightarrow G\left(\mathbb{Q}_{l}\right)$. More generally, if $\Gamma_{1}$ is finitely generated, then one has a morphism $\Gamma(\varphi, l) \rightarrow \Gamma\left(\varphi, \mathbb{Q}_{l}\right)$, and a com-

$\begin{array}{rrrrrr}\Gamma & \stackrel{\varphi}{\rightarrow} & \Gamma_{0} & \Gamma(\varphi, l) & \rightarrow & \Gamma\left(\varphi, \mathbb{Q}_{l}\right) \\ \text { muting square } f \downarrow & & \downarrow \text { gives rise to a commuting square } f\left(\varphi, \varphi^{\prime}, l\right) \downarrow & & \downarrow \\ \Gamma^{\prime} & \stackrel{\varphi^{\prime}}{\rightarrow} & \Gamma_{0}^{\prime} & \Gamma^{\prime}\left(\varphi^{\prime}, l\right) & \rightarrow & \Gamma^{\prime}\left(\varphi^{\prime}, \mathbb{Q}_{l}\right)\end{array}$.

6.2. The rings $R(N)$. Let $R$ be a ring with unit. We attach to it a ring $R(N)$, equipped with a surjective ring morphism $R(N) \rightarrow \mathbb{Z} / N \mathbb{Z}$ and an exact sequence $0 \rightarrow R \rightarrow R(N) \rightarrow \mathbb{Z} / N \mathbb{Z} \rightarrow 0$ of additive groups.

The ring $R(N)$ is defined as follows: $R(N)=(\mathbb{Z} / N \mathbb{Z}) \times R$, with addition defined by $(a, r)+$ $\left(a^{\prime}, r^{\prime}\right)=\left(a+a^{\prime}, r+r^{\prime}+\sigma\left(a, a^{\prime}\right) N\right)$, where $\sigma\left(a, a^{\prime}\right) \in\{0,1\}$ is defined by $\tilde{a}+\tilde{a}^{\prime}=\widetilde{a+a^{\prime}}+\sigma\left(a, a^{\prime}\right) N$ (we denote by $\tilde{x} \in[0, N-1]$ the representative of $x \in \mathbb{Z} / N \mathbb{Z}$ ). The product is defined by $(a, r)\left(a^{\prime}, r^{\prime}\right)=\left(a a^{\prime}, r a^{\prime}+a r^{\prime}+N r r^{\prime}+\pi\left(a, a^{\prime}\right) N\right)$, where $\pi\left(a, a^{\prime}\right) \in \mathbb{N}$ is defined by $\tilde{a} \tilde{a}^{\prime}=$ $\widetilde{a a^{\prime}}+\pi\left(a, a^{\prime}\right) N$.

The assignment $R \mapsto R(N)$ is an endofunctor of the category of rings with unit with the following properties:

(a) if $N_{1}$ and $N_{2}$ are coprime, then $R\left(N_{1}\right)\left(N_{2}\right) \simeq R\left(N_{1} N_{2}\right)$;

(b) we have a ring morphism $R(N) \rightarrow(\mathbb{Z} / N \mathbb{Z}) \times R, \lambda \mapsto(\bar{\lambda},[\lambda])$, where $\bar{\lambda}=a$ and $[\lambda]=\tilde{a}+N r$ for $\lambda=(a, r)$; this is an isomorphism if $N$ is invertible in $R$;

(b') more generally, if $N^{\prime} \mid N$ and $d=N / N^{\prime}$, we have a ring morphism [[-]]:R(N) $\rightarrow R\left(N^{\prime}\right)$, $(a, r) \mapsto(\overline{\bar{a}}, \tilde{\tilde{a}}+d r)$, where $a \mapsto \overline{\bar{a}}$ is the morphism $\mathbb{Z} / N \mathbb{Z} \rightarrow \mathbb{Z} / d \mathbb{Z}, \overline{1} \mapsto \overline{1} ; \tilde{\tilde{a}} \in\left[0, N^{\prime}-1\right]$ is the integral part of $\tilde{a} / d$; if $d$ is invertible in $R$, then $R(N) \rightarrow(\mathbb{Z} / d \mathbb{Z}) \times R\left(N^{\prime}\right),(a, r) \mapsto(\bar{a},[[(a, r)]])$ is an isomorphism (where $a \mapsto \bar{a}$ is $\mathbb{Z} / N \mathbb{Z} \rightarrow \mathbb{Z} / d \mathbb{Z}, \overline{1} \mapsto \overline{1}$ );

(c) if $R, R^{\prime}$ are rings with unit, then $\left(R \times R^{\prime}\right)(N)=R(N) \times_{\mathbb{Z} / N \mathbb{Z}} R^{\prime}(N)$.

If $l$ is a prime number, then $\left(\mathbb{Z} / l^{n} \mathbb{Z}\right)(l) \simeq \mathbb{Z} / l^{n+1} \mathbb{Z}$; it follows that $\mathbb{Z}_{l}(l) \simeq \mathbb{Z}_{l}$. Using (a) and (b), we then get $\mathbb{Z}_{l}(N) \simeq \mathbb{Z}_{l} \times\left(\mathbb{Z} / N^{\prime} \mathbb{Z}\right)$, where $N^{\prime}=N / l^{\alpha}$ and $\alpha$ is the $l$-adic valuation of $N$. (c) then implies that $\widehat{\mathbb{Z}}(N) \simeq \widehat{\mathbb{Z}}$. One also checks directly that the map $(a, r) \mapsto \tilde{a}+N r$ induces an isomorphism $\mathbb{Z}(N) \simeq \mathbb{Z}$.

If $l$ is a prime number, then $\mathbb{Z}($ can,$l) \simeq \mathbb{Z}_{l}(N)$, where can : $\mathbb{Z} \rightarrow \mathbb{Z} / N \mathbb{Z}$ is the canonical projection; on the other hand, $\mathbb{Z}(0, l)=\mathbb{Z}_{l}$. If $N^{\prime} \mid N$, one checks that the morphism $\mathbb{Z}(N)_{l} \rightarrow$

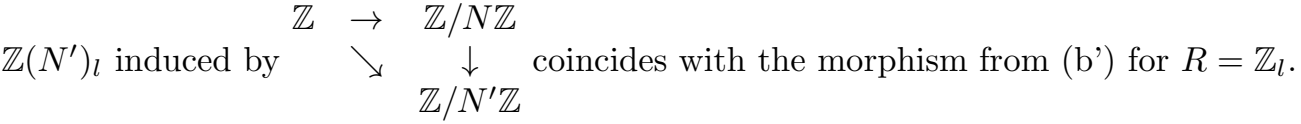

6.3. The semigroup $\underline{\operatorname{GTM}}(N)_{l}$. Recall that $\underline{\mathrm{GT}}_{l}$ is the set of pairs $(\lambda, f) \in\left(2 \mathbb{Z}_{l}+1\right) \times\left(F_{2}\right)_{l}$, satisfying the pro- $l$ versions of the defining conditions of GT; the duality and hexagon relations take place in $\left(F_{2}\right)_{l}$ and the pentagon relation in $\left(P_{4}\right)_{l}$. We then have a semigroup morphism $\widehat{\mathrm{GT}} \rightarrow \underline{\mathrm{GT}_{l}} ; \underline{\mathrm{GT}_{l}}$ acts on the braided monoidal categories $\mathcal{C}$, such that the image of the pure braid groups are $l$-groups.

We now introduce a semigroup $\underline{\operatorname{GTM}}(N)_{l}$ acting on pairs $(\mathcal{C}, \mathcal{M})$, such that the images of $K_{n, N} \subset B_{n}^{1}$ are l-groups. The setup of the definition of GTM has to be modified as follows: $\lambda \in 2 \mathbb{Z}_{l}+1, f \in\left(P_{3}^{0}\right)_{l}=\left(F_{2}\right)_{l} ; \mu \in \mathbb{Z}(\operatorname{can}, l)=\mathbb{Z}_{l}(N)$, and $g \in P_{3}^{0}\left(\varphi_{3, N}, l\right) \simeq F_{2}\left(\varphi_{N}, l\right) ;$ here $^{5}$ $\varphi_{N}: F_{2} \rightarrow \mathbb{Z} / N \mathbb{Z}$ is defined by $x \mapsto \overline{1}, y \mapsto \overline{0}$.

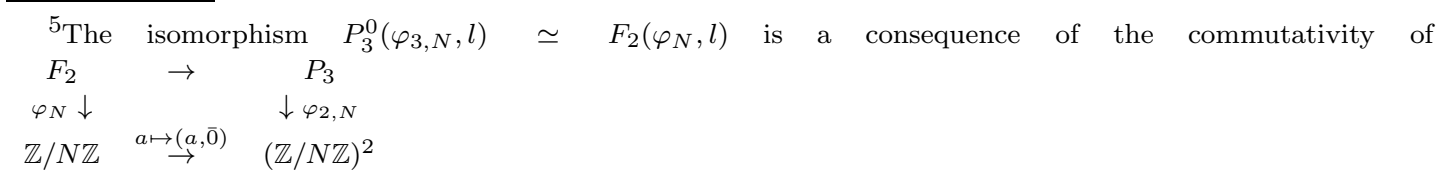


We now describe the conditions defining $\underline{\operatorname{GTM}}(N)_{l}$. Recall that the octogon condition (18) can be written

$$
g^{-1} \kappa_{y}\left(\frac{\lambda-1}{2}\right) \kappa(g) \kappa_{z}(\mu) \kappa(g)^{-1} \kappa_{y}\left(\frac{\lambda+1}{2}\right) g \kappa_{x}(\mu)=1,
$$

where the morphisms $\kappa_{x}, \kappa_{y}, \kappa_{z}: \mathbb{Z} \rightarrow F_{2}$ are $a \mapsto x^{a}, a \mapsto y^{a}, a \mapsto\left(y^{-1} x^{-1}\right)^{a}$, and $\kappa: F_{2} \rightarrow F_{2}$ is $g(x, y) \mapsto g\left(x^{-1} y^{-1}, y\right)$.

Now $\varphi_{N} \circ \kappa_{y}=0$, so $\kappa_{y}$ extends to a group morphism $\mathbb{Z}_{l} \rightarrow F_{2}\left(\varphi_{N}, l\right)$, and the dia$\mathbb{Z} \stackrel{\kappa_{x} z}{\rightarrow} \quad F_{2}$

grams can $\downarrow \quad \downarrow \varphi_{N}$ commute, which implies that $\kappa_{x, z}$ extend to a morphism $\mathbb{Z}_{l}(N) \rightarrow$ $\mathbb{Z} / N \mathbb{Z} \stackrel{ \pm \text { id }}{\rightarrow} \mathbb{Z} / N \mathbb{Z}$

$F_{2}\left(\varphi_{N}, l\right)$. We also have a commuting diagram $\begin{array}{ccc}F_{2} & \stackrel{\kappa}{\rightarrow} \varphi_{N} \downarrow & F_{2} \\ & \downarrow \varphi_{N} & \text {, so } \kappa \text { extends to an en- }\end{array}$ $\mathbb{Z} / N \mathbb{Z} \stackrel{- \text { id }}{\rightarrow} \mathbb{Z} / N \mathbb{Z}$

domorphism of $F_{2}\left(\varphi_{N}, l\right)$. All this implies that $(\lambda, \mu, g) \mapsto$ l.h.s. of (18) extends to a map $\operatorname{oct}_{N, l}:\left(2 \mathbb{Z}_{l}+1\right) \times \mathbb{Z}_{l}(N) \times F_{2}\left(\varphi_{N}, l\right) \rightarrow F_{2}\left(\varphi_{N}, l\right)$.

The mixed pentagon condition (19) is written

$$
\partial_{3}(g) \partial_{1}(g)=\partial_{0}(f) \partial_{2}(g) \partial_{4}(g)
$$

For $i=1, \ldots, 4$, we have commuting diagrams $\begin{array}{ccc}F_{2} & \stackrel{\partial_{3}}{\rightarrow} & P_{4} \\ \varphi_{N} \downarrow & & \downarrow \varphi_{3, N} \\ \mathbb{Z} / N \mathbb{Z} & \stackrel{\alpha_{i}}{\rightarrow} & (\mathbb{Z} / N \mathbb{Z})^{3}\end{array}$ where $\alpha_{i}(\overline{1})=(\overline{0}, \overline{1}, \overline{0})$ for $i=1,(\overline{1}, \overline{1}, \overline{0})$ for $i=2$, $(\overline{1}, \overline{0}, \overline{0})$ for $i=3$, 4; moreover, $\varphi_{3, N} \circ \partial_{0}=0$. So the morphisms $\partial_{i}: F_{2} \rightarrow P_{4}$ induce, for $i=1, \ldots, 4$, group morphisms $F_{2}\left(\varphi_{N}, l\right) \rightarrow P_{4}\left(\varphi_{3, N}, l\right)$, and the morphism $\partial_{0}: F_{2} \rightarrow P_{4}$ induces a morphism $\left(F_{2}\right)_{l} \rightarrow P_{4}\left(\varphi_{3, N}, l\right)$. It follows that the map $(f, g) \mapsto$ l.h.s. of (19) extends to a map pent ${ }_{N, l}:\left(F_{2}\right)_{l} \times F_{2}\left(\varphi_{N}, l\right) \rightarrow P_{4}\left(\varphi_{3, N}, l\right)$.

Definition 6.2. $\underline{\operatorname{GTM}}(N)_{l}$ is the set of all $(\lambda, \mu, f, g) \in\left(2 \mathbb{Z}_{l}+1\right) \times \mathbb{Z}_{l}(N) \times\left(F_{2}\right)_{l} \times F_{2}\left(\varphi_{N}, l\right)$, such that $(\lambda, f) \in \underline{\mathrm{GT}}_{l}, \operatorname{oct}_{N, l}(\lambda, \mu, g)=1$ and $\operatorname{pent}_{N, l}(f, g)=1$.

We now define the composition in $\underline{\operatorname{GTM}}(N)_{l}$.

We define $\lambda \mapsto x^{\lambda}, y^{\lambda}$ as the morphisms $\mathbb{Z}_{l} \rightarrow\left(F_{2}\right)_{l}$ induced by $\mathbb{Z} \rightarrow F_{2}, a \mapsto x^{a}, y^{a}$. Then for $(\lambda, f) \in \mathbb{Z}_{l} \times\left(F_{2}\right)_{l}$, there is a unique $\theta_{(\lambda, f)} \in \operatorname{End}\left(\left(F_{2}\right)_{l}\right)$ given by $x \mapsto f x^{\lambda} f^{-1}, y \mapsto y^{\lambda}$.

Define $\mu \mapsto x^{\mu}$ as the morphism $\mathbb{Z}_{l}(N) \rightarrow F_{2}\left(\varphi_{N}, l\right)$ induced by $\mathbb{Z} \rightarrow F_{2}, a \mapsto x^{a}$ and $\lambda \mapsto y^{\lambda}$ as the morphism $\mathbb{Z}_{l} \rightarrow F_{2}\left(\varphi_{N}, l\right)$ induced by $\mathbb{Z} \rightarrow F_{2}, a \mapsto y^{a}$. For $(\lambda, \mu, g) \in$ $\mathbb{Z}_{l} \times \mathbb{Z}_{l}(N) \times F_{2}\left(\varphi_{N}, l\right)$, there is a unique $\theta_{(\lambda, \mu, g)} \in \operatorname{End}\left(F_{2}\left(\varphi_{N}, l\right)\right)$ given by $x \mapsto g x^{\mu} g^{-1}$, $y \mapsto y^{\lambda}$.

The composition law of $\operatorname{GTM}(N)_{l}$ is defined by the same formulas as for GTM, where the composition for $f$ is understood as $f:=\theta_{\left(\lambda_{2}, f_{2}\right)}\left(f_{1}\right) f_{2}$ and the composition for $g$ as $g:=\theta_{\left(\lambda_{2}, \mu_{2}, g_{2}\right)}\left(g_{1}\right) g_{2}$. One then checks that $\underline{\operatorname{GTM}}(N)_{l}$ is a semigroup and $\underline{\operatorname{GTM}}(N)_{l} \rightarrow \underline{\mathrm{GT}_{l}}$, $(\lambda, \mu, f, g) \mapsto(\lambda, f)$ is a semigroup morphism.

Lemma 6.3. If $(\lambda, \mu, f, g) \in \underline{\operatorname{GTM}}(N)_{l}$, then $\lambda=[\mu]$, where $[-]$ is the morphism $\mathbb{Z}_{l}(N) \rightarrow \mathbb{Z}_{l}$ from 6.2, b).

$$
\begin{aligned}
F_{2} & \rightarrow \quad \mathbb{Z}^{2} \\
\text { Proof. The abelianization morphism } F_{2} \rightarrow \mathbb{Z}^{2} \text { fits in a commutative diagram } \varphi_{N} \downarrow & \begin{array}{r}
(a, b) \mapsto \\
\operatorname{can}(a)
\end{array} \\
\mathbb{Z} / N \mathbb{Z} & \stackrel{\text { id }}{\rightarrow} \mathbb{Z} / N \mathbb{Z}
\end{aligned}
$$

so it induces a morphism ab : $F_{2}\left(\varphi_{N}, l\right) \rightarrow \mathbb{Z}_{l}(N) \times \mathbb{Z}_{l}$. Let us apply ab to (23). We have $\operatorname{ab}\left(\kappa_{y}(\nu)\right)=(0, \nu)$ for $\nu \in \mathbb{Z}_{l}$, and for $\nu^{\prime} \in \mathbb{Z}_{l}(N), \operatorname{ab}\left(\kappa_{x}\left(\nu^{\prime}\right)\right)=\left(\nu^{\prime}, 0\right)$ and $\operatorname{ab}\left(\kappa_{z}\left(\nu^{\prime}\right)\right)=$ 
$-\left(\nu^{\prime},\left[\nu^{\prime}\right]\right)$; this follows from the fact that the morphism $\mathbb{Z}_{l}(N) \rightarrow \mathbb{Z}_{l}$ induced by the diagram

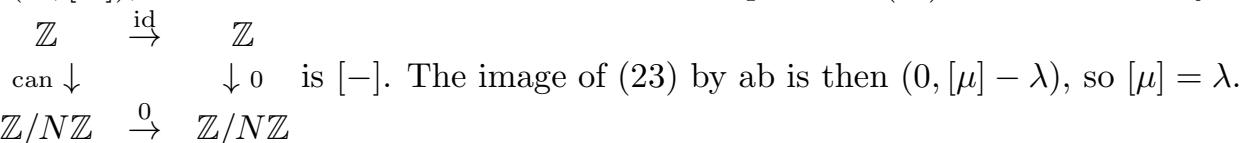

Let $b: F_{2} \rightarrow \mathbb{Z}$ be the morphism $x \mapsto 1, y \mapsto 0$. We have a commutative diagram $F_{2} \stackrel{b}{\rightarrow} \mathbb{Z}$

$\varphi_{N} \downarrow \quad \downarrow$ can hence $b$ induces a morphism $b_{l}: F_{2}\left(\varphi_{N}, l\right) \rightarrow \mathbb{Z}_{l}(N)$. In particular, the $\mathbb{Z} / N \mathbb{Z} \stackrel{\text { id }}{\rightarrow} \mathbb{Z} / N \mathbb{Z}$

morphism $F_{2}\left(\varphi_{N}, l\right) \stackrel{\left(\varphi_{N}\right)_{l}}{\rightarrow} \mathbb{Z} / N \mathbb{Z}$ induced by $\varphi_{N}$ factors as $F_{2}\left(\varphi_{N}, l\right) \stackrel{b_{l}}{\rightarrow} \mathbb{Z}_{l}(N) \rightarrow \mathbb{Z} / N \mathbb{Z}$.

Lemma 6.4. If $(\lambda, \mu, f, g) \in \underline{\operatorname{GTM}}(N)_{l}$, then $g \in \operatorname{Ker}\left[b_{l}: F_{2}\left(\varphi_{N}, l\right) \rightarrow \mathbb{Z}_{l}(N)\right]$; in particular, $g \in \operatorname{Ker}\left(\varphi_{N}\right)_{l}=\left(\operatorname{Ker} \varphi_{N}\right)_{l}$.

Proof. It is well-known that $f$ belongs to the kernel of the abelianization morphism $\left(F_{2}\right)_{l} \rightarrow$ $\mathbb{Z}_{l}^{\oplus 2}$ (this can be checked by abelianizing the pentagon identity). The mixed pentagon identity can be similarly abelianized: the abelianizations of the maps $\partial_{i}(i=0, \ldots, 4)$ fit in commuting

$$
\begin{aligned}
& F_{2} \quad \stackrel{\partial_{i}}{\rightarrow} \quad P_{4} \\
& \text { squares } \quad \mathrm{ab} \downarrow \quad \downarrow \mathrm{ab} \quad \text { which give rise to squares } \\
& \mathbb{Z} x^{a b} \oplus \mathbb{Z} y^{a b} \stackrel{\partial_{i}^{a b}}{\rightarrow} \oplus_{(i, j) \mid 0 \leq i<j \leq 3} \mathbb{Z} x_{i j}^{a b}
\end{aligned}
$$

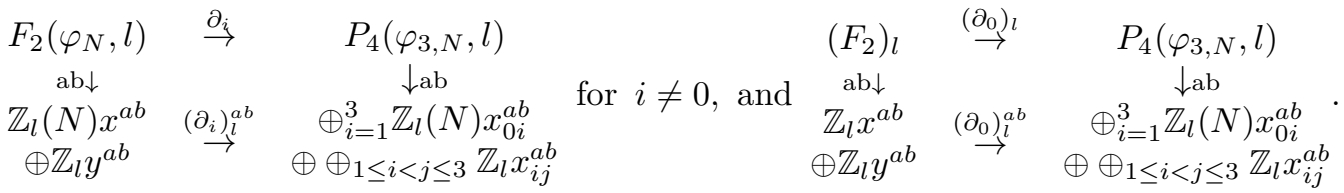

Set $\operatorname{ab}(g)=b x^{a b}+c y^{a b}, b \in \mathbb{Z}_{l}(N), c \in \mathbb{Z}_{l}$; in fact, $b=b_{l}(g)$. Then $\left(\partial_{2}\right)_{l}(g) \stackrel{\text { ab }}{\mapsto} b\left(x_{01}^{a b}+x_{02}^{a b}\right)+$ $c\left(x_{13}^{a b}+x_{23}^{a b}\right),\left(\partial_{4}\right)_{l}(g) \stackrel{\text { ab }}{\mapsto} b x_{01}^{a b}+c x_{12}^{a b},\left(\partial_{3}\right)_{l}(g) \stackrel{\text { ab }}{\mapsto} b x_{01}^{a b}+c\left(x_{12}+x_{13}\right)^{a b}$, and $\left(\partial_{1}\right)_{l}(g) \stackrel{\text { ab }}{\mapsto} b x_{02}^{a b}+$ $[b] x_{12}^{a b}+c x_{23}^{a b}$. Since $\left(\partial_{0}\right)_{l}(f) \stackrel{\text { ab }}{\mapsto} 0$, we get $\mathrm{ab}\left(\left(\partial_{2}\right)_{l}(g)\right)+\mathrm{ab}\left(\left(\partial_{4}\right)_{l}(g)\right)=\mathrm{ab}\left(\left(\partial_{1}\right)_{l}(g)\right)+\mathrm{ab}\left(\left(\partial_{3}\right)_{l}(g)\right)$, which implies $b=0$, as wanted.

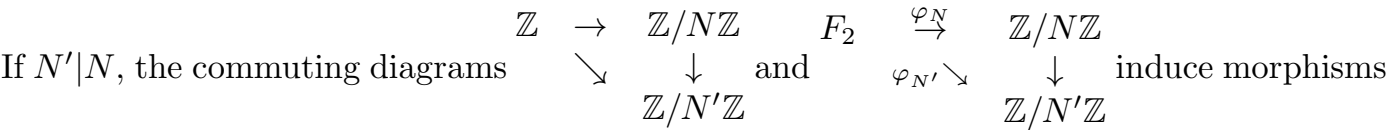
$\pi_{N N^{\prime}}: \mathbb{Z}_{l}(N) \rightarrow \mathbb{Z}_{l}\left(N^{\prime}\right), F_{2}\left(\varphi_{N}, l\right) \rightarrow F_{2}\left(\varphi_{N^{\prime}}, l\right)$ which take place in commuting squares $F_{2}\left(\varphi_{N}, l\right) \rightarrow F_{2}\left(\varphi_{N^{\prime}}, l\right)$

$\partial_{1,2,3} \downarrow \quad \downarrow \partial_{1,2,3}$, etc. We derive from there morphisms

$P_{4}\left(\varphi_{N}, l\right) \rightarrow P_{4}\left(\varphi_{N^{\prime}}, l\right)$

$$
\pi_{N N^{\prime}}: \underline{\operatorname{GTM}}(N)_{l} \rightarrow \underline{\operatorname{GTM}}\left(N^{\prime}\right)_{l},
$$

such that $\pi_{N N^{\prime \prime}}=\pi_{N^{\prime} N^{\prime \prime}} \circ \pi_{N N^{\prime}}$. The categorical interpretation of $\pi_{N N^{\prime}}$ is that as $K_{n, N} \subset$ $K_{n, N^{\prime}}$, we have $\operatorname{Cat}_{N^{\prime}} \subset \operatorname{Cat}_{N}$ where $\operatorname{Cat}_{N}=\left\{\right.$ pairs $(\mathcal{C}, \mathcal{M})$ where the image of $K_{n, N}$ in each $\operatorname{Aut}_{\mathcal{M}}\left(M \otimes X^{\otimes n}\right)$ is a l-group $\}$; the action of $g \in \underline{\operatorname{GTM}}(N)_{l}$ restricts to the action of $\pi_{N N^{\prime}}(g)$ on $\mathrm{Cat}_{N^{\prime}}$.

If now $N^{\prime}=N / l^{\alpha}$, where $\alpha$ is the $l$-adic valuation of $N, \pi_{N N^{\prime}}$ is an isomorphism as $\mathbb{Z}_{l}(N) \simeq$ $\mathbb{Z}_{l}\left(N^{\prime}\right)$ and $F_{2}\left(\varphi_{N}, l\right) \simeq F_{2}\left(\varphi_{N^{\prime}}, l\right)$. So

$$
\underline{\operatorname{GTM}}(N)_{l} \simeq \underline{\operatorname{GTM}}\left(N / l^{\alpha}\right)_{l} .
$$


This is consistent with the categorical interpretation of $\operatorname{GTM}(N)_{l}$, since the image of $K_{n, N}$ is an $l$-group iff the image of $K_{n, N^{\prime}}$ is, as $K_{n, N}$ is a normal subgroup of $K_{n, N^{\prime}}$ and the quotient in an $l$-group, so $\mathrm{Cat}_{N}=\mathrm{Cat}_{N / l^{\alpha}}$.

6.4. The morphism $\underline{\widehat{G T M}} \rightarrow \underline{\operatorname{GTM}}(N)_{l}$. Recall that we have a group morphism $\widehat{F}_{2} \rightarrow$ $\left.\widehat{F}_{2} \rightarrow \quad \overline{F_{2}\left(\varphi_{N}\right.}, l\right)$

$F_{2}\left(\varphi_{N}, l\right)$ such that $\searrow \quad \swarrow$ commutes. In the same way, we have an additive $\mathbb{Z} / N \mathbb{Z}$

group morphism $\widehat{\mathbb{Z}} \rightarrow \mathbb{Z}_{l}(N)$; this is actually a ring morphism, since we have a ring isomorphism $\widehat{\mathbb{Z}} \simeq \widehat{\mathbb{Z}}(N)$, which we compose with the morphism $\widehat{\mathbb{Z}}(N) \rightarrow \mathbb{Z}_{l}(N)$ induced by the projection $\widehat{\mathbb{Z}} \rightarrow \mathbb{Z}_{l}$. These induce maps $(1+2 \widehat{\mathbb{Z}}) \times \widehat{\mathbb{Z}} \times \widehat{F}_{2} \times \widehat{F}_{2} \rightarrow\left(1+2 \mathbb{Z}_{l}\right) \times \mathbb{Z}_{l}(N) \times\left(F_{2}\right)_{l} \times F_{2}\left(\varphi_{N}, l\right)$; one checks that this induces a semigroup morphism $\underline{\widehat{\mathrm{GT}}} \ltimes \widehat{\mathbb{Z}} \rightarrow \underline{\widehat{\mathrm{GTM}}} \rightarrow \underline{\operatorname{GTM}}(N)_{l}$, such that

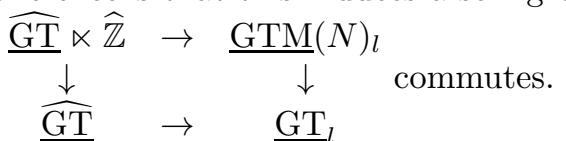

We have a semigroup morphism $\underline{\operatorname{GTM}}(N)_{l} \rightarrow \mathbb{Z}_{l}(N)=(\mathbb{Z} / N \mathbb{Z}) \times \mathbb{Z}_{l}$ (with multiplicative structure), $\left(\lambda, \lambda^{\prime}, f, g\right) \mapsto \lambda^{\prime}$. The composed map $\widehat{\mathbb{Z}} \subset \widehat{\mathrm{GT}} \ltimes \widehat{\mathbb{Z}} \rightarrow \widehat{\mathbb{Z}}(N)$ is trivial. Let $\operatorname{GTM}(N)_{l}:=\underline{\operatorname{GTM}}(N)_{l}^{i n v}$. The composed map $\operatorname{Gal}(\overline{\mathbb{Q}} / \mathbb{Q}) \rightarrow \widehat{\widehat{G T}} \rightarrow \operatorname{GTM}(N)_{l} \rightarrow(\mathbb{Z} / N \mathbb{Z})^{\times} \times$ $\mathbb{Z}_{l}^{\times}$is the cyclotomic character. This implies that the group morphism $\operatorname{Gal}(\overline{\mathbb{Q}} / \mathbb{Q}) \rightarrow \operatorname{GTM}(N)_{l}$ restricts to $\operatorname{Gal}\left(\overline{\mathbb{Q}} / \mathbb{Q}\left(\mu_{N l^{\infty}}\right)\right) \rightarrow \operatorname{GTM}_{(\overline{1}, 1)}(N)_{l}$, where $\operatorname{GTM}_{(\overline{1}, 1)}(N)_{l}$ is the preimage of the unit element $(\overline{1}, 1)$ by $\operatorname{GTM}(N)_{l} \rightarrow(\mathbb{Z} / N \mathbb{Z})^{\times} \times \mathbb{Z}_{l}^{\times}$.

6.5. The semigroup $\underline{\operatorname{GTM}}(N, \mathbf{k})$. Recall that $\underline{\operatorname{GT}}(\mathbf{k})$ is the set of pairs $(\lambda, f) \in \mathbf{k} \times F_{2}(\mathbf{k})$, satisfying the field versions of the defining conditions on GT; the hexagon and duality conditions take place in $F_{2}(\mathbf{k})$ and the pentagon condition in $P_{4}(\mathbf{k})$. This is a semigroup, which acts on braided monoidal categories $\mathcal{C}$ over $\mathbf{k}[[\hbar]]$, such that the images of the pure braid groups are of the form id $+O(\hbar)$; and also on QTQBA's over $\mathbf{k}[[\hbar]]$, such that $R=1^{\otimes 2}+O(\hbar)$ and $\Phi_{A}=$ $1^{\otimes 3}+O(\hbar)$. We have a semigroup morphism $\underline{\mathrm{GT}}(\mathbf{k}) \rightarrow \mathbf{k},(\lambda, f) \mapsto \lambda$, and $\mathrm{GT}(\mathbf{k}):=\underline{\mathrm{GT}}(\mathbf{k})^{\text {inv }}$ is the preimage of $\mathbf{k}^{\times}$.

We define $\underline{\operatorname{GTM}}(N, \mathbf{k})$ as the set of $(\lambda, \mu, f, g) \in \mathbf{k} \times \mathbf{k}(N) \times F_{2}(\mathbf{k}) \times F_{2}\left(\varphi_{N}, \mathbf{k}\right)$, satisfying the field octogon condition (in $F_{2}\left(\varphi_{N}, \mathbf{k}\right)$ ) and mixed pentagon condition (in $P_{4}\left(\varphi_{3, N}, \mathbf{k}\right)$ ). As before, if $(\lambda, \mu, f, g) \in \underline{\operatorname{GTM}}(N, \mathbf{k})$, then $\lambda=[\mu]$ and $g \in \operatorname{Ker} \varphi_{N}(\mathbf{k})=\left(\operatorname{Ker} \varphi_{N}\right)(\mathbf{k})$, where $\varphi_{N}(\mathbf{k}): F_{2}(\mathbf{k}) \rightarrow \mathbb{Z} / N \mathbb{Z}$ is induced by $\varphi_{N}$.

We have a semigroup morphism $\underline{\operatorname{GTM}}(N, \mathbf{k}) \rightarrow \mathbf{k}(N) \simeq(\mathbb{Z} / N \mathbb{Z}) \times \mathbf{k},(\lambda, \mu, f, g) \mapsto \mu$. One checks that the group $\operatorname{GTM}(N, \mathbf{k}):=\underline{\operatorname{GTM}}(N, \mathbf{k})^{i n v}$ coincides with the preimage of $\mathbf{k}(N)^{\times}=$ $(\mathbb{Z} / N \mathbb{Z})^{\times} \times \mathbf{k}^{\times}$.

We also have a semigroup morphism $\underline{\operatorname{GTM}}(N, \mathbf{k}) \rightarrow \underline{\mathrm{GT}}(\mathbf{k})$. The semigroup $\underline{\operatorname{GTM}}(N, \mathbf{k})$ acts on pairs $(\mathcal{C}, \mathcal{M})$ defined over $\mathbf{k}[[\hbar]]$, such that the images of $K_{n, N}$ are of the form id $+O(\hbar)$, and also on pairs $(A, B)$, where $A$ is a QTQBA as above and $B$ is a QRA over it, such that $E^{N}=1 \otimes 1+O(\hbar)$.

Let us prove that the map $F_{2}\left(\varphi_{N}, l\right) \rightarrow F_{2}\left(\varphi_{N}, \mathbb{Q}_{l}\right)$ is injective. For this, it suffices to prove that $\left(\operatorname{Ker} \varphi_{N}\right)_{l} \rightarrow\left(\operatorname{Ker} \varphi_{N}\right)\left(\mathbb{Q}_{l}\right)$ is injective. This follows from the fact that $\operatorname{Ker} \varphi_{N}$ is the free group with generators $X, y(\alpha), \alpha=0, \ldots, N-1$ (where $X:=x^{N}$ and $y(\alpha):=x^{\alpha} y x^{-\alpha}$ ).

The inclusion $\left(2 \mathbb{Z}_{l}+1\right) \times \mathbb{Z}_{l}(N) \times\left(F_{2}\right)_{l} \times F_{2}\left(\varphi_{N}, l\right) \subset \mathbb{Q}_{l} \times \mathbb{Q}_{l}(N) \times F_{2}\left(\mathbb{Q}_{l}\right) \times F_{2}\left(\varphi_{N}, \mathbb{Q}_{l}\right)$ then gives rise to a semigroup inclusion $\underline{\operatorname{GTM}}(N, l) \subset \underline{\operatorname{GTM}}\left(N, \mathbb{Q}_{l}\right)$ and an inclusion between the corresponding groups. 
6.6. The Lie algebra $\mathfrak{g t m}(N, \mathbf{k})$. Define the Magnus semigroup $\operatorname{Mag}(N)_{l}:=\{(\lambda, \mu, f, g) \in$ $\left.\mathbb{Z}_{l} \times \mathbb{Z}_{l}(N) \times\left(F_{2}\right)_{l} \times\left(\operatorname{Ker} \varphi_{N}\right)_{l} \mid \lambda=[\mu]\right\}$, equipped with the composition law of $\operatorname{GTM}(N)_{l}$. We have a morphism $\underline{\operatorname{Mag}}(N)_{l} \rightarrow \operatorname{End}\left(\left(F_{2}\right)_{l}\right)^{o p} \oplus \operatorname{End}\left(F_{2}\left(\varphi_{N}, l\right)\right)^{o p},(\lambda, \mu, f, g) \mapsto\left(\theta_{(\lambda, f)}, \theta_{(\lambda, \mu, g)}\right)$.

In the same way, one define $\operatorname{Mag}(N, \mathbf{k}):=\left\{(\lambda, \mu, f, g) \in \mathbf{k} \times \mathbf{k}(N) \times F_{2}(\mathbf{k}) \times\left(\operatorname{Ker} \varphi_{N}\right)(\mathbf{k}) \mid \lambda=\right.$ $[\mu]\}$ equipped with the composition law of $\operatorname{GTM}(N, \mathbf{k})$. We also have semigroup morphisms $\operatorname{Mag}(N, \mathbf{k}) \rightarrow \operatorname{End}\left(F_{2}(\mathbf{k})\right)^{o p} \oplus \operatorname{End}\left(F_{2}\left(\varphi_{N}, \mathbf{k}\right)\right)$ and $\operatorname{Mag}(N, \mathbf{k}) \rightarrow \mathbf{k}(N),(\lambda, \ldots) \mapsto \mu$. Let $\overline{\operatorname{Mag}}(N, \mathbf{k}):=\underline{\operatorname{Mag}}(N, \mathbf{k})^{i n v}$ be the preimage of $\mathbf{k}(N)^{\times} \cdot \overline{\operatorname{Let}} \operatorname{Mag}_{(\overline{1}, 1)}(N, \mathbf{k}),\left(\operatorname{resp} ., \operatorname{Mag}_{(\overline{1}, 1)}(N, \mathbf{k})\right)$ be the preimage of $(\overline{1}, 1)$ under $\underline{\operatorname{Mag}}(N, \mathbf{k}) \rightarrow \mathbf{k}(N)^{\times}$, resp., of $\overline{1}$ under $\underline{\operatorname{Mag}}(N, \mathbf{k}) \rightarrow(\mathbb{Z} / N \mathbb{Z})^{\times}$. We have an exact sequence $1 \rightarrow \overline{\operatorname{Mag}}_{(\overline{1}, 1)}(N, \mathbf{k}) \rightarrow \operatorname{Mag}_{\overline{1}}(N, \mathbf{k}) \rightarrow \mathbf{k}^{\times} \rightarrow \overline{1}$.

Let $\hat{\mathfrak{f}}\left(u_{i}, \in I\right)$ be the topologically free Lie algebra generated by $u_{i}, i \in I$. As $\operatorname{Ker} \varphi_{N}$ is the free group generated by $X:=x^{N}$ and $y(\alpha):=x^{\alpha} y x^{-\alpha}, \alpha \in[0, N-1], \operatorname{Lie}\left(\operatorname{Ker} \varphi_{N}\right)(\mathbf{k})=$ $\hat{\mathfrak{f}}(\Xi, \eta(\alpha), \alpha \in[0, N-1])$; here $\Xi:=\log X$ and $\eta(\alpha):=\log y(\alpha)$; similarly Lie $F_{2}(\mathbf{k})=\hat{\mathfrak{f}}_{2}(\xi, \eta)$, where $\xi:=\log x$ and $\eta:=\log y$.

Lemma 6.5. $\operatorname{Mag}_{(1, \overline{1})}(N, \mathbf{k})$ is a prounipotent Lie group. The Lie algebra of $\operatorname{Mag}_{\overline{1}}(\mathbf{k})$ is $\mathbf{k} \times$ Lie $F_{2}(\mathbf{k}) \times \operatorname{Lie}\left(\operatorname{Ker} \varphi_{N}\right)(\mathbf{k}) \simeq \mathbf{k} \times \hat{\mathfrak{f}}(\xi, \eta) \times \hat{\mathfrak{f}}(\Xi, \eta(\alpha), \alpha \in[0, N-1])$, equipped with the Lie bracket

$$
\left[\left(s_{1}, \varphi_{1}, \psi_{1}\right),\left(s_{2}, \varphi_{2}, \psi_{2}\right)\right]=\left(0,\left\langle\varphi_{1}, \varphi_{2}\right\rangle,\left\langle\psi_{1}, \psi_{2}\right\rangle\right),
$$

where

$$
\left\langle\varphi_{1}, \varphi_{2}\right\rangle=\left[\varphi_{1}, \varphi_{2}\right]+s_{2} D\left(\varphi_{1}\right)-s_{1} D\left(\varphi_{2}\right)+D_{\varphi_{2}}\left(\varphi_{1}\right)-D_{\varphi_{1}}\left(\varphi_{2}\right) ;
$$

where $D, D_{\varphi}$ are the derivations of $\hat{\mathfrak{f}}(\xi, \eta)$ defined by $D(\xi)=\xi, D(\eta)=\eta$ and $D_{\varphi}(\xi)=[\varphi, \xi]$, $D_{\varphi}(\eta)=0$, and

$$
\left\langle\psi_{1}, \psi_{2}\right\rangle=\left[\psi_{1}, \psi_{2}\right]+s_{2} \bar{D}\left(\psi_{1}\right)-s_{1} \bar{D}\left(\psi_{2}\right)+\bar{D}_{\psi_{2}}\left(\psi_{1}\right)-\bar{D}_{\psi_{1}}\left(\psi_{2}\right)
$$

here $\bar{D}, \bar{D}_{\psi}$ are the derivations of $\hat{\mathfrak{f}}(\Xi, \eta(0), \ldots, \eta(N-1))$ defined by

$$
\bar{D}: \Xi \mapsto \Xi, \eta(\alpha) \mapsto \eta(\alpha)+\frac{\alpha}{N}[\Xi, \eta(\alpha)](\alpha \in[0, N-1])
$$

$\bar{D}_{\psi}: \Xi \mapsto[\psi, \Xi], \eta(\alpha) \mapsto[\psi(\Xi \mid \eta(0), \ldots, \eta(N-1))-\psi(\Xi \mid \eta(\alpha), \ldots, \eta(\alpha+N-1)), \eta(\alpha)](\alpha \in[0, N-1])$ (one checks that these formulas hold for any $\alpha \in \mathbb{Z}$ ).

Proof. Identify $(\lambda, \mu, f, g)$ with $(a, k, f, g)$, where $\mu=(a, k) \in \mathbf{k}(N)$; so $\lambda=\tilde{a}+N k$.

The composition in $\operatorname{Mag}(N, \mathbf{k})$ is given by $\left(a_{1}, k_{1}, f_{1}, g_{1}\right)\left(a_{2}, k_{2}, f_{2}, g_{2}\right)=(a, k, f, g)$, where $a=a_{1} a_{2}, k$ is such that $\tilde{a}+N k=\left(\tilde{a}_{1}+N k_{1}\right)\left(\tilde{a}_{2}+N k_{2}\right), f(x, y)$ is given by $(21)$ and

$$
\begin{aligned}
& g(X \mid y(0), \ldots, y(N-1))=g_{2}(X \mid y(0), \ldots, y(N-1)) \cdot \\
& g_{1}\left(X^{\tilde{a}_{2}+N k_{2}} \mid \operatorname{Ad}\left(g_{2}(X \mid y(0), \ldots, y(N-1))^{-1}\right)\left(y(0)^{\tilde{a}_{2}+N k_{2}}\right),\right. \\
& \operatorname{Ad}\left(X^{k_{2}} g_{2}\left(X \mid y\left(\tilde{a}_{2}\right), \ldots, y\left(\tilde{a}_{2}+N-1\right)\right)^{-1}\right)\left(y\left(\tilde{a}_{2}\right)^{\tilde{a}_{2}+N k_{2}}\right), \ldots, \\
& \left.\operatorname{Ad}\left(X^{(N-1) k_{2}} g_{2}\left(X \mid y\left((N-1) \tilde{a}_{2}\right), \ldots, y\left((N-1) \tilde{a}_{2}+N-1\right)\right)^{-1}\right)\left(\left(y(N-1) \tilde{a}_{2}\right)^{\tilde{a}_{2}+N k_{2}}\right)\right) .
\end{aligned}
$$

Here we extend $\alpha \mapsto y(\alpha)$ to $\alpha \in \mathbb{Z}$ by $y(\alpha+N)=X y(\alpha) X^{-1}$. It is then clear that $\operatorname{Mag}_{(\overline{1}, 1)}(N, \mathbf{k})$ is a prounipotent Lie group. The Lie algebra structure is obtained by linearization.

We will denote by $\operatorname{Exp}: \hat{\mathfrak{f}}(\xi, \eta) \times \hat{\mathfrak{f}}(\Xi, \eta(0), \ldots, \eta(N-1)) \rightarrow F_{2}(\mathbf{k}) \times\left(\operatorname{Ker} \varphi_{N}\right)(\mathbf{k})$ the exponential map of $\operatorname{Mag}_{(\overline{1}, 1)}(N, \mathbf{k})$.

$\operatorname{GTM}_{(\overline{1}, 1)}(N, \mathbf{k})$ has been defined as $\operatorname{GTM}(N, \mathbf{k}) \cap \operatorname{Mag}_{(\overline{1}, 1)}(N, \mathbf{k})$; we similarly set $\operatorname{GTM}_{\overline{1}}(N, \mathbf{k}):=$ $\operatorname{GTM}(N, \mathbf{k}) \cap \operatorname{Mag}_{\overline{1}}(N, \mathbf{k})$. We then have an exact sequence $1 \rightarrow \operatorname{GTM}_{(\overline{1}, 1)}(N, \mathbf{k}) \rightarrow \operatorname{GTM}_{\overline{1}}(N, \mathbf{k}) \rightarrow$ $\mathbf{k}^{\times}$. We denote by

$$
0 \rightarrow \mathfrak{g t m}_{(\overline{1}, 1)}(N, \mathbf{k}) \rightarrow \mathfrak{g t m}_{\overline{1}}(N, \mathbf{k}) \rightarrow \mathbf{k}
$$


the corresponding exact sequence of Lie algebras.

Lemma 6.6. The Lie subalgebra $\mathfrak{g t m}_{\overline{1}}(N, \mathbf{k}) \subset \mathbf{k} \times \hat{\mathfrak{f}}(\xi, \eta) \times \hat{\mathfrak{f}}(\Xi, \eta(0), \ldots, \eta(N-1))$ is the set of all $(s, \varphi, \psi)$ such that

$$
\varphi(\eta, \xi)=-\varphi(\xi, \eta), \varphi(\xi, \eta)+\varphi(\eta, \zeta)+\varphi(\zeta, \xi)+\frac{s}{2}(\xi+\eta+\zeta)=0,
$$

where $e^{\xi} e^{\eta} e^{\zeta}=1$,

$$
\varphi\left(\xi^{12}, \xi^{23} * \xi^{24}\right)+\varphi\left(\xi^{13} * \xi^{23}, \xi^{34}\right)=\varphi\left(\xi^{23}, \xi^{34}\right)+\varphi\left(\xi^{12} * \xi^{13}, \xi^{24} * \xi^{34}\right)+\varphi\left(\xi^{12}, \xi^{23}\right)
$$

(relation in $\operatorname{Lie}\left(P_{4}(\mathbf{k})\right)$, where $\left.\xi^{i j}=\log x_{i j}\right)$,

$$
\begin{aligned}
& \psi(\Xi \mid \eta(0), \ldots, \eta(N-1))-\psi(\Xi \mid \eta(1), \ldots, \eta(N)) \\
& +\psi\left(\Theta \mid \eta^{\prime}(1), \ldots, \eta^{\prime}(2-N)\right)-\psi\left(\Theta \mid \eta^{\prime}(0), \ldots, \eta^{\prime}(1-N)\right)+\frac{s}{2}(\eta(0)+\eta(1))+\frac{s}{N}(\Xi+\Theta)=0,
\end{aligned}
$$

where $\eta(\alpha+N)=\operatorname{Ad}\left(e^{\Xi}\right)(\eta(\alpha)), e^{\Theta}=e^{-\eta(0)} \cdots e^{-\eta(1-N)} e^{-\Xi}, \eta^{\prime}(1)=\eta(1), \eta^{\prime}(0)=\eta(0)$, $\eta^{\prime}(-1)=\operatorname{Ad}\left(e^{-\eta(0)}\right)(\eta(-1)), \eta^{\prime}(-2)=\operatorname{Ad}\left(e^{-\eta(0)} e^{-\eta(-1)}\right)(\eta(-2))$, etc., and in general $\eta^{\prime}(\alpha-$ $N)=\operatorname{Ad}\left(e^{\Theta}\right)\left(\eta^{\prime}(\alpha)\right)$, and

$$
\begin{aligned}
& \psi\left(\Xi^{01} \mid \xi(0)^{12} * \xi(0)^{13}, \ldots, \xi(N-1)^{12} * \xi(N-1)^{13}\right) \\
& +\psi\left(\Xi^{02} * \xi(0)^{12} * \cdots * \xi(N-1)^{12} \mid \tilde{\xi}(0), \ldots, \tilde{\xi}(N-1)\right) \\
& =\varphi\left(\xi(0)^{12}, \xi(0)^{23}\right)+\psi\left(\Xi^{01} \mid \xi(0)^{12}, \ldots, \xi(N-1)^{12}\right) \\
& +\psi\left(\Xi^{01} * \Xi^{02} * \xi(0)^{12} * \cdots * \xi(N-1)^{12} *\left(-N \xi(0)^{12}\right) \mid \tilde{\tilde{\xi}}(0), \ldots, \tilde{\tilde{\xi}}(N-1)\right),
\end{aligned}
$$

(equality in $\operatorname{Lie}\left(K_{3, N}(\mathbf{k})\right)$ ) where $\tilde{\xi}(\alpha):=\log \operatorname{Ad}\left[x_{12}(1) \ldots x_{12}(\alpha) u_{12}(\alpha,-\alpha)\right]\left[x_{23}(\alpha)\right]$ and $\tilde{\tilde{\xi}}(\alpha):=$ $\log \operatorname{Ad}\left[x_{12}(0)^{-\alpha} x_{12}(1) \ldots x_{12}(\alpha)\right]\left[x_{13}(\alpha)\right] \operatorname{Ad}\left[x_{12}(0)^{-\alpha} x_{12}(-\alpha) \ldots x_{12}(-1)\right]\left[x_{23}(\alpha)\right]$; recall that $\log :$ $X_{0 i}, x_{i}(\alpha) \mapsto \Xi^{0 i}, \xi(\alpha)^{i j}$ and the $u_{i j}(\alpha, \beta)$ have been defined in Proposition 1.1.

The Lie algebra $\mathfrak{g t m}_{(\overline{1}, 1)}(N, \mathbf{k})$ is prounipotent and the $\operatorname{map} \mathfrak{g t m}_{\overline{1}}(N, \mathbf{k}) \rightarrow \mathbf{k}$ is $(s, \varphi, \psi) \mapsto s$.

Proof. The quadruple $(\lambda, \mu, f, g) \in \mathbf{k} \times \mathbf{k}(N) \times F_{2}(\mathbf{k}) \times\left(\operatorname{Ker} \varphi_{N}\right)(\mathbf{k})$ lies in $\underline{\operatorname{GTM}}(N, \mathbf{k})$ iff it satisfies $\lambda=[\mu]$, i.e., $\lambda=\tilde{a}+N k$ if $\mu=(a, k),(\lambda, f) \in \underline{\mathrm{GT}}(\mathbf{k})$,

$$
\begin{aligned}
& g(X \mid y(1), \ldots, y(N))^{-1} y(1)^{(\lambda-1) / 2} \\
& g\left(y(0)^{-1} \cdots y(1-N)^{-1} X^{-1} \mid\right. \\
& \left.y(1), y(0), \operatorname{Ad}\left(y(0)^{-1}\right)(y(-1)), \ldots, \operatorname{Ad}\left(y(0)^{-1} \cdots y(3-N)^{-1}\right)(y(2-N))\right) \\
& \left(y(0)^{-1} \cdots y(1-N)^{-1} X^{-1}\right)^{k} y(0)^{-1} y(-1)^{-1} \cdots y(2-\tilde{a})^{-1} \\
& g\left(y(1-\tilde{a})^{-1} \cdots y(2-\tilde{a}-N)^{-1} X^{-1} \mid\right. \\
& \left.y(1-\tilde{a}), \operatorname{Ad}\left(y(1-\tilde{a})^{-1}\right)(y(-\tilde{a})), \ldots, \operatorname{Ad}\left(y(1-\tilde{a})^{-1} \cdots y(3-\tilde{a}-N)^{-1}\right)(y(2-\tilde{a}-N))\right)^{-1} \\
& y(1-\tilde{a})^{(\lambda-1) / 2} g(X \mid y(1-\tilde{a}), \ldots, y(N-\tilde{a})) X^{k}=1,
\end{aligned}
$$

and equation (24), where $\partial_{0}: F_{2}(\mathbf{k}) \rightarrow K_{3, N}(\mathbf{k})$ is induced by $x \mapsto x_{12}(0), y \mapsto x_{23}(0)$ and $\partial_{i}:\left(\operatorname{Ker} \varphi_{N}\right)(\mathbf{k}) \rightarrow K_{3, N}(\mathbf{k})$ are given by

$$
\begin{gathered}
\partial_{1}: X \mapsto X_{02} x_{12}(0) \ldots x_{12}(N-1), \quad y(\alpha) \mapsto \operatorname{Ad}\left[x_{12}(1) \ldots x_{12}(\alpha) u_{12}(\alpha,-\alpha)\right]\left[x_{23}(\alpha)\right], \\
\partial_{2}: X \mapsto X_{01} X_{02} x_{12}(0) \ldots x_{12}(N-1) x_{12}(0)^{-N}, \\
y(\alpha) \mapsto \operatorname{Ad}\left[x_{12}(0)^{-\alpha} x_{12}(1) \cdots x_{12}(\alpha)\right]\left[x_{13}(\alpha)\right] \operatorname{Ad}\left[x_{12}(0)^{-\alpha} x_{12}(-\alpha) \cdots x_{12}(-1)\right]\left[x_{23}(\alpha)\right], \\
\partial_{3}: X \mapsto X_{01}, \quad y(\alpha) \mapsto x_{12}(\alpha) x_{13}(\alpha), \quad \partial_{4}: X \mapsto X_{01}, \quad y(\alpha) \mapsto x_{12}(\alpha) .
\end{gathered}
$$

The formulas are then obtained by setting $a=\overline{1}, \lambda=1+\varepsilon s, f(x, y)=e^{\varepsilon \varphi(\xi, \eta)}, g(X \mid y(0), \ldots, y(N-$ $1))=e^{\varepsilon \psi(\Xi \mid \eta(0), \ldots, \eta(N-1))}$ and linearizing in $\varepsilon$. 
Remark 6.7. As in $\left[\right.$ Dr2], the surjectivity of $\mathfrak{g t m}_{\overline{1}}(N, \mathbf{k}) \rightarrow \mathbf{k}$ for $\mathbf{k}=\mathbb{Q}_{l}$, hence $\mathbf{k}=\mathbb{Q}$, follows from that of the cyclotomic character $\operatorname{Gal}\left(\overline{\mathbb{Q}} / \mathbb{Q}\left(\mu_{N}\right)\right) \rightarrow \mathbb{Z}_{l}^{\times}$, and another proof of this surjectivity can be given using cyclotomic associators (see next section).

\section{Torsor structure of $\mathbf{P s e u d o}(N, \mathbf{k})$ and the $\operatorname{group} \operatorname{GrTM}(N, \mathbf{k})$}

7.1. Action of $\underline{\operatorname{GTM}}(N, \mathbf{k})$ on $\underline{\operatorname{Pseudo}}(N, \mathbf{k})$. The semigroup $\underline{\operatorname{GTM}}(N, \mathbf{k})$ acts on $\underline{\operatorname{Pseudo}}(N, \mathbf{k})$ as follows:

$$
(\lambda, \mu, f, g) *\left(a^{\prime}, \lambda^{\prime}, \Phi^{\prime}, \Psi^{\prime}\right)=\left(\bar{\mu} a^{\prime},[\mu] \lambda^{\prime}, \Phi^{\prime \prime}, \Psi^{\prime \prime}\right)
$$

where

$$
\begin{gathered}
\Phi^{\prime \prime}\left(t^{12}, t^{23}\right):=\Phi^{\prime}\left(t^{12}, t^{23}\right) f\left(e^{\lambda^{\prime} t^{12}}, \operatorname{Ad}\left(\Phi^{\prime}\left(t^{12}, t^{23}\right)^{-1}\right)\left(e^{\lambda^{\prime} t^{23}}\right)\right), \\
\Psi^{\prime \prime}\left(t_{0}^{12} \mid t(0)^{23}, \ldots, t(N-1)^{23}\right):=\Psi^{\prime}\left(t_{0}^{12} \mid t(0)^{23}, \ldots, t(N-1)^{23}\right) \\
g\left(e^{\lambda^{\prime} t_{0}^{12}} \mid \operatorname{Ad}\left(\Psi^{\prime}\left(t_{0}^{12} \mid t(0)^{23}, \ldots, t(N-1)^{23}\right)^{-1}\right)\left(e^{\lambda^{\prime} t(0)^{23}}\right),\right. \\
\operatorname{Ad}\left(e^{\left(\lambda^{\prime} / N\right) t_{0}^{12}} \Psi^{\prime}\left(t_{0}^{12} \mid t\left(a^{\prime}\right)^{23}, \ldots, t\left(a^{\prime}+N-1\right)^{23}\right)^{-1}\right)\left(e^{\lambda^{\prime} t\left(a^{\prime}\right)^{23}}\right), \ldots, \\
\left.\operatorname{Ad}\left(e^{(N-1)\left(\lambda^{\prime} / N\right) t_{0}^{12}} \Psi^{\prime}\left(t_{0}^{12} \mid t\left((N-1) a^{\prime}\right)^{23}, \ldots, t\left((N-1) a^{\prime}+N-1\right)^{23}\right)^{-1}\right)\left(e^{\lambda^{\prime} t\left((N-1) a^{\prime}\right)^{23}}\right)\right)
\end{gathered}
$$

(recall that $\lambda=[\mu]$, so if $\mu=(a, k)$, then $\lambda=\tilde{a}+N k$; also $\bar{\mu}=a)$. This action is compatible with the action of $\underline{\mathrm{GT}}(\mathbf{k})$ on $\underline{\operatorname{Assoc}}(\mathbf{k})$ and the natural morphisms $\underline{\operatorname{GTM}}(N, \mathbf{k}) \rightarrow \underline{\mathrm{GT}}(\mathbf{k})$, $\underline{\operatorname{Pseudo}}(N, \mathbf{k}) \rightarrow \underline{\operatorname{Assoc}}(\mathbf{k})$.

This action restricts to an action of $\operatorname{GTM}(N, \mathbf{k})$ on $\operatorname{Pseudo}(N, \mathbf{k})$, compatible with the action of $\mathrm{GT}(\mathbf{k})$ on $\operatorname{Assoc}(\mathbf{k})$.

Theorem 7.1. If $\mathbf{P s e u d o}(N, \mathbf{k})$ is nonempty, then the action of $\operatorname{GTM}(N, \mathbf{k})$ on $\mathbf{P s e u d o}(N, \mathbf{k})$ is free and transitive, in other words, $\operatorname{Pseudo}(N, \mathbf{k})$ is a torsor under $\operatorname{GTM}(N, \mathbf{k})$.

Proof. The proof is parallel to [Dr2], Proposition 5.1. One shows that formula (28) defines a free and transitive action of $\operatorname{Mag}(N, \mathbf{k})$ on $\left\{(a, \lambda, f, g) \in(\mathbb{Z} / N \mathbb{Z})^{\times} \times \mathbf{k}^{\times} \times F_{2}(\mathbf{k}) \times\left(\operatorname{Ker} \varphi_{N}\right)(\mathbf{k})\right\}$. Hence given $\left(a_{i}^{\prime}, \lambda_{i}^{\prime}, \Phi_{i}, \Psi_{i}\right), i=0,1$ in $\operatorname{Pseudo}(N, \mathbf{k})$, there is a unique $(\lambda, \mu, f, g) \in \operatorname{Mag}(N, \mathbf{k})$, such that $(\lambda, \mu, f, g) *\left(a_{0}^{\prime}, \lambda_{0}^{\prime}, \Phi_{0}, \Psi_{0}\right)=\left(a_{1}^{\prime}, \lambda_{1}^{\prime}, \Phi_{1}, \Psi_{1}\right)$. It remains to show that $(a, \lambda, f, g) \in$ $\operatorname{GTM}(N, \mathbf{k})$. [Dr2], Proposition 5.1 implies that $(\lambda, f)$ satisfies (16), (17). Let $\rho_{\left(a^{\prime}, \lambda^{\prime}, \Phi, \Psi\right)}^{n}$ : $B_{n}^{1}\left(\varphi_{n, N}, \mathbf{k}\right) \rightarrow \exp \left(\hat{\mathfrak{t}}_{n, N}(\mathbf{k})\right) \rtimes(\mathbb{Z} / N \mathbb{Z})^{n} \rtimes \mathfrak{S}_{n}$ be the morphism induced by $\left(a^{\prime}, \lambda^{\prime}, \Phi, \Psi\right) \in$ $\operatorname{Pseudo}(N, \mathbf{k})$. Replacing in the identities (8), (9) satisfied by $\left(\Phi_{1}, \Psi_{1}\right), \Phi_{1}$ and $\Psi_{1}$ by their expressions in terms of $(f, g)$ and $\left(\Phi_{0}, \Psi_{0}\right)$, and using the fact that $\left(\Phi_{0}, \Psi_{0}\right)$ satisfies the same identities, we find that the images of relations (27), (24) by $\rho_{\left(a_{0}^{\prime}, \lambda_{0}^{\prime}, \Phi_{0}, \Psi_{0}\right)}(n=2,3)$ are satisfied; since $\rho_{\left(a_{0}^{\prime}, \lambda_{0}^{\prime}, \Phi_{0}, \Psi_{0}\right)}^{n}$ is bijective, these relations are satisfied in $B_{n}^{1}\left(\varphi_{n, N}, \mathbf{k}\right)(n=2,3)$.

7.2. Pseudotwists and splittings of exact sequences. The group $(\mathbb{Z} / N \mathbb{Z})^{\times} \times \mathbf{k}^{\times}$acts by automorphisms of $\mathfrak{t}_{n, N}$ as follows: $(c, \gamma) \cdot t_{0}^{1 i}=\gamma t_{0}^{1 i},(c, \gamma) \cdot t(a)^{i j}=\gamma t(c a)^{i j}$, and it acts by automorphisms of $\mathfrak{t}_{n}$ by $(c, \gamma) \cdot t^{i j}=\gamma t^{i j}$. This induces an action of $(\mathbb{Z} / N \mathbb{Z})^{\times} \times \mathbf{k}^{\times}$on $\underline{\operatorname{Pseudo}}(N, \mathbf{k})$, such that the map $\underline{\operatorname{Pseudo}}(N, \mathbf{k}) \rightarrow \mathbb{Z} / N \mathbb{Z} \times \mathbf{k}$ is equivariant $(\mathbb{Z} / N \mathbb{Z} \times \mathbf{k}$ being equipped with the action $(c, \gamma) \cdot(a, \lambda)=(c a, \gamma \lambda))$. In particular, we get isomorphisms $\operatorname{Pseudo}_{(a, \lambda)}(N, \mathbf{k}) \simeq \operatorname{Pseudo}_{(\overline{1}, 1)}(N, \mathbf{k})$ for any $(a, \lambda) \in(\mathbb{Z} / N \mathbb{Z})^{\times} \times \mathbf{k}^{\times}$.

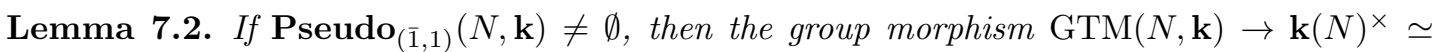
$(\mathbb{Z} / N \mathbb{Z})^{\times} \times \mathbf{k}^{\times}$is onto. 
Proof. Let $(a, \lambda) \in(\mathbb{Z} / N \mathbb{Z})^{\times} \times \mathbf{k}^{\times}$and $\Psi \in \operatorname{Pseudo}_{(\overline{1}, 1)}(N, \mathbf{k})$, then $(a, \lambda) \cdot \Psi \in \operatorname{Pseudo}_{(a, \lambda)}(N, \mathbf{k})$; according to Theorem 7.1, there is a unique $g \in \operatorname{GTM}(N, \mathbf{k})$ such that $g * \Psi=(a, \lambda) \cdot \Phi$. Then the image of $g$ under $\operatorname{GTM}(N, \mathbf{k}) \rightarrow \mathbf{k}(N)^{\times}$is $(a, \lambda)$.

It follows that if $\mathbf{P s e u d o}_{(\overline{1}, 1)}(N, \mathbf{k}) \neq \emptyset$, the morphisms $\operatorname{GTM}_{\overline{1}}(N, \mathbf{k}) \rightarrow \mathbf{k}^{\times}$and $\mathfrak{g t m}_{\overline{1}}(N, \mathbf{k}) \rightarrow$ $\mathbf{k}$ are also onto.

The actions of $(\mathbb{Z} / N \mathbb{Z})^{\times} \times \mathbf{k}^{\times}$and $\operatorname{GTM}(N, \mathbf{k})$ on $\operatorname{Pseudo}(N, \mathbf{k})$ commute with each other. We have therefore an action of $\operatorname{GTM}(N, \mathbf{k})$ on $\operatorname{Pseudo}_{(\overline{1}, 1)}(N, \mathbf{k})$, defined by $(\lambda, \mu, f, \bar{g}) \circ \Psi=$ $(\bar{\mu},[\mu])^{-1} \cdot(\lambda, \mu, f, \bar{g}) \Psi$.

To each $\Psi \in \mathbf{P s e u d o}_{(\overline{1}, 1)}(N, \mathbf{k})$, we associate the Lie algebra of the stabilizer of $\Psi$. This defines a section of the exact sequence

$$
0 \rightarrow \mathfrak{g t m}_{(\overline{1}, 1)}(N, \mathbf{k}) \rightarrow \mathfrak{g t m}_{\overline{1}}(N, \mathbf{k}) \rightarrow \mathbf{k} \rightarrow 0 .
$$

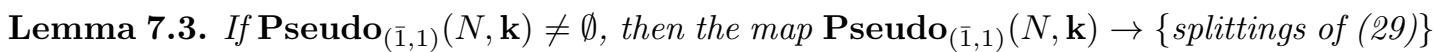
is a bijection.

Proof. $\operatorname{GTM}_{(\overline{1}, 1)}(N, \mathbf{k})$ acts on the target of this map by conjugation, and the map is then equivariant. So it suffices to show that $\operatorname{Spl}(\mathbf{k}):=\{$ splittings of $(29)\}$ is a torsor under $\operatorname{GTM}_{(\overline{1}, 1)}(N, \mathbf{k})$. $\operatorname{Spl}(\mathbf{k})$ identifies with $\left\{\right.$ elements of $\mathfrak{g t m}_{\overline{1}}(N, \mathbf{k})$ of the form $\left.(1, \varphi, \psi)\right\}$. If we fix an origin $\left(1, \varphi_{0}, \psi_{0}\right)$, then $\operatorname{Spl}(\mathbf{k})$ identifies with $\mathfrak{g t m}_{(\overline{1}, 1)}(N, \mathbf{k})$, and the vector field at $\left(0, \varphi^{\prime}, \psi^{\prime}\right) \in \mathfrak{g t m}_{(\overline{1}, 1)}(N, \mathbf{k}) \simeq \operatorname{Spl}(N, \mathbf{k})$ induced by the action of $(0, \varphi, \psi) \in \mathfrak{g t m}_{(\overline{1}, 1)}(N, \mathbf{k})$ is $\left(0,\left[\varphi_{0}, \varphi^{\prime}\right]+D\left(\varphi_{0}\right)+D_{\varphi_{0}}\left(\varphi^{\prime}\right)-D_{\varphi^{\prime}}\left(\varphi_{0}\right),\left[\psi_{0}, \psi^{\prime}\right]+\bar{D}\left(\psi_{0}\right)+\bar{D}_{\psi_{0}}\left(\psi^{\prime}\right)-\bar{D}_{\psi^{\prime}}\left(\psi_{0}\right)\right)$; this defines a linear map $\vartheta_{\left(0, \varphi^{\prime}, \psi^{\prime}\right)}: \mathfrak{g t m}_{(\overline{1}, 1)}(N, \mathbf{k}) \rightarrow \mathfrak{g t m}_{(\overline{1}, 1)}(N, \mathbf{k})$. Now $\mathfrak{g t m}_{(\overline{1}, 1)}(N, \mathbf{k})$ has a decreasing filtration $\mathfrak{g}^{\geq k}=\left\{(0, \varphi, \psi) \in \mathfrak{g t m}_{(\overline{1}, 1)}(N, \mathbf{k}) \mid \psi\right.$ has degree $\left.\geq k\right\}$ (this condition implies that $\varphi$ has also degree $\geq k), \vartheta_{\left(0, \varphi^{\prime}, \psi^{\prime}\right)}$ is compatible with this filtration and the associated graded map is multiplication by $k$ in degree $k$, so that $\vartheta_{\left(0, \varphi^{\prime}, \psi^{\prime}\right)}$ is bijective. This suffices to show that the action of $\operatorname{GTM}_{(\overline{1}, 1)}(N, \mathbf{k})$ is free, and using successive approximations, one can also show that it is transitive.

Proposition 7.4. If $\mathfrak{g t m}_{\overline{1}}(N, \mathbf{k}) \rightarrow \mathbf{k}$ is surjective, then $\operatorname{Pseudo}_{(\overline{1}, 1)}(N, \mathbf{k}) \neq \emptyset$.

The proof follows [Dr2], Proposition 5.2.

Proof. Let us assume that this map is surjective, i.e., there exists an element $(1, \varphi, \psi)$ in $\mathfrak{g t m}_{\overline{1}}(N, \mathbf{k})$. There exists a unique pair $(\Phi, \Psi)$, where $\Phi \in F_{2}(\mathbf{k})$ and $\Psi \in\left(\operatorname{Ker} \varphi_{N}\right)(\mathbf{k})$, such that

$$
\begin{gathered}
\Phi(U, V)^{-1} \frac{\mathrm{d}}{\mathrm{d} t}_{\mid t=1} \Phi(t U, t V)=\varphi\left(N U, \operatorname{Ad}\left(\Phi(U, V)^{-1}\right)(N V)\right), \\
\Psi(A \mid b(0), \ldots, b(N-1))^{-1} \frac{\mathrm{d}}{\mathrm{d} t}_{\mid t=1} \Psi(t A \mid t b(0), \ldots, t b(N-1)) \\
=\psi\left(N A \mid \operatorname{Ad}(\Psi(A \mid b(0), \ldots, b(N-1)))^{-1}(N b(0)), \operatorname{Ad}\left(e^{A} \Psi(A \mid b(1), \ldots, b(N))\right)^{-1}(N b(1)),\right. \\
\left.\ldots, \operatorname{Ad}\left(e^{(N-1) A} \Psi(A \mid b(N-1), \ldots, b(2 N-2))\right)^{-1}(N b(N-1))\right) .
\end{gathered}
$$

Proposition 5.2 in [Dr2] implies that $\Phi \in \operatorname{Assoc}_{N}(\mathbf{k})$. Repeating the final argument of loc. cit., we find that $(\Phi, \Psi)$ satisfies the mixed pentagon relation (9). Let us now show that $\Psi$ satisfies the octogon relation $(8)$, where $(\alpha, \lambda)=(\overline{1}, N)$. Let us set

$$
\begin{aligned}
& Q(A \mid b(0), \ldots, b(N-1)):=\Psi(C \mid b(0), \ldots, b(1-N)) e^{C} \Psi(C \mid b(-1), \ldots, b(-N))^{-1} e^{N b(-1) / 2} \\
& \Psi(A \mid b(-1), \ldots, b(N-2)) e^{A} \Psi(A \mid b(0), \ldots, b(N-1))^{-1} e^{N b(0) / 2} .
\end{aligned}
$$


We have $\Psi(A \mid b(0), \ldots, b(N-1))=1+\psi(A \mid b(0), \ldots, b(N-1))$ modulo degree $\geq 2$. Since $\psi$ satisfies $(26)$, we get $Q=1$ modulo degree $\geq 2$. Assume that we have proved that $Q=1$ modulo degree $\geq n$, where $n \geq 2$.

We have

$$
\begin{aligned}
& Q(A \mid b(0), \ldots, b(N-1))^{-1} \frac{\mathrm{d}}{\mathrm{d} t} \mid t=1 Q(t A \mid t b(0), \ldots, t b(N-1)) \\
& =\frac{N b(0)}{2}+A^{\prime}-\psi\left(N A^{\prime} \mid N b^{\prime}(0), \ldots, N b^{\prime}(N-1)\right)+\psi\left(N A^{\prime} \mid N b^{\prime}(-1), \ldots, N b^{\prime}(N-2)\right) \\
& +\frac{N b^{\prime}(-1)}{2}+C^{\prime}+\psi\left(N C^{\prime} \mid N b^{\prime \prime}(-1), \ldots, N b^{\prime \prime}(-N)\right)-\psi\left(N C^{\prime} \mid N b^{\prime \prime}(0), \ldots, N b^{\prime \prime}(1-N)\right),
\end{aligned}
$$

where

$$
\begin{gathered}
A^{\prime}=\operatorname{Ad}\left(e^{-N b(0) / 2} \Psi(A \mid b(0), \ldots, b(N-1))\right)(A), \\
b^{\prime}(a)=\operatorname{Ad}\left(e^{-N b(0) / 2} \Psi(A \mid b(0), \ldots, b(N-1)) e^{a A} \Psi(A \mid b(a), \ldots, b(a+N-1))^{-1}\right)(b(a)), \\
C^{\prime}=\operatorname{Ad}\left(e^{-N b(0) / 2} \Psi(A \mid b(0), \ldots, b(N-1)) e^{-A}\right. \\
\left.\Psi(A \mid b(-1), \ldots, b(N-2))^{-1} e^{-N b(-1) / 2} \Psi(C \mid b(-1), \ldots, b(-N))\right)(C), \\
b^{\prime \prime}(a)=\operatorname{Ad}\left(e^{-N b(0) / 2} \Psi(A \mid b(0), \ldots, b(N-1)) e^{-A} \Psi(A \mid b(-1), \ldots, b(N-2))^{-1}\right. \\
\left.e^{-N b(-1) / 2} \Psi(C \mid b(-1), \ldots, b(-N)) e^{-(a+1) C} \Psi(C \mid b(a), \ldots, b(a-N+1))^{-1}\right)(b(a)) .
\end{gathered}
$$

Define $q(A \mid b(0), \ldots, b(N-1))$ as $(Q-1$ modulo degree $\geq n+1)$. So the l.h.s. of (30) is $1+n q(A \mid b(0), \ldots, b(N-1))+($ degree $\geq n+1)$.

Let us compute the r.h.s. of (30). We have

$$
e^{N C^{\prime}} e^{N b^{\prime}(0)} \cdots e^{N b^{\prime}(N-1)} e^{N A^{\prime}}=1+\sum_{a=0}^{N-1} q(A \mid b(a), \ldots, b(a+N-1))+(\text { degree } \geq n+1),
$$

and

$$
\begin{aligned}
e^{N b^{\prime \prime}(-a-1)} & =\operatorname{Ad}\left(Q(A \mid b(0), \ldots, b(N-1))^{-1}\right)\left(e^{-N b^{\prime}(-1)} \cdots e^{-N b^{\prime}(-a)} e^{N b^{\prime}(-a-1)} \cdots e^{N b^{\prime}(-1)}\right) \\
& + \text { degree } \geq n+1 .
\end{aligned}
$$

Define $\bar{b}(-a)$ by

$$
\begin{aligned}
e^{N \bar{b}(-a-1)} & =e^{-N b^{\prime}(-1)} \cdots e^{-N b^{\prime}(-a)} e^{N b^{\prime}(-a-1)} \cdots e^{N b^{\prime}(-1)}, \\
C^{\prime \prime} & :=\operatorname{Ad}(\Psi(C \mid b(0), \ldots, b(1-N)))(C), \\
A^{\prime \prime} & :=A^{\prime}-\frac{1}{N} \sum_{a=0}^{N-1} q(A \mid b(a), \ldots, b(a+N-1)) .
\end{aligned}
$$

We have $C^{\prime}=\operatorname{Ad}\left(Q(A \mid b(0), \ldots, b(a+N-1))^{-1}\right)\left(C^{\prime \prime}\right)$, therefore $C^{\prime}=C^{\prime \prime}+$ degree $\geq n+1$. Therefore $e^{N C^{\prime \prime}} e^{N b^{\prime}(0)} \cdots e^{N b^{\prime}(N-1)} e^{N A^{\prime \prime}}=1+($ degree $\geq n+1)$. So $(26)$ implies

$$
\begin{aligned}
& \frac{N b(0)}{2}+A^{\prime \prime}-\psi\left(N A^{\prime \prime} \mid N b^{\prime}(0), \ldots, N b^{\prime}(N-1)\right)+\psi\left(N A^{\prime \prime} \mid N b^{\prime}(-1), \ldots, N b^{\prime}(N-2)\right) \\
& +\frac{N b^{\prime}(-1)}{2}+C^{\prime \prime}+\psi\left(N C^{\prime \prime} \mid N \bar{b}(-1), \ldots, N \bar{b}(-N)\right)-\psi\left(N C^{\prime \prime} \mid B \bar{b}(0), \ldots, \bar{b}(1-N)\right)=0 .
\end{aligned}
$$

So the r.h.s. of (30) is equal to

$$
A^{\prime}-A^{\prime \prime}+(\text { degree } \geq n+1)=\frac{1}{N} \sum_{a=0}^{N-1} q(A \mid b(a), \ldots, b(a+N-1))+(\text { degree } \geq n+1) .
$$


Therefore (30) implies

$$
n q(A \mid b(0), \ldots, b(N-1))=\frac{1}{N} \sum_{a=0}^{N-1} q(A \mid b(a), \ldots, b(a+N-1)) .
$$

This equation implies that $q(A \mid b(0), \ldots, b(N-1))$ is invariant under the automorphism $A \mapsto A$, $b(a) \mapsto b(a+1)$. So its r.h.s. is equal to $q(A \mid b(0), \ldots, b(N-1))$. Therefore the equation is rewritten as $(n-1) q(A \mid b(0), \ldots, b(N-1))=0$, which implies (since $n \geq 2) q(A \mid b(0), \ldots, b(N-$ $1))=0$, so $Q=1$ modulo degree $\geq n+1$. So $Q=1$. So $(\Phi, \Psi) \in \operatorname{Pseudo}_{(\overline{1}, N)}(N, \mathbf{k})$; hence Pseudo $_{(\overline{1}, 1)}(N, \mathbf{k}) \neq \emptyset$.

Corollary 7.5. $\operatorname{Pseudo}_{(\overline{1}, 1)}(N, \mathbb{Q}) \neq \emptyset$.

Proof. As in [Dr2], Pseudo $(\overline{1}, 1)(N, \mathbb{C}) \neq \emptyset$ implies that $\mathfrak{g t m}_{\overline{1}}(N, \mathbf{k}) \rightarrow \mathbf{k}$ is surjective for $\mathbf{k}=\mathbb{C}$. This implies the same statement for $\mathbf{k}=\mathbb{Q}$. Then Proposition 7.4 implies that $\operatorname{Pseudo}_{(\overline{1}, 1)}(N, \mathbb{Q}) \neq \emptyset$.

7.3. $\operatorname{GRTM}(N, \mathbf{k})$ and $\mathfrak{g r t m}(N, \mathbf{k}) \cdot$ Define $\operatorname{GRTM}_{(\overline{1}, 1)}(N, \mathbf{k})$ as the set of pairs $(h, k)$ with $h \in \exp \left(\hat{\mathfrak{t}}_{3}^{0}\right)$ and $k \in \exp \left(\hat{\mathfrak{t}}_{3, N}^{0}\right)$, such that (we recall that $U=t^{12}, V=t^{23}$, and $A=t_{0}^{01}$, $b(a)=t(a)^{12}$ for $\left.a \in \mathbb{Z} / N \mathbb{Z}\right)$

$$
\begin{gathered}
h(U, V)=h(V, U)^{-1}, \quad h(U, V) h(V, W) h(W, U)=1, \\
U+\operatorname{Ad}\left(h(U, V)^{-1}\right)(V)+\operatorname{Ad}\left(h(U, W)^{-1}\right)(W)=0 \text { for } U+V+W=0, \\
h^{1,2,34} h^{12,3,4}=h^{2,3,4} h^{1,23,4} h^{1,2,3}
\end{gathered}
$$

(equality in $\left.\exp \left(\hat{\mathfrak{t}}_{4}\right)\right)$, and

$$
\begin{aligned}
& k(A \mid b(1), \ldots, b(N))^{-1} k(C \mid b(1), b(0), \ldots, b(2-N)) \\
& k(C \mid b(0), b(-1), \ldots, b(1-N))^{-1} k(A \mid b(0), \ldots, b(N-1))=1, \\
A+ & \sum_{a=1}^{N} \operatorname{Ad}\left(k(A \mid b(a), \ldots, b(a+N-1))^{-1}\right)(b(a)) \\
+ & \operatorname{Ad}\left(k(A \mid b(0), \ldots, b(N-1))^{-1} k(C \mid b(0), \ldots, b(1-N))\right)(C)=0,
\end{aligned}
$$

where $A+\sum_{i=0}^{n} b(i)+C=0$,

$$
k^{0,1,23} k^{01,2,3}=h^{1,2,3} k^{0,12,3} k^{0,1,2}
$$

(equality in $\exp \left(\widehat{\mathfrak{t}}_{3, N}\right)$ ).

$\operatorname{GRTM}_{(\overline{1}, 1)}(N, \mathbf{k})$ is a group when equipped with the product $\left(h_{1}, k_{1}\right) *\left(h_{2}, k_{2}\right)=(h, k)$, where

$$
h(U, V)=h_{2}(U, V) h_{1}\left(U, \operatorname{Ad} h_{2}(U, V)^{-1}(V)\right),
$$

$k(A \mid b(0), \ldots, b(N-1))=k_{2}(A \mid b(0), \ldots, b(N-1)) \cdot$

$k_{1}\left(A \mid \operatorname{Ad}\left(k_{2}(A \mid b(0), \ldots, b(N-1))^{-1}\right)(b(0)), \ldots, \operatorname{Ad}\left(k_{2}(A \mid b(N-1), \ldots, b(2 N-2))^{-1}\right)(b(N-1))\right)$;

so we have a group inclusion $\operatorname{GRTM}_{(\overline{1}, 1)}(N, \mathbf{k}) \subset \operatorname{Mag}_{(\overline{1}, 1)}(N, \mathbf{k})$. The action of $(\mathbb{Z} / N \mathbb{Z})^{\times} \times \mathbf{k}^{\times}$ by automorphisms of $\mathfrak{t}_{3, N}^{0}$ and $\mathfrak{t}_{3}^{0}$ induces its action by automorphisms of $\operatorname{GRTM}_{(\overline{1}, 1)}(N, \mathbf{k})$. We denote by $\operatorname{GRTM}(N, \mathbf{k})$ the corresponding semidirect product.

The Lie algebra $\mathfrak{g r t m}_{(\overline{1}, 1)}(N, \mathbf{k})$ of $\operatorname{GRTM}_{(\overline{1}, 1)}(N, \mathbf{k})$ consists of all pairs $(\varphi, \psi) \in \hat{\mathfrak{t}}_{3}^{0} \times \hat{\mathfrak{t}}_{3, N}^{0}$, such that:

$$
\varphi(V, U)=-\varphi(U, V), \varphi(U, V)+\varphi(V, W)+\varphi(W, U)=0
$$


if $U+V+W=0$,

$$
\begin{gathered}
{[V, \varphi(U, V)]+[W, \varphi(U, W)]=0 \text { if } U+V+W=0,} \\
\varphi^{12,3,4}+\varphi^{1,2,34}=\varphi^{1,2,3}+\varphi^{1,23,4}+\varphi^{2,3,4}, \\
\psi(A \mid b(0), b(1), \ldots, b(N-1))-\psi(A \mid b(1), b(2), \ldots, b(0)) \\
+\psi(C \mid b(1), b(0), \ldots, b(2-N))-\psi(C \mid b(0), b(-1), \ldots, b(1-N))=0,
\end{gathered}
$$

where $C=-A-\sum_{i=0}^{N-1} b(i)$,

$[\psi(A \mid b(0), \ldots, b(N-1))-\psi(C \mid b(0), \ldots, b(1-N)), C]+\sum_{a=0}^{N-1}[\psi(A \mid b(a), \ldots, b(a+N-1)), b(a)]=0$

where $C=-A-\sum_{i=0}^{N-1} b(i)$

$$
\psi^{1,2,34}+\psi^{12,3,4}=\varphi^{1,2,3}+\psi^{1,23,4}+\psi^{1,2,3} .
$$

The Lie bracket is given by

$$
\left[\left(\varphi_{1}, \psi_{1}\right),\left(\varphi_{2}, \psi_{2}\right)\right]=\left(\left\langle\varphi_{1}, \varphi_{2}\right\rangle,\left\langle\psi_{1}, \psi_{2}\right\rangle\right)
$$

where

$$
\begin{aligned}
\left\langle\varphi_{1}, \varphi_{2}\right\rangle & =\left[\varphi_{1}, \varphi_{2}\right]+D_{\varphi_{2}}\left(\varphi_{1}\right)-D_{\varphi_{1}}\left(\varphi_{2}\right) \\
\left\langle\psi_{1}, \psi_{2}\right\rangle & =\left[\psi_{1}, \psi_{2}\right]+\bar{D}_{\psi_{2}}\left(\psi_{1}\right)-\bar{D}_{\psi_{1}}\left(\psi_{2}\right)
\end{aligned}
$$

Here $D_{\varphi}, \bar{D}_{\psi}$ are defined by

$$
D_{\varphi}(U)=[\varphi, U], D_{\varphi}(V)=0
$$

and

$$
\bar{D}_{\psi}(A)=[\psi, A], \bar{D}_{\psi}(b(a))=[\psi(A \mid b(0), \ldots, b(N-1))-\psi(A \mid b(a), \ldots, b(a+N-1)), b(a)] .
$$

The direct sum of its homogeneous components is a graded Lie subalgebra of $\mathfrak{g r t m}_{(\overline{1}, 1)}(N, \mathbf{k})$, and $\mathfrak{g r t m}_{(\overline{1}, 1)}(N, \mathbf{k})$ is then the corresponding graded completion.

The Lie algebra $\operatorname{grtm}(N, \mathbf{k})$ is the semidirect product $\mathfrak{g r t m}_{1}(N, \mathbf{k}) \rtimes \mathbf{k}, 1 \in \mathbf{k}$ acting by the continuous derivation taking an element $(\varphi, \psi)$ of degree $n$ to $-n(\varphi, \psi)$. Then $\mathfrak{g r t m}(N, \mathbf{k})$ is equipped with an action of $(\mathbb{Z} / N \mathbb{Z})^{\times}$.

Remark 7.6. The octogon condition (38) means that

$$
\psi(A \mid b(a), \ldots, b(a+N-1))-\psi(C \mid b(a), \ldots, b(a+1-N))
$$

is independent of $a$. In the same way, the octogon condition (33) means that $k(A \mid b(a), \ldots, b(a+$ $N-1))^{-1} k(C \mid b(a), b(a-1), \ldots, b(a+1-N))$ is independent of $a$. These facts are used when proving that $\operatorname{GRTM}_{(\overline{1}, 1)}(N, \mathbf{k})$ is a group.

Remark 7.7. (39) implies (38). Indeed, (39) implies that

$$
[\psi(A \mid b(0), \ldots, b(N-1))-\psi(C \mid b(0), \ldots, b(1-N)), C]
$$

is invariant under the automorphism $\theta_{1}: A \mapsto A, b(a) \mapsto b(a+1)$. So for some $\lambda \in \mathbf{k}$, we have l.h.s. of $(38)=\lambda C$. Applying id $+\theta_{1}+\cdots+\theta_{1}^{N-1}$ to this identity, we get $\lambda=0$, whence (38).

Remark 7.8. In [Dr2], Drinfeld showed that conditions (36) and (32) can be removed from the definition of $\mathrm{GRT}_{1}(\mathbf{k})$ and $\mathfrak{g r t}_{1}(\mathbf{k})$, so $\mathrm{GRT}_{1}(\mathbf{k})=\underline{\mathbf{A s s o c}}_{0}(\mathbf{k})$. On the other hand, we can only prove $\operatorname{GRTM}_{(\overline{1}, 1)}(N, \mathbf{k}) \hookrightarrow \underline{\text { Pseudo }}_{(\overline{1}, 0)}(N, \mathbf{k})$. 
As we noticed, $\mathfrak{g t m}_{(\overline{1}, 1)}(N, \mathbf{k})$ is filtered by $\mathfrak{g}^{\geq n}=\{(\varphi, \psi) \mid \psi$ has degree $\geq n\}$.

Lemma 7.9. $\hat{\operatorname{rrgtm}}_{(\overline{1}, 1)}(N, \mathbf{k}) \subset \mathfrak{g r t m}_{(\overline{1}, 1)}(N, \mathbf{k})$.

Proof. Let $\Xi, \Theta, \eta(1), \ldots, \eta(N)$ be such that $e^{\Theta} e^{\eta(1)} \cdots e^{\eta(N)} e^{\Xi}=1$. Let $\bar{\psi} \in \mathfrak{g t m}_{1}(N, \mathbf{k})$ be of degree $\geq k$, and let $\psi$ be its degree $k$ component. Then for any $\alpha \in \mathbb{Z}$, we have

$$
\begin{aligned}
& \bar{\psi}(\Xi \mid \eta(\alpha), \ldots, \eta(\alpha+N-1))-\bar{\psi}(\Xi \mid \eta(\alpha+1), \ldots, \eta(\alpha+N)) \\
& =\operatorname{Ad}\left(e^{-\eta(\alpha)} \ldots e^{-\eta(1)}\right)\left(\bar{\psi}\left(\Theta \mid \eta^{\prime}(\alpha), \ldots, \eta^{\prime}(\alpha+1-N)\right)-\bar{\psi}\left(\Theta \mid \eta^{\prime}(\alpha+1), \ldots, \eta^{\prime}(\alpha+2-N)\right)\right)
\end{aligned}
$$

(we use the conventions of Lemma 6.6). Summing up these equalities for $\alpha=0, \ldots, N-1$, we get

$$
\begin{aligned}
& \left(1-\operatorname{Ad}\left(e^{\Xi}\right)\right) \bar{\psi}(\Xi \mid \eta(0), \ldots, \eta(N-1)) \\
& =\sum_{\alpha=1}^{N-1}\left(\operatorname{Ad}\left(e^{-\eta(\alpha)}\right)-1\right) \operatorname{Ad}\left(e^{-\eta(\alpha-1)} \cdots e^{-\eta(1)}\right) \bar{\psi}\left(\Theta \mid \eta^{\prime}(\alpha), \ldots, \eta^{\prime}(\alpha+1-N)\right) \\
& +\left(1-\operatorname{Ad}\left(e^{\Xi} e^{\eta(0)}\right)\right) \bar{\psi}\left(\Theta \mid \eta^{\prime}(0), \ldots, \eta^{\prime}(1-N)\right),
\end{aligned}
$$

which implies that $\psi$ satisfies (39). The other conditions satisfied by $\bar{\psi}$ imply that $\psi \in$ $\mathfrak{g r t m}_{(\overline{1}, 1)}(N, \mathbf{k})$.

7.4. The action of $\operatorname{GRTM}(N, \mathbf{k})$ on $\operatorname{Pseudo}(N, \mathbf{k}) \cdot \operatorname{GRTM}_{(\overline{1}, 1)}(N, \mathbf{k}) \operatorname{acts}$ on $\underline{\operatorname{Pseudo}}(N, \mathbf{k})$ from the right by $(\Phi, \Psi) *(h, k)=\left(\Phi^{\prime}, \Psi^{\prime}\right)$, where

$$
\Phi^{\prime}(U, V)=h(U, V) \Phi\left(U, \operatorname{Ad}(h(U, V))^{-1}(V)\right),
$$

$\Psi^{\prime}(A \mid b(0), \ldots, b(N-1))=k(A \mid b(0), \ldots, b(N-1))$

$\Psi\left(A \mid \operatorname{Ad}\left(k(A \mid b(0), \ldots, b(N-1))^{-1}\right)(b(0)), \ldots, \operatorname{Ad}\left(k(A \mid b(N-1), \ldots, b(2(N-1)))^{-1}\right)(b(N-1))\right)$.

This action preserves each $\underline{\mathbf{P s e u d o}}_{(a, \lambda)}(N, \mathbf{k})$, and it extends to an $\operatorname{action}$ of $\operatorname{GRTM}(N, \mathbf{k})$ on Pseudo $(N, \mathbf{k})$, which is compatible with the action of $(\mathbb{Z} / N \mathbb{Z})^{\times} \times \mathbf{k}^{\times}$on $(\mathbb{Z} / N \mathbb{Z}) \times \mathbf{k}$ and commutes with the left action of $\operatorname{GTM}(N, \mathbf{k})$ on $\underline{\mathbf{P s e u d o}}(N, \mathbf{k})$.

Theorem 7.10. Pseudo $(N, \mathbf{k})$ (resp., $\operatorname{Pseudo}_{\overline{1}}(N, \mathbf{k})$, $\operatorname{Pseudo}_{(\overline{1}, 1)}(N, \mathbf{k})$ ) is a torsor under $\operatorname{GRTM}(N, \mathbf{k})$ (resp., $\left.\operatorname{GRTM}_{\overline{1}}(N, \mathbf{k}), \operatorname{GRTM}_{(\overline{1}, 1)}(N, \mathbf{k})\right)$.

Proof. It suffices to prove that last statement. Let $\left(\Phi_{1}, \Psi_{1}\right)$ and $\left(\Phi_{2}, \Psi_{2}\right)$ be elements of $\operatorname{Pseudo}_{(\overline{1}, 1)}(N, \mathbf{k})$. Then there exists a unique pair $(h, k) \in \exp \left(\hat{\mathfrak{t}}_{3}^{0}\right) \times \exp \left(\hat{\mathfrak{t}}_{3, N}^{0}\right)$, such that $\left(\Phi_{1}, \Psi_{1}\right) *(h, k)=\left(\Phi_{2}, \Psi_{2}\right)$ (where the action is computed according to formulas (46), (47). We have to show that $(h, k) \in \operatorname{GRTM}_{(\overline{1}, 1)}(N, \mathbf{k})$.

Assume that $\left(\Phi_{1}, \Psi_{1}\right)$ and $\left(\Phi_{2}, \Psi_{2}\right)$ coincide up to order $\nu$ - 1, i.e., their difference belongs to $U\left(\mathfrak{t}_{3}^{0}\right)_{\geq \nu}^{\wedge} \oplus U\left(\mathfrak{t}_{3, N}^{0}\right)_{\geq \nu}^{\wedge}$ (the index means the grading where each generator has degree 1 and the ()$^{\wedge}$ means degree completion). Set $\varphi:=\left(\Phi_{2}-\Phi_{1} \bmod U\left(\mathfrak{t}_{3}^{0}\right)_{\geq \nu+1}^{\wedge}\right), \psi:=\left(\Psi_{2}-\Psi_{1} \bmod \right.$ $\left.U\left(\mathfrak{t}_{3, N}^{0}\right)_{\geq \nu+1}^{\wedge}\right)$. Since the $\Phi_{i}, \Psi_{i}$ are group-like and coincide up to order $n-1$, we have $\varphi \in\left(\mathfrak{t}_{3}^{0}\right)_{\nu}$ and $\psi \in\left(\mathfrak{t}_{3, N}^{0}\right)_{\nu}$. Let us show that $(\varphi, \psi) \in \mathfrak{g r t m}_{(\overline{1}, 1)}(N, \mathbf{k})$.

We first show that $\varphi \in \mathfrak{g r t}_{1}(\mathbf{k})$. Comparing identities (6) and (7) satisfied by $\Phi_{1}$ and $\Phi_{2}$, we find that $\varphi$ satisfies (35), (37). According to [Dr2], Proposition 5.7, this implies that $\varphi$ also satifies (36) and therefore belongs to $\mathfrak{g r t}_{1}(\mathbf{k})$. 
Comparing now identities (8) and (9) satisfied by $\left(\Phi_{1}, \Psi_{1}\right)$ and $\left(\Phi_{2}, \Psi_{2}\right)$, we find that $\psi$ satisfies (38) and $(\varphi, \psi)$ satisfies (40). Let us show that $\psi$ also satisfies (39). If $\Psi(A \mid b(0), \ldots, b(N-$ 1)) satisfies (8), we have

$$
X(0) \cdots X(N-1)=1
$$

where

$$
\begin{aligned}
& X(a):=\Psi(A \mid b(a), \ldots, b(a+N-1))^{-1} e^{N b(a) / 2} \Psi(C \mid b(a), \ldots, b(a+1-N)) e^{C} \\
& \Psi(C \mid b(a), \ldots, b(a+1-N))^{-1} e^{N b(a) / 2} \Psi(A \mid b(a), \ldots, b(a+N-1)) e^{A} .
\end{aligned}
$$

Let $X_{i}(a)$ be the r.h.s. of (49) for $\Psi=\Psi_{i}(i=1,2)$. Then

$$
\begin{aligned}
& X_{2}(a)=X_{1}(a)+[\psi(A \mid b(A), \ldots, b(a+N-1))-\psi(C \mid b(a), \ldots, b(a+1-N)), A] \\
& -N[\psi(C \mid b(a), \ldots, b(a+1-N)), b(a)]+\text { terms of degree }>\nu+1,
\end{aligned}
$$

and $X_{i}(a)=1+$ terms of degree $>0$.

Therefore

(l.h.s. of (48) for $\left.\Psi_{2}\right)$ - (l.h.s. of (48) for $\left.\Psi_{1}\right)$

$$
\begin{aligned}
& =\sum_{a=0}^{N-1}([\psi(A \mid b(a), \ldots, b(a+N-1))-\psi(C \mid b(a), \ldots, b(a+1-N)), A] \\
& -N[\psi(C \mid b(a), \ldots, b(a+1-N)), b(a)])+ \text { terms of degree }>\nu+1 .
\end{aligned}
$$

Since $\Psi_{1}$ and $\Psi_{2}$ satisfy (48), we get

$$
\begin{aligned}
& \sum_{a=0}^{N-1}([\psi(A \mid b(a), \ldots, b(a+N-1))-\psi(C \mid b(a), \ldots, b(a+1-N)), A] \\
& -N[\psi(C \mid b(a), \ldots, b(a+1-N)), b(a)])=0 .
\end{aligned}
$$

Since $\psi$ satisfies (38), this identity is equivalent to

$$
\begin{aligned}
& {[\psi(A \mid b(0), \ldots, b(N-1))-\psi(C \mid b(0), \ldots, b(1-N)), A]} \\
& -\sum_{a=0}^{N-1}[\psi(C \mid b(a), \ldots, b(a+1-N)), b(a)]=0,
\end{aligned}
$$

which is equivalent to (39), after the change of variables $A \mapsto C, C \mapsto A, b(a) \mapsto b(-a)$.

Hence $(\varphi, \psi) \in \mathfrak{g r t m}_{(\overline{1}, 1)}(N, \mathbf{k})$. Set $\left(\Phi_{1}^{\prime}, \Psi_{1}^{\prime}\right):=\left(\Phi_{1}, \Psi_{1}\right) * \operatorname{Exp}(\varphi, \psi)$, where $\operatorname{Exp}: \mathfrak{g r t m}_{(\overline{1}, 1)}(N, \mathbf{k}) \rightarrow$ $\operatorname{GRTM}_{(\overline{1}, 1)}(N, \mathbf{k})$ is the exponential map for the group structure of $\operatorname{GRTM}_{(\overline{1}, 1)}(N, \mathbf{k})$ (it is a restriction of the exponential of $\left.\operatorname{Mag}_{(\overline{1}, 1)}(N, \mathbf{k})\right)$. Then $\left(\Phi_{1}^{\prime}, \Psi_{1}^{\prime}\right) \in \operatorname{Pseudo}_{(\overline{1}, 1)}(N, \mathbf{k})$ coincides with $\left(\Phi_{2}, \Psi_{2}\right)$ up to order $\nu$. Using successive approximations, we then construct $(h, k) \in \operatorname{GRTM}_{(\overline{1}, 1)}(N, \mathbf{k})$ such that $\left(\Phi_{1}, \Psi_{1}\right) *(h, k)=\left(\Phi_{2}, \Psi_{2}\right)$.

As in [Dr2], we get:

Corollary 7.11. Any $(\Phi, \Psi) \in \mathbf{P} \operatorname{seudo}(N, \mathbf{k})$ gives rise to a group isomorphism $s_{(\Phi, \Psi)}$ : $\operatorname{GRTM}(N, \mathbf{k}) \rightarrow \operatorname{GTM}(N, \mathbf{k})$, defined by the condition that $(\Phi, \Psi) * g=s_{(\Phi, \Psi)}(g) *(\Phi, \Psi)$. The diagram

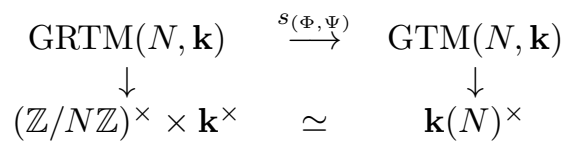

is commutative, so $s_{(\Phi, \Psi)}\left(\operatorname{GRTM}_{(\overline{1}, 1)}(N, \mathbf{k})\right)=\operatorname{GTM}_{(\overline{1}, 1)}(N, \mathbf{k})$. The resulting isomorphism $\mathfrak{g r t m}_{\text {bar1,1) }}(N, \mathbf{k}) \rightarrow \mathfrak{g t m}_{\text {bar1,1) }}(N, \mathbf{k})$ is filtered, and the inverse of the associated graded isomorphism coincides with the map defined in Lemma 7.9. The splitting of the exact sequence 
(29) corresponding to $(\Phi, \Psi)$ is the morphism $\operatorname{Lie}\left(s_{(\Phi, \Psi)}\right) \circ i: \mathbf{k} \rightarrow \mathfrak{g t m}_{\overline{1}}(N, \mathbf{k})$, where $i$ is the canonical embedding $\mathbf{k} \rightarrow \mathfrak{g r t m}_{\overline{1}}(N, \mathbf{k})$.

7.5. $\underline{\text { Pseudo }}(N, \mathbf{k})$ when $N=1$.

Lemma 7.12. There is a unique isomorphism $\underline{\mathbf{A s s o c}}(\mathbf{k}) \times \mathbf{k} \rightarrow \underline{\text { Pseudo }}(1, \mathbf{k})$, taking $((\lambda, \Phi), \gamma)$ to $(\overline{1}, \lambda, \Phi, \Psi)$, where $\Psi=e^{\gamma t^{23}} \Phi$.

Proof. Let $(\overline{1}, \lambda, \Phi, \Psi)$ belong to $\underline{\text { Pseudo }}(1, \mathbf{k})$. Let $\varepsilon^{(0)}: \mathfrak{t}_{3} \rightarrow \mathfrak{t}_{2}$ be the Lie algebra morphism $t^{0 i} \mapsto 0, t^{12} \mapsto t^{12}$. Then there exists $\gamma \in \mathbf{k}$ such that $\varepsilon^{(0)}(\Psi)=e^{\gamma t^{12}}$. Applying $\varepsilon^{(0)}$ to (9) and taking into account that $\Phi, \Psi$ are group-like, we get $\Psi=e^{\gamma t^{12}} \Phi$. Conversely, if $\Phi \in \underline{\mathbf{A s s o c}}_{\lambda}(\mathbf{k})$ and $\gamma \in \mathbf{k}$, then $\left(\Phi, e^{\gamma t^{23}} \Phi\right)$ belongs to $\underline{\text { Pseudo }}_{(\overline{1}, \lambda)}(\mathbf{k})$.

Lemma 7.13. There exists a unique isomorphism $\mathfrak{g r t m}_{(\overline{1}, 1)}(1, \mathbf{k}) \simeq \mathfrak{g r t}_{(\overline{1}, 1)}(\mathbf{k}) \oplus \mathbf{k}\left(0, t^{12}\right)$, where $\left(0, t^{12}\right)$ is central and has degree 1.

Proof. Similarly to the previous lemma, one proves that $(\varphi, \psi) \in \mathfrak{g r t m}_{(\overline{1}, 1)}(1, \mathbf{k})$ iff $\varphi \in$ $\mathfrak{g r t}_{(\overline{1}, 1)}(\mathbf{k})$ and there exists $\gamma \in \mathbf{k}$ such that $\psi=\varphi+\gamma t^{12}$. One then checks that $\left(0, t^{12}\right)$ is central.

Remark 7.14. $\mathfrak{g r t m}_{(\overline{1}, 1)}(N, \mathbf{k})$ in degree 1 . One checks that the degree 1 part of $\mathfrak{g r t m}_{(\overline{1}, 1)}(N, \mathbf{k})$ is spanned by the $(0, b(a)+b(-a))$, where $a \in(\mathbb{Z} / N \mathbb{Z}) /\{ \pm 1\}$. Moreover, $(0, b(0))$ is central in $\mathfrak{g r t m}_{(\overline{1}, 1)}(N, \mathbf{k})$.

\section{The EQUALity GTK $=\widehat{\mathrm{GT}}$}

Recall that $\varphi_{N}: F_{2} \rightarrow \mathbb{Z} / N \mathbb{Z}$ is the morphism $x \mapsto \overline{1}, y \mapsto \overline{0}$ and that $\operatorname{Ker} \varphi_{N} \subset F_{2}$ is freely generated by $X, y(0), \ldots, y(N-1)$, where $X:=x^{N}, y(\alpha):=x^{\alpha} y x^{-\alpha}$. Define a group morphism $\delta_{N}: \operatorname{Ker} \varphi_{N} \rightarrow F_{2}$ by $X \mapsto x, y(0) \mapsto y, y(\alpha) \mapsto 1$ for $\alpha \in[1, N-1]$. Define also a group morphism $c_{N}: \operatorname{Ker} \varphi_{N} \rightarrow \mathbb{Z}$ by $c_{N}:=c \circ \delta_{N}$, where $c: F_{2} \rightarrow \mathbb{Z}$ is $x \mapsto 0, y \mapsto 1$.

Recall that for any $(\lambda, f) \in \underline{\widehat{\mathrm{GT}}}, f \in \widehat{F}_{2}^{\prime} \subset\left(\operatorname{Ker} \varphi_{N}\right)^{\wedge}$. In [Ih2], Ihara defined GTK $\subset \widehat{\mathrm{GT}}$ as the set of $(\lambda, f)$, such that

$$
\forall N \geq 1, \delta_{N}(f)=y^{c_{N}(f)} f .
$$

Theorem 8.1. GTK $=\widehat{\mathrm{GT}}$; more generally, if $(\lambda, f) \in \widehat{\mathrm{GT}}$, then (50) holds.

Proof. Set $W_{n}:=W_{n, 1}=\left\{\left(Z_{1}, \ldots, Z_{n}\right) \in\left(\mathbb{C}^{\times}\right)^{n} \mid \forall i \neq j, Z_{i} \neq Z_{j}\right\}$ and recall that $P_{n+1}=$ $\pi_{1}\left(W_{n}, b\right)$ (see Subsection 1.3). The morphism $\varphi_{n, N}: P_{n+1} \rightarrow(\mathbb{Z} / N \mathbb{Z})^{n}$ is induced by the covering $W_{n, N} \rightarrow W_{n},\left(z_{1}, \ldots, z_{n}\right) \mapsto\left(z_{1}^{N}, \ldots, z_{n}^{N}\right)$, where $W_{n, N}=\left\{\left(z_{1}, \ldots, z_{n}\right) \in\left(\mathbb{C}^{\times}\right)^{n} \mid \forall i \neq\right.$ $\left.j, \forall \zeta \in \mu_{N}(\mathbb{C}), z_{i} \neq \zeta z_{j}\right\}$. Hence $\operatorname{Ker} \varphi_{n, N}=\pi_{1}\left(W_{n, N}, b\right)$ (it is denoted $\left.K_{n, N}\right)$. In addition to the inclusion $K_{n, N} \hookrightarrow P_{n+1}$, we have a morphism $\delta_{N}: K_{n, N} \rightarrow P_{n+1}$, induced by $W_{n, N} \rightarrow W_{n}$, $\left(z_{1}, \ldots, z_{n}\right) \mapsto\left(z_{1}, \ldots, z_{n}\right)$ by taking fundamental groups.

The morphisms $\partial_{i}: P_{n} \rightarrow P_{n+1}(i=0, \ldots, n+1)$ are given (in terms of the Artin generators $x_{j k}, 0 \leq j<k \leq n-1$ of $\left.P_{n}\right)$ by $\partial_{0}: x_{j k} \mapsto x_{j+1, k+1}, \partial_{n+1}: x_{j k} \mapsto x_{j k}$ and $\partial_{i}: x_{j k} \mapsto x_{j k}$ if $k<i-1, x_{j k} \mapsto x_{j, k+1}$ if $k>i-1>j, x_{j k} \mapsto x_{j+1, k+1}$ if $j>i-1, x_{j, i-1} \mapsto x_{j, i-1} x_{j i}$ if $j<i-1, x_{i-1, j} \mapsto x_{i-1, j+1} x_{i, j+1}$ if $j>i-1$.

Lemma 8.2. There exist morphisms $\tilde{\partial}_{i}: P_{n} \rightarrow P_{n+1}(i=1, \ldots, n)$ given by the same formulas, except $\tilde{\partial}_{i}: x_{0, i-1} \mapsto x_{0, i-1} x_{0, i} x_{i-1, i}$ if $i=2, \ldots, n$ and $\tilde{\partial}_{1}: x_{01} \mapsto x_{01} x_{02} x_{12}$.

Proof of Lemma. One can show that for $i=1, \ldots, n$, the morphism $\partial_{i}: P_{n} \rightarrow P_{n+1}$ is induced by the sequence of maps $W_{n-1} \supset W_{n-1}^{i, \varepsilon} \rightarrow W_{n}$, where $\left.\varepsilon \in\right] 0,1[$, and: (a) for $i=2, \ldots, n$, $W_{n-1}^{i, \varepsilon}:=\left\{\left(z_{1}, \ldots, z_{n-1}\right) \in W_{n-1}|\forall j \neq i,| z_{j} / z_{i-1}-1 \mid>\varepsilon\right\}$ and $W_{n-1}^{i, \varepsilon} \rightarrow W_{n}$ is $\left(z_{1}, \ldots, z_{n-1}\right) \mapsto$ 
$\left(z_{1}, \ldots, z_{i-1}, z_{i-1}+\varepsilon\left|z_{i-1}\right|, z_{i}, \ldots, z_{n-1}\right)$, and (b) for $i=1, W_{n-1}^{1, \varepsilon}=\left\{\left(z_{1}, \ldots, z_{n}\right) \in W_{n}|\forall i,| z_{i} / z_{1} \mid>\right.$ $\varepsilon\}$ and $W_{n-1}^{1, \varepsilon} \rightarrow W_{n}$ is $\left(z_{1}, \ldots, z_{n-1}\right) \mapsto\left(\varepsilon\left|z_{1}\right|, z_{1}, \ldots, z_{n-1}\right)$.

$\tilde{\partial}_{i}: P_{n} \rightarrow P_{n+1}(i=1, \ldots, n)$ is then defined as the morphism induced by the sequence of maps $W_{n-1} \supset W_{n-1}^{i, \varepsilon} \rightarrow W_{n}$, where $W_{n-1}^{i, \varepsilon} \rightarrow W_{n}$ is $\left(z_{1}, \ldots, z_{n-1}\right) \mapsto\left(z_{1}, \ldots, z_{i-1}, z_{i-1}(1+\right.$ $\left.\varepsilon), z_{i}, \ldots, z_{n-1}\right)$ for $i=2, \ldots, n$ and $\left(z_{1}, \ldots, z_{n-1}\right) \mapsto\left(\varepsilon z_{1}, z_{1}, \ldots, z_{n-1}\right)$ for $i=1$. One checks that the additional possible winding of $i$ around $i+1$ corresponds to the above explicit formulas for $\tilde{\partial}_{i}$.

On the other hand, $\partial_{0}: P_{n} \rightarrow P_{n+1}$ is induced by the inclusion $\left\{\left(z_{1}, \ldots, z_{n}\right) \mid \forall i, z_{i} \notin \mathbb{R}_{-}\right.$ and $\left.\forall i \neq j, z_{i} \neq z_{j}\right\} \subset W_{n}$ and $\partial_{n+1}: P_{n} \rightarrow P_{n+1}$ is induced by the sequence of maps $W_{n-1} \supset W_{n-1} \cap \mathbf{D}^{n-1} \rightarrow W_{n}$, where $\mathbf{D}=\{z \in \mathbb{C}|| z \mid<1\}$ and $W_{n-1} \cap \mathbf{D}^{n-1} \rightarrow W_{n}$ is $\left(z_{1}, \ldots, z_{n-1}\right) \mapsto\left(z_{1}, \ldots, z_{n-1}, 1\right)$.

Lemma 8.3. The morphisms $\tilde{\partial}_{1}, \ldots, \tilde{\partial}_{n}, \partial_{n+1}$ restrict to morphisms $K_{n-1, N} \rightarrow K_{n, N}$, and $\partial_{0}$ factors as $P_{n} \rightarrow K_{n, N} \subset P_{n+1}$.

Proof. Recall that $\varphi_{n, N}: P_{n+1} \rightarrow(\mathbb{Z} / N \mathbb{Z})^{n}$ is given by $x_{0 i} \mapsto s_{i}$, the $i$ th generator of $P_{n} \stackrel{\varphi_{n-1, N}}{\longrightarrow}(\mathbb{Z} / N \mathbb{Z})^{n-1}$

$(\mathbb{Z} / N \mathbb{Z})^{n}$. We have commutative diagrams $\tilde{\partial}_{i}, \partial_{n+1} \downarrow \quad \downarrow \tilde{\alpha}_{i}, \alpha_{n+1}$, where for $i=2, \ldots, n$,

$$
P_{n+1} \stackrel{\varphi_{n}, N}{\rightarrow} \quad(\mathbb{Z} / N \mathbb{Z})^{n}
$$

$\tilde{\alpha}_{i}\left(s_{j}\right)=s_{j}$ for $j<i-1, \tilde{\alpha}_{i}\left(s_{j}\right)=s_{j+1}$ for $j>i-1, \tilde{\alpha}_{i}\left(s_{i-1}\right)=s_{i-1}+s_{i}, \tilde{\alpha}_{1}=\tilde{\alpha}_{2}$, and $\alpha_{n+1}\left(s_{j}\right)=s_{j}$ for any $j$. On the other hand, $\varphi_{n, N} \circ \partial_{0}=0$. This implies the result.

In view of the topological interpretation of $\partial_{0}, \tilde{\partial}_{1, \ldots, n}, \partial_{n+1}$, the morphisms $\tilde{\partial}_{1, \ldots, n}, \partial_{n+1}$ : $K_{n-1, N} \rightarrow K_{n, N}$ and $\partial_{0}: P_{n} \rightarrow K_{n-1, N}$ constructed in Lemma 8.3 can be interpreted as induced by the following sequences of maps: (a) in the case of $\left(\tilde{\partial}_{i}\right)_{i=2, \ldots, n}$, the maps ${ }^{6} W_{n-1, N} \supset$ $W_{n-1, N}^{i, \varepsilon} \rightarrow W_{n, N}, W_{n-1, N}^{i, \varepsilon}:=\left\{\left(z_{1}, \ldots, z_{n}\right) \in W_{n-1, N}|\forall j \neq i-1,|\left(z_{j} / z_{i-1}\right)^{N}-1 \mid>\varepsilon\right\}$ and $W_{n-1, N}^{i, \varepsilon} \rightarrow W_{n, N}$ is $\left(z_{1}, \ldots, z_{n-1}\right) \mapsto\left(z_{1}, \ldots, z_{i-1}(1+\varepsilon)^{1 / N}, \ldots, z_{n-1}\right)$; (b) in the case of $\tilde{\partial}_{1}$, the maps $W_{n-1, N} \supset W_{n-1, N}^{1, \varepsilon} \rightarrow W_{n, N}$, where $0<\varepsilon<1, W_{n-1, N}^{1, \varepsilon}=\left\{\left(z_{1}, \ldots, z_{n-1}\right) \in W_{n-1, N} \mid \forall j \neq\right.$ $\left.1,\left|\left(z_{j} / z_{1}\right)^{N}\right|>\varepsilon\right\}$ and $W_{n-1, N}^{1, \varepsilon} \rightarrow W_{n, N}$ is $\left(z_{1}, \ldots, z_{n-1}\right) \mapsto\left(\varepsilon^{1 / N} z_{1}, z_{1}, \ldots, z_{n-1}\right) ;$ (c) in the case of $\partial_{n+1}$, the maps $W_{n-1, N} \supset W_{n-1, N} \cap \mathbf{D}^{n-1} \rightarrow W_{n, N}$, where $W_{n-1, N} \cap \mathbf{D}^{n-1} \rightarrow W_{n, N}$ is $\left(z_{1}, \ldots, z_{n-1}\right) \mapsto\left(z_{1}, \ldots, z_{n-1}, 1\right)$; (d) in the case of $\partial_{0}$, the map $\left\{\left(z_{1}, \ldots, z_{n}\right) \in W_{n} \mid \forall i, \arg \left(z_{i}\right)<\right.$ $\pi / n\} \rightarrow W_{n, N},\left(z_{1}, \ldots, z_{n}\right) \mapsto\left(z_{1}, \ldots, z_{n}\right)$.

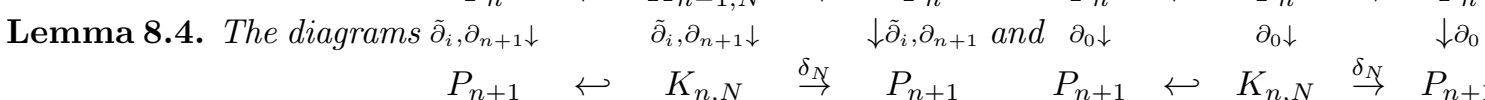

commute.

$$
P_{n+1} \hookleftarrow K_{n, N} \quad \stackrel{\delta_{N}}{\rightarrow} P_{n+1} \quad P_{n+1} \hookleftarrow K_{n, N} \stackrel{\delta_{N}}{\rightarrow} P_{n+1}
$$

Proof. We have already seen that the left part of each diagram commutes, so it remains to show the right parts. For this, we rely on the above topological interpretations.

Let $i \in\{1, \ldots, n\}$. If $i=2, \ldots, n$, let $\varepsilon>0$ be such that $\left\{u \in \mathbb{C} \| u^{N}-1 \mid<\varepsilon\right\}$ consists of disjoint contractible neighborhoods of the $\zeta \in \mu_{N}(\mathbb{C})$; and let $0<\varepsilon<1$ if $i=1$; then the diagram

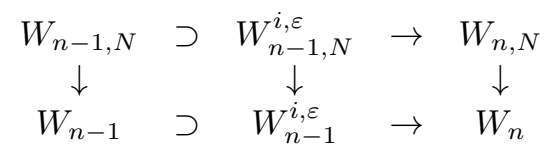

\footnotetext{
${ }^{6}$ Here $\varepsilon>0$ is small enough for $\left\{u \in \mathbb{C}|| u^{N}-1 \mid<\varepsilon\right\}$ to consist of disjoint contractible neighborhoods of the $\zeta \in \mu_{N}(\mathbb{C})$ in $\mathbb{C}^{\times}$.
} 
commutes, where the vertical maps are all restrictions of $\left(z_{1}, \ldots, z_{k}\right) \mapsto\left(z_{1}^{N}, \ldots, z_{k}^{N}\right)(k=n-1, n)$. Passing to fundamental groups, we see that the right diagram involving $\tilde{\partial}_{i}$ commutes.

When $i=n+1$, the diagram

$$
\begin{array}{cccccc}
W_{n-1, N} & \supset & W_{n-1, N} \cap \mathbf{D}^{n-1} & \rightarrow & W_{n, N} \\
\downarrow & & \downarrow & & & \downarrow \\
W_{n-1} & \supset & W_{n-1} \cap \mathbf{D}^{n-1} & \rightarrow & W_{n}
\end{array}
$$

commutes, where the vertical maps are as above. This implies the wanted result involving $\partial_{n+1}$.

The commutativity of the diagram involving $\partial_{0}$ similarly follows from that of

$\begin{array}{rlc}\left\{\left(z_{1}, \ldots, z_{n}\right) \in W_{n} \mid \forall i, \arg \left(z_{i}\right)<\pi / n\right\} & \rightarrow & W_{n, N} \\ \downarrow & & \downarrow \\ \left\{\left(Z_{1}, \ldots, Z_{n}\right) \in W_{n} \mid \forall i, Z_{i} \notin \mathbb{R}_{-}\right\} & \rightarrow & W_{n}\end{array}$.

Lemma 8.5. Let $f \in \widehat{F}_{2}^{\prime}$. Then for $i=1,2,3, \tilde{\partial}_{i}(f)=\partial_{i}(f)$.

Proof. For $f \in \widehat{F}_{2}$, recall that $c(f) \in \widehat{\mathbb{Z}}$ is defined by $f(1, y)=y^{c(f)}$ and define $b(f) \in \widehat{\mathbb{Z}}$ by $f(x, 1)=x^{b(f)}$. As $\tilde{\partial}_{1}(f)=f\left(x_{01} x_{02} x_{12}, x_{23}\right)$ and since $x_{01}$ commutes with both $x_{02} x_{12}$ and $x_{23}$, we get $\tilde{\partial}_{1}(f)=x_{01}^{b(f)} \partial_{1}(f)$. Similarly, $\tilde{\partial}_{2}(f)=f\left(x_{01} x_{02} x_{12}, x_{13} x_{23}\right)$, and since $x_{12}$ commutes with both $x_{01} x_{02}$ and $x_{13} x_{23}$, we get $\tilde{\partial}_{2}(f)=x_{12}^{b(f)} \partial_{2}(f)$; and $\tilde{\partial}_{3}(f)=f\left(x_{01}, x_{13} x_{23}\right)$ implies $\tilde{\partial}_{3}(f)=\partial_{3}(f)$. If $f \in \widehat{F}_{2}^{\prime}$, then $b(f)=c(f)=0$, which implies the result.

Let $(\lambda, f) \in \widehat{\widehat{\mathrm{GT}}}$. Then $f \in \widehat{F}_{2}^{\prime}$, hence

$$
\partial_{0}(f) \tilde{\partial}_{2}(f) \partial_{4}(f)=\tilde{\partial}_{3}(f) \tilde{\partial}_{1}(f) .
$$

$\partial_{0}(f)$ belongs to $K_{4, N}$, and since $f \in\left(\operatorname{Ker} \varphi_{N}\right)^{\wedge} \subset \widehat{K}_{3, N}$, so do $\tilde{\partial}_{1,2,3}(f)$ and $\partial_{4}(f)$. So $(51)$ takes place in $\widehat{K}_{3, N}$. Let us apply $\delta_{N}$ to this identity. In view of the above commutation relations, we get $\partial_{0}(f) \tilde{\partial}_{2}(h) \partial_{4}(h)=\tilde{\partial}_{3}(h) \tilde{\partial}_{1}(h)$, where $h=\delta_{N}(g)$; this equality takes place in $\widehat{P}_{4}$. This is rewritten $\partial_{0}(f) x_{12}^{b(h)} \partial_{2}(h) \partial_{4}(h)=\partial_{3}(h) x_{01}^{b(h)} \partial_{1}(h)$. For a general $k \in \operatorname{Ker} \varphi_{N}$, we have $b(k)=N b\left(\delta_{N}(k)\right)$ so $N b(h)=b(f)=0$ as $f \in \widehat{F}_{2}^{\prime}$, so $b(h)=0$. Hence

$$
\partial_{0}(f) \partial_{2}(h) \partial_{4}(h)=\partial_{3}(h) \partial_{1}(h) .
$$

Applying now the morphism $\varepsilon_{0}: \widehat{P}_{4} \rightarrow \widehat{P}_{3}$ of erasing of the 0th strand (i.e., $x_{0 i} \mapsto 1, x_{i j} \mapsto x_{i j}$ for $1 \leq i<j \leq 3)$, and using the fact that $\varepsilon_{0} \circ \partial_{0}=\varepsilon_{0} \circ \partial_{1}=\mathrm{id}, \varepsilon_{0} \circ \partial_{2}(h)=\left(x_{13} x_{23}\right)^{c(h)}$, $\varepsilon_{0} \circ \partial_{3}(h)=\left(x_{12} x_{13}\right)^{c(h)}, \varepsilon_{0} \circ \partial_{4}(h)=x_{12}^{c(h)}$ for $h \in \widehat{F}_{2}$, we get $f \cdot\left(x_{13} x_{23}\right)^{c(h)} \cdot x_{12}^{c(h)}=$ $\left(x_{12} x_{13}\right)^{c(h)} \cdot h$, hence $h=\left(x_{12} x_{13}\right)^{-c(h)} \cdot f \cdot\left(x_{13} x_{23}\right)^{c(h)} x_{12}^{c(h)}=x_{23}^{c(h)} f$ as $x_{12} x_{13} x_{23}$ is central in $\widehat{P}_{3}$, i.e., $h(x, y)=y^{c(h)} f(x, y)$, as wanted.

Remark 8.6. It is observed in [Ih2] that Theorem 8.1 implies that $c_{N_{1} N_{2}}(f)=c_{N_{1}}(f)+$ $c_{N_{2}}(f)$ for $(\lambda, f) \in \widehat{\mathrm{GT}}$, and $N_{1}, N_{2} \geq 1$; indeed, $y^{c_{N_{1} N_{2}}(f)} f=\delta_{N_{1} N_{2}}(f)=\delta_{N_{1}}\left(\delta_{N_{2}}(f)\right)=$ $\delta_{N_{1}}\left(y^{c_{N_{2}}(f)} f\right)=y^{c_{N_{1}}(f)+c_{N_{2}}(f)} f$.

Remark 8.7. The analogue of Theorem 8.1 for $\underline{G T}_{l}$ can be formulated as follows. For $\nu \geq 0$, one can show that $\operatorname{Ker}\left[\left(F_{2}\right)_{l} \stackrel{\left(\varphi_{l}^{\nu}\right)_{l}}{\longrightarrow} \mathbb{Z} / l^{\nu} \mathbb{Z}\right]=\left(\operatorname{Ker} \varphi_{l^{\nu}}\right)_{l}\left(\right.$ if $N \geq 1$, then $\left(\varphi_{N}\right)_{l}=\left(\varphi_{l^{\nu}}\right)_{l}$, where $\nu$ is the $l$-adic valuation of $N)$; as $\operatorname{Ker} \varphi_{l^{\nu}}$ is freely generated by $X:=x^{l^{\nu}}$ and $y(\alpha):=x^{\alpha} y x^{-\alpha}$, $\alpha \in\left[0, l^{\nu}-1\right]$, we have a morphism $\delta_{l^{\nu}}:\left(\operatorname{Ker} \varphi_{l^{\nu}}\right)_{l} \rightarrow\left(F_{2}\right)_{l}, X \mapsto x, y(0) \mapsto y, y(\alpha) \mapsto 1$, $\alpha \in\left[1, l^{\nu}-1\right]$. We then define $c_{l^{\nu}}:\left(\operatorname{Ker} \varphi_{l^{\nu}}\right)_{l} \rightarrow \mathbb{Z}_{l}$ as $c_{l^{\nu}}:=c \circ \delta_{l^{\nu}}$, where $c:\left(F_{2}\right)_{l} \rightarrow \mathbb{Z}_{l}$ is the morphism given by $x \mapsto 1, y \mapsto 0$. The analogue of Theorem 8.1 then says that for any $(\lambda, f) \in \underline{\mathrm{GT}}_{l}$, we have $\delta_{l^{\nu}}(f)=y^{c_{l \nu}(f)} f$, which implies that for any $(\lambda, f) \in \underline{\mathrm{GT}}_{l}$, we have $c_{l^{\nu}}(f)=\nu c_{l}(f)$. 


\section{The distribution subgroup $\operatorname{Gtmd}(N, \mathbf{k}) \subset \operatorname{GTM}(N, \mathbf{k})$}

9.1. The endomorphisms $\delta_{N}$ of $\widehat{\mathrm{GTM}}$. For $\underline{f}=(\lambda, f) \in \widehat{\mathrm{GT}}$, set $\chi(f):=\lambda ; \chi: \widehat{\mathrm{GT}} \rightarrow$ $\widehat{\mathbb{Z}}^{\times}$is the cyclotomic character. Set $c_{N}(\underline{f}):=c_{N}(f)$. Then $c_{N}$ satisfies the cocycle identity $c_{N}\left(\underline{f}_{1} \underline{f}_{2}\right)=c_{N}\left(\underline{f}_{1}\right) \chi\left(\underline{f}_{2}\right)+c_{N}\left(\underline{f}_{2}\right)$ (more generally, this identity holds for $\underline{f}_{i} \in \widehat{\mathrm{GT}}$, if $\chi\left(\underline{f}_{2}\right) \bmod$ $N$ is in $\left.(\mathbb{Z} / N \mathbb{Z})^{\times}\right)$. It follows that we have a group endomorphism $\delta_{N}$ of $\widehat{\mathrm{GT}} \ltimes \widehat{\mathbb{Z}}, \delta_{N}(\underline{f}, s):=$ $\left(\underline{f}, s+c_{N}(\underline{f})\right)$.

According to Theorem 8.1, this endomorphism corresponds, under the isomorphism $\widehat{\mathrm{GTM}} \simeq$ $\widehat{\mathrm{GT}} \ltimes \widehat{\mathbb{Z}}$ (Proposition 5.4), to the self-map $(\lambda, \lambda, f, g) \mapsto\left(\lambda, \lambda, f, \delta_{N}(g)\right)$ of GTM. (According to Proposition 5.4, $g$ has the form $y^{s} f$, so since $f \in \widehat{F}_{2}^{\prime}, g \in \operatorname{Ker} \hat{b} \subset\left(\operatorname{Ker} \varphi_{N}\right)^{\wedge}$, where $\widehat{\mathrm{GT}} \rightarrow \widehat{\mathrm{GTM}}$ $g(x, 1)=x^{\hat{b}(g)}$, so $\delta_{N}(g)$ is well-defined.) Of course, the diagram $\searrow \frac{\downarrow \delta_{N}}{\mathrm{GTM}}$ commutes.

9.2. The morphisms $\delta_{N N^{\prime}}: \underline{\operatorname{GTM}}(N)_{l} \rightarrow \underline{\operatorname{GTM}}\left(N^{\prime}\right)_{l}$. Let us assume that $N^{\prime} \mid N$; we set $d:=N / N^{\prime}$. In this subsection, we construct morphisms $\delta_{N N^{\prime}}: \underline{\operatorname{GTM}}(N)_{l} \rightarrow \underline{\operatorname{GTM}}\left(N^{\prime}\right)_{l}$, such $\widehat{\underline{\mathrm{GTM}}} \rightarrow \underline{\mathrm{GTM}}(N)_{l}$

that $\frac{\delta_{d} \downarrow}{\downarrow} \quad \downarrow \delta_{N N^{\prime}} \quad$ commutes.

$\widehat{\mathrm{GTM}} \rightarrow \underline{\operatorname{GTM}}\left(N^{\prime}\right)_{l}$

We first define $\frac{\delta_{N N^{\prime}}}{:} K_{n, N} \rightarrow K_{n, N^{\prime}}$ to be the group morphism induced by the map $W_{n, N} \rightarrow W_{n, N^{\prime}},\left(z_{1}, \ldots, z_{n}\right) \mapsto\left(z_{1}^{d}, \ldots, z_{n}^{d}\right)$ by taking fundamental groups. One checks that this map restricts to $\delta_{N N^{\prime}}: \operatorname{Ker} \varphi_{N} \rightarrow \operatorname{Ker} \varphi_{N^{\prime}}$ under $\operatorname{Ker} \varphi_{\nu} \subset K_{2, \nu}\left(\nu=N, N^{\prime}\right)$.

Recall the ring morphism [[-]]: $\mathbb{Z}_{l}(N) \rightarrow \mathbb{Z}_{l}\left(N^{\prime}\right)$ given by $(a, r) \mapsto(\overline{\bar{a}}, \tilde{\tilde{a}}+d r)$, where $a \mapsto \overline{\bar{a}}$ is the morphism $\mathbb{Z} / N \mathbb{Z} \rightarrow \mathbb{Z} / d \mathbb{Z}, \overline{1} \mapsto \overline{1}, \tilde{\tilde{a}} \in\left[0, N^{\prime}-1\right]$ is the integral part of $\tilde{a} / d$, where $\tilde{a} \in[0, N-1]$ is the lift of $a$; here $d=N / N^{\prime}$.

Let $(\lambda, \mu, f, g) \in \underline{\operatorname{GTM}}(N)_{l}$. Recall that $(\lambda, \mu, f, g) \in\left(2 \mathbb{Z}_{l}+1\right) \times \mathbb{Z}_{l}(N) \times\left(F_{2}\right)_{l} \times\left(\operatorname{Ker} \varphi_{N}\right)_{l}$. We then set $\delta_{N N^{\prime}}(\lambda, \mu, f, g):=\left(\lambda,[[\mu]], f, \delta_{N N^{\prime}}(g)\right)$.

Proposition 9.1. This defines a map $\delta_{N N^{\prime}}: \underline{\operatorname{GTM}}(N)_{l} \rightarrow \underline{\operatorname{GTM}}\left(N^{\prime}\right)_{l}$, such that $\delta_{N N^{\prime}}\left(\underline{g}_{1} \underline{g}_{2}\right)=$ $\delta_{N N^{\prime}}\left(\underline{g}_{1}\right) \delta_{N N^{\prime}}\left(\underline{g}_{2}\right)$ for $\underline{g}_{2} \in \underline{\mathrm{GTM}}(N)_{l} \times_{\mathbb{Z} / d \mathbb{Z}}(\mathbb{Z} / d \mathbb{Z})^{\times}$, which restricts to a group morphism $\delta_{N N^{\prime}}: \operatorname{GTM}(N)_{l} \rightarrow \operatorname{GTM}\left(N^{\prime}\right)_{l}$.

Proof. One checks that for a general $g \in F_{2}\left(\varphi_{N}\right), \tilde{\partial}_{1}(g)=x_{01}^{b_{N}(g)} \partial_{1}(g), \tilde{\partial}_{2}(g)=x_{12}^{b_{N}(g)} \partial_{2}(g)$, $\tilde{\partial}_{3}(g)=\partial_{2}(g)$, where $\partial_{i}, \tilde{\partial}_{i}$ is shorthand for the morphisms $F_{2}\left(\varphi_{N}, l\right) \rightarrow P_{4}\left(\varphi_{3, N}, l\right),\left(F_{2}\right)_{l} \rightarrow$ $P_{4}\left(\varphi_{3, N}, l\right)$ induced by their homonyms and $b_{N}: F_{2}\left(\varphi_{N}, l\right) \rightarrow \mathbb{Z}_{l}(N)$ is the map from Lemma 6.4 .

Let $(\lambda, \mu, f, g) \in \underline{\operatorname{GTM}}(N)_{l}$. Let us show that $\left(f, \delta_{N N^{\prime}}(g)\right)$ satisfies the mixed pentagon relation. By Lemma $6.4, b_{N}(g)=0$ so the mixed pentagon relation satisfied by $(f, g)$ can be rewritten

$$
\tilde{\partial}_{3}(g) \tilde{\partial}_{1}(g)=\partial_{0}(f) \tilde{\partial}_{2}(g) \partial_{4}(g)
$$

$\begin{array}{ccccccc}K_{n-1, N} & \stackrel{\delta_{N N^{\prime}}}{\rightarrow} & K_{n-1, N^{\prime}} & P_{n} & \stackrel{\partial_{0}}{\rightarrow} & K_{n, N} \\ \text { As in Section 8, one can show that the diagrams } \tilde{\partial}_{1, \ldots, n}, \partial_{n+1 \downarrow} \downarrow & & \downarrow \tilde{\partial}_{1, \ldots, n}, \partial_{n+1} \\ K_{n, N} & \stackrel{\delta_{N N^{\prime}}}{\longrightarrow} & K_{n, N^{\prime}} & & \partial_{0} \searrow & \downarrow \delta_{N N^{\prime}} \\ & & K_{n, N^{\prime}}\end{array}$

commute.

Applying $\delta_{N N^{\prime}}$ to $(52)$, and with $h:=\delta_{N N^{\prime}}(g)$, we get $\tilde{\partial}_{3}(h) \tilde{\partial}_{1}(h)=\partial_{0}(f) \tilde{\partial}_{2}(h) \partial_{4}(h)$ (the $\tilde{\partial}_{i}, \partial_{i}$ are now morphisms $\left(F_{2}\right)_{l} \rightarrow P_{4}\left(\varphi_{3, N^{\prime}}, l\right), F_{2}\left(\varphi_{N^{\prime}}, l\right) \rightarrow P_{4}\left(\varphi_{3, N^{\prime}}, l\right)$. The restriction of $b_{N}$ 


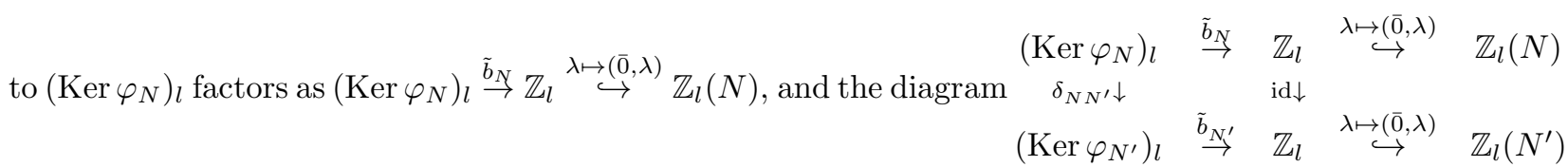

commutes; $b_{N}(g)=0$ then implies that $b_{N^{\prime}}(h)=0$. It follows that $\tilde{\partial}_{i}(h)=\partial_{i}(h)$ for $i=1,2,3$, so $(f, h)$ satisfies the mixed pentagon equation $\partial_{3}(h) \partial_{1}(h)=\partial_{0}(f) \partial_{2}(h) \partial_{4}(h)$.

Let us show that $\left([[\mu]], \delta_{N N^{\prime}}(g)\right)$ satisfies the octogon relations. The inclusion $\left(K_{n, N}\right)_{l} \hookrightarrow$ $P_{n+1}\left(\varphi_{N}, l\right)$ factors through $\left(K_{n, N}\right)_{l} \hookrightarrow K_{n, d}\left(\varphi_{N}, l\right) \hookrightarrow P_{n+1}\left(\varphi_{N}, l\right)$, and we have a commuting

$$
\begin{aligned}
& K_{n, N} \stackrel{\delta_{N N^{\prime}}}{\rightarrow} K_{n, N^{\prime}} \quad\left(K_{n, N}\right)_{l} \stackrel{\delta_{N N^{\prime}}}{\longrightarrow} \quad\left(K_{n, N^{\prime}}\right)_{l} \\
& \text { square } \subset \downarrow \quad \downarrow \downarrow \text {, which gives rise to a commuting square } \quad \subset \downarrow \quad \text {. } \\
& K_{n, d} \stackrel{\delta_{d}}{\rightarrow} \quad P_{n+1} \quad K_{n, d}\left(\varphi_{N}, l\right) \stackrel{\delta_{d}}{\rightarrow} \quad P_{n+1}\left(\varphi_{N^{\prime}}, l\right)
\end{aligned}
$$

For $g \in F_{2}\left(\varphi_{N}, l\right)$, we write $g(x, y)=g, g\left(x^{-1} y^{-1}, y\right)=\kappa(g)$, etc.

Rewriting the octogon identity as

$$
g(x, y)^{-1} y^{\frac{\lambda-1}{2}} g\left(x^{-1} y^{-1}, y\right)\left(x^{-1} y^{-1}\right)^{\mu} x g\left(x^{-2} y^{-1} x, x^{-1} y x\right)^{-1}\left(x^{-1} y x\right)^{\frac{\lambda+1}{2}} g\left(x, x^{-1} y x\right)=x^{1-\mu}
$$

(equality in $\left.F_{2}\left(\varphi_{N}, l\right)\right)$ and multiplying this equation by its conjugates by $x^{-1}, \ldots, x^{1-d}$, we get

$$
\begin{aligned}
& g(x, y)^{-1} y^{\frac{\lambda-1}{2}} g\left(x^{-1} y^{-1}, y\right) \operatorname{Ad}\left[\left(x^{-1} y^{-1}\right)^{\mu} x g\left(x^{-2} y^{-1} x, x^{-1} y x\right)\right]\left[\left(x^{-1} y x\right)^{\lambda}\right] \\
& \operatorname{Ad}\left[\left(x^{-1} y^{-1}\right)^{2 \mu} x^{2} g\left(x^{-3} y^{-1} x^{2}, x^{-2} y x^{2}\right)\right]\left[\left(x^{-2} y x^{2}\right)^{\lambda}\right] \ldots \\
& \operatorname{Ad}\left[\left(x^{-1} y^{-1}\right)^{(d-1) \mu} x^{d-1} g\left(x^{-d} y^{-1} x^{d-1}, x^{1-d} y x^{d-1}\right)\right]\left[\left(x^{1-d} y x^{d-1}\right)^{\lambda}\right] \\
& \left(x^{-1} y^{-1}\right)^{d \mu} g\left(x^{-1} y^{-1}, y\right) y^{\frac{\lambda+1}{2}} g(x, y)=y^{-d \mu} .
\end{aligned}
$$

Now for $t \in\left\{\left(x^{-1} y^{-1}\right)^{d}, x^{d}, x^{\alpha} y x^{-\alpha}\right\}(\alpha \in \mathbb{Z})$, the morphisms $\kappa_{t}: \mathbb{Z} \rightarrow K_{2, d}^{0}, a \mapsto t^{a}$, extend to morphisms $\mathbb{Z}_{l}(N) \stackrel{\kappa_{\left(x^{-1} y-1\right) d}, \kappa_{x^{d}}}{\rightarrow} K_{2, d}^{0}\left(\varphi_{N}, l\right)$ and $\mathbb{Z}_{l} \stackrel{\kappa_{x^{\alpha} x^{-\alpha}}}{\rightarrow} K_{2, d}^{0}\left(\varphi_{N}, l\right)$. For $t \in$ $\left\{x^{-1} y^{-1}, x, y\right\}$, the morphisms $\tilde{\kappa}_{t}: \mathbb{Z} \rightarrow F_{2}, a \mapsto t^{a}$ extend to morphisms $\mathbb{Z}_{l}\left(N^{\prime}\right) \stackrel{\kappa_{x-1}-1, \kappa_{x}}{\rightarrow}$

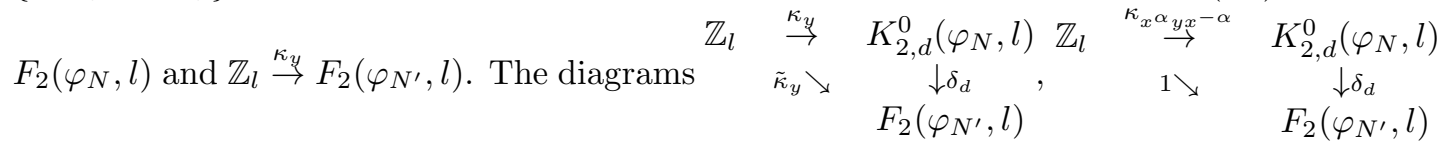

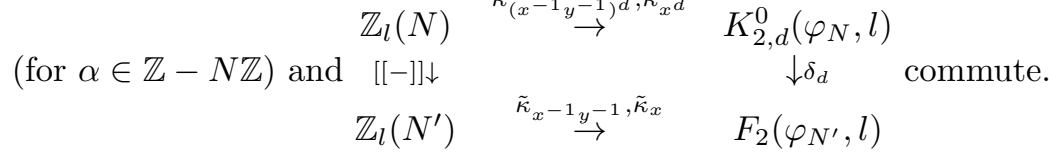

Lemma 9.2. The automorphism $\tilde{\kappa}$ of $F_{2}$ (given by $\kappa(g)=g\left(x^{-1} y^{-1}, y\right)$ ) restricts to an automorphism $\kappa$ of $K_{2, d}^{0} \subset F_{2} ; \tilde{\kappa}$ and $\kappa$ induce automorphisms $\tilde{\kappa} \in \operatorname{Aut}\left(F_{2}\left(\varphi_{N^{\prime}}, l\right)\right)$ and $\kappa \in \operatorname{Aut}\left(K_{2, d}^{0}\left(\varphi_{N}, l\right)\right)$, and the diagram $\begin{array}{ccc}K_{2, d}^{0}\left(\varphi_{N}, l\right) & \stackrel{\kappa}{\delta_{d} \downarrow} & K_{2, d}^{0}\left(\varphi_{N}, l\right) \\ F_{2}\left(\varphi_{N^{\prime}}, l\right) & \stackrel{\tilde{\kappa}}{\rightarrow} F_{2}\left(\varphi_{N^{\prime}}, l\right) & \\ & F_{\delta_{d}} & \end{array}$ commutes.

Proof of Lemma. We have $\varphi_{d} \circ \kappa=-\varphi_{d}$, which implies that $\kappa$ restricts to an automorphism $\tilde{\kappa}$ of $\operatorname{Ker} \varphi_{d}=K_{2, d}^{0}$. Since we also have $\varphi_{N} \circ \kappa=-\varphi_{N}, \varphi_{N^{\prime}} \circ \tilde{\kappa}=-\varphi_{N^{\prime}}, \kappa$ and $\tilde{\kappa}$ also induce automorphisms of the completions $K_{2, d}^{0}\left(\varphi_{N}, l\right)$ and $F_{2}\left(\varphi_{N^{\prime}}, l\right)$. It remains to show that the $K_{2, d}^{0} \stackrel{\kappa}{\rightarrow} K_{2, d}^{0}$

diagram $\delta_{d \downarrow} \downarrow \quad \downarrow \delta_{d}$ commutes. It suffices to check this for the generators $x^{d}, x^{\alpha} y x^{-\alpha}$ $F_{2} \stackrel{\tilde{\kappa}}{\rightarrow} F_{2}$

$(\alpha \in[0, d-1])$ of $K_{2, d}^{0}$. 
We have $\delta_{d}$ ○ $\left(x^{d}\right)=\delta_{d}\left(\left(x^{-1} y^{-1}\right)^{d}\right)=\delta_{d}\left(x^{-d}\left(x^{d-1} y^{-1} x^{1-d}\right) \ldots\left(x y^{-1} x^{-1}\right) y^{-1}\right)=x^{-1} 1 \ldots 1 y^{-1}=$ $x^{-1} y^{-1}=\tilde{\kappa}(x)=\tilde{\kappa} \circ \delta_{d}\left(x^{d}\right) ; \delta_{d} \circ \kappa(y)=\delta_{d}(y)=y=\tilde{\kappa}(y)=\tilde{\kappa} \circ \delta_{d}(y) ;$ and for $\alpha \in[1, d-1]$, $\delta_{d} \circ \kappa\left(x^{\alpha} y x^{-\alpha}\right)=\delta_{d}\left[\left(x^{-1} y^{-1}\right)^{\alpha} y\left(x^{-1} y^{-1}\right)^{-\alpha}\right]=\delta_{d}\left[\operatorname{Ad}\left[y(-1)^{-1} \ldots y(-\alpha)^{-1}\right][y(-\alpha)]\right]=1=$ $\tilde{\kappa}(1)=\tilde{\kappa} \circ \delta_{d}\left(x^{\alpha} y x^{-\alpha}\right)$, where $y(\alpha)=x^{\alpha} y x^{-\alpha}$.

End of proof of Proposition. Identity (53) holds in $K_{2, d}\left(\varphi_{N}, l\right)$ and may be rewritten

$$
\begin{aligned}
& g^{-1} \kappa_{y}\left(\frac{\lambda-1}{2}\right) \kappa(g) \operatorname{Ad}\left[\left(x^{-1} y^{-1}\right)^{\mu} g\left(x^{-1} y^{-1}, y\right)^{-1} x\right]\left[\kappa_{x^{-1} y x}(\lambda)\right] \ldots \operatorname{Ad}\left[\left(x^{-1} y^{-1}\right)^{d \mu} g\left(x^{-1} y^{-1}, y\right)^{-1} x^{d}\right] \\
& {\left[\kappa_{x^{1-d} y x^{d-1}}(\lambda)\right] \kappa_{\left(x^{-1} y^{-1}\right)^{d}}(\mu) \kappa(g) \kappa_{y}\left(\frac{\lambda+1}{2}\right) g=\kappa_{x^{d}}(-\mu) .}
\end{aligned}
$$

Applying $\delta_{d}$ to this identity and using the above commutation relations, we get (with $h:=$ $\left.\delta_{N N^{\prime}}(g)\right)$

$$
h^{-1} \tilde{\kappa}_{y}\left(\frac{\lambda-1}{2}\right) \tilde{\kappa}(h) \tilde{\kappa}_{x^{-1} y^{-1}}([[\mu]]) \tilde{\kappa}(h) \tilde{\kappa}_{y}\left(\frac{\lambda+1}{2}\right) h=\tilde{\kappa}_{x}(-[[\mu]]),
$$

i.e., $([[\mu]], h)$ satisfies the octogon relation.

Let us now show that $\delta_{N N^{\prime}}\left(\underline{g}_{1} \underline{g}_{2}\right)=\delta_{N N^{\prime}}\left(\underline{g}_{1}\right) \delta_{N N^{\prime}}\left(\underline{g}_{2}\right)$ for $\left.\underline{g}_{i}=\left(\lambda_{i}, \mu_{i}, f_{i}, g_{i}\right) \in \underline{\operatorname{GTM}}(N)\right)_{l}$, such that $\overline{\bar{\mu}}_{2} \in(\mathbb{Z} / d \mathbb{Z})^{\times}$(where $\overline{\bar{\mu}}$ is the image of $\bar{\mu} \in \mathbb{Z} / N \mathbb{Z}$ under $\mathbb{Z} / N \mathbb{Z} \rightarrow \mathbb{Z} / d \mathbb{Z}, \overline{1} \mapsto \overline{1}$ ). For this, it suffices to show that $\delta_{N N^{\prime}}\left(\theta_{\left(\lambda_{2}, \mu_{2}, g_{2}\right)}\left(g_{1}\right)\right)=\theta_{\left(\lambda_{2},\left[\left[\mu_{2}\right]\right], \delta_{N N^{\prime}}\left(g_{2}\right)\right)}\left(\delta_{N N^{\prime}}\left(g_{1}\right)\right)$. We will show that we have commutative diagrams

$$
\begin{array}{ccccc}
F_{2}\left(\varphi_{N}, l\right) & \hookleftarrow & K_{2, d}^{0}\left(\varphi_{N}, l\right) & \stackrel{\delta_{d}}{\rightarrow} & F_{2}\left(\varphi_{N^{\prime}}, l\right) \\
\theta_{(\lambda, \mu, g)} \downarrow & & \downarrow & & \downarrow \theta_{\left(\lambda,[[\mu]], \delta_{N N^{\prime}}(g)\right)} \\
F_{2}\left(\varphi_{N}, l\right) & \hookleftarrow & K_{2, d}^{0}\left(\varphi_{N}, l\right) & \stackrel{\delta_{d}}{\rightarrow} & F_{2}\left(\varphi_{N^{\prime}}, l\right)
\end{array}
$$

for any $\lambda \in \mathbb{Z}_{l}, \mu \in \mathbb{Z}_{l}(N)$ such that $\overline{\bar{\mu}} \in(\mathbb{Z} / d \mathbb{Z})^{\times}$and $g \in\left(\operatorname{Ker} \varphi_{N}\right)_{l}$; as $g_{1} \in\left(\operatorname{Ker} \varphi_{N}\right)_{l} \subset$ $K_{2, d}^{0}\left(\varphi_{N}, l\right)$, this implies the wanted equality.

$$
\begin{aligned}
& \text { If } F_{2}\left(\varphi_{N}, l\right) \stackrel{\left(\varphi_{d}\right)_{l}}{\rightarrow} \mathbb{Z} / d \mathbb{Z} \text { is the completion of } \varphi_{d}, \text { we have a commutative diagram } \begin{array}{ccc}
F_{2}\left(\varphi_{N}, l\right) & \stackrel{\left(\varphi_{d}\right)_{l}}{\rightarrow} & \mathbb{Z} / d \mathbb{Z} \\
\theta_{(\lambda, f, g) \downarrow} & \nearrow \bar{\mu}_{\bar{\mu}}\left(\varphi_{d}\right)_{l} & ;
\end{array} \\
& F_{2}\left(\varphi_{N}, l\right)
\end{aligned}
$$

as $K_{2, d}^{0}\left(\varphi_{N}, l\right)=\operatorname{Ker}\left[F_{2}\left(\varphi_{N}, l\right) \stackrel{\left(\varphi_{N}\right)_{l}}{\rightarrow} \mathbb{Z} / d \mathbb{Z}\right]$, this implies that $\theta_{(\lambda, \mu, g)}$ restricts to an automorphism of $K_{2, d}^{0}\left(\varphi_{N}, l\right)$, i.e., the left square of (54) commutes.

Let us now show that the right square commutes. As $K_{2, d}^{0}\left(\varphi_{N}, l\right)$ is topologically generated by $x^{d}$ and the $x^{\alpha} y x^{-\alpha}, \alpha \in[0, d-1]$, it suffices to check this commutativity on these generators. Recall that $\theta_{(\lambda, \mu, g)}$ is defined by $x \mapsto g(x, y) x^{\mu} g(x, y)^{-1}, y \mapsto y^{\lambda}$. Set $h:=$ $\delta_{N N^{\prime}}(g)$. Then $\theta_{(\lambda,[[\mu]], h)} \circ \delta_{d}\left(x^{d}\right)=\theta_{(\lambda,[[\mu]], h)}(x)=h(x, y) x^{[[\mu]]} h(x, y)^{-1}$, while $\delta_{d} \circ \theta_{(\lambda, \mu, g)}\left(x^{d}\right)=$ $\delta_{d}\left(g(x, y) x^{d \mu} g(x, y)^{-1}\right)=\delta_{d}(g(x, y)) \delta_{d}\left(x^{d \mu}\right) \delta_{d}\left(g(x, y)^{-1}\right)$ (as $x^{d \mu}$ and $g(x, y)$ both belong to $\left.K_{2, d}^{0}\left(\varphi_{N}, l\right)\right)$ so $\delta_{d} \circ \theta_{(\lambda, \mu, g)}\left(x^{d}\right)=h(x, y) x^{[[\mu]]} h(x, y)^{-1}=\theta_{(\lambda,[[\mu]], h)} \circ \delta_{d}\left(x^{d}\right)$.

We have $\theta_{(\lambda,[[\mu]], h)} \circ \delta_{d}(y)=\theta_{(\lambda,[[\mu]], h)}(y)=y^{\lambda}$, while $\delta_{d} \circ \theta_{(\lambda, \mu, g)}(y)=\delta_{d}\left(y^{\lambda}\right)=y^{\lambda}$; if now $\alpha \in$ $[1, d-1]$, then $\theta_{(\lambda,[[\mu]], h)} \circ \delta_{d}\left(x^{\alpha} y x^{-\alpha}\right)=1$ while $\delta_{d} \circ \theta_{(\lambda, \mu, g)}\left(x^{\alpha} y x^{-\alpha}\right)=\delta_{d}\left[\operatorname{Ad}\left\{g(x, y) x^{\alpha \mu} g(x, y)^{-1}\right\}\left\{y^{\lambda}\right\}\right]=$ $\delta_{d}\left[\operatorname{Ad}\left\{g(x, y) g\left(x, x^{\alpha \mu} y x^{-\alpha \mu}\right)^{-1}\right\}\left\{\left(x^{\alpha \mu} y x^{-\alpha \mu}\right)^{\lambda}\right\}\right]=\operatorname{Ad}\left\{\delta_{d}\left[g(x, y) g\left(x, x^{\alpha \mu} y x^{-\alpha \mu}\right)^{-1}\right]\right\}\left\{\delta_{d}\left[\left(x^{\alpha \mu} y x^{-\alpha \mu}\right)^{\lambda}\right]\right\}$ as $g(x, y) g\left(x, x^{\alpha \mu} y x^{-\alpha \mu}\right)^{-1} \in K_{2, d}^{0}\left(\varphi_{N}, l\right)$; now $\delta_{d}\left(x^{\alpha \mu} y x^{-\alpha \mu}\right)=1$ as $\bar{\alpha} \overline{\bar{\mu}} \neq 0$ in $\mathbb{Z} / d \mathbb{Z}$, so $\delta_{d} \circ \theta_{(\lambda,[[\mu]], h)}\left(x^{\alpha} y x^{-\alpha}\right)=1=\theta_{(\lambda, \mu, g)} \circ \delta_{d}\left(x^{\alpha} y x^{-\alpha}\right)$. This proves the commutativity of (54), as wanted.

9.3. The subgroup $\operatorname{GTMD}(N)_{l} \subset \operatorname{GTM}(N)_{l}$. Let $\underline{y}:=(1,1,1, y) \in \underline{\operatorname{GTM}}(N)_{l}$; this is the image of $1 \in \mathbb{Z} \subset \mathbb{Z} \rtimes \mathbb{Z} / 2 \mathbb{Z}=\underline{\text { GTM. }}$. The group morphism $\mathbb{Z} \rightarrow F_{2}, s \mapsto y^{s}$ extends to a morphism $\mathbb{Z}_{l} \rightarrow F_{2}\left(\varphi_{N}, l\right)$, denoted again $s \mapsto y^{s} ;$ for $s \in \mathbb{Z}_{l}$, we set $\underline{y}^{s}:=\left(1,1,1, y^{s}\right) \in$ $\underline{\operatorname{GTM}}(N)_{l}$. 
For $\underline{g}=(\lambda, \mu, f, g) \in \underline{\operatorname{GTM}}(N)_{l}$, set $\chi_{l}(\underline{g}):=\lambda \in \mathbb{Z}_{l}$. Then for any $\underline{g} \in \underline{\operatorname{GTM}}(N)_{l}$ and any $s \in \mathbb{Z}_{l}$, we have $\underline{y}^{s} * \underline{g}=\underline{g} * \underline{y}^{\chi_{l}(\underline{g}) s}$, where $*$ is the product on $\underline{\operatorname{GTM}}(N)_{l}$. It follows that any cocycle $\rho: \operatorname{GTM}(N)_{l} \rightarrow \mathbb{Z}_{l}$ (i.e., $\left.\rho\left(\underline{g}_{1} * \underline{g}_{2}\right)=\rho\left(\underline{g}_{1}\right) \chi_{l}\left(\underline{g}_{2}\right)+\rho\left(\underline{g}_{2}\right)\right)$ gives rise to an automorphism $a_{\rho}$ of $\operatorname{GTM}(N)_{l}$, given by $a_{\rho}(\underline{g}):=\underline{g} * \underline{y}^{\rho(\underline{g})}$, i.e., $a_{\rho}(\lambda, \mu, f, g)=\left(\lambda, \mu, f, y^{\rho(\underline{g})} g\right)$.

Lemma 9.3. There is a unique cocycle $c_{N N^{\prime}}: \operatorname{GTM}(N)_{l} \rightarrow \mathbb{Z}_{l}$, defined by $c_{N N^{\prime}}(\lambda, \mu, f, g):=$ $c^{\prime}\left(\delta_{N N^{\prime}}(g)\right)-c^{\prime}\left(\pi_{N N^{\prime}}(g)\right)=c^{\prime}\left(\delta_{N N^{\prime}}(g)\right)-c(g)$, where $c: F_{2}\left(\varphi_{N}, l\right) \rightarrow \mathbb{Z}_{l}$ and $c^{\prime}: F_{2}\left(\varphi_{N^{\prime}}, l\right) \rightarrow$ $\mathbb{Z}_{l}$ are the morphisms induced by $x \mapsto 1, y \mapsto 0$.

Proof. We will prove that each of the maps $(\lambda, \mu, f, g) \mapsto c(g), c^{\prime}\left(\delta_{N N^{\prime}}(g)\right)$ is a cocycle. If $\left(\lambda_{1}, \mu_{1}, f_{1}, g_{1}\right) *\left(\lambda_{2}, \mu_{2}, f_{2}, g_{2}\right)=(\lambda, \mu, f, g)$, then $y^{c(g)}=g(1, y)=g_{1}\left(1, y^{\lambda_{2}}\right) g_{2}(1, y)=$ $y^{\lambda_{2} c\left(g_{1}\right)+c\left(g_{2}\right)}$, so $c(\underline{g})=c\left(\underline{g}_{1}\right) \chi\left(\underline{g}_{2}\right)+c\left(\underline{g}_{2}\right)$.

On the other hand, $g(x, y)=g_{1}\left(g_{2}(x, y) x^{\mu_{2}} g_{2}(x, y)^{-1}, y^{\lambda_{2}}\right) g_{2}(x, y)$; the two factors of this product belong to $\operatorname{Ker}\left(F_{2}\left(\varphi_{N}, l\right) \stackrel{\varphi_{l}}{\rightarrow} \mathbb{Z} / d \mathbb{Z}\right)$, and $c^{\prime} \circ \delta_{N N^{\prime}}: \operatorname{Ker} \varphi_{d} \rightarrow \mathbb{Z}_{l}$ is a group morphism, so $c^{\prime} \circ \delta_{N N^{\prime}}(g)=c^{\prime} \circ \delta_{N N^{\prime}}\left[g_{1}\left(g_{2}(x, y) x^{\mu_{2}} g_{2}(x, y)^{-1}, y^{\lambda_{2}}\right)\right]+c^{\prime} \circ \delta_{N N^{\prime}}\left(g_{2}\right)$, so it remains to show that $c^{\prime} \circ \delta_{N N^{\prime}}\left[g_{1}\left(g_{2}(x, y) x^{\mu_{2}} g_{2}(x, y)^{-1}, y^{\lambda_{2}}\right)\right]=\lambda_{2}\left(c^{\prime} \circ \delta_{N N^{\prime}}\right)\left(g_{1}\right)$. If $\tilde{g}_{1} \in\left(F_{d+1}\right) l$ is defined by $g_{1}(x, y)=\tilde{g}_{1}\left(x^{d} \mid y, x y x^{-1}, \ldots, x^{d-1} y x^{1-d}\right)$, then $\tilde{g}_{1}(1 \mid y, 1, \ldots, 1)=y^{c^{\prime} \circ \delta_{N N^{\prime}}\left(g_{1}\right)}$. On the other hand,

$g_{1}\left(g_{2}(x, y) x^{\mu_{2}} g_{2}(x, y)^{-1}\right)=\tilde{g}_{1}\left(g_{2}(x, y) x^{d \mu_{2}} g_{2}(x, y)^{-1} \mid y^{\lambda_{2}}, \operatorname{Ad}\left[g_{2}(x, y) x^{\mu_{2}} g_{2}(x, y)^{-1} x^{-\mu_{2}}\right]\left[x^{\mu_{2}} y^{\lambda_{2}} x^{-\mu_{2}}\right]\right.$,

$\left.\operatorname{Ad}\left[g_{2}(x, y) x^{2 \mu_{2}} g_{2}(x, y)^{-1} x^{-2 \mu_{2}}\right]\left[x^{2 \mu_{2}} y^{\lambda_{2}} x^{-2 \mu_{2}}\right], \ldots\right) \stackrel{\delta_{N N^{\prime}}}{\rightrightarrows} \tilde{g}_{1}\left(1 \mid y^{\lambda_{2}}, 1, \ldots, 1\right)=y^{\lambda_{2}\left(c^{\prime} \circ \delta_{N N^{\prime}}\right)\left(g_{1}\right)}$,

as $x^{d} \stackrel{\delta_{N N^{\prime}}}{\mapsto} 1, y \stackrel{\delta_{N N^{\prime}}}{\mapsto} y$ and $x^{\alpha} y x^{-\alpha} \stackrel{\delta_{N N^{\prime}}}{\mapsto} 1$ for $\alpha \in[1, d-1]$ as $\bar{\mu}_{2} \in(\mathbb{Z} / d \mathbb{Z})^{\times}$. So $c^{\prime}$ 。 $\delta_{N N^{\prime}}\left[g_{1}\left(g_{2}(x, y) x^{\mu_{2}} g_{2}(x, y)^{-1}, y^{\lambda_{2}}\right)\right]=\lambda_{2}\left(c^{\prime} \circ \delta_{N N^{\prime}}\right)\left(g_{1}\right)$, as wanted.

We denote the resulting automorphism of $\operatorname{GTM}(N)_{l}$ by $a_{N N^{\prime}}$, Since $\pi_{N N^{\prime}}, \delta_{N N^{\prime}}: \operatorname{GTM}(N)_{l} \rightarrow$ $\operatorname{GTM}\left(N^{\prime}\right)_{l}$ are group morphisms,

$$
\begin{aligned}
& \operatorname{GTMD}(N)_{l}:=\left\{\underline{g} \in \operatorname{GTM}(N)_{l}\left|\forall N^{\prime}\right| N, \delta_{N N^{\prime}}(\underline{g})=\pi_{N N^{\prime}} \circ a_{N N^{\prime}}(\underline{g})\right\} \\
& =\left\{(\lambda, \mu, f, g) \in \operatorname{GTM}(N)_{l}\left|\forall N^{\prime}\right| N, \delta_{N N^{\prime}}(g)=y^{c^{\prime}\left(\delta_{N N^{\prime}}(g)\right)-c(g)} \pi_{N N^{\prime}}(g)\right\}
\end{aligned}
$$

is a subgroup of $\operatorname{GTM}(N)_{l}$.

Proposition 9.4. The morphism $\widehat{\operatorname{GTM}} \rightarrow \operatorname{GTM}(N)_{l}$ factors through $\widehat{\operatorname{GTM}} \rightarrow \operatorname{GTMD}(N)_{l} \hookrightarrow$ $\operatorname{GTM}(N)_{l}$.

Proof. Recall the cocycle $c_{N}$ of $\widehat{\mathrm{GTM}}$ constructed in Section 9.1, then the diagram

$\begin{array}{cccc}\widehat{\mathrm{GTM}} & \stackrel{c_{d}}{\longrightarrow} & \widehat{\mathbb{Z}} \\ \downarrow & & \downarrow \\ \operatorname{GTM}(N)_{l} & \stackrel{c_{N N^{\prime}}}{\longrightarrow} & \mathbb{Z}_{l}\end{array}$

commutes. Recall that $c_{d}$ gives rise to an automorphism $\hat{a}_{d} \in \operatorname{Aut}(\widehat{\mathrm{GTM}}),(\lambda, \mu, f, g) \mapsto$ $\left(\lambda, \mu, f, y^{\hat{c}_{d}(f)} g\right)$. We have commutative diagrams

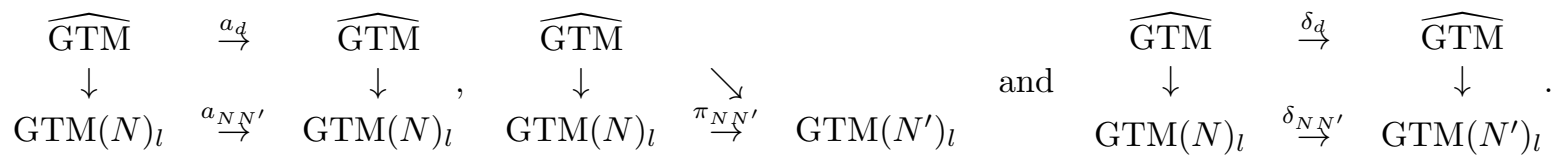

We have seen (Theorem 8.1) that $\delta_{d}=a_{d} \in \operatorname{Aut}(\widehat{\mathrm{GTM}})$; it follows that for any $\underline{g} \in \operatorname{Im}(\widehat{\mathrm{GTM}} \rightarrow$ $\left.\operatorname{GTM}(N)_{l}\right)$, we have $\delta_{N N^{\prime}}(\underline{g})=\pi_{N N^{\prime}} \circ a_{N N^{\prime}}(\underline{g})$.

Let $\operatorname{Div}(N)$ be the partial semigroup (for the multiplication) of all the divisors of $N$. 
Proposition 9.5. 1) We have a group morphism $\operatorname{GTMD}(N)_{l} \rightarrow \operatorname{Hom}\left(\operatorname{Div}(N), \mathbb{Z}_{l}\right) \simeq \mathbb{Z}_{l}^{\nu}$ (here $\nu$ is the number of distinct prime powers in the decomposition of $N)$, taking $(\lambda, \mu, f, g)$ to $\left[d \mapsto c_{N N^{\prime}}(g)\right]$.

2) The group morphisms $\delta_{N N^{\prime}}, \pi_{N N^{\prime}}: \operatorname{GTM}(N)_{l} \rightarrow \operatorname{GTM}\left(N^{\prime}\right)_{l}$ restrict to morphisms $\operatorname{GTMD}(N)_{l} \rightarrow \operatorname{GTMD}\left(N^{\prime}\right)_{l}$.

Proof. Both statements are consequences of the following equalities: if $N^{\prime \prime}\left|N^{\prime}\right| N$, then $\delta_{N^{\prime} N^{\prime \prime}} \circ \delta_{N N^{\prime}}=\delta_{N N^{\prime \prime}}, \pi_{N^{\prime} N^{\prime \prime}} \circ \pi_{N N^{\prime}}=\pi_{N N^{\prime \prime}}$, and if $d_{1}, d_{2}, d_{1} d_{2} \in \operatorname{Div}(N), N_{i}=N / d_{i}$ $(i=1,2), N_{3}=N /\left(d_{1} d_{2}\right)$, then $\pi_{N_{1} N_{3}} \circ \delta_{N N_{1}}=\delta_{N_{2} N_{3}} \circ \pi_{N N_{2}}$.

9.4. The subgroup $\operatorname{GTMD}(N, \mathbf{k}) \subset \operatorname{GTM}(N, \mathbf{k})$. One defines as above morphisms $\pi_{N N^{\prime}}, \delta_{N N^{\prime}}$ : $\operatorname{GTM}(N, \mathbf{k}) \rightarrow \operatorname{GTM}\left(N^{\prime}, \mathbf{k}\right)$, a cocycle $c_{N N^{\prime}}: \operatorname{GTM}(N, \mathbf{k}) \rightarrow \mathbf{k}$ and the $\operatorname{subgroup} \operatorname{GTMD}(N, \mathbf{k}) \subset$ $\operatorname{GTM}(N, \mathbf{k})$. We have as above a group morphism $\operatorname{GTMD}(N, \mathbf{k}) \rightarrow \operatorname{Hom}(\operatorname{Div}(N), \mathbf{k}) \simeq \mathbf{k}^{\nu}$. When $\mathbf{k}=\mathbb{Q}_{l}$, these morphisms are compatible with the morphisms $\operatorname{GTM}(N)_{l} \hookrightarrow \operatorname{GTM}\left(N, \mathbb{Q}_{l}\right)$, in particular $\operatorname{GTMD}(N)_{l} \hookrightarrow \operatorname{GTMD}\left(N, \mathbb{Q}_{l}\right)$.

\section{Distribution relations and the torsor $\operatorname{Psdist}(N, \mathbf{k})$}

Recall that $N, N^{\prime}$ are integers $\geq 1$ with $N^{\prime} \mid N$; we set $d:=N / N^{\prime}$.

10.1. The morphisms $\pi_{N N^{\prime}}: \underline{\operatorname{Pseudo}}(N, \mathbf{k}) \rightarrow \underline{\operatorname{Pseudo}}\left(N^{\prime}, \mathbf{k}\right), \operatorname{GRTM}(N, \mathbf{k}) \rightarrow \operatorname{GRTM}\left(N^{\prime}, \mathbf{k}\right)$.

10.1.1. The inclusion $K_{n, N} \stackrel{\pi_{N N^{\prime}}}{\longrightarrow} K_{n, N^{\prime}}\left(\subset P_{n+1}\right)$ may be viewed as associated to $W_{n, N} \rightarrow$ $W_{n, N^{\prime}},\left(w_{1}, \ldots, w_{n}\right) \mapsto\left(w_{1}^{d}, \ldots, w_{n}^{d}\right)$. This group morphism induces a Lie algebra morphism Lie $K_{n, N} \rightarrow$ Lie $K_{n, N^{\prime}}$, given by $\Xi_{0 i} \mapsto d \Xi_{0 i}^{\prime}, \xi_{i j}(\alpha) \mapsto\left(q \Xi_{0 i}^{\prime}\right) * \xi_{i j}^{\prime}\left(\alpha^{\prime}\right) *\left(-q \Xi_{0 i}^{\prime}\right)$ for $\alpha=N^{\prime} q+\alpha^{\prime}$, $\alpha^{\prime} \in\left[0, N^{\prime}-1\right]$ (the primes denote the generators of Lie $K_{n, N^{\prime}}$ ). This morphism is not injective, e.g., $\xi_{i j}\left(\alpha+N^{\prime}\right)$ and $\left(\Xi_{0 i} / d\right) * \xi_{i j}(\alpha) *\left(-\Xi_{0 i} / d\right)$ have the same image.

The associated graded Lie algebra morphism is $\pi_{N N^{\prime}}: \mathfrak{t}_{n, N} \rightarrow \mathfrak{t}_{n, N^{\prime}}$ given by

$$
t_{0}^{0 i} \mapsto d t_{0}^{\prime 0 i}, t^{i j}(a) \mapsto t^{\prime i j}(\bar{a}) ;
$$

here $t_{0}^{\prime 0 i}, t^{\prime i j}(a)$ are the generators of $\mathfrak{t}_{n, N^{\prime}}$ and $\bar{a}$ is the image of $a$ under $\mathbb{Z} / N \mathbb{Z} \rightarrow \mathbb{Z} / N^{\prime} \mathbb{Z}$, $\overline{1} \mapsto \overline{1}$.

10.1.2. The morphisms $\pi_{N N^{\prime}}: \operatorname{GRTM}(N, \mathbf{k}) \rightarrow \operatorname{GRTM}\left(N^{\prime}, \mathbf{k}\right)$. The morphisms $\pi_{N N^{\prime}}: \mathfrak{t}_{n, N} \rightarrow$ $\mathfrak{t}_{n, N^{\prime}}$ are compatible with the insertion-coproduct maps. Recall that $\pi_{N N^{\prime}}: \mathfrak{t}_{3, N}^{0} \rightarrow \mathfrak{t}_{3, N^{\prime}}^{0}$ is defined by

$$
A \mapsto d A^{\prime}, b(a) \mapsto b^{\prime}(\bar{a}) .
$$

Then $\pi_{N N^{\prime}}$ induces a group morphism $\operatorname{GRTM}_{(\overline{1}, 1)}(N, \mathbf{k}) \rightarrow \operatorname{GRTM}_{(\overline{1}, 1)}\left(N^{\prime}, \mathbf{k}\right)$, by $(h, k) \mapsto$ $\left(h, \pi_{N N^{\prime}}(k)\right)$; together with the morphism $(\mathbb{Z} / N \mathbb{Z})^{\times} \times \mathbf{k}^{\times} \stackrel{\operatorname{can} \times \text { id }}{\rightarrow}\left(\mathbb{Z} / N^{\prime} \mathbb{Z}\right)^{\times} \times \mathbf{k}^{\times}$, this extends to a morphism $\operatorname{GRTM}(N, \mathbf{k}) \rightarrow \operatorname{GRTM}\left(N^{\prime}, \mathbf{k}\right)$. In the same way, $\pi_{N N^{\prime}}$ induces a Lie algebra morphism $\operatorname{grtm}_{(\overline{1}, 1)}(N, \mathbf{k}) \rightarrow \mathfrak{g r t m}_{(\overline{1}, 1)}\left(N^{\prime}, \mathbf{k}\right)$, graded and compatible with the actions of $(\mathbb{Z} / N \mathbb{Z})^{\times}$and $\left(\mathbb{Z} / N^{\prime} \mathbb{Z}\right)^{\times}$. It extends to a morphism $\mathfrak{g r t m}_{\overline{1}}(N, \mathbf{k}) \rightarrow \mathfrak{g r t m}_{\overline{1}}\left(N^{\prime}, \mathbf{k}\right)$, commuting with the projections $\mathfrak{g r t m}_{\overline{1}}\left(N^{\prime \prime}, \mathbf{k}\right) \rightarrow \mathbf{k}, N^{\prime \prime}=N, N^{\prime}$.

10.1.3. The morphisms $\pi_{N N^{\prime}}: \underline{\operatorname{Pseudo}}(N, \mathbf{k}) \rightarrow \underline{\operatorname{Pseudo}}\left(N^{\prime}, \mathbf{k}\right)$. There is a unique morphism $\underline{\operatorname{Pseudo}}(N, \mathbf{k}) \rightarrow \underline{\operatorname{Pseudo}}\left(N^{\prime}, \mathbf{k}\right)$, taking $(a, \lambda, \Phi, \Psi)$ to $\left(\bar{a}, \lambda, \Phi, \pi_{N_{N^{\prime}}}(\Psi)\right)$. This morphism is compatible with the morphism $\operatorname{GRTM}(N, \mathbf{k}) \stackrel{\pi_{N N^{\prime}}}{\longrightarrow} \operatorname{GRTM}\left(N^{\prime}, \mathbf{k}\right)$, and it restricts to a morphism of torsors $\operatorname{Pseudo}(N, \mathbf{k}) \rightarrow \operatorname{Pseudo}\left(N^{\prime}, \mathbf{k}\right)$.

10.2. The morphisms $\delta_{N N^{\prime}}: \operatorname{Pseudo}(N, \mathbf{k}) \rightarrow \operatorname{Pseudo}\left(N^{\prime}, \mathbf{k}\right), \operatorname{GrTM}(N, \mathbf{k}) \rightarrow \operatorname{GrTM}\left(N^{\prime}, \mathbf{k}\right)$. If $a \in \mathbb{Z} / N \mathbb{Z}$, we write $d \mid a$ iff $a \in d \mathbb{Z} / N \mathbb{Z}$. We then denote by $a / d$ the unique element $a^{\prime} \in \mathbb{Z} / N^{\prime} \mathbb{Z}$ such that $d a^{\prime}=a$. 
10.2.1. Recall that the morphism $\delta_{N N^{\prime}}: K_{n, N} \rightarrow K_{n, N^{\prime}}$ may be viewed as induced by the morphism $W_{n, N} \rightarrow W_{n, N^{\prime}},\left(w_{1}, \ldots, w_{n}\right) \mapsto\left(w_{1}, \ldots, w_{n}\right)$. It is given by $X_{0 i} \mapsto X_{0 i}^{\prime}, x_{i j}(\alpha) \mapsto$ $x_{i j}^{\prime}(\alpha / d)$ if $d \mid \alpha, x_{i j}(\alpha) \mapsto 1$ if $d \nmid \alpha$.

The associated graded morphism of Lie $\delta_{N N^{\prime}}$ is $\delta_{N N^{\prime}}: \mathfrak{t}_{n, N} \rightarrow \mathfrak{t}_{n, N^{\prime}}$, given by

$$
t_{0}^{0 i} \mapsto t_{0}^{\prime 0 i}, \quad t^{i j}(a) \mapsto t^{\prime i j}(a / d) \text { if } d \mid a, t^{i j}(a) \mapsto 0 \text { if } d \nmid a .
$$

10.2.2. The morphisms $\delta_{N N^{\prime}}: \operatorname{GRTM}(N, \mathbf{k}) \rightarrow \operatorname{GRTM}\left(N^{\prime}, \mathbf{k}\right)$. The morphisms $\delta_{N N^{\prime}}: \mathfrak{t}_{n, N} \rightarrow$ $\mathfrak{t}_{n, N^{\prime}}$ are compatible with the insertion-coproduct morphisms. Recall that $\delta_{N N^{\prime}}: \mathfrak{t}_{3, N}^{0} \rightarrow \mathfrak{t}_{3, N^{\prime}}^{0}$ is defined by

$$
A \mapsto A^{\prime}, \quad b(a) \mapsto b^{\prime}(a / d) \text { if } d \mid a, b(a) \mapsto 0 \text { if } d \nmid a .
$$

One checks that there is a unique group morphism $\delta_{N N^{\prime}}: \operatorname{GRTM}_{(\overline{1}, 1)}(N, \mathbf{k}) \rightarrow \operatorname{GRTM}_{(\overline{1}, 1)}\left(N^{\prime}, \mathbf{k}\right)$, taking $(h, k)$ to $\left(h, \delta_{N N^{\prime}}(k)\right)$. In the same way as above, its extends to a group morphism $\delta_{N N^{\prime}}: \operatorname{GRTM}(N, \mathbf{k}) \rightarrow \operatorname{GRTM}\left(N^{\prime}, \mathbf{k}\right)$. The Lie algebra versions of these morphisms are $\delta_{N N^{\prime}}: \mathfrak{g r t m}_{\overline{1}}(N, \mathbf{k}) \rightarrow \mathfrak{g r t m}_{\overline{1}}\left(N^{\prime}, \mathbf{k}\right)$ and $\delta_{N N^{\prime}}: \mathfrak{g r t m}_{(\overline{1}, 1)}(N, \mathbf{k}) \rightarrow \mathfrak{g r t m}_{(\overline{1}, 1)}\left(N^{\prime}, \mathbf{k}\right)$ with the same properties as above.

10.2.3. The morphisms $\delta_{N N^{\prime}}: \operatorname{Pseudo}(N, \mathbf{k}) \rightarrow \operatorname{Pseudo}\left(N^{\prime}, \mathbf{k}\right)$. There is a unique morphism $\underline{\text { Pseudo }}(N, \mathbf{k}) \rightarrow \underline{\text { Pseudo }}(N, \mathbf{k})$ taking $(a, \lambda, \Phi, \Psi)$ to $\left(\bar{a}, \lambda, \Phi, \delta_{N N^{\prime}}(\Psi)\right)$, and it shares the properties of $\pi_{N N^{\prime}}: \underline{\text { Pseudo }}(N, \mathbf{k}) \rightarrow \underline{\text { Pseudo }}(N, \mathbf{k})$ stated above.

10.3. The case $N^{\prime}=1$.

Lemma 10.1. The compositions of $\mathfrak{g r t m}_{(\overline{1}, 1)}(N, \mathbf{k}) \stackrel{\pi_{N 1}}{\rightarrow} \mathfrak{g r t m}_{(\overline{1}, 1)}(1, \mathbf{k}) \simeq \mathfrak{g r t}_{1}(\mathbf{k}) \oplus \mathbf{k}\left(0, t^{23}\right)$ and $\mathfrak{g r t m}_{(\overline{1}, 1)}(N, \mathbf{k}) \stackrel{\delta_{N 1}}{\rightarrow} \mathfrak{g r t m}_{(\overline{1}, 1)}(1, \mathbf{k}) \simeq \mathfrak{g r t}_{1}(\mathbf{k}) \oplus \mathbf{k}\left(0, t^{23}\right)$ with projection on the first component both coincide with the canonical morphism $\mathfrak{g r t m}_{(\overline{1}, 1)}(N, \mathbf{k}) \rightarrow \mathfrak{g r t}_{1}(\mathbf{k})$.

Proof. Denote by $\psi \mapsto \bar{\psi}$ the morphism $\pi_{N 1}: \mathfrak{t}_{3, N}^{0} \rightarrow \mathfrak{t}_{3}^{0}$ defined by $A \mapsto N t^{12}, b(a) \mapsto t^{23}$ and $\varepsilon_{0}: \mathfrak{t}_{n, N} \rightarrow \mathfrak{t}_{n}$ the morphism $t_{0}^{0 i} \mapsto 0, t(a)^{i j}=\delta_{a, \overline{0}} t^{i j}$. Then $\varepsilon_{0}(\bar{\psi})$ is proportional to $t^{12}$, so it is zero if $\psi$ has degree $>1$. On the other hand, (40) implies that $\varphi^{1,2,3}+\bar{\psi}^{0,12,3}+\bar{\psi}^{0,1,2}=$ $\bar{\psi}^{01,2,3}+\bar{\psi}^{0,1,23}$. Applying $\varepsilon_{0}$ to this identity, we get $\bar{\psi}=\varphi$. The case of $\delta_{N 1}$ is similar.

$\pi_{N 1}$ is related to the canonical projection $\underline{\operatorname{Pseudo}}(N, \mathbf{k}) \rightarrow \underline{\operatorname{Assoc}}(\mathbf{k}), \Psi \mapsto \Phi$ as follows:

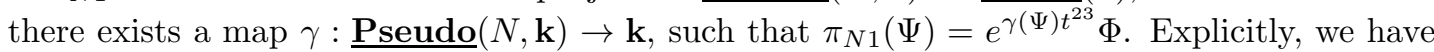
$\gamma(\Psi)=N \gamma_{A}(\Psi)+\sum_{a \in \mathbb{Z} / N \mathbb{Z}} \gamma_{a}(\Psi)$, where $\gamma_{A}(\Psi), \gamma_{a}(\Psi)$ are the coefficients of $A, b(a)$ in $\Psi$.

In the same way, if we set $\gamma^{\prime}(\Psi):=\gamma_{A}(\Psi)+\gamma_{0}(\Psi)$, then $\delta_{N 1}(\Psi)=e^{\gamma^{\prime}(\Psi) t^{23}} \Phi$.

\subsection{The scheme $\underline{\operatorname{Psdist}}(N, \mathbf{k})$ and the groups $\operatorname{GTMD}(N, \mathbf{k})$ and $\operatorname{GRTMD}(N, \mathbf{k})$.}

10.4.1. The scheme $\underline{\operatorname{Psdist}}(N, \mathbf{k})$. Define $\underline{\operatorname{Psdist}}(N, \mathbf{k}) \subset \underline{\operatorname{Pseudo}}(N, \mathbf{k})$ as the subset of all $(a, \lambda, \Phi, \Psi)$ such that

$$
\forall N^{\prime} \mid N, \pi_{N N^{\prime}}(\Psi)=e^{\rho_{N N^{\prime}}(\Psi) b(0)} \delta_{N N^{\prime}}(\Psi) .
$$

Here $\rho_{N N^{\prime}}(\Psi)=\rho\left(\pi_{N N^{\prime}}(\Psi)\right)-\rho(\Psi)$, where $\rho(\tilde{\Psi})$ is the coefficient of $b(0)$ in $\tilde{\Psi} \in \exp \left(\hat{\mathfrak{t}}_{2, N^{\prime \prime}}^{0}\right)$ $\left(N^{\prime \prime}=N, N^{\prime}\right)$.

Lemma 10.2. For any $(a, \lambda, \Psi, \Psi) \in \underline{\operatorname{Psdist}}(N, \mathbf{k})$, the map $\operatorname{Div}(N) \ni d \mapsto \rho_{N, N / d}(\Psi)$ is in $\operatorname{Hom}(\operatorname{Div}(N), \mathbf{k})$; this defines a map $\underline{\operatorname{Psdist}}(N, \mathbf{k}) \rightarrow \operatorname{Hom}(\operatorname{Div}(N), \mathbf{k})$.

Proof. This again follows from the identities $\pi_{N^{\prime} N^{\prime \prime}} \circ \pi_{N N^{\prime}}=\pi_{N N^{\prime \prime}}, \delta_{N^{\prime} N^{\prime \prime}} \circ \delta_{N N^{\prime}}=\delta_{N N^{\prime \prime}}$ and if $d_{1}, d_{2}, d_{3}:=d_{1} d_{2} \in \operatorname{Div}(N), N_{i}=N / d_{i}$, then $\pi_{N_{1} N_{3}} \circ \delta_{N N_{1}}=\delta_{N_{2} N_{3}} \circ \pi_{N N_{2}}$ (morphisms between Lie algebras $\hat{\mathfrak{t}}_{2, \nu}^{0}$ instead of groups $\left.\left(K_{2, \nu}\right)_{l}\right)$.

We will define $\operatorname{Psdist}(N, \mathbf{k}):=\underline{\operatorname{Psdist}}(N, \mathbf{k}) \cap \operatorname{Pseudo}(N, \mathbf{k})$, so this is the subset of $\underline{\operatorname{Psdist}}(N, \mathbf{k})$ defined by $(a, \lambda) \in(\mathbb{Z} / N \mathbb{Z})^{\times} \times \mathbf{k}^{\times}$. 
10.4.2. The group $\operatorname{GRTMD}(N, \mathbf{k})$. We have morphisms $\pi_{N N^{\prime}}, \delta_{N N^{\prime}}: \operatorname{GRTM}_{(\overline{1}, 1)}(N, \mathbf{k}) \rightarrow$ $\operatorname{GRTM}_{(\overline{1}, 1)}\left(N^{\prime}, \mathbf{k}\right)$, given by $(h, k) \mapsto\left(h, \pi_{N N^{\prime}}(k)\right)$ and $(h, k) \mapsto\left(h, \delta_{N N^{\prime}}(k)\right)$. We also have $a_{N N^{\prime}} \in \operatorname{Aut}\left(\operatorname{GRTM}_{(\overline{1}, 1)}(N, \mathbf{k})\right)$, defined by $a_{N N^{\prime}}(h, k):=\left(h, e^{\rho_{N N^{\prime}}(k) b(0)} k\right)$.

We then set

$$
\begin{aligned}
\operatorname{GRTMD}_{(\overline{1}, 1)}(N, \mathbf{k}): & =\left\{(h, k) \in \operatorname{GRTM}_{(\overline{1}, 1)}(N, \mathbf{k})\left|\forall N^{\prime}\right| N, \pi_{N N^{\prime}}(h, k)=\delta_{N N^{\prime}} \circ a_{N N^{\prime}}(h, k)\right\} \\
& =\left\{(h, k) \in \operatorname{GRTM}_{(\overline{1}, 1)}(N, \mathbf{k}) \mid \pi_{N N^{\prime}}(k)=e^{\rho_{N N^{\prime}}(k) b(0)} \delta_{N N^{\prime}}(k)\right\} .
\end{aligned}
$$

Then $\operatorname{GRTMD}_{(\overline{1}, 1)}(N, \mathbf{k})$ is a subgroup of $\operatorname{GRTM}_{(\overline{1}, 1)}(N, \mathbf{k})$, which is preserved by the action of $(\mathbb{Z} / N \mathbb{Z})^{\times} \times \mathbf{k}^{\times}$. Set $\operatorname{GRTMD}(N, \mathbf{k}):=\operatorname{GRTMD}_{(\overline{1}, 1)}(N, \mathbf{k}) \rtimes\left((\mathbb{Z} / N \mathbb{Z})^{\times} \times \mathbf{k}^{\times}\right)$.

Lemma 10.3. If $(h, k) \in \operatorname{GRTMD}_{(\overline{1}, 1)}(N, \mathbf{k})$, then $\operatorname{Div}(N) \ni d \mapsto \rho_{N, N / d}(k)$ belongs to $\operatorname{Hom}(\operatorname{Div}(N), \mathbf{k}) \simeq \mathbf{k}^{\nu}$. This defines a group morphism $\operatorname{GRTMD}_{(\overline{1}, 1)}(N, \mathbf{k}) \rightarrow \mathbf{k}^{\nu}$.

Proof. The proof is the same as above; the group morphism property is checked directly.

This group morphism is compatible with the projection $(\mathbb{Z} / N \mathbb{Z})^{\times} \times \mathbf{k}^{\times} \rightarrow \mathbf{k}^{\times}$and the scaling action of $\mathbf{k}^{\times}$on $\mathbf{k}^{\nu}$. We thus get a group morphism $\operatorname{GRTMD}(N, \mathbf{k}) \rightarrow(\mathbb{Z} / N \mathbb{Z})^{\times} \times\left(\mathbf{k}^{\nu} \rtimes \mathbf{k}^{\times}\right)$.

Denote by $\mathfrak{g r t m} \mathfrak{d}_{\overline{1}, 1)}(N, \mathbf{k}) \subset \mathfrak{g r t m}_{\overline{1}}(N, \mathbf{k})$ the Lie algebras of $\operatorname{GRTMD}_{(\overline{1}, 1)}(N, \mathbf{k}) \subset \operatorname{GRTMD}(N, \mathbf{k})$. Let $\mathfrak{g r t m}_{\overline{1}}(N, \mathbf{k})=\mathbf{k} \oplus \mathfrak{g r t m} \mathfrak{( 1}_{(\overline{1}, 1)}(N, \mathbf{k})_{1} \oplus \mathfrak{g r t m} \mathfrak{m}_{(\overline{1}, 1)}(N, \mathbf{k})_{2} \oplus \cdots$ be the decomposition of $\mathfrak{g r t m}_{\overline{1}}(N, \mathbf{k})$ in homogeneous components. Then the decomposition of $\mathfrak{g r t m}_{\overline{1}}(N, \mathbf{k})$ is $\mathbf{k} \oplus \mathfrak{g r t m} \mathfrak{d}_{(\overline{1}, 1)}(N, \mathbf{k})_{1} \oplus$ $\mathfrak{g r t m o}_{(\overline{1}, 1)}(N, \mathbf{k})_{2} \oplus \cdots$, where

$$
\mathfrak{g r t m}_{(\overline{1}, 1)}(N, \mathbf{k})_{1}=\left\{x \in \mathfrak{g r t m}_{(\overline{1}, 1)}(N, \mathbf{k})_{1}\left|\forall N^{\prime}\right| N,\left(\pi_{N N^{\prime}}-\delta_{N N^{\prime}}\right)(x)=\rho_{N N^{\prime}}(x) b(0)\right\}
$$

and for $n>1$,

$$
\mathfrak{g r t m}_{(\overline{1}, 1)}(N, \mathbf{k})_{n}=\left\{x \in \mathfrak{g r t m}_{(\overline{1}, 1)}(N, \mathbf{k})_{n}\left|\forall N^{\prime}\right| N, \pi_{N N^{\prime}}(x)=\delta_{N N^{\prime}}(x)\right\} .
$$

The element $1 \in \mathbf{k}$ is such that $[1, \psi]=n \psi$ for $\psi$ of degree $n$, and $\mathfrak{g r t m}_{(\overline{1}, 1)}(N, \mathbf{k})$ is the sum of homogeneous components of $\mathfrak{g r t m}_{\overline{1}}(N, \mathbf{k})$ of degree $>0$. The action of $(\mathbb{Z} / N \mathbb{Z})^{\times}$on $\mathfrak{g r t m}_{(\overline{1}, 1)}(N, \mathbf{k})$ restricts to a degree-preserving action on $\mathfrak{g r t m}_{(\overline{1}, 1)}(N, \mathbf{k})$. The Lie algebra morphism $\mathfrak{g r t m} \mathfrak{d}_{(\overline{1}, 1)}(N, \mathbf{k}) \rightarrow \mathbf{k}^{\nu}$ is the direct sum of $\mathfrak{g r t m}_{(\overline{1}, 1)}(N, \mathbf{k})_{1} \rightarrow \mathbf{k}^{\nu}, x \mapsto[d \mapsto$ $\left.\rho_{N, N / d}(x)\right]$, and zero on the other components; this morphism is invariant under the action of $(\mathbb{Z} / N \mathbb{Z})^{\times}$.

10.4.3. Torsor structure of $\boldsymbol{P} \operatorname{sdist}(N, \mathbf{k})$. We have commutative diagrams

$$
\begin{array}{ccc}
\operatorname{GTM}(N, \mathbf{k}) \times \underline{\operatorname{Pseudo}}(N, \mathbf{k}) \times \operatorname{GRTM}(N, \mathbf{k}) & \rightarrow \underline{\operatorname{Pseudo}}(N, \mathbf{k}) \\
\left(\begin{array}{c}
\pi_{N N^{\prime}} \times \pi_{N N^{\prime}} \times \pi_{N N^{\prime}} \\
\left(\text { resp. }, \delta_{N N^{\prime}} \times \delta_{N N^{\prime}} \times \delta_{N N^{\prime}}\right)
\end{array}\right. & \downarrow & \downarrow \pi_{N N^{\prime}}\left(\text { resp., } \delta_{N N^{\prime}}\right) \\
\operatorname{GTM}\left(N^{\prime}, \mathbf{k}\right) \times \underline{\text { Pseudo }}\left(N^{\prime}, \mathbf{k}\right) \times \operatorname{GRTM}\left(N^{\prime}, \mathbf{k}\right) & \rightarrow & \underline{\text { Pseudo }}\left(N^{\prime}, \mathbf{k}\right)
\end{array}
$$

and

$$
\begin{aligned}
& \operatorname{GTM}(N, \mathbf{k}) \times \underline{\text { Pseudo }}(N, \mathbf{k}) \times \operatorname{GRTM}(N, \mathbf{k}) \rightarrow \underline{\text { Pseudo }}(N, \mathbf{k}) \\
& a_{N N^{\prime}} \downarrow \\
& \operatorname{GTM}(N, \mathbf{k}) \times \underline{\operatorname{Pseudo}}(N, \mathbf{k}) \times \operatorname{GRTM}(N, \mathbf{k}) \rightarrow \underline{\operatorname{Pseudo}}(N, \mathbf{k})
\end{aligned}
$$

which implies that the action of $\operatorname{GRTM}(N, \mathbf{k})$ on $\underline{\mathbf{P s e u d o}}(N, \mathbf{k})$ restricts to an action of $\operatorname{GRTMD}(N, \mathbf{k})$ on $\underline{\operatorname{Psdist}}(N, \mathbf{k})$. One checks that that $\operatorname{Psdist}(N, \mathbf{k})$ is a torsor under the actions of $\operatorname{GTMD}(N, \mathbf{k})$ and $\operatorname{GRTMD}(N, \mathbf{k})$. The right action of $\operatorname{GRTMD}(N, \mathbf{k})$ on $\underline{\operatorname{Psdist}}(N, \mathbf{k})$ maps to the right action of $(\mathbb{Z} / N \mathbb{Z})^{\times} \times\left(\mathbf{k}^{\nu} \rtimes \mathbf{k}^{\times}\right)$on $(\mathbb{Z} / N \mathbb{Z}) \times\left(\mathbf{k}^{\nu} \rtimes \mathbf{k}\right)$. 
10.5. Distributivity of $\Psi_{\mathrm{KZ}}$. Let us denote by $\Psi_{\mathrm{KZ}}^{N}$ the element of $\operatorname{Pseudo}_{(-\overline{1}, 2 \pi \mathrm{i})}(N, \mathbb{C})$ constructed in Section 2.2.

Lemma 10.4. The morphism $\pi_{N N^{\prime}}: \operatorname{Pseudo}_{(-\overline{1}, 2 \pi \mathrm{i})}(N, \mathbb{C}) \rightarrow \operatorname{Pseudo}_{(-\overline{1}, 2 \pi \mathrm{i})}\left(N^{\prime}, \mathbb{C}\right)$ takes $\left(\Phi_{\mathrm{KZ}}, \Psi_{\mathrm{KZ}}^{N}\right)$ to $\left(\Phi_{\mathrm{KZ}}, e^{\log (d) b(0)} \Psi_{\mathrm{KZ}}^{N^{\prime}}\right)$. Moreover $\delta_{N N^{\prime}}\left(\Psi_{\mathrm{KZ}}^{N}\right)=\Psi_{\mathrm{KZ}}^{N^{\prime}}$, so $\left(\Phi_{\mathrm{KZ}}, \Psi_{\mathrm{KZ}}^{N}\right) \in$ Psdist $_{(-\overline{1}, 2 \pi i)}(N, \mathbb{C})$; its image in $\operatorname{Hom}(\operatorname{Div}(N), \mathbb{C})$ is the restriction of $\log$ to $\operatorname{Div}(N)$.

Proof. Let $H(z)$ be a solution of

$$
\frac{\mathrm{d} H}{\mathrm{~d} z}=\left(\frac{A}{z}+\sum_{a=0}^{N-1} \frac{b(a)}{z-\zeta_{N}^{a}}\right) H(z),
$$

where $\zeta_{N}$ is a primitive $N$ th root of 1 . Let $\bar{H}(z)$ be the image of $H(z)$ by $\pi_{N N^{\prime}}$. Then it follows from the identity $\sum_{k=0}^{d-1} 1 /\left(z-\zeta_{d}^{k}\right)=d z^{d-1} /\left(z^{d}-1\right)$ that $K(w):=\bar{H}\left(w^{1 / d}\right)$ satisfies

$$
\frac{\mathrm{d} \bar{K}}{\mathrm{~d} w}=\left(\frac{A^{\prime}}{w}+\sum_{a^{\prime}=0}^{N^{\prime}-1} \frac{b^{\prime}\left(a^{\prime}\right)}{w-\zeta_{N^{\prime}}^{a^{\prime}}}\right) \bar{K}(w)
$$

(we set $\zeta_{d}:=\zeta_{N}^{N^{\prime}}, \zeta_{N^{\prime}}:=\zeta_{N}^{d}$ ). The renormalized holonomy of $K(w)$ is $\Psi_{\mathrm{KZ}}^{N^{\prime}}$, hence the renormalized holonomy of $\bar{H}(z)$ is $e^{\log (d) b(0)} \Psi_{\mathrm{KZ}}^{N^{\prime}}$ (taking into account the change of variables). On the other hand, this is the image of $\Psi_{\mathrm{KZ}}^{N}$ by $\pi_{N N^{\prime}}$.

10.6. Surjectivity of $\operatorname{Psdist}(N, \mathbf{k}) \rightarrow(\mathbb{Z} / N \mathbb{Z})^{\times} \times\left(\mathbf{k}^{\nu} \rtimes \mathbf{k}^{\times}\right)$. One can use the torsor structure of $\operatorname{Psdist}(N, \mathbf{k})$ and its nonemptiness for $\mathbf{k}=\mathbb{C}$ to prove that $\operatorname{Psdist}(N, \mathbf{k}) \rightarrow(\mathbb{Z} / N \mathbb{Z})^{\times} \times \mathbf{k}^{\times}$is surjective. We will see in Theorem 11.6 that the morphism $\mathfrak{g r t m o}_{(\overline{1}, 1)}(N, \mathbf{k}) \rightarrow \mathbf{k}^{\nu}$ is surjective, which implies:

Proposition 10.5. The map $\operatorname{Psdist}(N, \mathbf{k}) \rightarrow(\mathbb{Z} / N \mathbb{Z})^{\times} \times\left(\mathbf{k}^{\nu} \rtimes \mathbf{k}^{\times}\right)$is surjective.

\section{Generators of $\mathfrak{g r t m}_{(\overline{1}, 1)}(N, \mathbb{C})$}

11.1. The element $(\lambda=-1, f=1)$ of GT takes a QTQBA $\left(A, m_{A}, \Delta_{A}, R, \Phi_{A}\right)$ to $A^{\prime}:=$ $\left(A, m_{A}, \Delta_{A},\left(R_{A}^{21}\right)^{-1}, \Phi_{A}\right)$; the element $(\lambda=-1, f=g=1)$ of GTM takes the QRA $\left(B, m_{B}, \Delta_{B}, E, \Psi_{B}\right)$ over $A$ to the QRA $B^{\prime}:=\left(B, m_{B}, \Delta_{B}, E^{-1}, \Psi_{B}\right)$ over $A^{\prime}$.

It follows that there is a unique map $\underline{\mathbf{A s s o c}}_{\lambda}(\mathbf{k}) \rightarrow \underline{\mathbf{A s s o c}}_{-\lambda}(\mathbf{k})$ taking $\Phi$ to itself, and a unique morphism $\underline{\text { Psdist }}_{(\alpha, \lambda)}(N, \mathbf{k}) \rightarrow \underline{\text { Psdist }}_{(-\alpha,-\lambda)}(N, \mathbf{k})$, taking $(\Phi, \Psi)$ to itself. Composing the first map with the action of $-1 \in \mathbf{k}^{\times} \subset \operatorname{GRT}(\mathbf{k})$, we get a permutation of $\underline{\mathbf{A s s o c}}_{\lambda}(\mathbf{k})$, taking $\Phi(A, B)$ to $\Phi(-A,-B)$. Composing the second map with the action of $(-\overline{1},-1) \in$ $(\mathbb{Z} / N \mathbb{Z})^{\times} \times \mathbf{k}^{\times} \subset \operatorname{GRTMD}(N, \mathbf{k})$, we get a permutation of $\underline{\text { Psdist }}_{(\alpha, \lambda)}(N, \mathbf{k})$, taking

$$
(\Phi(U, V), \Psi(A \mid b(0), \ldots, b(N-1))) \text { to }(\Phi(-U,-V), \Psi(-A \mid-b(0), \ldots,-b(1-N))) .
$$

In particular, there is a unique element $\left(g_{\mathrm{KZ}}, h_{\mathrm{KZ}}\right) \in \operatorname{GRTMD}_{(\overline{1}, 1)}(N, \mathbb{C})$, such that

$$
\left(\Phi_{\mathrm{KZ}}(-U,-V), \Psi_{\mathrm{KZ}}(-A-b(0), \ldots,-b(1-N))\right)=\left(\Phi_{\mathrm{KZ}}, \Psi_{\mathrm{KZ}}\right) *\left(g_{\mathrm{KZ}}, h_{\mathrm{KZ}}\right) .
$$

Let $\log : \operatorname{GRT}_{1}(\mathbb{C}) \rightarrow \mathfrak{g r t}_{1}(\mathbb{C})$ be the logarithmic map. Set $\varphi:=\log \left(g_{\mathrm{KZ}}\right)$. According to [Dr2], for $n$ odd $\geq 3$, the degree $n$ component $\varphi_{n}$ of $\varphi$ is a generator of $\mathfrak{g r t}_{1}(\mathbb{C})$ (i.e., its class in $\mathfrak{g r t}_{1}(\mathbb{C}) /\left[\mathfrak{g r t}_{1}(\mathbb{C}), \mathfrak{g r t}_{1}(\mathbb{C})\right]$ is nonzero).

Set $(\varphi, \psi):=\log \left(g_{\mathrm{KZ}}, h_{\mathrm{KZ}}\right)$, so $(\varphi, \psi) \in \mathfrak{g r t m}_{(\overline{1}, 1)}(N, \mathbb{C})$. When $n$ is odd $\geq 3$, the degree $n$ component $\left(\varphi_{n}, \psi_{n}\right)$ of $(\varphi, \psi)$ is a generator of $\mathfrak{g r t m o}_{(\overline{1}, 1)}(N, \mathbb{C})$, and is a preimage of $\varphi_{n}$. So the image of $\mathfrak{g r t m} \mathfrak{d}_{(\overline{1}, 1)}(N, \mathbb{C}) \rightarrow \mathfrak{g r t}_{1}(\mathbb{C})$ contains the subalgebra generated by the $\varphi_{n}$. 
11.2. To obtain more information of $\left(\varphi_{n}, \psi_{n}\right) \in \mathfrak{g r t m}_{(\overline{1}, 1)}(N, \mathbb{C})$, we study the expansion of $\log \left(\Psi_{\mathrm{KZ}}\right)$. When $A$ and the $b[\zeta], \zeta \in \mu_{N}(\mathbb{C})$ commute, the renormalized holonomy of $(64)$ is equal to

$$
\exp \left(\sum_{\zeta \in \mu_{N}(\mathbb{C}), \zeta \neq 1} \log \left(\zeta^{-1}-1\right) b[\zeta]\right) .
$$

Let $\hat{\mathfrak{f}}_{N+1}:=\hat{\mathfrak{f}}\left(A, b[\zeta], \zeta \in \mu_{N}(\mathbb{C})\right)$. Then $\log \left(\Psi_{\mathrm{KZ}}\right) \in \hat{\mathfrak{f}}_{N+1}$, and its image in $\hat{\mathfrak{f}}_{N+1} /\left[\hat{\mathfrak{f}}_{N+1}, \hat{\mathfrak{f}}_{N+1}\right]$ is $\sum_{\zeta \in \mu_{N}(\mathbb{C}), \zeta \neq 1} \log \left(\zeta^{-1}-1\right) b[\zeta]$. It follows that $\log \left(\Psi_{\mathrm{KZ}}\right) \in \hat{\mathfrak{f}}_{N+1}^{A, b[1]}$, where the latter space is the topological sum of all finely homogeneous components of $\hat{\mathfrak{f}}_{N+1}$, except $\mathbb{C} A$ and $\mathbb{C} b[1]$ (we define a fine degree in $\mathbb{N} \tilde{\mu}_{N}=\mathbb{N}\left(\mu_{n}(\mathbb{C}) \cup\{0\}\right)$ by $\operatorname{deg}(A)=0, \operatorname{deg}(b[\zeta])=\zeta$; see Appendix A).

By Lazard elimination (see $[\operatorname{Re}]), \mathfrak{q}:=\hat{\mathfrak{f}}_{N+1}^{A, b[1]}$ is freely generated by the $U_{k, l}(k, l \geq 1)$ and the $V_{k, l, \zeta}\left(k, l \geq 0, \zeta \in \mu_{N}(\mathbb{C}), \zeta \neq 1\right)$, where

$$
U_{k, l}=\operatorname{ad}(A)^{k-1} \operatorname{ad}(b[1])^{l-1}([A, b[1]]), \quad V_{k, l, \zeta}=\operatorname{ad}(A)^{k} \operatorname{ad}(b[1])^{l}(b[\zeta]) .
$$

Let $V(z):=(1-x)^{-b[1]} x^{-A} H(z)$, where $H(z)$ is defined by (64). Then

$$
\frac{\mathrm{d}}{\mathrm{d} z} V(z)=\left((1-z)^{-b[1]} z^{-A}\left(\sum_{\zeta \in \mu_{N}(\mathbb{C})} \frac{b[\zeta]}{z-\zeta}\right) z^{A}(1-z)^{b[1]}-\frac{b[1]}{z-1}\right) V(z)
$$

Therefore the image of $\log \left(\Psi_{\mathrm{KZ}}\right)$ in $\mathfrak{q}^{\text {ab }}:=\mathfrak{q} /[\mathfrak{q}, \mathfrak{q}]$ is

$\sum_{k, l \geq 1} \frac{1}{k !(l-1) !} \int_{0}^{1}(-\log z)^{k}(-\log (1-z))^{l-1} \frac{\mathrm{d} z}{z-1} \bar{U}_{k, l}+\sum_{k, l \geq 0} \frac{1}{k ! l !} \int_{0}^{1}(-\log z)^{k}(-\log (1-z))^{l} \frac{\mathrm{d} z}{z-\zeta} \bar{V}_{k, l, \zeta}$

$\left(x \mapsto \bar{x}\right.$ is the projection $\left.\mathfrak{q} \rightarrow \mathfrak{q}^{\text {ab }}\right)$.

11.3. The infinitesimal action of $\mathfrak{g r t m} \mathfrak{d}_{(\overline{1}, 1)}(N, \mathbf{k})$ on $\underline{\operatorname{Psdist}}(N, \mathbf{k})$ is given by

$$
\delta_{(\varphi, \psi)}(\Phi, \Psi)=\left(\Phi \varphi+D_{\varphi}(\Phi), \Psi \psi+\bar{D}_{\psi}(\Psi)\right),
$$

where $D_{\varphi}, \bar{D}_{\psi}$ are given by (44) and (45).

Define a bracket on $\hat{\mathfrak{f}}_{2} \oplus \hat{\mathfrak{f}}_{N+1}$ by formulas (41), (42) and (43); this is a Lie bracket. Then $\mathfrak{g r t m o} \mathfrak{d}_{1}(N, \mathbf{k}) \subset \hat{\mathfrak{f}}_{2} \oplus \hat{\mathfrak{f}}_{N+1}$ is an inclusion of Lie algebras. As in [Dr2], let $\mathfrak{p}:=\hat{\mathfrak{f}}_{2}^{A, B} \subset \hat{\mathfrak{f}}_{2}$ be the topological sum of all homogeneous components of degree $>1$.

If $\alpha \geq 1$, denote by $\mathfrak{g r t m}_{(\overline{1}, 1)}(N, \mathbf{k})_{\alpha}$ the degree $\alpha$ part of $\mathfrak{g r t m}_{(\overline{1}, 1)}(N, \mathbf{k})$. Set

$$
\mathfrak{g r t m}_{(\overline{1}, 1)}(N, \mathbf{k})_{1}^{+}:=\mathfrak{g r t m}_{(\overline{1}, 1)}(N, \mathbf{k})_{1} \cap\left(\bigoplus_{a \in \mathbb{Z} / N \mathbb{Z}, a \neq 0} \mathbb{C}(0, b(a))\right),
$$

and

$$
\mathfrak{g r t m} \mathfrak{d}_{(\overline{1}, 1)}(N, \mathbf{k})^{+}:=\mathfrak{g r t m}_{(\overline{1}, 1)}(N, \mathbf{k})_{1}^{+} \oplus\left(\widehat{\bigoplus}_{\alpha \geq 2} \mathfrak{g r t m} \mathfrak{d}_{(\overline{1}, 1)}(N, \mathbf{k})_{\alpha}\right) .
$$

Then $\mathfrak{g r t m}_{(\overline{1}, 1)}(N, \mathbf{k})=\mathfrak{g r t m}_{(\overline{1}, 1)}(N, \mathbf{k})^{+} \oplus \mathbf{k}(0, b(0))$; the first summand is a Lie subalgebra and the second summand is central.

Lemma 11.1. The linear map $\hat{\mathfrak{f}}_{N+1} \otimes \hat{\mathfrak{f}}_{N+1} \rightarrow \hat{\mathfrak{f}}_{N+1}, \psi_{1} \otimes \psi_{2} \mapsto \bar{D}_{\psi_{1}}\left(\psi_{2}\right)$, restricts to a linear map $\mathfrak{q} \otimes \mathfrak{q} \rightarrow \mathfrak{q}$. The latter map induces a map $\mathfrak{q}^{\mathrm{ab}} \otimes \mathfrak{q}^{\mathrm{ab}} \rightarrow \mathfrak{q}^{\mathrm{ab}}$.

Proof. Set $\mathfrak{q}_{1}:=\oplus_{a \in \mathbb{Z} / N \mathbb{Z}, a \neq 0} \mathbf{k} b(a)$, then $\mathfrak{q}=\mathfrak{q}_{1} \oplus\left(\widehat{\oplus}_{\alpha \geq 2}\left(\mathfrak{f}_{N+1}\right)_{\alpha}\right)$. If $\psi_{1}$ and $\psi_{2}$ are homogeneous in $\mathfrak{f}_{N+1}$, then $\operatorname{deg}\left(\bar{D}_{\psi_{1}}\left(\psi_{1}\right)\right)=\operatorname{deg}\left(\psi_{1}\right)+\operatorname{deg}\left(\psi_{2}\right) \geq 2$, hence $\bar{D}_{\psi_{1}}\left(\psi_{2}\right) \in \mathfrak{q}$. If now $\psi_{1}=\left[\psi^{\prime}, \psi^{\prime \prime}\right]$, where $\psi^{\prime}, \psi^{\prime \prime} \in \mathfrak{q}$, then $\bar{D}_{\psi_{1}}\left(\psi_{2}\right)=\left[\bar{D}_{\psi_{1}}\left(\psi^{\prime}\right), \psi^{\prime \prime}\right]+\left[\psi^{\prime}, \bar{D}_{\psi_{1}}\left(\psi^{\prime \prime}\right)\right] \in[\mathfrak{q}, \mathfrak{q}]$. Hence $\mathfrak{q} \otimes[\mathfrak{q}, \mathfrak{q}]$ maps to $[\mathfrak{q}, \mathfrak{q}]$.

Let us now show that $[\mathfrak{q}, \mathfrak{q}] \otimes \mathfrak{q}$ maps to $[\mathfrak{q}, \mathfrak{q}]$. For this, we will show that if $\psi \in[\mathfrak{q}, \mathfrak{q}]$ and $\psi \in \mathfrak{f}_{N+1}$, then $\bar{D}_{\psi}\left(\psi^{\prime}\right) \in[\mathfrak{q}, \mathfrak{q}]$. It suffices to show this when $\psi^{\prime}=A$ or $\psi^{\prime}=b(a), a \in \mathbb{Z} / N \mathbb{Z}$. 
BENJAMIN ENRIQUEZ

We have $\bar{D}_{\psi}(b(0))=0$. [q, $\left.\mathfrak{q}\right]$ is an ideal in $\hat{\mathfrak{f}}_{N+1}$, hence $\bar{D}_{\psi}(A)=[\psi, A] \in[\mathfrak{q}, \mathfrak{q}]$. For the same reason, $\bar{D}_{\psi}(b(a)) \in[\mathfrak{q}, \mathfrak{q}]$. Hence it remains to show that $[\psi(A \mid b(a), \ldots, b(a+N-1)), b(a)] \in$ $[\mathfrak{q}, \mathfrak{q}]$ when $a \neq 0$. This follows from the facts that $b(a) \in \mathfrak{q}, \psi$ has degree $\geq 2$, and for any $d \geq 2$, the degree $d$ part of $\mathfrak{q}$ is stable under the automorphism $A \mapsto A, b(\alpha) \mapsto b(a+\alpha)$.

Lemma 11.2. Let us denote by $\psi_{1} \otimes \psi_{2} \mapsto \bar{D}_{\psi_{1}}\left(\psi_{2}\right)$ the above map $\mathfrak{q}^{\text {ab }} \otimes \mathfrak{q}^{\text {ab }} \rightarrow \mathfrak{q}^{\text {ab }}$. We have

$$
\begin{gathered}
\bar{D}_{\bar{U}_{k l}}\left(\bar{U}_{k^{\prime} l^{\prime}}\right)=\bar{U}_{k+k^{\prime}, l+l^{\prime}}, \bar{D}_{\bar{U}_{k l}}\left(\bar{V}_{k^{\prime}, l^{\prime}, \zeta}\right)=\bar{V}_{k+k^{\prime}, l+l^{\prime}, \zeta}, \\
\bar{D}_{\bar{V}_{k, l, \zeta}}\left(\bar{U}_{k^{\prime} l^{\prime}}\right)=\bar{V}_{k+k^{\prime}, l+l^{\prime}, \zeta}, \bar{D}_{\bar{V}_{k, l, \zeta}}\left(\bar{V}_{k^{\prime}, l^{\prime}, \zeta^{\prime}}\right)=0
\end{gathered}
$$

if $\left(k, l, \zeta^{\prime}\right) \neq\left(0,0, \zeta^{-1}\right)$, and $\bar{D}_{\bar{V}_{0,0, \zeta}}\left(\bar{V}_{k^{\prime}, l^{\prime}, \zeta^{-1}}\right)=\bar{V}_{k^{\prime}, l^{\prime}+1, \zeta^{-1}}$. by

In the same way, the map $\mathfrak{p}^{\mathrm{ab}} \otimes \mathfrak{p}^{\mathrm{ab}} \rightarrow \mathfrak{p}^{\mathrm{ab}}$, which we denote by $\varphi_{1} \otimes \varphi_{2} \mapsto D_{\varphi_{1}}\left(\varphi_{2}\right)$ is given

$$
D_{\bar{A}_{k l}}\left(\bar{A}_{k^{\prime} l^{\prime}}\right)=\bar{A}_{k+k^{\prime}, l+l^{\prime}},
$$

where $A_{k l}=\operatorname{ad}(A)^{k-1} \operatorname{ad}(B)^{l-1}([A, B]), k, l \geq 1$ are the free generators of $\mathfrak{p}$, and $\bar{A}_{k l}$ are their images in $\mathfrak{p}^{\mathrm{ab}}$.

Let us set $\bar{\Psi}_{\mathrm{KZ}}:=\Psi_{\mathrm{KZ}}(-A \mid-b(0), \ldots,-b(1-N))$. Recall that $\psi$ is uniquely determined by $\bar{\Psi}_{\mathrm{KZ}}=\Psi_{\mathrm{KZ}} * \operatorname{Exp}(\psi)$. Set $\Psi(t):=\Psi_{\mathrm{KZ}} * \operatorname{Exp}(t \psi)$. Then $\log \Psi(t) \in \mathfrak{q}$; we decompose its image in $\mathfrak{q}^{\text {ab }}$ as follows

$$
[\log \Psi(t)]=\sum_{k, l \geq 1} c_{k l}(t) \bar{U}_{k l}+\sum_{\zeta \in \mu_{N}(\mathbb{C}), \zeta \neq 1} \sum_{k, l \geq 0} d_{k, l, \zeta}(t) \bar{V}_{k, l, \zeta} .
$$

We also decompose the image of $\psi$ in $\mathfrak{q}^{\text {ab }}$ as

$$
[\psi]=\sum_{k, l \geq 1} \varphi_{k l} \bar{U}_{k l}+\sum_{\zeta \in \mu_{N}(\mathbb{C}), \zeta \neq 1} \sum_{k, l \geq 0} \psi_{k, l, \zeta} \bar{V}_{k, l, \zeta} .
$$

Then $\psi_{0,0, \zeta}=-\log (\zeta-1)\left(\zeta^{-1}-1\right)$ when $\zeta \neq 1$, and $\psi_{0,0,1}=0$.

We will set $\varphi(u, v)=\sum_{k, l \geq 1} \varphi_{k l} u^{k} v^{l}, \psi_{\zeta}(u, v)=\sum_{k, l>0} \psi_{k, l, \zeta} u^{k} v^{l}$, and define $c(u, v)$ and $d_{\zeta}(u, v)$ similarly. According to $(55)$, we have $\Psi^{\prime}(t)=\Psi(t) \psi+\bar{D}_{\psi}(\Psi(t))$, which according to Lemma 11.2 implies

$$
\begin{gathered}
\frac{\partial}{\partial t} c(u, v, t)=\varphi(u, v)(c(u, v, t)+1) \\
\frac{\partial}{\partial t} d_{\zeta}(u, v, t)=\psi_{\zeta}(u, v)(1+c(u, v, t))+\left(\varphi(u, v)-\psi_{0,0, \zeta^{-1} v}\right) d_{\zeta}(u, v, t) .
\end{gathered}
$$

According to [Dr2], we have

$$
1+c(u, v, 0)=\frac{\Gamma(1-u) \Gamma(1-v)}{\Gamma(1-u-v)}=\exp \left(\sum_{n \geq 2} \frac{\zeta(n)}{n}\left(u^{n}+v^{n}-(u+v)^{n}\right)\right),
$$

$c(u, v, 1)=c(-u,-v, 0)$, and since $1+c(u, v, t)=(1+c(u, v, 0)) e^{t \varphi(u, v)}$, we get

$$
\begin{aligned}
\varphi(u, v) & =\log \frac{\Gamma(1+u)}{\Gamma(1-u)}+\log \frac{\Gamma(1+v)}{\Gamma(1-v)}-\log \frac{\Gamma(1+u+v)}{\Gamma(1-u-v)} \\
& =-2 \sum_{n \text { odd } \geq 3} \frac{\zeta(n)}{n}\left(u^{n}+v^{n}-(u+v)^{n}\right) .
\end{aligned}
$$

On the other hand, we have

$$
d_{\zeta}(u, v, 0)=\int_{0}^{1} z^{-u}(1-z)^{-v} \frac{d z}{z-\zeta}
$$


and $d_{\zeta}(u, v, 1)=-d_{\zeta^{-1}}(-u,-v, 0)$. Since

$$
d_{\zeta}(u, v, t) e^{-t\left(\varphi(u, v)-\psi_{0,0, \zeta^{-1}} v\right)}=d_{\zeta}(u, v, 0)+\psi_{\zeta}(u, v)(1+c(u, v, 0)) \frac{e^{t \psi_{0,0, \zeta^{-1}} v}-1}{\psi_{0,0, \zeta^{-1}} v}
$$

we get

$$
\psi_{\zeta}(u, v)=\alpha_{\zeta}(u, v)+\alpha_{\zeta^{-1}}(-u,-v)
$$

where

$$
\alpha_{\zeta}(u, v)=\frac{v \log \left((1-\zeta)\left(1-\zeta^{-1}\right)\right)}{\left((1-\zeta)\left(1-\zeta^{-1}\right)\right)^{-v}-1} \frac{\Gamma(1-u-v)}{\Gamma(1-u) \Gamma(1-v)} \int_{0}^{1} \frac{z^{-u}(1-z)^{-v}}{z-\zeta} d z .
$$

11.4. Recall that $\mathfrak{q}^{\text {ab }}$ is spanned by the $\bar{V}_{k, l, \zeta}, k, l \geq 0, \zeta \in \mu_{N}(\mathbb{C}), \zeta \neq 1$. Let us denote by $\mathfrak{q}_{l>0}^{\text {ab }}$ the span of all $\bar{V}_{k, l, \zeta}$ where $l>0$; we denote the quotient $\mathfrak{q}^{\mathrm{ab}} / \mathfrak{q}_{l>0}^{\mathrm{ab}}$ by $\mathfrak{q}_{l=0}^{\mathrm{ab}}$.

Lemma 11.3. The image of the sequence of maps

$$
\left[\mathfrak{g r t m} \mathfrak{d}_{(\overline{1}, 1)}(N, \mathbf{k}), \mathfrak{g r t m} \mathfrak{d}_{(\overline{1}, 1)}(N, \mathbf{k})\right] \subset \mathfrak{g r t m} \mathfrak{d}_{(\overline{1}, 1)}(N, \mathbf{k})^{+} \subset \mathfrak{q} \rightarrow \mathfrak{q}^{\text {ab }}
$$

is contained in $\mathfrak{q}_{l>0}^{\mathrm{ab}}$, so we have a map

$$
\mathfrak{g r t m}_{(\overline{1}, 1)}(N, \mathbf{k})^{+} /\left[\mathfrak{g r t m} \mathfrak{d}_{(\overline{1}, 1)}(N, \mathbf{k}), \mathfrak{g r t m} \mathfrak{d}_{(\overline{1}, 1)}(N, \mathbf{k})\right] \rightarrow \mathfrak{q}_{l=0}^{\mathrm{ab}} .
$$

Proof. Let us denote by $\mathfrak{q}_{l>0}$ the part of $\mathfrak{q}$ of positive relative degree w.r.t. $b(0)$. Then $[\mathfrak{q}, \mathfrak{q}]+\mathfrak{q}_{l>0}$ is an ideal of $\mathfrak{q}$ (for the bracket $[-,-]$ ). We will show that

$$
\langle\mathfrak{q}, \mathfrak{q}\rangle \subset[\mathfrak{q}, \mathfrak{q}]+\mathfrak{q}_{l>0} .
$$

We will use the formula

$$
\left\langle\psi_{1}, \psi_{2}\right\rangle=-\left[\psi_{1}, \psi_{2}\right]+\bar{D}_{\psi_{2}}^{\prime}\left(\psi_{1}\right)-\bar{D}_{\psi_{1}}^{\prime}\left(\psi_{2}\right),
$$

where $\bar{D}_{\psi}^{\prime}$ is the derivation such that $\bar{D}_{\psi}^{\prime}(A)=0, \bar{D}_{\psi}^{\prime}(b(a))=-[\psi(A \mid b(a), \ldots, b(a+N-$ 1)), $b(a)]$.

If $\psi \in \mathfrak{q}$ has degree $\geq 2$ and $a \in \mathbb{Z} / N \mathbb{Z}$, then $\bar{D}_{\psi}^{\prime}(b(a)) \in[\mathfrak{q}, \mathfrak{q}]+\mathfrak{q}_{l>0}$. Therefore, if $\psi^{\prime} \in \mathfrak{q}$ is arbitrary, then $\bar{D}_{\psi}^{\prime}\left(\psi^{\prime}\right) \in[\mathfrak{q}, \mathfrak{q}]+\mathfrak{q}_{l>0}$. If $\psi$ and $\psi^{\prime} \in \mathfrak{q}$ both have degree $\geq 2$, we then get $\left\langle\psi, \psi^{\prime}\right\rangle \in[\mathfrak{q}, \mathfrak{q}]+\mathfrak{q}_{l>0}$.

Let again $\psi \in \mathfrak{q}$ be of degree $\geq 2$ and $a \in \mathbb{Z} / N \mathbb{Z}-\{0\}$. If $a^{\prime} \in \mathbb{Z} / N \mathbb{Z}$, then $\bar{D}_{b(a)}^{\prime}\left(b\left(a^{\prime}\right)\right) \in$ $[\mathfrak{q}, \mathfrak{q}]+\mathfrak{q}_{l>0}$. Therefore if $\psi \in \mathfrak{q}$, we have $\bar{D}_{b(a)}^{\prime}(\psi) \in[\mathfrak{q}, \mathfrak{q}]+\mathfrak{q}_{l>0}$. Since $\bar{D}_{\psi}^{\prime}(b(a)) \in[\mathfrak{q}, \mathfrak{q}]$, and $[b(a), \psi] \in[\mathfrak{q}, \mathfrak{q}]$, we get $\langle b(a), \psi\rangle \in[\mathfrak{q}, \mathfrak{q}]+\mathfrak{q}_{l>0}$. This implies that $\langle b(a)+b(-a), \psi\rangle \in[\mathfrak{q}, \mathfrak{q}]+\mathfrak{q}_{l>0}$.

Finally, if $a, a^{\prime} \neq 0$ and $a+a^{\prime} \neq 0$, we have $\left\langle b(a), b\left(a^{\prime}\right)\right\rangle=\left[b\left(a+a^{\prime}\right), b\left(a^{\prime}\right)-b(a)\right]-\left[b(a), b\left(a^{\prime}\right)\right] \in$ $[\mathfrak{q}, \mathfrak{q}]$, which implies that $\left\langle b(a)+b(-a), b\left(a^{\prime}\right)+b\left(-a^{\prime}\right)\right\rangle \in[\mathfrak{q}, \mathfrak{q}]$.

This proves (56) and therefore the lemma.

11.5. Generators of $\mathfrak{g r t m}_{(\overline{1}, 1)}(N, \mathbb{C})^{+}$. We will set

$$
\mathfrak{g r t m}_{(\overline{1}, 1)}(N, \mathbf{k})^{+, \mathrm{ab}}:=\mathfrak{g r t m}_{(\overline{1}, 1)}(N, \mathbf{k})^{+} /\left[\mathfrak{g r t m} \mathfrak{d}_{(\overline{1}, 1)}(N, \mathbf{k}), \mathfrak{g r t m} \mathfrak{d}_{(\overline{1}, 1)}(N, \mathbf{k})\right]
$$

and denote by $\mathfrak{g r t m} \mathfrak{d}_{(\overline{1}, 1)}(N, \mathbf{k})_{n}^{+, \text {ab }}$ the degree $n$ part of this space.

We have a linear map $\mathfrak{g r t m}_{(\overline{1}, 1)}(N, \mathbf{k})^{+, \mathrm{ab}} \rightarrow \mathfrak{p}^{\mathrm{ab}} \oplus \mathfrak{q}_{l=0}^{\mathrm{ab}}$. The degree $n$ part of $\mathfrak{p}^{\mathrm{ab}} \oplus \mathfrak{q}_{l=0}^{\mathrm{ab}}$ is spanned by the $\left(\bar{A}_{k l}, 0\right)$, where $k, l \geq 1, k+l=n$, and the $\left(0, \bar{V}_{n-1,0, \zeta}\right), \zeta \in \mu_{N}(\mathbb{C}), \zeta \neq 1$. The image of $\left(\varphi_{n}, \psi_{n}\right)$ in $\mathfrak{p}^{\mathrm{ab}} \oplus \mathfrak{q}_{l=0}^{\mathrm{ab}}$ is equal to

$$
\left(0, \sum_{\zeta \in \mu_{N}(\mathbb{C}), \zeta \neq 1}-2 \log |1-\zeta| \bar{V}_{0,0, \zeta}\right)
$$


if $n=1$, and to

$$
\left(\frac{\zeta(n)}{n}\left(1+(-1)^{n+1}\right) \sum_{k, l \geq 1, k+l=n}\left(\begin{array}{l}
n \\
k
\end{array}\right) \bar{A}_{k l}, \sum_{\zeta \in \mu_{N}(\mathbb{C}), \zeta \neq 1}\left(Z\left(n, \zeta^{-1}\right)+(-1)^{n+1} Z(n, \zeta)\right) \bar{V}_{n-1,0, \zeta}\right),
$$

where $Z(n, \zeta)=\sum_{k \geq 1} \zeta^{k} / k^{n}$ (so $\left.Z(n, \zeta)=\operatorname{Li}_{n}(\zeta)\right)$ for $n \geq 2$. The images of $\left(\varphi_{n}, \psi_{n}\right)$ in $\mathfrak{q}_{l=0}^{\text {ab }}$ can be written uniformly in $n$ since $Z(1, z)=-\log (1-z)$.

Recall that $(\mathbb{Z} / N \mathbb{Z})^{\times}$acts on $\mathfrak{g r t m} \mathfrak{d}_{(\overline{1}, 1)}(N, \mathbb{C})$. If $k \in(\mathbb{Z} / N \mathbb{Z})^{\times}$, then the vectors obtained from (57), (58) by replacing $\zeta$ by $\zeta^{k}$ in $\log |1-\zeta|$ and $Z\left(n, \zeta^{ \pm 1}\right)$, also belong to the image of $\mathfrak{g r t m}_{(\overline{1}, 1)}(N, \mathbb{C})^{+, \mathrm{ab}} \rightarrow \mathfrak{p}^{\mathrm{ab}} \oplus \mathfrak{q}_{l=0}^{\mathrm{ab}}$.

Let $\mathbb{C} \mu_{N}(\mathbb{C})$ be the complex vector space with basis $[\zeta]$, where $\zeta \in \mu_{N}(\mathbb{C})$. Set for $n \geq 2$

$$
V_{n}:=\operatorname{Span}_{\mathbb{C}}\left\{\sum_{\zeta \in \mu_{N}(\mathbb{C})}\left((-1)^{n+1} Z\left(n, \zeta^{k}\right)+Z\left(n, \zeta^{-k}\right)\right)[\zeta], k \in(\mathbb{Z} / N \mathbb{Z})^{\times}\right\} \subset \mathbb{C} \mu_{N}(\mathbb{C})
$$

and

$$
V_{1}:=\operatorname{Span}_{\mathbb{C}}\left\{\sum_{\zeta \in \mu_{N}(\mathbb{C}), \zeta \neq 1}-2 \log \left|1-\zeta^{k}\right|[\zeta], k \in(\mathbb{Z} / N \mathbb{Z})^{\times}\right\} \subset \mathbb{C} \mu_{N}(\mathbb{C}) .
$$

Then for any $n \geq 1, \operatorname{ch} \mathfrak{g r t m}_{(\overline{1}, 1)}(N, \mathbb{C})_{n}^{+, \text {ab }} \geq \operatorname{ch}\left(V_{n}\right)$, where ch means the character as $(\mathbb{Z} / N \mathbb{Z})^{\times}$-module.

Computing $\operatorname{ch}\left(V_{n}\right)$ means decomposing this space in isotypic components under the action of $(\mathbb{Z} / N \mathbb{Z})^{\times}$. We first decompose $\mathbb{C} \mu_{N}(\mathbb{C})$.

Let $\chi:(\mathbb{Z} / N \mathbb{Z})^{\times} \rightarrow \mathbb{C}^{\times}$be a character. The set $D_{\chi}:=\{d \mid d$ divides $N$ and $\chi$ factors through $\left.(\mathbb{Z} / N \mathbb{Z})^{\times} \rightarrow(\mathbb{Z} / d \mathbb{Z})^{\times}\right\}$is closed under the operation of taking greatest common divisors. The smallest element $f_{\chi}$ in $D_{\chi}$ is called the conductor of $\chi$. The resulting character $\chi^{\prime}:\left(\mathbb{Z} / f_{\chi} \mathbb{Z}\right)^{\times} \rightarrow$ $\mathbb{C}^{\times}$has conductor 1 .

Let us denote by $\operatorname{Prim}_{d}$ the set of primitive $d$ th roots of 1 in $\mathbb{C}$. Then Prim $d$ is a torsor under the action of $(\mathbb{Z} / d \mathbb{Z})^{\times}$. It follows that

$$
\mathbb{C} \operatorname{Prim}_{d}=\bigoplus_{\chi \text { character of }(\mathbb{Z} / d \mathbb{Z})^{\times}}\left(\mathbb{C} \operatorname{Prim}_{d}\right)^{\chi},
$$

and each summand is 1-dimensional, spanned by $\sum_{a \in(\mathbb{Z} / d \mathbb{Z})^{\times}} \chi(a)^{-1}\left[\zeta_{d}^{a}\right]$. We therefore obtain

$$
\mathbb{C} \mu_{N}(\mathbb{C})=\bigoplus_{\chi \text { character of }(\mathbb{Z} / N \mathbb{Z})^{\times}} \mathbb{C} \mu_{N}(\mathbb{C})^{\chi},
$$

where

$$
\mathbb{C} \mu_{N}(\mathbb{C})^{\chi}=\bigoplus_{d \text { such that } f_{\chi}|d| N}\left(\mathbb{C} \operatorname{Prim}_{d}\right)^{\chi^{\prime} \circ \pi_{\chi, d}},
$$

$\chi^{\prime}$ is the character of $\left(\mathbb{Z} / f_{\chi} \mathbb{Z}\right)^{\times}$obtained from $\chi$ and $\pi_{\chi, d}:(\mathbb{Z} / d \mathbb{Z})^{\times} \rightarrow\left(\mathbb{Z} / f_{\chi} \mathbb{Z}\right)^{\times}$is the natural projection.

Since $V_{n}$ is $(\mathbb{Z} / N \mathbb{Z})^{\times}$-invariant, it is graded w.r.t. the decomposition (59). Let us compute its graded components.

The isotypic component corresponding to the character $\chi$ is spanned by

$$
\begin{aligned}
v_{\chi} & :=\sum_{\zeta \in \mu_{N}(\mathbb{C})}\left(\sum_{k \in(\mathbb{Z} / N \mathbb{Z})^{\times}} \chi(k)\left((-1)^{n+1} Z\left(n, \zeta^{k}\right)+Z\left(n, \zeta^{-k}\right)\right)\right)[\zeta] \\
& =\left(\chi(-1)+(-1)^{n+1}\right) \sum_{\zeta \in \mu_{N}(\mathbb{C})}\left(\sum_{k \in(\mathbb{Z} / N \mathbb{Z})^{\times}} \chi(k) Z\left(n, \zeta^{k}\right)\right)[\zeta] ;
\end{aligned}
$$


here we adopt the convention that $Z(1, \zeta)=-\log (1-\zeta)$ if $\zeta \neq 1$ and $Z(1,1)=0$. A character is called even (resp., odd) if $\chi(-1)=1$ (resp., -1 ), so odd (resp., even) characters do not contribute if $n$ is odd (resp., even).

Proposition 11.4. If $n$ and $\chi$ have opposite parity, then $v_{\chi} \neq 0$.

Proof. Assume that $n>1$. The coefficient of $\left[\zeta_{N}\right]$ is equal to

$$
2(-1)^{n+1} \sum_{k \in(\mathbb{Z} / N \mathbb{Z})^{\times}} \chi(k) Z\left(n, \zeta_{N}^{k}\right)=2(-1)^{n+1} \sum_{a \geq 1} \frac{1}{a^{n}}\left(\sum_{k \in(\mathbb{Z} / N \mathbb{Z})^{\times}} \chi(k) \zeta_{N}^{k a}\right) .
$$

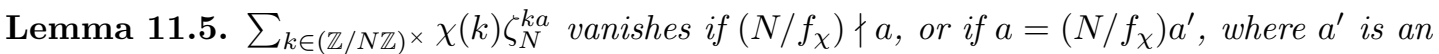
integer such that $\left(a^{\prime}, f_{\chi}\right) \neq 1$. If $a=\left(N / f_{\chi}\right) a^{\prime}$ where $a^{\prime}$ is an integer such that $\left(a^{\prime}, f_{\chi}\right)=1$, then

$$
\sum_{k \in(\mathbb{Z} / N \mathbb{Z})^{\times}} \chi(k) \zeta_{N}^{k a}=\left(N / f_{\chi}\right) \tau(\chi) \chi^{-1}\left(\left[a^{\prime}\right]\right)
$$

where $\left[a^{\prime}\right]$ is the class of $a^{\prime}$ in $\left(\mathbb{Z} / f_{\chi} \mathbb{Z}\right)^{\times}$, and $\tau(\chi)$ is the Gauss sum $\tau(\chi)=\sum_{l \in\left(\mathbb{Z} / f_{\chi} \mathbb{Z}\right) \times} \chi(l) \zeta_{f}^{l}$.

Proof of Lemma. We have

$$
\sum_{k \in(\mathbb{Z} / N \mathbb{Z})^{\times}} \chi(k) \zeta_{N}^{k a}=\sum_{l \in\left[0, f_{\chi}-1\right],\left(l, f_{\chi}\right)=1} \chi(l) \zeta_{N}^{l a}\left(1+\zeta_{N}^{f_{\chi} a}+\cdots+\zeta_{N}^{\left(\frac{N}{f_{\chi}}-1\right) f_{\chi} a}\right),
$$

so the 1.h.s. is zero if $N \nmid f_{\chi} a$, and is equal to $\left(N / f_{\chi}\right) \sum_{l \in\left[0, f_{\chi}-1\right],\left(l, f_{\chi}\right)=1} \chi(l) \zeta_{N}^{l a}$ otherwise.

Let us place ourselves in the last situation, and let $a=\left(N / f_{\chi}\right) a^{\prime}$. We must compute

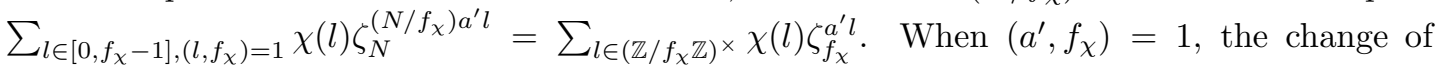
variables $l^{\prime}:=a^{\prime} l$ and the multiplicativity of $\chi$ yield the result. If $\left(a^{\prime}, f_{\chi}\right) \neq 1$, let $f^{\prime}$ be the order of $\zeta_{f_{\chi}}^{a^{\prime}} ; f^{\prime}$ divides strictly $f_{\chi}$. The last sum is rewritten as follows

$$
\sum_{l^{\prime} \in\left(\mathbb{Z} / f^{\prime} \mathbb{Z}\right)^{\times}}\left(\zeta_{f_{\chi}}^{a^{\prime}}\right)^{l^{\prime}}\left(\sum_{l \in\left(\mathbb{Z} / f_{\chi} \mathbb{Z}\right)^{\times}, l \equiv l^{\prime}\left(f_{\chi}\right)} \chi(l)\right) .
$$

Since the restriction of $\chi$ to the kernel of $\left(\mathbb{Z} / f_{\chi} \mathbb{Z}\right)^{\times} \rightarrow\left(\mathbb{Z} / f^{\prime} \mathbb{Z}\right)^{\times}$is nontrivial, we get

$$
\sum_{l \in\left(\mathbb{Z} / f_{\chi} \mathbb{Z}\right)^{\times}, l \equiv 1\left(f_{\chi}\right)} \chi(l)=0,
$$

and since $\left\{l \in\left(\mathbb{Z} / f_{\chi} \mathbb{Z}\right)^{\times}, l \equiv l^{\prime}\left(f_{\chi}\right)\right\}$ is a coset space under this kernel, $\sum_{l \in\left(\mathbb{Z} / f_{\chi} \mathbb{Z}\right)^{\times}, l \equiv l^{\prime}\left(f_{\chi}\right)} \chi(l)=$ 0 . So $\sum_{l \in\left[0, f_{\chi}-1\right],\left(l, f_{\chi}\right)=1} \chi(l) \zeta_{N}^{\left(N / f_{\chi}\right) a^{\prime} l}=0$.

Now $(60)$ is equal to $2\left(-N / f_{\chi}\right)^{1-n} \tau(\chi) L\left(n, \chi^{-1}\right)$, where $L(s, \chi)$ is the Dirichlet $L$-function $\sum_{k \geq 1,\left(k, f_{\chi}\right)=1} \chi(k) / k^{s}$.

The Euler expansion of $L(s, \chi)$ implies that when $n>1, L(n, \chi) \neq 0$ for any $\chi$. According to [W], Lemma 4.8, the Gauss sum $\tau(\chi)$ is also nonzero. It follows that $v_{\chi} \neq 0$.

Let us assume now that $n=1$ and $\chi$ is even. The coefficient of $[\zeta]$ in $v_{\chi}$ is equal to

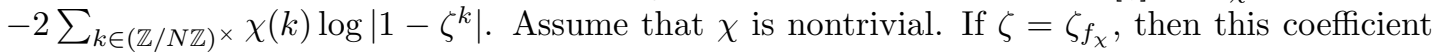
is equal to

$$
-2 \frac{\phi(N)}{\phi\left(f_{\chi}\right)} \sum_{k \in\left(\mathbb{Z} / f_{\chi} \mathbb{Z}\right)^{\times}} \chi(k) \log \left|1-\zeta_{f_{\chi}}^{k}\right|,
$$

where $\phi$ is the Euler function. According to [W], Theorem 4.9, this is equal to $2 \frac{\phi(N)}{\phi\left(f_{\chi}\right)} \frac{f_{\chi}}{\tau\left(\chi^{-1}\right)} L\left(1, \chi^{-1}\right)$ (which can be rewritten $2 \frac{\phi(N)}{\phi\left(f_{\chi}\right)} \tau(\chi) L\left(1, \chi^{-1}\right)$ according to [W], Lemma 4.8), and according to [W], Corollary 4.4, $L\left(1, \chi^{-1}\right) \neq 0$ if $\chi \neq 1$, hence $v_{\chi}$ is nonzero. 
Assume now that $n=1$ and $\chi=1$. Let $p$ be a prime divisor of $N$. The coefficient of $\left[\zeta_{p}\right]$ in $v_{1}$ is equal to

$$
-2 \frac{\phi(N)}{\phi(p)} \sum_{k \in(\mathbb{Z} / p \mathbb{Z})^{\times}} \log \left|1-\zeta_{p}^{k}\right|=-2 \frac{\phi(N)}{\phi(p)} \log (p),
$$

hence $v_{1}$ is also $\neq 0$.

So

Theorem 11.6. Let $N \geq 2$ and $\nu$ be the number of distinct prime factors of $N$. Then:

- $\mathfrak{g r t m}_{(\overline{1}, 1)}(N, \mathbb{C})_{1}^{+, \mathrm{ab}}=\nu[1]+\sum_{\chi}$ even character of $(\mathbb{Z} / N \mathbb{Z})^{\times}[\chi]$ as $(\mathbb{Z} / N \mathbb{Z})^{\times}$-modules. The map $\mathfrak{g r t m}_{(\overline{1}, 1)}(N, \mathbb{C})_{1}^{+} \rightarrow \operatorname{Hom}(\operatorname{Div}(N), \mathbb{C})$ is surjective; it is a bijection when restricted to the $(\mathbb{Z} / N \mathbb{Z})^{\times}$-invariant part of the source of this map.

- If $n \geq 3$ is odd, then $\mathfrak{g r t m}_{(\overline{1}, 1)}(N, \mathbb{C})_{n}^{+, \text {ab }} \geq \sum_{\chi \text { even character }}[\chi]$ as $(\mathbb{Z} / N \mathbb{Z})^{\times}$-modules.

$$
\text { of }(\mathbb{Z} / N \mathbb{Z})^{\times}
$$

- if $n \geq 2$ is even, then $\mathfrak{g r t m}_{(\overline{1}, 1)}(N, \mathbb{C})_{n}^{+, \mathrm{ab}} \geq \sum_{\chi}$ odd character $[\chi]$ as $(\mathbb{Z} / N \mathbb{Z})^{\times}$-modules. of $(\mathbb{Z} / N \mathbb{Z})^{\times}$

In particular, set $d(n):=\operatorname{dim}_{\mathbb{Q}} \mathfrak{g r t m}_{(\overline{1}, 1)}(N, \mathbb{Q})_{n}^{+, \text {ab }}, D(t)=\sum_{n \geq 1} d(n) t^{n}$. Then:

- If $N=2, D(t) \geq t /\left(1-t^{2}\right)$;

- if $N \geq 3, D(t) \geq \frac{\phi(N)}{2} \frac{t}{1-t}+(\nu-1) t$.

Note that $D(t)$ coincides with $f(t)$ from [DG], proof of Cor. 5.25.

Proof. Proposition 11.4 implies the two last statements. Let us compute the character of $\mathfrak{g r t m}_{(\overline{1}, 1)}(N, \mathbb{C})_{1}^{+, \text {ab }}$. Recall that $\mathfrak{g r t m}_{(\overline{1}, 1)}(N, \mathbb{Q})_{1}^{+, \text {ab }}$ is the space of pairs

$$
\left(\sum_{a \in \mathbb{Z} / N \mathbb{Z}} x(a) b(a), \rho\right)
$$

where $\rho \in \mathbb{Q}^{\nu}=\operatorname{Hom}(\operatorname{Div}(N), \mathbb{Q})$ and $x(a) \in \mathbb{Q}$, such that:

a) $x(0)=0$,

b) for any $a \in \mathbb{Z} / N \mathbb{Z}, x(a)=x(-a)$, and

c) if $N=d N^{\prime}$, then for any $a^{\prime} \in \mathbb{Z} / N^{\prime} \mathbb{Z}-\{0\}$, we have $x\left(d a^{\prime}\right)=\sum_{k=0}^{d-1} x\left(a^{\prime}+k N^{\prime}\right)$, and

d) if $N=d N^{\prime}$, then $\sum_{k=0}^{d-1} x\left(k N^{\prime}\right)=\rho(d)$.

As in Lemma 10.2, one shows that if $(x(a))_{a \in \mathbb{Z} / N \mathbb{Z}}$ satisfies a), b), c), then $d \mapsto \rho(d)$ defined

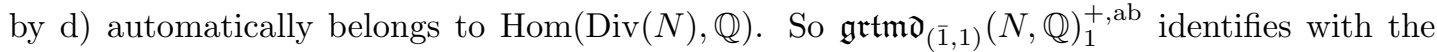

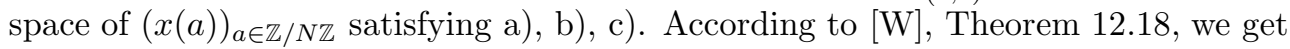

$$
\operatorname{dim}_{\mathbb{Q}} \mathfrak{g r t m}_{(\overline{1}, 1)}(N, \mathbb{Q})_{1}^{+, \mathrm{ab}}=\frac{\phi(N)}{2}+\nu-1 .
$$

The multiplicity of an odd $\chi$ in $\mathfrak{g r t m}_{(\overline{1}, 1)}(N, \mathbb{C})_{1}^{+, \text {ab }}$ is 0 , and the multiplicity of an even $\chi \neq 1$ is $\geq 1$.

Let us compute the multiplicity of the trivial character $\chi=1$, i.e., $\operatorname{dim}_{\mathbb{Q}}\left(\mathfrak{g r t m}_{(\overline{1}, 1)}(N, \mathbb{Q})_{1}^{+, \mathrm{ab}}\right)^{(\mathbb{Z} / N \mathbb{Z})^{\times}}$. We have a morphism $\left(\mathfrak{g r t m} \mathfrak{d}_{(\overline{1}, 1)}(N, \mathbb{Q})_{1}^{+, \text {ab }}\right)^{*} \rightarrow \mathbb{R}$, taking $x(a)$ to $\log \left|1-\zeta_{N}^{a}\right|$. The image of the invariant part of $\left(\mathfrak{g r t m}_{(\overline{1}, 1)}(N, \mathbb{Q})_{1}^{+, \text {ab }}\right)^{*}$ is the $\mathbb{Q}$-linear span of all $f(\zeta)=\sum_{k \in(\mathbb{Z} / N \mathbb{Z})^{\times}} \log \mid 1-$ $\zeta^{k} \mid$, where $\zeta \in \mu_{N}(\mathbb{C})$. Now either the order of $\zeta$ is a prime power $p^{a}$ dividing $N$, and $f(\zeta)=\frac{\phi(N)}{\phi\left(p^{a}\right)} \log (p)$; or the order of $\zeta$ is not a prime power, and $f(\zeta)=0$. Hence the invariant part of $\left(\mathfrak{g r t m}_{(\overline{1}, 1)}(N, \mathbb{Q})_{1}^{+, \text {ab }}\right)^{*}$ maps surjectively to $\operatorname{Span}_{\mathbb{Q}}\{\log (p), p$ prime dividing $N\}$. By uniqueness of the prime powers decomposition, the $\log (p)$ form a free family over $\mathbb{Q}$, hence $\operatorname{dim}_{\mathbb{Q}} \operatorname{Span}_{\mathbb{Q}}\{\log (p), p$ prime dividing $N\}=\nu$. So $\operatorname{dim}_{\mathbb{Q}}\left(\mathfrak{g r t m}_{(\overline{1}, 1)}(N, \mathbb{Q})_{1}^{+, \text {ab }}\right)^{(\mathbb{Z} / N \mathbb{Z})^{\times}} \geq \nu$. 
Let us now show that $\left(\mathfrak{g r t m}_{(\overline{1}, 1)}(N, \mathbb{Q})_{1}^{+, \mathrm{ab}}\right)^{(\mathbb{Z} / N \mathbb{Z})^{\times}} \rightarrow \operatorname{Hom}(\operatorname{Div}(N), \mathbb{Q})$ is bijective. According to what we have seen, it suffices to show that the composed map $\operatorname{Hom}(\operatorname{Div}(N), \mathbb{Q})^{*} \rightarrow$ $\left(\mathfrak{g r t m}_{(\overline{1}, 1)}(N, \mathbb{Q})_{1}^{+}\right)^{*} \rightarrow \operatorname{Span}_{\mathbb{Q}}\{\log (p), p$ prime dividing $N\}$ is bijective. The first map takes $p$ to the linear map $\left(\sum_{a} x(a) b(a), \rho\right) \mapsto \rho(p)$, which can be identified in view of $\left.\mathrm{d}\right)$ with the linear map $\sum_{a} x(a) b(a) \mapsto \sum_{k=0}^{p-1} x(k N / p)$. The image of this element by the second map is then $\sum_{k=0}^{p-1} \log \left|1-\zeta_{N}^{k N / p}\right|=\log (p)$. So the composed map is $\operatorname{Hom}(\operatorname{Div}(N), \mathbb{Q})^{*} \rightarrow \operatorname{Span}_{\mathbb{Q}}\{\log (p), p$ prime dividing $N\}, p \mapsto \log (p)$, which is bijective, as wanted.

Comparing (61) with the lower bounds obtained for the multiplicities, we obtained the wanted formula for the character of $\mathfrak{g r t m} \mathfrak{d}_{(\overline{1}, 1)}(N, \mathbb{C})_{1}^{+, \mathrm{ab}}$.

\section{2. $\mathfrak{g r t m}_{(\overline{1}, 1)}(N, \mathbf{k}), \mathfrak{g r t m}_{(\overline{1}, 1)}(N, \mathbf{k})$ AND SPECIAL DERIVATIONS}

In this section, we relate $\mathfrak{g r t m}_{(\overline{1}, 1)}(N, \mathbf{k})$ and $\mathfrak{g r t m} \mathfrak{d}_{(\overline{1}, 1)}(N, \mathbf{k})$ with analogues of Ihara's algebra and Drinfeld's Hamiltonian interpretation (see [Dr2], Section 6).

Recall that $\mathfrak{f}_{N+1}=\mathfrak{f}(A, b(a), a \in \mathbb{Z} / N \mathbb{Z})$ and $C$ is given by $A+C+\sum_{a \in \mathbb{Z} / N \mathbb{Z}} b(a)=0$.

The dihedral group $D_{N}:=(\mathbb{Z} / N \mathbb{Z}) \rtimes(\mathbb{Z} / 2 \mathbb{Z})$ acts on $\mathfrak{f}_{N+1}$ as follows:

- the element $a^{\prime} \in \mathbb{Z} / N \mathbb{Z}$ acts by the automorphism $\theta_{a^{\prime}}: A \mapsto A, b(a) \mapsto b\left(a+a^{\prime}\right)$;

- the element $\overline{1} \in \mathbb{Z} / 2 \mathbb{Z}$ acts by the involutive automorphism $\theta: A \mapsto C, b(a) \mapsto b(-a)$.

As in [Ih1, Dr2], let us say that derivation $\partial$ of $\mathfrak{f}_{N+1}$ is special iff there exist $R_{A}, R_{C}, R_{a} \in$ $\mathfrak{f}_{N+1}$, such that $\partial(A)=\left[R_{A}, A\right], \partial(C)=\left[R_{C}, C\right], \partial(b(a))=\left[R_{a}, b(a)\right]$. The special derivations form a Lie algebra $\operatorname{SDer}\left(\mathfrak{f}_{N+1}\right)$. The inner derivations of $\mathfrak{f}_{N+1}$ form a Lie ideal $\operatorname{Inn}\left(\mathfrak{f}_{N+1}\right) \subset$ $\operatorname{SDer}\left(\mathfrak{f}_{N+1}\right)$. The group $D_{N}$ acts by automorphisms of $\operatorname{SDer}\left(\mathfrak{f}_{N+1}\right)$ and preserves $\operatorname{Inn}\left(\mathfrak{f}_{N+1}\right)$; so does $(\mathbb{Z} / N \mathbb{Z})^{\times}$.

Let us denote by $\operatorname{Ih}(N, \mathbf{k})$ the set of $\psi \in \mathfrak{t}_{3, N}^{0}=\mathfrak{f}_{N+1}$, satisfying relations (38), (39) (but not necessarily (40)). This is a Lie algebra when equipped with the bracket (43), (45). The elements $A, b(0)$ are central in $\operatorname{Ih}(N, \mathbf{k}) ; \operatorname{Ih}(N, \mathbf{k})$ is graded, we set $\operatorname{Ih}^{+}(N, \mathbf{k})_{1}:=\operatorname{Ih}^{+}(N, \mathbf{k}) \cap$ $\operatorname{Span}_{\mathbf{k}}(b(a), a \neq 0), \operatorname{Ih}^{+}(N, \mathbf{k}):=\operatorname{Ih}^{+}(N, \mathbf{k})_{1} \oplus \operatorname{Ih}(N, \mathbf{k})_{2} \oplus \cdots$. So $\operatorname{Ih}(N, \mathbf{k}):=\operatorname{Ih}^{+}(N, \mathbf{k}) \oplus$ $(\mathbf{k} A \oplus \mathbf{k} b(0))$, where the first summand in a subalgebra and the second summand is central.

Proposition 12.1. We have an isomorphism of graded Lie algebras with $(\mathbb{Z} / N \mathbb{Z})^{\times}$-action

$$
\operatorname{Ih}^{+}(N, \mathbf{k}) \simeq\left(\operatorname{SDer}\left(\mathfrak{f}_{N+1}\right) / \operatorname{Inn}\left(\mathfrak{f}_{N+1}\right)\right)^{D_{N}},
$$

taking $\psi$ to the class of the special derivation $\partial_{\psi}: A \mapsto[\psi(A \mid b(0), \ldots, b(N-1))-\psi(C \mid b(0), \ldots, b(1-$ $N)), A], b(a) \mapsto[\psi(A \mid b(a), \ldots, b(a+N-1)), b(a)]$.

Proof. SDer $\left(\mathfrak{f}_{N+1}\right) / \operatorname{Inn}\left(\mathfrak{f}_{N+1}\right)$ identifies with the quotient special derivations $\partial$ such that $\left.R_{C}=0\right\} / \mathbf{k} \operatorname{ad}(C)$. The statement is carried out directly in degree 1 . If now $\partial$ is such a special derivation of degree $>1$ (meaning that $R_{A}, R_{a}$ have degree $d>1$ ), then its class is $(\mathbb{Z} / N \mathbb{Z})$-invariant iff $R_{A}$ is $(\mathbb{Z} / N \mathbb{Z})$-invariant and $R_{a}=\theta_{a}\left(R_{0}\right)$. So $R_{0}, R_{A}$ are such that

$$
\left[R_{A}, A\right]+\sum_{a \in \mathbb{Z} / N \mathbb{Z}}\left[\theta_{a}\left(R_{0}\right), b(a)\right]=0
$$

and $\partial$ is given by $A \mapsto\left[R_{A}, A\right], b(a) \mapsto\left[\theta_{a}\left(R_{0}\right), b(a)\right]$. Now the special derivation representing $\theta \circ \partial \circ \theta^{-1}$ is $A \mapsto\left[-\theta\left(R_{A}\right), A\right], b(a) \mapsto\left[\theta \theta_{-a}\left(R_{0}\right)-\theta\left(R_{A}\right), b(a)\right]$. We have $\partial=\theta \circ \partial \circ \theta^{-1}$, hence $R_{A}+\theta\left(R_{A}\right)=0$; and $R_{A}=\theta_{-a}\left(R_{0}\right)-\theta \theta_{a}\left(R_{0}\right)=\theta_{-a}(\mathrm{id}-\theta)\left(R_{0}\right)$ for any $a$. Hence $R_{A}=(\mathrm{id}-\theta)\left(R_{0}\right)$, so $\partial=\partial_{R_{0}} ;$ and $R_{A}$ is $(\mathbb{Z} / N \mathbb{Z})$-invariant, which means that $R_{0}$ satisfies (38); finally, (62) implies that $R_{0}$ satisfies (39). Hence we have an isomorphism of graded vector spaces. The proof that it is a Lie morphism is as in [Dr2].

The Hamiltonian interpretation of $\operatorname{Ih}(\mathbf{k})$ from [Dr2] can be generalized as follows. We first recall some notation. Let $\mathcal{F}\left(\mathfrak{f}_{N+1}\right)$ be the quotient of $\mathfrak{f}_{N+1}^{\otimes 2}$ by the subspace generated by the 
elements $x \otimes y-y \otimes x,[x, y] \otimes z-x \otimes[y, z], x, y, z \in \mathfrak{f}_{N+1}$. As in [Dr2], any $f \in \mathcal{F}\left(\mathfrak{f}_{N+1}\right)$ yields a universal function $f_{\mathfrak{g}}:\left(\mathfrak{g}^{*}\right)^{N+1} \rightarrow \mathbf{k}$, where $\mathfrak{g}$ is a finite dimensional Lie algebra with nondegenerate invariant scalar product. Using the Kostant-Kirillov Poisson bracket on $\left(\mathfrak{g}^{*}\right)^{n}$, this interpretation allows to define a Lie bracket on $\mathcal{F}\left(\mathfrak{f}_{N+1}\right)$.

Proposition 12.2. 1) $\mathcal{F}\left(\mathfrak{f}_{N+1}\right)^{D_{N}} \subset \mathcal{F}\left(\mathfrak{f}_{N+1}\right)$ is a Lie subalgebra.

2) There is an isomorphism of graded Lie algebras with $(\mathbb{Z} / N \mathbb{Z})^{\times}$-action $\operatorname{Ih}(N, \mathbf{k}) \simeq \mathcal{F}\left(\mathfrak{f}_{N+1}\right)^{D_{N}}$.

Proof. Similar to [Dr2]; the map $\psi \mapsto f$ is given by the formulas

$$
\frac{\partial f}{\partial A}=(\mathrm{id}-\theta)(\psi), \frac{\partial f}{\partial b(a)}=\theta(a)(\psi) ;
$$

the fact that $f$ is $D_{N}$-invariant follows from the properties of $\psi$ and the identities $(\partial / \partial A) \circ \theta_{1}=$ $\theta_{1} \circ(\partial / \partial A),(\partial / \partial b(a)) \circ \theta_{1}=\theta_{1} \circ(\partial / \partial b(a-1))$, and $(\partial / \partial A) \circ \theta=-\theta \circ(\partial / \partial A),(\partial / \partial b(a)) \circ \theta=$ $\theta \circ(\partial / \partial b(-a))$.

In particular, the element $b(0) \in \operatorname{Ih}(N, \mathbf{k})$ corresponds to $\frac{1}{2} \sum_{a \in \mathbb{Z} / N \mathbb{Z}}(b(a), b(a))$, and $A$ corresponds to $-(A, C)$.

We also define a Lie algebra Ihdist $(N, \mathbf{k})$ by forgetting the pentagon conditions in the definition of $\mathfrak{g r t m} \mathfrak{d}_{(\overline{1}, 1)}(M, \mathbf{k})$.

If $N \mid N^{\prime}$, we have Lie algebra morphisms $\pi_{N N^{\prime}}, \delta_{N_{N^{\prime}}}: \mathcal{F}\left(\mathfrak{f}_{N+1}\right)^{D_{N}} \rightarrow \mathcal{F}\left(\mathfrak{f}_{N^{\prime}+1}\right)^{D_{N^{\prime}}}$, respectively obtained by replacing $b(a)$ by $b^{\prime}\left(a \bmod N^{\prime}\right)$ in a given expression, or by replacing it by $b^{\prime}(a / d)$ if $d \mid a$, and by 0 otherwise.

Then we define a Lie algebra $\mathcal{F}(N, \mathbf{k}) \subset \mathcal{F}\left(\mathfrak{f}_{N+1}\right)^{D_{N}} \times \mathbf{k}^{\nu}$ as the set of pairs $(f, \rho)$ such that for any $N^{\prime} \mid N, \pi_{N N^{\prime}}(f)=\delta_{N N^{\prime}}(f)+\frac{\rho(d)}{2} \sum_{a \in \mathbb{Z} / N \mathbb{Z}}(b(a), b(a))$.

Proposition 12.3. $\operatorname{Ih}(N, \mathbf{k}) \simeq \mathcal{F}\left(\mathfrak{f}_{N+1}\right)^{D_{N}}$ restricts to an isomorphism of graded Lie algebras with $(\mathbb{Z} / N \mathbb{Z})^{\times}$-action $\operatorname{Ihdist}(N, \mathbf{k}) \simeq \mathcal{F}(N, \mathbf{k})$.

Proof. Straightforward.

\section{Actions of $\operatorname{Gal}(\overline{\mathbb{Q}} / \mathbb{Q})$}

In this section, we show that the natural action $([\mathrm{Gr}])$ of $G_{\mathbb{Q}}:=\operatorname{Gal}(\overline{\mathbb{Q}} / \mathbb{Q})$ on the orbifold fundamental group ${ }^{7}$ of $\left(\mathbb{C}^{\times}-\mu_{N}(\mathbb{C})\right) / D_{N}$ factors through $\widehat{\mathrm{GT}}$ in the profinite setup, and through $\operatorname{GTMD}(N)_{l}$ in the pro-l setup. Here $\mathbb{Z} / N \mathbb{Z} \subset D_{N}$ acts by multiplication (using the isomorphism $\mathbb{Z} / N \mathbb{Z} \simeq \mu_{N}(\mathbb{C})$ induced by $\left.\zeta_{N}\right)$ and $\overline{1} \in \mathbb{Z} / 2 \mathbb{Z}$ acts by $z \mapsto 1 / z$.

We set $M_{N}:=\mathbb{C}^{\times}-\mu_{N}(\mathbb{C}), X_{N}:=M_{N} / D_{N}$. We first describe the exact sequence $1 \rightarrow$ $\pi_{1}\left(M_{N}\right) \simeq F_{N+1} \rightarrow \pi_{1}^{\text {orb }}\left(X_{N}\right) \rightarrow D_{N} \rightarrow 1$ (the base-point $x_{0}$ is close to 0 and $\left.>0\right)$. Recall that $B_{2}^{1}=\langle\tau, \sigma\rangle /\left((\sigma \tau)^{2}=(\tau \sigma)^{2}\right)$; the center of $B_{2}^{1}$ is $Z\left(B_{2}^{1}\right) \simeq \mathbb{Z}=\left\langle(\tau \sigma)^{2}\right\rangle$. We have an exact seqence $1 \rightarrow K_{2, N} \rightarrow B_{2}^{1} \rightarrow(\mathbb{Z} / N \mathbb{Z})^{2} \rtimes \mathfrak{S}_{2} \rightarrow 1$, where the second map is $\tau \mapsto(\overline{1}, \overline{0}) \in$ $(\mathbb{Z} / N \mathbb{Z})^{2} \subset(\mathbb{Z} / N \mathbb{Z})^{2} \rtimes \mathfrak{S}_{2}$ and $\sigma \mapsto(21) \in \mathfrak{S}_{2} \subset(\mathbb{Z} / N \mathbb{Z})^{2} \rtimes \mathfrak{S}_{2}$; the kernel is presented by $K_{2, N}=\left\langle X_{01}, X_{02}, x_{12}(0), \ldots, x_{12}(N-1)\right\rangle /\left(X_{02} x_{12}(0) \ldots x_{12}(N-1) X_{01}\right.$ is central $)$, where $X_{01}=\tau^{N}, x_{12}(\alpha)=\tau^{\alpha} \sigma^{2} \tau^{-\alpha}$, and $X_{02}=\left(\sigma \tau \sigma^{-1}\right)^{N}$. Then $Z\left(B_{2}^{1}\right) \cap K_{2, N}$ is generated by $X_{02} x_{12}(0) \ldots x_{12}(N-1) X_{01}=(\tau \sigma)^{2 N}$. The image of $(\tau \sigma)^{2}$ in $(\mathbb{Z} / N \mathbb{Z})^{2} \rtimes \mathfrak{S}_{2}$ is $(\overline{1}, \overline{1}) \in(\mathbb{Z} / N \mathbb{Z})^{2}$, which generates a central $\mathbb{Z} / N \mathbb{Z}$. Factoring the above exact sequence by $0 \rightarrow Z\left(B_{2}^{1}\right) \cap K_{2, N} \rightarrow$ $Z\left(B_{2}^{1}\right) \rightarrow \mathbb{Z} / N \mathbb{Z} \rightarrow 0$ (which coincides with $0 \rightarrow \mathbb{Z} \stackrel{N \times-}{\rightarrow} \mathbb{Z} \rightarrow \mathbb{Z} / N \mathbb{Z} \rightarrow 0$ ), we get an exact

\footnotetext{
${ }^{7}$ If the group $\Gamma$ acts on the topological space $X$, and $x_{0} \in X$, then $\pi_{1}^{o r b}\left(X / \Gamma,\left[x_{0}\right]\right)$ is the set of pairs $(\gamma, \ell)$, where $\gamma \in \Gamma$ and $\ell$ is the isotopy class of a path from $x_{0}$ to $\gamma x_{0}$; the composition is $(\gamma, \ell)\left(\gamma^{\prime}, \ell^{\prime}\right)=\left(\gamma \gamma^{\prime}, \gamma \ell^{\prime} \circ \ell\right)$. We have an exact sequence $1 \rightarrow \pi_{1}\left(X, x_{0}\right) \rightarrow \pi_{1}^{\text {orb }}\left(X / \Gamma,\left[x_{0}\right]\right) \rightarrow \Gamma \rightarrow 1$.
} 
sequence $1 \rightarrow F_{N+1} \rightarrow B_{2}^{1} / Z\left(B_{2}^{1}\right) \rightarrow D_{N} \rightarrow 1$, where $F_{N+1}:=K_{2, N} /\left(X_{02} x_{12}(0) \ldots x_{12}(N-\right.$ 1) $\left.X_{01}=1\right)$ is freely generated by $X_{01}, x_{12}(0), \ldots, x_{12}(N-1)$. We then have

$$
\pi_{1}^{\text {orb }}\left(X_{N},\left[x_{0}\right]\right) \simeq B_{2}^{1} / Z\left(B_{2}^{1}\right) \simeq\langle\tau, \sigma\rangle /\left((\tau \sigma)^{2}=1\right) ;
$$

the projection $\pi_{1}^{\text {orb }}\left(X_{N},\left[x_{0}\right]\right) \stackrel{\tilde{\varphi}_{N}}{\rightarrow} D_{N}=(\mathbb{Z} / N \mathbb{Z}) \rtimes(\mathbb{Z} / 2 \mathbb{Z})$ coincides with the above map, so is given by $\tau \mapsto(\overline{1}, \overline{0}), \sigma \mapsto(\overline{0}, \overline{0})$; this is the factorization by the relations $\tau^{N}=\sigma^{2}=1$.

The isomorphism (63) is described geometrically by $\tau \mapsto$ the counterclockwise path from $x_{0}$ to $\zeta_{N} x_{0}$, and $\sigma \mapsto$ the path from $x_{0}$ to $1 / x_{0}$, contained in $\{z \mid 0 \leq \arg (z) \leq 2 \pi / N\}-\left\{0,1, \zeta_{N}\right\}$. The prop- $l$ (resp., algebraic) fundamental group of $X_{N}$ is $\pi_{1}^{\text {orb }}\left(X_{N}\right)\left(\tilde{\varphi}_{N}, l\right)$ (resp., $\left.\pi_{1}^{\text {orb }}\left(X_{N}\right)^{\wedge}\right)$; this is $\operatorname{Gal}\left(F_{l} / E\right)$, where $E=\overline{\mathbb{Q}}(z)^{D_{N}}, F_{l}$ is the maximal pro-l algebraic extension of $E$ in $\overline{\mathbb{Q}}\left(z^{1 / n}, n>0\right)$, unramified outside $0, \infty, \mu_{N}(\overline{\mathbb{Q}})$ (resp., $\operatorname{Gal}(F / E)$, where the pro-l condition is dropped in the definition of $F$ ).

The action of $G_{\mathbb{Q}}$ on $\pi_{1}^{\text {orb }}\left(X_{N}\right)\left(\tilde{\varphi}_{N}, l\right)$ factors through $\operatorname{GTMD}(N)_{l}$, and the action of the latter group takes $(\lambda, \mu, f, g)$ to the automorphism $\tau \mapsto \tau^{\mu}, \sigma \rightarrow g^{-1}\left(\tau, \sigma^{2}\right) \sigma^{\lambda} g\left(\tau, \sigma^{2}\right)$ (one can prove that the octogon identity is equivalent to the condition that this is an automorphism of $\left.\pi_{1}^{\text {orb }}\left(X_{N}\right)\left(\tilde{\varphi}_{N}, l\right)\right)$. In the same way, the action of $G_{\mathbb{Q}}$ on $\pi_{1}^{\text {orb }}\left(X_{N}\right)^{\wedge}$ factors through $\widehat{\mathrm{GT}}$ and it given by the same formulas.

When $N=1,2,4$, the subgroup $\Gamma_{N}$ of $\mathrm{PSL}_{2}(\mathbb{C})$ of automorphisms of $\mathbb{C}^{\times}-\mu_{N}(\mathbb{C})$ is strictly larger than $D_{N}$. Taking into account these additional symmetries, one defines subgroups $\operatorname{GTMD}^{\prime}(N)_{l} \subset \operatorname{GTMD}(N)_{l}$, such that we have morphisms $G_{\mathbb{Q}} \hookrightarrow \widehat{\mathrm{GT}} \rightarrow \operatorname{GTMD}^{\prime}(N)_{l} \rightarrow$ $\operatorname{Aut}\left(\pi_{1}^{\text {orb }}\left(\mathbb{C}^{\times}-\mu_{N}(\mathbb{C}) / \Gamma_{N}\right)^{(l)}\right)$. We will return to this question elsewhere.

For completeness, we recall the action of $G_{\mathbb{Q}}$ on $\pi_{1}^{\text {orb }}\left(\mathbb{C}^{\times}-\{1\} / \mathfrak{S}_{3}\right)^{(l)}\left(\right.$ when $N=1, \Gamma_{N}=$ $\left.\mathfrak{S}_{3}\right)$, described in [Dr2], and show its compatibility with its action on $\pi_{1}^{\text {orb }}\left(\mathbb{C}^{\times}-\{1\} / D_{1}\right)^{(l)}$ described above. The exact sequence $1 \rightarrow F_{2} \rightarrow \pi_{1}^{\text {orb }}\left(\mathbb{C}^{\times}-\{1\} / \mathfrak{S}_{3},\left[x_{0}\right]\right) \rightarrow \mathfrak{S}_{3} \rightarrow 1$ coincides with $1 \rightarrow F_{2} \rightarrow B_{3} / Z\left(B_{3}\right) \rightarrow \mathfrak{S}_{3} \rightarrow 1$, where $B_{3}=\left\langle\sigma_{1}, \sigma_{2}\right\rangle /\left(\sigma_{1} \sigma_{2} \sigma_{1}=\sigma_{2} \sigma_{1} \sigma_{2}\right) ; Z\left(B_{3}\right)=$ $\left\langle\left(\sigma_{1}^{2} \sigma_{2}\right)^{2}\right\rangle \simeq \mathbb{Z}$, and the morphism $B_{3} / Z\left(B_{3}\right) \rightarrow \mathfrak{S}_{3}$ is $\bar{\sigma}_{1} \mapsto(12), \bar{\sigma}_{2} \mapsto(23)$; it coincides with the factorization by the relations $\bar{\sigma}_{1}^{2}=\bar{\sigma}_{2}^{2}=1$. The identification $\pi_{1}^{\text {orb }}\left(\mathbb{C}^{\times}-\{1\} / \mathfrak{S}_{3}\right) \simeq$ $B_{3} / Z\left(B_{3}\right)$ takes $\bar{\sigma}_{1}$ to the path $x_{0} \mapsto 1-x_{0}$ contained in $] 0,1\left[\right.$, and $\bar{\sigma}_{2}$ to the path $x_{0} \mapsto 1 / x_{0}$ contained in $\{z \mid \Im(z) \geq 0\}-\{0,1\}$.

We then have an inclusion $\pi_{1}^{\text {orb }}\left(\mathbb{C}^{\times}-\{1\} / D_{1},\left[x_{0}\right]\right) \subset \pi_{1}^{\text {orb }}\left(\mathbb{C}^{\times}-\{1\} / \mathfrak{S}_{3},\left[x_{0}\right]\right)$, corresponding to $B_{2}^{1} / Z\left(B_{2}^{1}\right) \rightarrow B_{3} / Z\left(B_{3}\right), \bar{\tau} \mapsto \bar{\sigma}_{1}^{2}, \bar{\sigma} \mapsto \bar{\sigma}_{1}$. The action of $\mathrm{GT}_{l}$ on $\pi_{1}\left(\mathbb{C}^{\times}-\{1\} / \mathfrak{S}_{3}\right)^{(l)}$ takes $(\lambda, f)$ to $\bar{\sigma}_{1} \mapsto \bar{\sigma}_{1}^{\lambda}, \bar{\sigma}_{1} \bar{\sigma}_{2} \bar{\sigma}_{1} \mapsto \bar{\sigma}_{1} \bar{\sigma}_{2} \bar{\sigma}_{1} f\left(\bar{\sigma}_{1}^{2}, \bar{\sigma}_{2}^{2}\right)$; this restricts to the action of $\mathrm{GT}_{l}$ on $\pi_{1}\left(\mathbb{C}^{\times}-\{1\} / D_{1}\right)^{(l)}$ as $\bar{\sigma}_{1} \bar{\sigma}_{2} \bar{\sigma}_{1} f\left(\bar{\sigma}_{1}^{2}, \bar{\sigma}_{2}^{2}\right)=\bar{\sigma}_{1}^{\lambda} f^{-1}\left(\bar{\sigma}_{1}^{2}, \bar{\sigma}_{2}^{2}\right) \bar{\sigma}_{2}^{\lambda} f\left(\bar{\sigma}_{1}^{2}, \bar{\sigma}_{2}^{2}\right) f\left(\bar{\sigma}_{1}^{2}, \bar{\sigma}_{2}^{2}\right) \bar{\sigma}_{1}^{\lambda}$ (which is used implicitly in [Dr2] and follows from the hexagon identity).

\section{Appendix A. The coefficients of $\Psi_{\mathrm{KZ}}$}

Let $A, b[\zeta], \zeta \in \mu_{N}(\mathbb{C})$ be free variables (here $A$ and $b[\zeta]$ have degree 1 ; the identification is $\left.A=t_{0}^{01}, b[\zeta]=t[\zeta]^{12}\right)$. Then $\Psi_{\mathrm{KZ}}$ may be identified with the renormalized holonomy from 0 to 1 of

$$
\frac{\mathrm{d}}{\mathrm{d} z} H(z)=\left(\frac{A}{z}+\sum_{\zeta \in \mu_{N}(\mathbb{C})} \frac{b[\zeta]}{z-\zeta}\right) H(z)
$$

i.e. $\Psi_{\mathrm{KZ}}=H_{1}^{-1} H_{0}$, where $H_{0}(z) \sim z^{A}$ and $H_{1}(z) \sim(1-z)^{b[1]}$.

Le and Murakami's formula ([LM]) for the KZ associator can be generalized to $\Psi_{\mathrm{KZ}}$, showing that $\Psi_{\mathrm{KZ}}$ may be viewed as a generating series for multiple polylogarithms (MPL's) at $N$ th roots of unity. 
We first define these numbers. If $\zeta_{1}, \ldots, \zeta_{r} \in \mu_{N}(\mathbb{C})$, and $s_{1}, \ldots, s_{r}$ are positive integers such that $\left(s_{r}, \zeta_{r}\right) \neq(1,1)$, we set

$$
\operatorname{Li}_{s_{1}, \ldots, s_{r}}\left(\zeta_{1}, \ldots, \zeta_{r}\right)=\sum_{1 \leq n_{1}<\ldots<n_{r}} \frac{\zeta_{1}^{n_{1}} \cdots \zeta_{r}^{n_{r}}}{n_{1}^{s_{1}} \cdots n_{r}^{s_{r}}}
$$

It is well-known that these numbers correspond bijectively to iterated integrals. Set $\tilde{\mu}_{N}=$ $\{0\} \cup \mu_{N}(\mathbb{C})$. If $\left(\tilde{\zeta}_{1}, \ldots, \tilde{\zeta}_{m}\right)$ is a sequence with values in $\tilde{\mu}_{N}$, such that $\tilde{\zeta}_{1} \neq 0$ and $\tilde{\zeta}_{m} \neq 1$, we set

$$
I\left(\tilde{\zeta}_{1}, \ldots, \tilde{\zeta}_{m}\right)=\int_{0 \leq t_{1} \leq \ldots \leq t_{r} \leq 1} \omega_{\tilde{\zeta}_{1}}\left(t_{1}\right) \wedge \cdots \wedge \omega_{\tilde{\zeta}_{r}}\left(t_{r}\right), \quad \omega_{0}=\mathrm{d} t / t, \omega_{\zeta}=\mathrm{d} t /(\zeta-t) .
$$

Then

$$
\operatorname{Li}_{s_{1}, \ldots, s_{r}}\left(\zeta_{1}, \ldots, \zeta_{r}\right)=I\left(\left(\zeta_{1} \cdots \zeta_{r}\right)^{-1}, 0^{\left(s_{1}-1\right)},\left(\zeta_{2} \cdots \zeta_{r}\right)^{-1}, 0^{\left(s_{2}-1\right)}, \ldots, \zeta_{r}^{-1}, 0^{\left(s_{r}-1\right)}\right)
$$

(here $0^{(i)}$ means 0 repeated $i$ times).

Proposition A.1. Set $b[0]:=A$. If $\left(\tilde{\zeta}_{1}, \ldots, \tilde{\zeta}_{r}\right)$ is a sequence in $\left(\tilde{\mu}_{N}\right)^{r}$, we set $w\left(\tilde{\zeta}_{1}, \ldots, \tilde{\zeta}_{r}\right):=$ $b\left[\tilde{\zeta}_{r}\right] \cdots b\left[\tilde{\zeta}_{1}\right]$. If $I \subset\left\{i \mid \tilde{\zeta}_{i}=0\right\}, J \subset\left\{i \mid \tilde{\zeta}_{i}=1\right\}$, set $w\left(\tilde{\zeta}_{1}, \ldots, \tilde{\zeta}_{r}\right)^{I, J}:=\prod_{i \in[1, r] \backslash(I \cup J)} b\left[\tilde{\zeta}_{i}\right]$ (the product is in decreasing order) and

$$
\tilde{w}\left(\tilde{\zeta}_{1}, \ldots, \tilde{\zeta}_{r}\right):=\sum_{I \subset\left\{i \mid \tilde{\zeta}_{i}=1\right\}, J \subset\left\{i \mid \tilde{\zeta}_{i}=0\right\}}(-1)^{\operatorname{card}(I)+\operatorname{card}(J)} b[1]^{\operatorname{card}(I)} w\left(\tilde{\zeta}_{1}, \ldots, \tilde{\zeta}_{r}\right)^{I, J} b[0]^{\operatorname{card}(J)} .
$$

Then

$$
\Psi_{\mathrm{KZ}}=\sum_{r \geq 0} \sum_{\left(\tilde{\zeta}_{1}, \ldots, \tilde{\zeta}_{r}\right) \in\left(\tilde{\mu}_{N}\right)^{r} \mid \tilde{\zeta}_{1} \neq 0, \tilde{\zeta}_{r} \neq 1}(-1)^{\operatorname{card}\left\{i \in[1, r] \mid \tilde{\zeta}_{i} \neq 0\right\}} I\left(\tilde{\zeta}_{1}, \ldots, \tilde{\zeta}_{r}\right) \tilde{w}\left(\tilde{\zeta}_{1}, \ldots, \tilde{\zeta}_{r}\right) .
$$

Proof. We denote $\Psi_{\mathrm{KZ}}$ by $\Psi$ in the course of this proof, which is parallel to [LM]. Let $\mathbb{C}\left\langle\left\langle b[\tilde{\zeta}], \tilde{\zeta} \in \tilde{\mu}_{N}\right\rangle\right.$ be the degree completion of the free algebra with generators $A, b[\zeta], \zeta \in \mu_{N}(\mathbb{C})$. Let $\alpha, \beta$ be additional indeterminates of degree 1 commuting with each other and with the $b[\tilde{\zeta}]$ $\left(\tilde{\zeta} \in \tilde{\mu}_{N}\right)$. Let $\mathbb{C}\langle\langle b[\tilde{\zeta}]\rangle\rangle_{0} \subset \mathbb{C}\langle\langle b[\tilde{\zeta}]\rangle\rangle$ be the complete subspace spanned by the monomials not starting with $b[1]$ and not ending with $b[0]$. We have a direct sum decomposition $\mathbb{C}\langle\langle b[\tilde{\zeta}]\rangle\rangle=$ $\mathbb{C}\langle\langle b[\tilde{\zeta}]\rangle\rangle_{0} \oplus(b[1] \mathbb{C}\langle\langle b[\tilde{\zeta}]\rangle\rangle+\mathbb{C}\langle\langle b[\tilde{\zeta}]\rangle\rangle b[0])$. We denote by $\pi: \mathbb{C}\langle\langle b[\tilde{\zeta}]\rangle\rangle \rightarrow \mathbb{C}\langle\langle b[\tilde{\zeta}]\rangle\rangle_{0}$ the corresponding projection map. We also have two maps

$$
\mathbb{C}\langle\langle b[\tilde{\zeta}]\rangle\rangle \stackrel{u}{\rightarrow} \mathbb{C}\langle\langle b[\tilde{\zeta}]\rangle\rangle[[\alpha, \beta]] \stackrel{v}{\rightarrow} \mathbb{C}\langle\langle b[\tilde{\zeta}]\rangle\rangle,
$$

defined by: $u$ is an algebra morphism, $u(b[0])=b[0]-\alpha, u(b[1])=b[1]-\beta, u(b[\zeta])=b[\zeta]$ for $\zeta \in \mu_{N}(\mathbb{C})-\{1\} ; v$ takes $w \alpha^{p} \beta^{q}$ to $b[1]^{q} w b[0]^{p}$, where $w \in \mathbb{C}\langle\langle b[\tilde{c}]\rangle\rangle$.

Then $v \circ u(b[1] \mathbb{C}\langle\langle b[\tilde{\zeta}]\rangle\rangle)=v \circ u(\mathbb{C}\langle\langle b[\tilde{[}]\rangle\rangle b[0])=0$. Therefore $v \circ u=v \circ u \circ \pi$.

Analogues of $G_{0}, G_{1}$ for the equation (64), where $A, b[1]$ are replaced by $A-\alpha, b[1]-\beta$ are $G_{0}(z) z^{\alpha}(1-z)^{\beta}, G_{1}(z) z^{\alpha}(1-z)^{\beta}$, hence the holonomy of this equation is $\Psi$. So $u(\Psi)=\Psi$ (a constant series in $\alpha, \beta)$, so $v \circ u(\Psi)=\Psi$. It follows that

$$
\Psi=v \circ u \circ \pi(\Psi) .
$$

We have

$$
\Psi=\lim _{\varepsilon \rightarrow 0} \varepsilon^{-b[1]}\left(\sum_{\left(\tilde{\zeta}_{1}, \ldots, \tilde{\zeta}_{r}\right) \in\left(\tilde{\mu}_{N}\right)^{r}}(-1)^{\operatorname{card}\left\{i \in[1, r] \mid \tilde{\zeta}_{i} \neq 0\right\}} I_{\varepsilon}\left(\tilde{\zeta}_{1}, \ldots, \tilde{\zeta}_{r}\right) w\left(\tilde{\zeta}_{1}, \ldots, \tilde{\zeta}_{r}\right)\right) \varepsilon^{b[0]}
$$


where $I_{\varepsilon}\left(\tilde{\zeta}_{1}, \ldots, \tilde{\zeta}_{r}\right)$ is defined as $I\left(\tilde{\zeta}_{1}, \ldots, \zeta_{r}\right)$ with integration domain $\varepsilon \leq t_{1} \ldots \leq t_{r} \leq 1-\varepsilon$. Applying $\pi$ to this identity, we get

$$
\pi(\Psi)=\sum_{\left(\tilde{\zeta}_{1}, \ldots, \tilde{\zeta}_{r}\right) \in\left(\tilde{\mu}_{N}\right)^{r} \mid \tilde{\zeta}_{r} \neq 1, \tilde{\zeta}_{1} \neq 0}(-1)^{\operatorname{card}\left\{i \in[1, r] \mid \tilde{\zeta}_{i} \neq 0\right\}} I\left(\tilde{\zeta}_{1}, \ldots, \tilde{\zeta}_{r}\right) w\left(\tilde{\zeta}_{1}, \ldots, \tilde{\zeta}_{r}\right) .
$$

The result now follows from (65), (66) and the fact that $v \circ u\left(w\left(\tilde{\zeta}_{1}, \ldots, \tilde{\zeta}_{r}\right)\right)=\tilde{w}\left(\tilde{\zeta}_{1}, \ldots, \tilde{\zeta}_{r}\right)$.

Remark A.2. Using this proposition, one may use [EG] to give an explicit formula for $\log \left(\Psi_{\mathrm{KZ}}\right)$.

\section{REFERENCES}

[Art] E. Artin, Theory of braids, Ann. of Math., 48 (1947), no. 2, 101-26.

[BN] D. Bar-Natan, On associators and the Grothendieck-Teichmüller group. I, Selecta Math. (N.S.), 4 (1998), no. 2, 183-212.

[Ch] I. Cherednik, Factorizing particles on a half line, and root systems, (Russian), Teoret. Mat. Fiz., 61 (1984), no. 1, 35-44.

[CF] L. Crane, I. Frenkel, Four-dimensional topological quantum field theory, Hopf categories, and the canonical bases, Topology and physics, J. Math. Phys., 35 (1994), no. 10, 5136-5154.

[DG] P. Deligne, A. Goncharov, Groupes fondamentaux motiviques de Tate mixtes, math/0302267, Ann. Sci. Ecole Norm. Sup. (4), 38 (2005), no. 1, 1-56.

[DKM] J. Donin, P. Kulish, A. Mudrov, On a universal solution to the reflection equation, Lett. Math. Phys., 63 (2003), no. 3, 179-94.

[Dr1] V. Drinfeld, Quasi-Hopf algebras, Leningrad Math. J., 1 (1990), 1419-57.

[Dr2] V. Drinfeld, On quasitriangular quasi-Hopf algebras and a group closely connected with $\operatorname{Gal}(\overline{\mathbb{Q}} / \mathbb{Q})$, Leningrad Math. J., 2 (1991), 829-60.

[EE] B. Enriquez, P. Etingof, Quantization of dynamical r-matrices with nonabelian base, preprint math/0311224, Commun. Math. Phys., 254 (2005), no. 3, 603-650.

[EG] B. Enriquez, F. Gavarini, A formula for the logarithm of the KZ associator, math.QA/0404262, SIGMA Symmetry Integrability Geom. Methods Appl. 2 (2006), Paper 080 (electronic) (in memory of V. Kuznetsov).

[EN] P. Etingof, D. Nikshych, Vertex-IRF transformations and quantization of dynamical r-matrices, math.QA/0103079, Math. Res. Letters, 8 (2001), no. 3, 331-346.

[FRT] L. Faddeev, N. Reshetikhin, L. Takhtajan, Quantization of Lie groups and Lie algebras, Leningrad Math. J., 1 (1990), 193-226.

[GL] V. Golubeva, V. Leksin, On two types of representation of the braid group associated with the KnizhnikZamolodchikov equation of the $B_{n}$ type, J. Dynam. Control Systems, 5 (1999), no. 4, 565-596.

[Go] A. Goncharov, Multiple polylogarithms and mixed Tate motives, preprint math/0103059.

[Gr] A. Grothendieck, Esquisse d'un programme, published in Geometric Galois Actions I, P. Lochak and L. Schneps, eds., London Math. Society Lecture Note Series 242, Cambridge University Press (2000).

[Ih1] Y. Ihara, Some arithmetic aspects of Galois actions in the pro-p fundamental group of $\mathbb{P}^{\nVdash}-\{\nvdash, \nVdash, \infty\}$, Arithmetic fundamental groups and noncommutative algebra (Berkeley, CA, 1999), 247-273, Proc. Sympos. Pure Math., 70, Amer. Math. Soc., Providence, RI, 2002.

[Ih2] Y. Ihara, On beta and gamma functions associated with the Grothendieck-Teichmüller group, II, J. Reine Angew. Math., 527 (2000), 1-11.

[Ko] T. Kohno, On the holonomy Lie algebra and the nilpotent completion of the fundamental group of the complement of hypersurfaces, Nagoya Math. J. 92 (1983), 21-37.

[KS] P. Kulish, R. Sasaki, Covariance properties of reflection equation algebras, Prog. Theor. Phys. 89 (1993), no. 3, 741-761.

[LM] T.T.Q. Le, J. Murakami, Kontsevich's integral for the Kauffman polynomial, Nagoya Math. J. 142 (1996), 39-65.

[O] V. Ostrik, Module categories, weak Hopf algebras and modular invariants, Transform. Groups, 8 (2003), no. 3, 177-206.

[Rac] G. Racinet, Doubles mélanges des polylogarithmes multiples aux racines de l'unité, Publ. Math. IHES 95 (2002), 185-231.

[Re] C. Reutenauer, Free Lie algebras, London Mathematical Society Monographs, New Series, 7 (1993), Oxford University Press. 
[Sos] A. Sossinsky, Preparation theorems for isotopy invariants of links in 3-manifolds, LNM 1510 "Quantum Groups" (P. Kulish, ed.), Springer-Verlag.

[Su] D. Sullivan, Infinitesimal computations in topology, Publ. Math. IHES 47 (1977), 260-331.

[V] V. Vershinin, On braid groups in handlebodies, Siberian Math. J., 39 (1998), no. 4, 645-54.

[W] L. Washington, Introduction to cyclotomic fields, second edition, Graduate Texts in Mathematics 83 (1997), Springer-Verlag.

IRMa (CNRS), rue René Descartes, F-67084 Strasbourg, France

E-mail address: enriquez@math.u-strasbg.fr 